\title{
Untersuchung der elektrischen Phasenseparation in dünnen Manganatschichten mit Rastersondenspektroskopie
}

\author{
Dissertation \\ zur Erlangung des Doktorgrades \\ der Mathematisch-Naturwissenschaftlichen Fakultät \\ der Georg-August-Universität zu Göttingen
}

Thomas Becker

Bad Karlshafen 
D 7

Referent: Prof. Dr. K. Samwer

Korreferent: Prof. Dr. K. Bärner

Tag der mündlichen Prüfung: 08.06.2004 
„Im Anfang schuf Gott den Himmel und die Erde.

Und die Erde war wüst und leer, und es lag Finsternis auf der Tiefe, und der Geist Gottes schwebte über den Wassern.

Und Gott sprach: Es werde Licht! Und es ward Licht.

Und Gott sah, dass das Licht gut war, da schied Gott das Licht von der Finsternis.“ Mose 1, 1-4

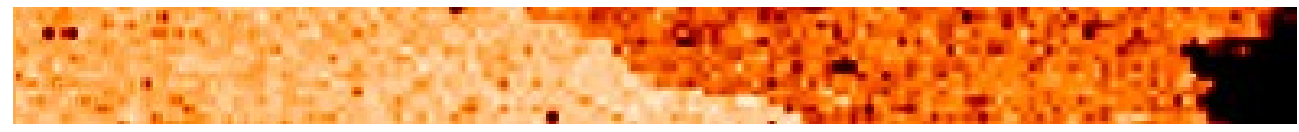




\section{Inhaltsverzeichnis}

1 Einleitung 1

2 Physikalische Grundlagen 4

2.1 Manganate - gemischtvalente Metalloxide 4

$\begin{array}{lll}2.2 & \text { Superaustausch } & 10\end{array}$

$\begin{array}{lll}2.3 & \text { Doppelaustausch } & 11\end{array}$

2.4 Phasenseparation und Perkolationstheorie 14

3 Rastertunnelmikroskopie (STM) und - Spektroskopie (STS) 25

3.1 Theoretische Grundlagen 25

3.2 Die Messapparatur 30

4 Auswerteverfahren $\quad 33$

4.1 Analysemethode der Tunnelspektren 33

4.2 Simulation von Widerstandsnetzwerken 38

5 Ergebnisse und Auswertungen $\quad 44$

$5.1 \quad$ Bestimmung der Austrittsarbeit $\quad 44$

$5.2 \quad$ LCMO auf $\mathrm{MgO}$ bei $78 \mathrm{~K} \quad 46$

$5.3 \quad$ LSMO - Einkristall bei $122 \mathrm{~K} \quad 50$

$5.4 \quad$ LSMO auf $\mathrm{MgO} \quad 53$

$5.5 \quad$ LSMO auf Iridium 59

5.6 Sauerstoffreduziertes LCMO auf Iridium 64

6 Abschließende Diskussion $\quad 73$

7 Zusammenfassung $\quad 88$

8 Anhang 90

8.1 Sauerstoffdegradation 90

8.2 Ergebnisse an Vanadiumoxid 93

8.3 Ergebnisse am $\kappa$-(BETS) ${ }_{2} \mathrm{FeCl}_{4}$ - Einkristall 99

8.4 Wirtschaftliche Analyse und technische Anwendungsgebiete von magnetoresistiven

$\begin{array}{ll}\text { Materialien } & 104\end{array}$

9 Literaturverzeichnis 112

10 Danksagungen 124

11 Lebenslauf $\quad 125$ 


\section{Einleitung}

Den Untersuchungen und dem Verständnis von Oberflächeneigenschaften wird in letzter Zeit immer mehr Bedeutung zugemessen. Das Interesse gilt vor allem magnetischen Systemen, bei denen universitäre und industrielle Forschung erhebliche Anstrengungen unternehmen, um ein Verständnis für elektronische und magnetische Strukturen $\mathrm{zu}$ erlangen. Die in diesem Zusammenhang von großer Bedeutung gekennzeichneten Manganate werden seit der Entdeckung des kolossalen Magnetowiderstandes (CMR) zu Beginn der 90er Jahre [1] für weiterführende Untersuchungen herangezogen.

Manganate zeichnen sich durch eine Perovskitstruktur aus, wobei die Manganionen von einem Sauerstoffoktaeder umgeben sind und einen Jahn-Teller-Effekt aufweisen [2]. Als Ausgangssubstanz kann das elektrisch isolierende und antiferromagnetische $\mathrm{LaMnO}_{3}$ angesehen werden, das durch Dotierung mit z. B. Calcium oder Strontium die elektronischen Eigenschaften wesentlich ändert. Bei geeigneter Dotierungskonzentration stellt sich eine Valenzmischung aus $\mathrm{Mn}^{3+}$ und $\mathrm{Mn}^{4+}$ ein, die einen elektrisch leitfähigen Grundzustand und ferromagnetische Eigenschaften bei tiefen Temperaturen zeigt [3]. Es ist mittlerweile allgemein akzeptiert, dass es sich bei der zugrunde liegenden Wechselwirkung um den Doppelaustauschmechanismus handelt [4]. Diese Wechselwirkung zeigt eine starke Abstands - und Winkelabhängigkeit. Sie ist besonders effektiv, wenn die Mn - O - Mn Bindungen linear ausgerichtet sind, d.h. wenn die Mangan - und Sauerstoffionen in einer Reihe angeordnet vorliegen. Bei Abweichungen des Winkels von $180^{\circ}$ nimmt die elektrische Leitfähigkeit stark ab. Dies kann bis zum isolierenden Verhalten der Jahn Teller - verzerrten Phase und deren Zunahme bei steigender Temperatur führen. In diesem Zusammenhang wird von einem Metall-Isolator-Übergang (MIT ${ }^{1}$ ) gesprochen, dessen temperaturabhängiger Widerstandsverlauf im Rahmen der Perkolationstheorie diskutiert wird.

Die theoretischen Grundlagen des Perkolationsübergangs basieren in diesen Zusammenhang im Wesentlichen auf Überlegungen von Dagotto et al. [5; 6; 7]. Deren Perkolationsszenarien resultieren aus der Grundidee einer chemisch homogenen Probe, die durch elektronische Phasenseparation in metallisch leitfähige und isolierende Domänen zerfällt. Es stellt sich die Frage, warum diese Bereiche bis zu mehreren Nanometern Größe anwachsen können und somit eine Clusterbildung bei Manganaten vorliegt. Hierzu hat Moreo Monte Carlo Simulationen durchgeführt [8]. Als Parameter wurden von ihm die Kopplungsbeziehungen der am Austausch beteiligten Atome, die chemische Zusammensetzung, die zufällige Verteilung von La- und CaIonen und deren unterschiedlichen Ionenradien sowie der Temperatur- und Feldeinfluss

\footnotetext{
${ }^{1}$ Metal Isolator Transition
} 
berücksichtigt. Das Resultat seiner Simulationen ist eine Clusterbildung von antiferromagnetischen $(A F)$ und ferromagnetischen $(F)$ Bereichen und deren Feldabhängigkeit. Mayr erweiterte diese MC-Simulationen um die Temperaturabhängigkeit des Widerstands der leitfähigen und isolierenden Bereiche [6]. Grundgedanke ist die Beschreibung des Kurvenverlaufs von Transportmessungen aus [1] durch ein Ersatzschaltbild von metallisch leitfähigen und halbleitend ,isolierenden“ Widerständen, die parallel verschaltet sind. Im Ergebnis zeigt sich ein vergleichbares Bild zu den Simulationen von Moreo. Durch eine analytische Bestimmung des Gesamtwiderstandes nach Kirkpatrik [9] kann Mayr die bei Transportmessungen gewonnenen Kurvenverläufe reproduzieren. Das Verhältnis der Volumenanteile der verschiedenen Phasen zeichnet sich durch die Einflussnahme von unterschiedlichen Parametern entsprechend der Temperaturabhängigkeit der Domänenbildung oder magnetischen Feldern aus, wodurch eine Variation der Übergangstemperatur möglich ist. Die auftretenden Domänenstrukturen zeigen eine typische Größenverteilung von atomarer bis mesoskopischer Skala und sollen in dieser Arbeit mit Hilfe der Rastersondenmikroskopie untersucht werden (Kapitel 3).

Erst in den letzten Jahren sind Ergebnisse vorgestellt worden, die Erklärungspotential hinsichtlich der Perkolationstheorie besitzen. Messungen von Fäth et al. zeigen, dass rastertunnelspektroskopische Untersuchungen entsprechend genutzt werden können [10]. Die elektronischen Eigenschaften an der Oberfläche eines $\mathrm{LaCaMnO}_{3}$ - Einkristalls sind räumlich inhomogen und besitzen eine Abhängigkeit vom äußeren Magnetfeld. Atomar aufgelöste Messungen von Renner et al. an $\mathrm{Bi}_{0.24} \mathrm{Ca}_{0.76} \mathrm{MnO}_{3}$ - Einkristallen weisen isolierende und leitfähige Strukturen auf, die in direkter Nachbarschaft nebeneinander vorliegen [11].

Ziel dieser Arbeit ist eine weiterführende Analyse der für den Perkolationsübergang verantwortlichen intrinsischen Inhomogenitäten, wobei deren Auftreten ggf. unabhängig von extrinsischen Einflüssen entsprechend Magnetfeldern, Verspannungen oder weiteren äußeren Parametern ist. Bei den untersuchten Materialien handelt es sich um $\mathrm{La}_{\mathrm{x}} \mathrm{Ca}_{1-\mathrm{x}} \mathrm{MnO}_{3}$ (LCMO) und $\mathrm{La}_{\mathrm{x}} \mathrm{Sr}_{\mathrm{x}-1} \mathrm{MnO}_{3}$ (LSMO), die als dünne Filme oder als Einkristall vorliegen. Mit Hilfe rastertunnelspektroskopischer Messmethoden sollen bei unterschiedlichen Temperaturen (25K bis $317 \mathrm{~K})$ Domänenentwicklungen untersucht und zusätzlich elektronische Eigenschaften der Probenoberflächen analysiert werden. Hierbei werden die Unterschiede in der Zustandsdichte ausgenutzt um die verschiedenen Domänenstrukturen zu separieren.

Die so gewonnenen Erkenntnisse über temperaturabhängige, zweidimensionale Leitfähigkeitsverteilungen an der Oberfläche werden in Kapitel 5 zusätzlich mit Transportmessungen verglichen. Ziel ist es, den Übergang zwischen leitendem und nicht leitendem Verhalten (Perkolationsübergang) zu untersuchen und einen Zusammenhang zwischen den Transportmessungen und den STS-Messungen zu erlangen. 
Um diesen Vergleich zwischen Transportmessungen und Leitfähigkeitsmessungen ziehen zu können, werden die Grundlagen der Strompfadsimulation in Kapitel 4 vorgestellt. Diese Methode erlaubt es, den Gesamtwiderstand beliebig großer und rechteckig verschachtelter Widerstandsnetzwerke auf Basis der Kirchhoffschen Gesetze zu bestimmen. Hierbei handelt es sich anders als bei Kirkpatrik [9] um eine rein numerische Methode. Als weiteres Ergebnis der Simulation lassen sich die Stromflussentwicklungen über einen definierten, zweidimensionalen Probenbereich sichtbar machen.

Abschließend sollen in Kapitel 6 die gewonnenen Ergebnisse aus den Rastertunnelmessungen und durchgeführten Magnetisierungsmessungen mit Ergebnissen anderer Gruppen verglichen werden. 


\section{Physikalische Grundlagen}

Die in dieser Arbeit untersuchten Proben $\mathrm{La}_{1-\mathrm{x}} \mathrm{Sr}_{\mathrm{x}} \mathrm{MnO}_{3}$ und $\mathrm{La}_{1-\mathrm{x}} \mathrm{Ca}_{\mathrm{x}} \mathrm{MnO}_{3}$ fallen in die Substanzklasse der dotierten Manganate. Aufgrund ihrer vielfältigen physikalischen Fragestellungen haben sie weltweit für großes Interesse gesorgt. In diesem Kapitel sollen die physikalischen Grundlagen der Manganate dargestellt werden.

Effekte wie der Einfluss des Kristallfeldes auf $\mathrm{MnO}_{6}$ - Oktaeder sowie weitere kollektive Phänomene, die auf die Konkurrenz von Super- und Doppelaustausch führen, sollen erläutert werden. Abschließend folgt eine theoretische Betrachtung, die zur Phasenseparation und zum Perkolationsübergang führen.

\subsection{Manganate-gemischtvalente Metalloxide}

Die Kristallstruktur dotierter Manganate mit der Zusammensetzung $\mathrm{A}_{1-\mathrm{x}} \mathrm{B}_{\mathrm{x}} \mathrm{MnO}_{3}$ entspricht in guter Nährung $\mathrm{CaTiO}_{3}$. Es handelt sich hierbei um das Mineral Perowskit, das nach dem Mineralogen von Perowski benannt wurde. Bei den Manganaten prägen ein Mangan-Ion und ein umgebenden Sauerstoff-Oktaeder diese Struktur (Abbildung 2.1).

Bei den Manganaten befinden sich die Mangan-Ionen an den Ecken einer nahezu kubischen Einheitszelle und sind von je sechs Sauerstoff-Ionen auf den Kanten umgeben. Im Zentrum dieser Zelle befindet sich ein Selten-Erd-Ion (A) oder ein Erdalkali-Ion (B) (Abbildung 2.2).

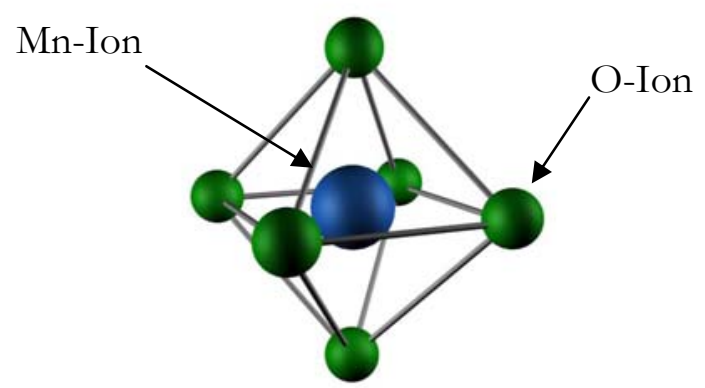

Abbildung 2.1: $\quad$ Mangan-Ion umgeben von dem Sauerstoff-Oktaeder 


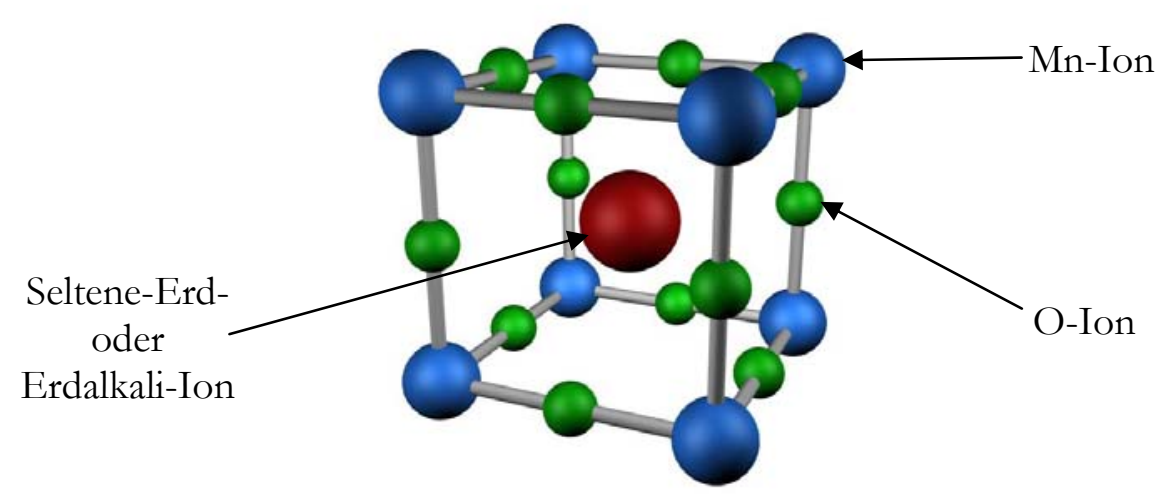

Abbildung 2.2: Kubische Einheitszelle der Manganate

Hierbei wird aufgrund von geometrischen Gründen für die Bindungslängen zwischen dem Gitterplatz des Selten-Erd-Ions und des Sauerstoffs $\left(d_{A, 0}\right)$, bzw. der Position des Mangan-Ions und des Sauerstoffs $\left(d_{B, O}\right)$ ein Verhältnis von $\frac{d_{A, O}}{d_{B, O}}=\sqrt{2}$ eingestellt. Geringe Abweichungen führen zu einer verspannten Struktur, welche sich in dem Toleranzfaktor

$$
T=\frac{1}{\sqrt{2}} \frac{d_{A, O}}{d_{B, O}} \approx \frac{1}{\sqrt{2}} \frac{r_{A}+r_{O}}{r_{B}+r_{O}}
$$

widerspiegeln. Näherungsweise können die Abstände $d_{A, O}$ und $d_{B, O}$ durch die Ionenradien $r_{A}, r_{B}$ und $r_{O}$ ersetzt werden. Für Werte von $T \sim 1$ handelt es sich nach Goldschmidt [12] um Perowskite. Nimmt der Toleranzfaktor ab, ändert sich die Gitterstruktur zunächst in eine rhomboedrische $(0,96<T<1)$ gefolgt von einer orthorombischen Struktur $(T<0,96)$. Laut Goodenough [13] müssen die Positionen der Ionen auf den A- und B- Plätzen innerhalb einer sechs- bzw. zwölfstelligen Koordination stabil sein, wodurch sich die Radien auf $r_{A}>0,90 A$ und $r_{B}>0,51 A$ beschränken. Eine Inkompatibilität der ionischen Radien kann zur Verdrehung des Sauerstoffoktaeders führen. Diese neuen Kristallstrukturen können eine niedrigere Symmetrie besitzen, weiterführende Informationen dazu sind bei Coey [14] zu finden. Betrachtet man undotiertes $\mathrm{LaMnO}_{3}$, erkennt man, dass die Sauerstoffionen zweifach negativ geladen sind $\left(\mathrm{O}^{2-}\right)$ und das Lanthan als dreiwertiges $\mathrm{La}^{3+}$ vorliegt. Dementsprechend liegen die Manganionen als dreifach positiv geladen vor $\left(\mathrm{Mn}^{3+}\right)$ und besitzen die Elektronenkonfiguration $3 \mathrm{~d}^{4}$. Es existieren fünf mögliche d-Orbitale (Abbildung 2.3). Da sich Manganionen in direkter Nachbarschaft zu den Sauerstoffionen befinden, sind die $e_{g}$-Orbitale energetisch ungünstiger als die $t_{2 g}$-Orbitale. $e_{g}$ Orbitale besitzen eine höhere Aufenthaltswahrscheinlichkeit der Elektronen in Richtung des Sauerstoffs und somit eine höhere Coulombabstoßung. $t_{2 g}$-Orbitale liegen genau zwischen den 
Achsen und sind daher energetisch günstiger. Deshalb belegen die vier d-Elektronen des $\mathrm{Mn}^{3+}$ Ions die drei $t_{2 g}$-Orbitale und ein $e_{g}$-Orbital. Aufgrund der Hundschen Regel sind die Spins parallel ausgerichtet. Da zwei energetisch gleichwertige $\mathrm{e}_{\mathrm{g}}$-Orbitale vorliegen, ergeben sich im Idealfall einer kubischen Symmetrie für das Gesamtsystem zwei energetisch entartete Zustände (das Elektron kann das $3 d_{z^{2}}$ - Orbital oder das $3 d_{x^{2}-y^{2}}$ - Orbital besetzen).
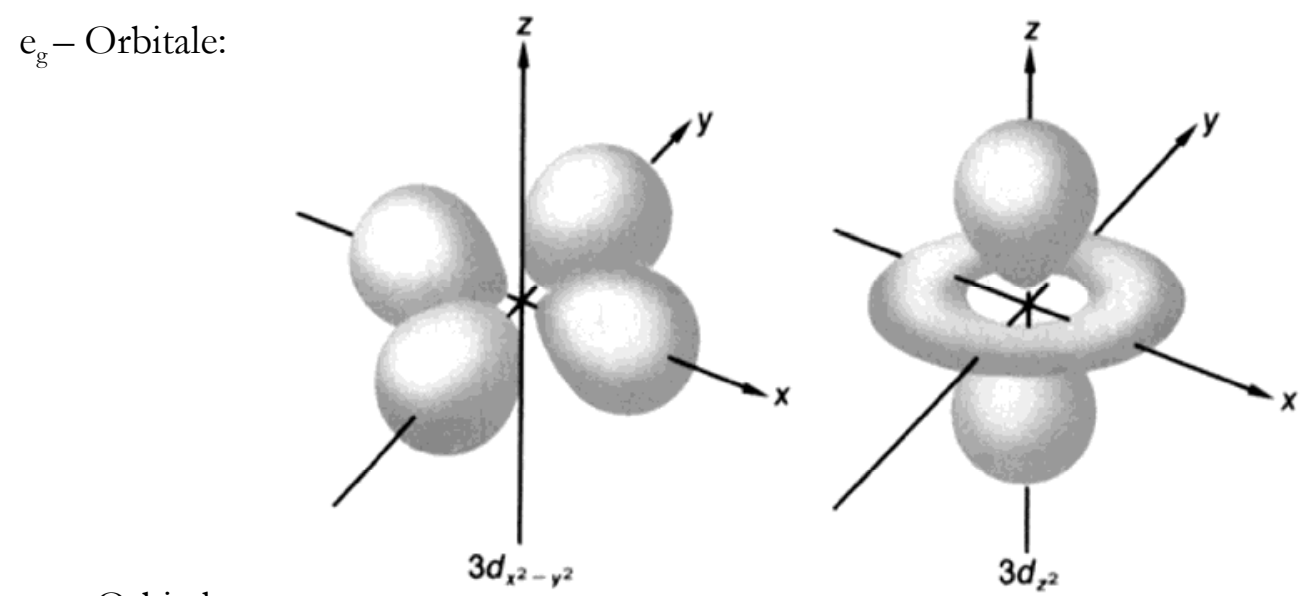

$t_{2 g}$ - Orbitale:
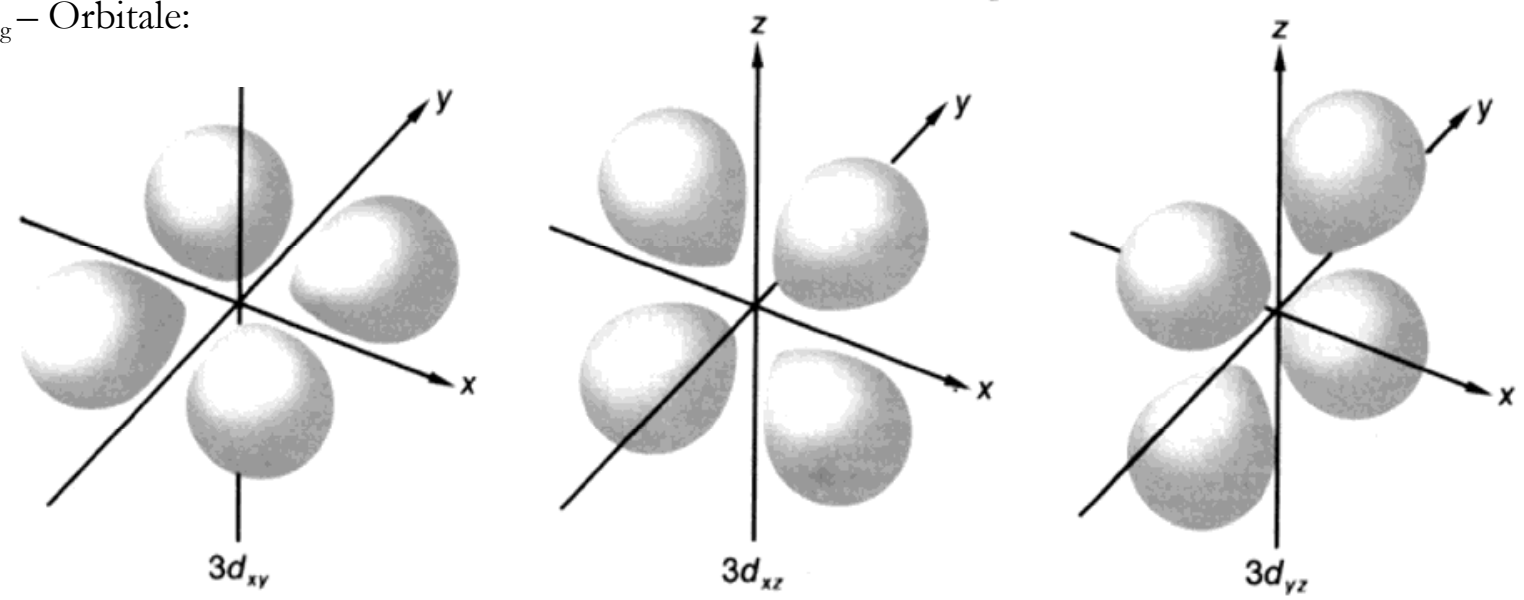

Abbildung 2.3: Darstellung der d-Orbitale (aus Mortimer [15])

Da zwei entartete Zustände vorliegen, hat das System noch keine Potentialminimierung zur Senkung seiner Energie durchgeführt. Durch die so genannte Jahn - Teller - Verzerrung [2] erniedrigt das Gesamtsystem zusätzlich seine Energie, was zu einer energetischen Aufsplittung der $e_{g}-$ und $t_{2 g}$-Orbitale führt. Eine mögliche Verzerrung besteht in einer Streckung des Sauerstofftetraeders in $\mathrm{z}$-Richtung und einer gleichzeitigen Kompression in $\mathrm{x}$ - und $\mathrm{y}$-Richtung (siehe Abbildung 2.4). 

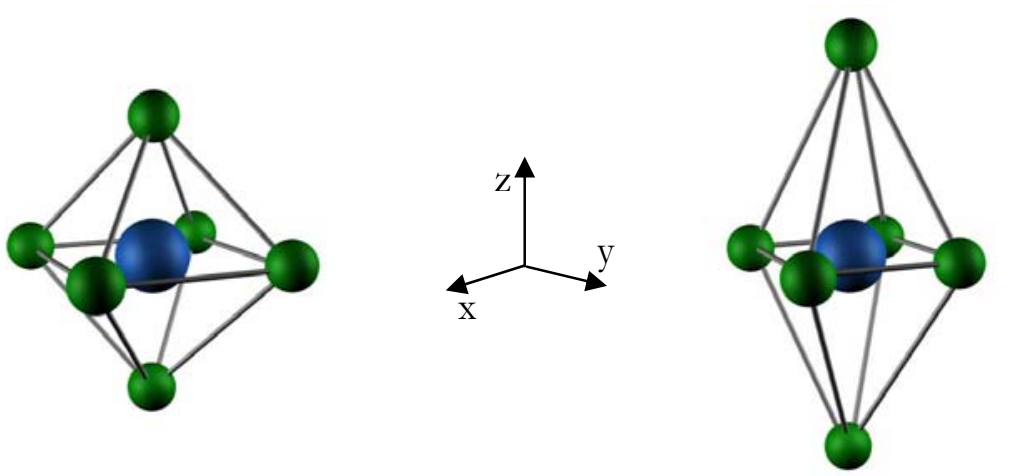

Abbildung 2.4: Skizzierte Verzerrung des Sauerstoffoktaeders infolge des Jahn-Teller-Effekts (die Deformation ist zur Veranschaulichung stark überzeichnet)

Durch die Verzerrung entlang der z-Achse wird das $3 d_{z^{2}}$ - Orbital, das seine größte Ausdehnung in z-Richtung hat, aufgrund der sinkenden Coulombabstoßung energetisch begünstigt. Die Stauchung in der $x-y$-Ebene erhöht indes die Energie, da das $3 d_{x^{2}-y^{2}}$ - Orbital in $x-y-R i c h t u n g$ die größte Aufenthaltswahrscheinlichkeit hat. Die Aufspaltung der Energieniveaus der $3 d$-Orbitale ist in Abbildung 2.5b schematisch dargestellt. Der Gewinn an elektrostatischer Energie steigt linear mit der Verzerrung an, während der Energieverlust durch rücktreibende, elastische Kräfte wächst.

Bei $\mathrm{LaMnO}_{3}$ liegt eine elektronische Struktur gemäß Abbildung 2.5b vor. Das vierte Elektron des $\mathrm{Mn}^{3+}$ - Ions besetzt in diesem Fall das energetisch niedrigere $3 d_{z^{2}}$ - Orbital. Im Kristall würde diese lokale Strukturverzerrung zu starken Verspannungen führen. Diese werden aber durch eine geeignete Anordnung (drehen und kippen) der einzelnen Oktaeder stark kompensiert (Abbildung 2.6 (a) und (b)). Die x- und y-Achsen werden dadurch vergrößert und die z-Achse gestaucht. Man spricht hier von einem kooperativen statischen Jahn-Teller-Effekt mit einer permanenten Verzerrung des Gitters.

a)

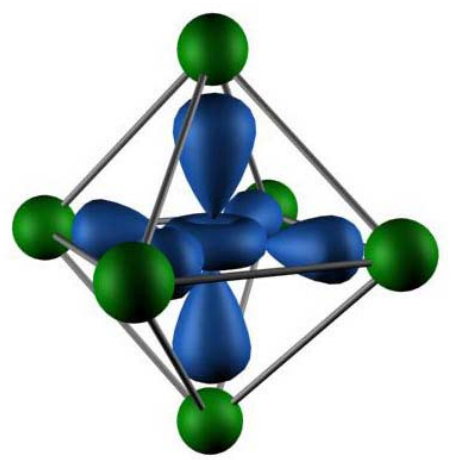

b)

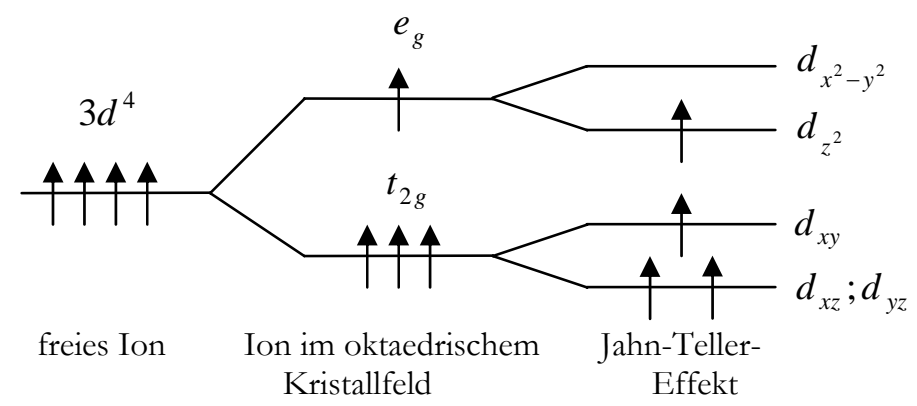

Abbildung 2.5: Aufspaltung der Energieniveaus der 3d-Orbitale durch das Kristallfeld und durch den Jahn-Teller-Effekt (schematisch) [16] 
Die magnetischen Dipol-Dipol-Wechselwirkungen zwischen Spins benachbarter Mangan-Ionen sind vergleichsweise schwach. Dies führt unter anderem dazu, dass bei $\mathrm{LaMnO}_{3}$ die einzelnen Ebenen ferromagnetisch geordnet sind, aber mit entgegengesetzter Magnetisierungsrichtung antiferromagnetisch gestapelt vorliegen (Abbildung 2.6 (c)) [17; 18].
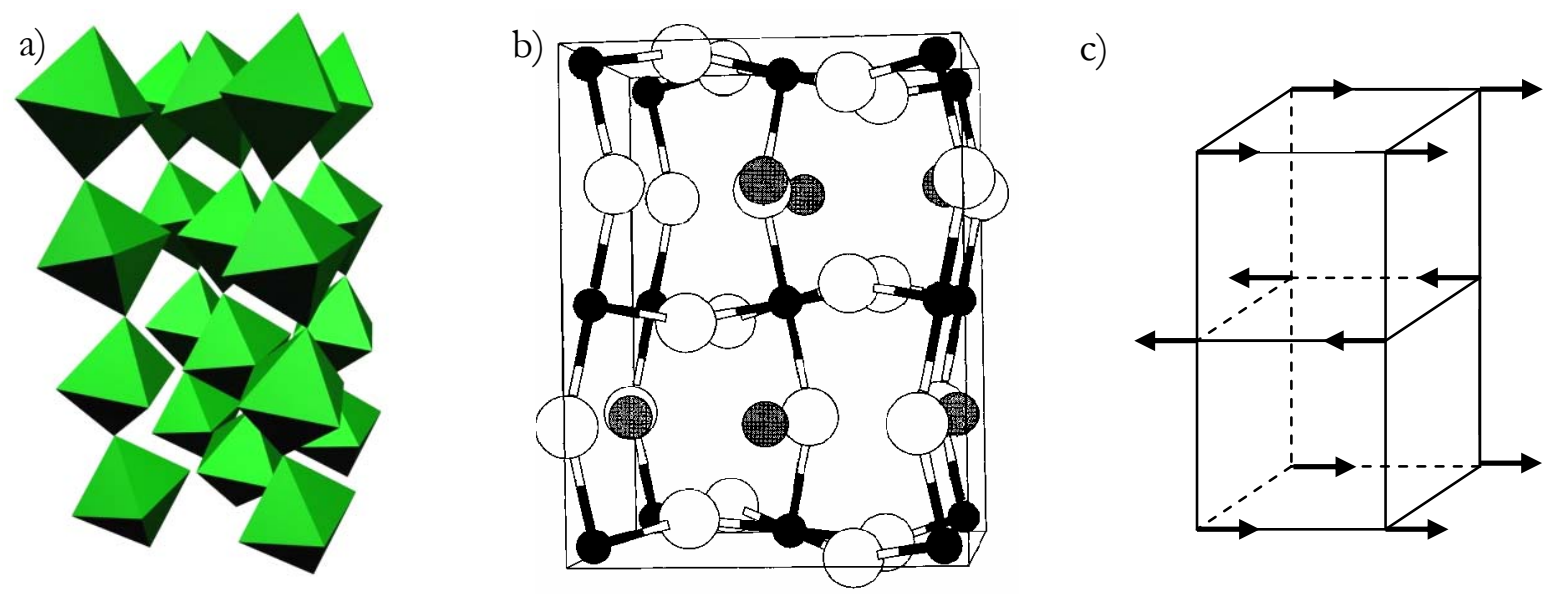

Abbildung 2.6: (a) und (b) gedrehte und verkippte Anordnung der Oktaeder um den Jahn-Teller-Effekt auszugleichen[19]; (c) antiferromagnetische Ordnung von $\mathrm{LaMnO}_{3}$

Betrachtet man andererseits reines $\mathrm{SrMnO}_{3}$ ordnet auch dieses antiferromagnetisch. Strontium ist zweiwertig und liegt im „Edelgaszustand“ $\left(\mathrm{Sr}^{2+}\right)$ vor. Dadurch liegen nun $\mathrm{Mn}^{4+}$ - Ionen vor, wodurch das $\mathrm{e}_{\mathrm{g}}$ - Niveau unbesetzt ist: Es tritt keine Jahn-Teller-Verzerrung auf.

Ersetzt man im $\mathrm{LaMnO}_{3}$ zunehmend $\mathrm{Mn}^{3+}$ durch $\mathrm{Mn}^{4+}$, so fügt man dem System auf einzelnen Gitterplätzen Löcher zu. An diesen Plätzen tritt somit keine Jahn-Teller-Verzerrung auf, während die Nachbarschaft davon betroffen ist. Dieses Loch hat jedoch die Möglichkeit, auf benachbarte Gitterplätze zu hüpfen, d.h. die Lokalisation von $\mathrm{Mn}^{4+}$ und $\mathrm{Mn}^{3+}$ fluktuiert zeitlich und räumlich. Die lokale strukturelle Verzerrung wandert dabei mit. Diese Wechselwirkung bezeichnet man als Polaron [3].

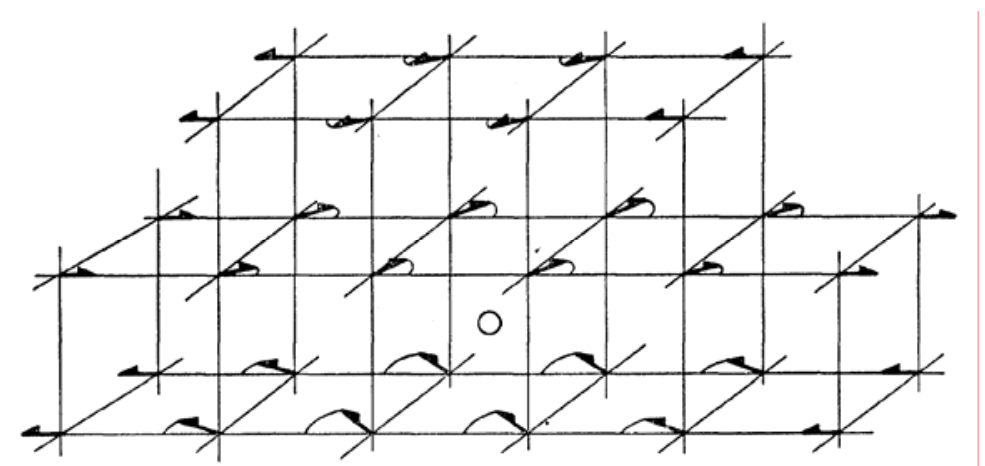

Abbildung 2.7: lokale Spinänderung in $\mathrm{LaMnO}_{3}$ durch Implementierung eines Lochs (z.B. durch Dotierung mit Strontium oder Calcium) 
Der zuvor beschriebene statische Jahn-Teller-Effekt wird so zu einem zeitlich veränderlichen Effekt, man spricht von dem dynamischen Jahn-Teller-Effekt. Die Implementierung eines Loches hat laut de Gennes [20] eine lokale Spinänderung zur Folge. Das anfangs antiferromagnetisch geordnete Spinsystem (Abbildung 2.6 (c)) wird nahe dem Loch umgeordnet (siehe Abbildung 2.7) und bildet die Grundlage für den „Doppelaustausch“. Bandstrukturrechnungen [19; 21;22] und spektroskopische Experimente [23; 24; 25] zeigen, dass dieses Bild von $\mathrm{Mn}_{d 3}$-Leitungsbändern der Realität recht nahe kommt. Aus dem Wechselspiel von Gitterverzerrungen, magnetischen Wechselwirkungen und Polaronen folgen reichhaltige Phasendiagramme mit einer Vielzahl von Phasen (Abbildung 2.8).
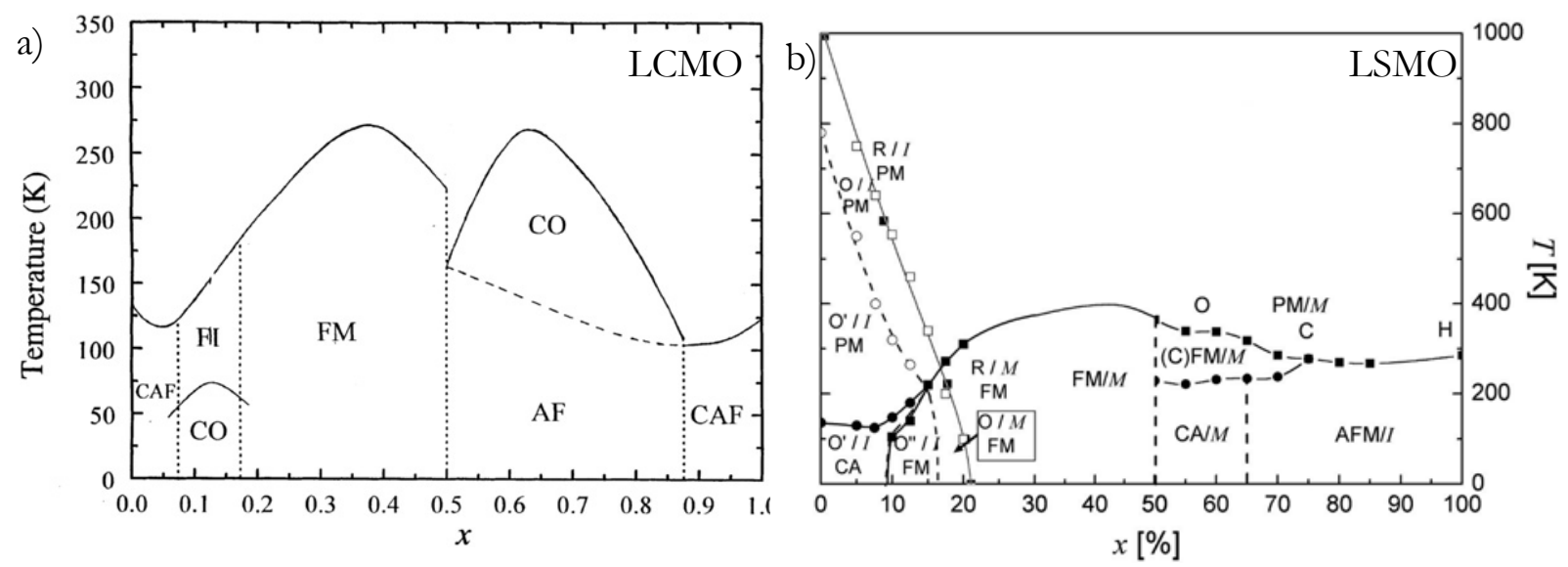

Abbildung 2.8: Phasendiagramme von (a) $\mathrm{La}_{1-\mathrm{x}} \mathrm{Ca}_{\mathrm{x}} \mathrm{MnO}_{3}[3 ; 26 ; 27]$ und (b) $\mathrm{La}_{1-\mathrm{x}} \mathrm{Sr}_{\mathrm{x}} \mathrm{MnO}_{3}$ [28; 29; 30]. Die Bezeichnungen für (a) sind: FM: ferromagnetisches Metall, $C O$ : Isolator mit Ladungsordnung, $A F$ : antiferromagnetischer Isolator, $C A F$ : Isolator mit gekanteter Spinstruktur

Entsprechend für (b): PM: paramagnetisch, $C A$ : gekantet, $A F M$ : antiferromagnetisch, FM: ferromagnetisch, $I$ bzw. $M$ gibt an, ob ein Isolator oder Metall vorliegt 


\subsection{Superaustausch}

Generell unterscheidet man direkte und indirekte Austauschwechselwirkungen. Bei der direkten Wechselwirkung handelt es sich um eine Kopplung in Systemen, deren magnetisches Moment von denselben Elektronen herrührt wie die eigentliche Kopplung. Typisch für diese Form sind Übergangsmetalle, bei denen die $3 d$ - Zustände sowohl das magnetische Moment erzeugen als auch mit den Nachbaratomen überlappen.

a)

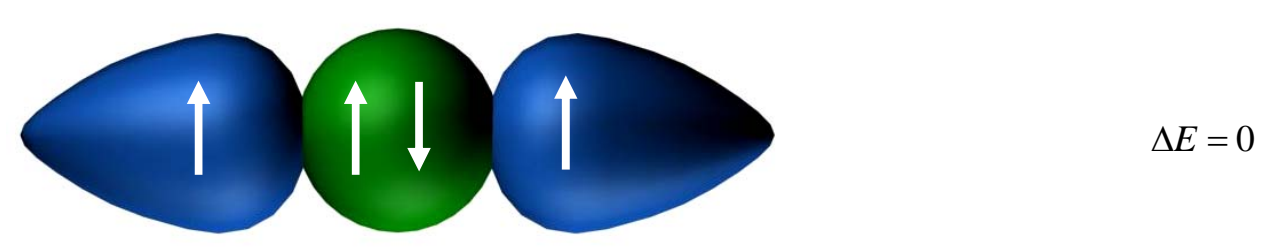

b)

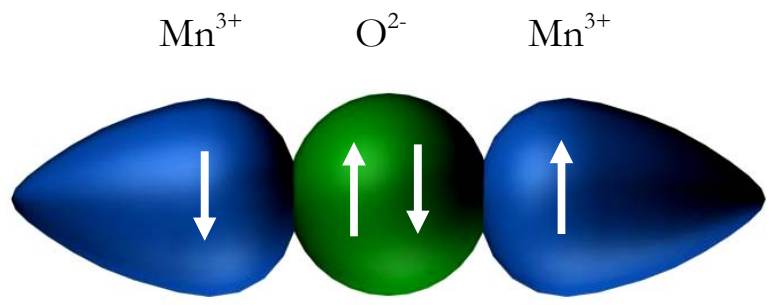

$$
\Delta E=-\frac{2 t^{2}}{U}
$$

Abbildung 2.9: a) gleicher Spin bei den Mn-Ionen (ferromagnetisch)

b) ungleicher Spin bei den Mn-Ionen führt entsprechend dem Hubbard-Modell zu einer Energieerniedrigung (antiferromagnetisch) [31; 32]

Der Austauschmechanismus bei undotiertem $\mathrm{LaMnO}_{3}$ basiert auf einer indirekten Spin-SpinWechselwirkung. Der Abstand zwischen den für den Austausch relevanten Mangan-Ionen ist mit ca. $4 \AA \mathrm{zu}$ groß, um eine direkte Überlappung der $d$-Wellenfunktionen darzustellen. Somit können direkte Austauschmechanismen nicht greifen. Durch das unmagnetische Sauerstoff-Ion zwischen den beiden Manganionen ist es jedoch möglich, einen indirekten Austausch ohne Ladungstransfer zu erhalten. Dies geschieht durch die Überlappung der $3 d$-Orbitale des Mangans und der 2p-Orbitale des Sauerstoffs. Energetisch tiefer liegende Orbitale tragen nichts zum Austausch bei. Aufgrund des relativ großen Abstandes bezeichnet man diese Form als „Superaustausch“. Das p-Orbital des Sauerstoffs ist mit zwei Elektronen gefüllt. Im Mangan befinden sich drei Elektronen in den $t_{2 g}$-Orbitalen (sie bleiben stets am Mangan) und ein Elektron im durch den Jahn-Teller-Effekt energetisch günstigeren $e_{g}$-Orbital. In Abbildung 2.9 sind zwei der möglichen Konfigurationen dargestellt. Somit ist ersichtlich, dass die Kopplung sowohl eine ferromagnetische wie auch eine antiferromagnetische Ordnung zur Folge haben kann. Generell 
wird das System versuchen seine Energie zu minimieren - es wird sich antiferromagnetisch ordnen. Weiterführende Literatur findet man unter [31] und [32].

\subsection{Doppelaustausch}

Das undotierte System $\mathrm{LaMnO}_{3}$ besitzt eine antiferromagnetische Ordnung. Die Manganionen haben dort immer die gleiche Wertigkeit $\mathrm{Mn}^{3+}$. Dotiert man dieses System z.B. mit Calcium oder Strontium entstehen freie Ladungsträger, die zu einer ferromagnetischen Kopplung der MnIonen führen können. Im $\mathrm{La}_{1-\mathrm{x}} \mathrm{Sr}_{\mathrm{x}} \mathrm{MnO}_{3}$ haben Jonker und van Santen [33, 34; 35] im Dotierungsbereich von etwa $x=20 \%$ bis $x=50 \%$ bei Raumtemperatur Ferromagnetismus beobachtet. Dabei wurde ein Zusammenhang zwischen ferromagnetischer Curie-Temperatur und dem elektrischen Widerstand festgestellt.

Dotiert man $\mathrm{LaMnO}_{3}$ mit Strontium liegen im Kristall sowohl $\mathrm{Mn}^{3+}$-Ionen mit vier $d$-Elektronen als auch $\mathrm{Mn}^{4+}$-Ionen mit drei d-Elektronen in der äußersten Schale vor. $\mathrm{Mn}^{3+}$ besitzt im Gegensatz zum $\mathrm{Mn}^{4+}$ ein $3 d_{z^{2}}$-Elektron, das zum Ladungstransport dienen kann. Zener [36] stellte dazu ein Modell des Doppelaustausches vor. Dieses Elektron wechselt vom $\mathrm{Mn}^{3+}$ zum Sauerstoff - simultan wird ein weiteres Elektron vom Sauerstoff an das $\mathrm{Mn}^{4+}$ abgegeben. Das Elektron ist somit gewandert ohne dass eine Anregungsenergie $U$ in Höhe der Coulombabstoßung aufgebracht werden musste - es gibt keine Doppelbesetzung. Das Ergebnis ist eine dem Ausgangszustand energetisch entartete Konfiguration. Diesem „Doppelhüpfprozess“ verdankt der Doppelaustausch seinen Namen.

Goodenough [4] hat eine recht anschauliche Darstellung zum elektrischen Widerstand und zur magnetischen und kristallografischen Ordnung an Manganaten vorgestellt. Er beschreibt den Austauschmechanismus über kovalente und semikovalente Bindungen zwischen dem Sauerstoffund Manganion und macht die Annahme, dass $\mathrm{Mn}^{3+}$-Ionen $d s p^{2}$ (koplanar) und $\mathrm{Mn}^{4+}$-Ionen $d^{2} s p^{3}$ (oktaedrisch) hybridisiert sind. Die magnetische Wechselwirkung der Manganionen koppelt über das $\mathrm{O}^{2-}$ Anion. Hieraus ergeben sich vier verschiedene Bindungstypen, die in Abbildung 2.10 dargestellt sind. Typ 4 zeigt aufgrund seiner gemischtvalenten Manganionen ein dem Doppelaustausch vergleichbares Verhalten. Goodenoughs Aussagen über reines $\mathrm{LaMnO}_{3}$ wurden von Wollan und Köhler [37] bestätigt. 


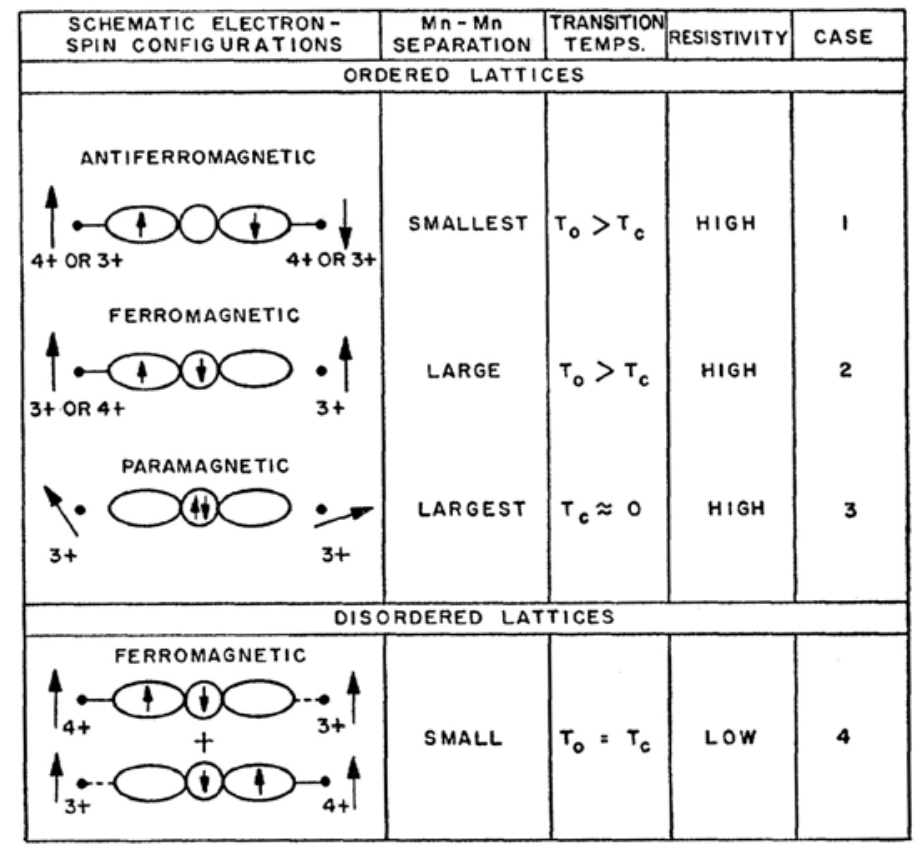

Abbildung 2.10: Schematische Spinkonfiguration nach Goodenough [4]; Typ 1: kovalente Bindung; Typ 2: semikovalente Bindung; Typ 3: paramagnetische Kopplung und Typ 4: ferromagnetisch durch Doppelaustausch

Die bisher vorgestellten Modelle sind leider aus rein theoretischer Sicht unbefriedigend, beschreiben sie doch nur empirisch und stark vereinfacht den Doppelaustausch. Anderson und Hasegawa [38] wählten zur Darstellung des Doppelaustauschmodells eine quantenmechanische Beschreibung. Ausgangspunkt ist die Darstellung der beiden Manganrumpf - Spins einer Mn O - Mn - „Kette“ als Vektoren $\vec{S}_{1}$ und $\vec{S}_{2}$. Beide schließen zueinander einen Winkel $\theta$ ein und besitzen die Spinquantenzahl $S$. Weiterhin wird angenommen, dass noch ein Elektron existiert, welches sich an einem der beiden Rumpfionen befindet und durch Hüpfprozesse den Platz wechseln kann. Es besitzt den Spin $\vec{s}$ (Abbildung 2.11), der aufgrund der starken Hundschen Kopplung $(1,5 \mathrm{eV})$ parallel zu dem Rumpfspin steht. Das interatomare Austauschintegral $J$ und das Elektron-Transfer-Integral $b$ bestimmen die Energie des Systems. Für die Energieeigenwerte des Elektronentransportes gilt näherungsweise:

$$
E=-J S \pm b \cos \theta / 2
$$

Im positiven Fall $(+b \cos \theta / 2)$ liegt eine antiparallele Ausrichtung des Elektrons zum Rumpfspin vor. Der negative Zustand $(-b \cos \theta / 2)$ ist bei paralleler Ausrichtung vorhanden - der Ladungstransport wird begünstigt. Es ist auch eine starke Winkelabhängigkeit der Bindung zu 
erkennen: Je größer der Winkel $\theta$ wird, desto unwahrscheinlicher ist der Ladungstransport. De Gennes [20] hat unter Zuhilfenahme der Winkelabhängigkeit Antiferro-, Ferro- und Paramagnetismus in Abhängigkeit von Temperatur und Dotierung erklärt. Recht anschauliche Darstellungen und Rechnungen zum quantenmechanischen Doppelaustauschmodell findet man unter [39; 40; 41].

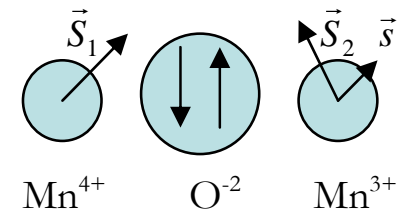

Abbildung 2.11: Vektormodell des quantenmechanischen Doppelaustauschs: $\vec{S}_{1}$ und $\vec{S}_{2}$ kennzeichnen die Rumpfspins der Mn-Ionen, $\vec{s}$ den Spin des freien Ladungsträgers

Es ist jedoch zu beachten, dass auch in diesem Modell Vereinfachungen vorgenommen wurden. Gitterverzerrungen, die z.B. durch den Jahn-Teller-Effekt auftreten und somit Bindungswinkel beeinflussen können, werden ebenso wenig berücksichtigt, wie die unterschiedlichen Coulombwechselwirkungen der Manganionen. Ein $\mathrm{Mn}^{4+}$ Ion zieht benachbarte Sauerstoffionen stärker an als in $\mathrm{Mn}^{3+}$ Ion, was zu einer Änderung der Orbitalüberlappungen führt. Als Konsequenz kann man nicht mehr von energetisch gleichen $d$-Orbitalen und einer Entartung von Anfangs- und Endzustände sprechen. Experimentelle Ergebnisse von Millis [42; 43] unterstreichen diese Unstimmigkeit, indem die Diskrepanz des experimentell bestimmten temperaturabhängigen Widerstandsverlauf und $\mathrm{T}_{\mathrm{C}} \mathrm{zu}$ den theoretisch vorausgesagten Werten aufzeigt werden. Diese Unterschiede sollen unter anderem durch die Existenz von Polaronen ${ }^{2}$ erklärt werden. Millis [43] argumentiert, dass zusätzlich noch ein dynamischer Jahn-Teller-Effekt vorliegt, bei dem aber nur sehr kleine Polaronen gebildet werden, diese aber die Diskrepanzen besonders oberhalb des Übergangs verringern.

\footnotetext{
2 Unter einem Polaron versteht man die Wechselwirkung einer Ladung und der daraus resultierenden lokale Gitterverzerrung. Es handelt sich somit um eine Elektron-Phonon-Wechselwirkung.
} 


\subsection{Phasenseparation und Perkolationstheorie}

Super- und Doppelaustausch verfügen über großes Erklärungspotential hinsichtlich der Leitungsmechanismen in Manganaten; sie alleine reichen aber nicht aus, um diese zu beschreiben. Der von Millis beschriebene Ladungstransport über Polaronen zeigt, dass Erweiterungen von Nöten sind, um ein besseres Verständnis über das System zu erlangen. Erst die mesoskopischen Untersuchung und Beschreibung des Manganatsystems führt auf eine Phasenseparation und zu Perkolationseffekten, die das makroskopische Widerstandsverhalten auf mikroskopische Einflüsse zurückführt.

Dagotto [44] versucht diese Diskrepanz zu lösen, indem er rein theoretische Ansätze verwendet und einen Vergleich zwischen theoretischen Simulationen und realen Messungen anstrebt. Er bezeichnet den reinen Einfluss des Doppelaustausches als „Ein - Orbitalmodell“ und die Erweiterung um Jahn-Teller-Phononen als „Zwei - Orbitalmodell“. Im ersten Fall wird davon ausgegangen, dass die Kopplung der lokalisierten Rumpfspins vernachlässigt wird. Somit ergeben sich die folgenden möglichen Ordnungen [45]: Antiferromagnetismus bei einer Dichte der mobilen Elektronen entsprechend $\left\langle_{n}\right\rangle=1$, Ferromagenetismus bei einer mittleren Elektronenbzw. Lochdichte und ein Phasengemisch bestehend aus den beiden magnetischen Ordnungen.
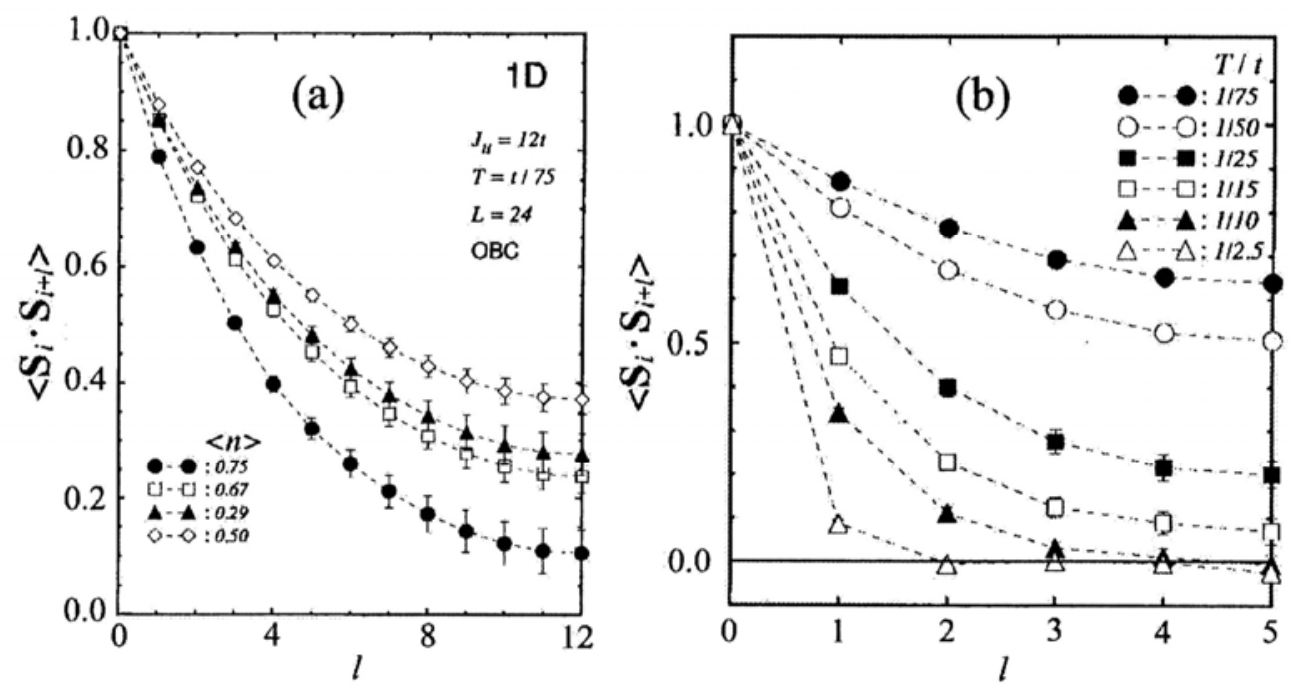

Abbildung 2.12: Abhängigkeit der Spinkorrelation $\left\langle S_{i} S_{i+l}>\right.$ als Funktion der Korrelationslänge 1 von der Dichte der mobilen Elektronen (a) und von der vorgegebenen Temperatur (b) errechnet unter Zuhilfenahme von Monte Carlo Simulationen [44]. Bei mittlerer Elektronen - Loch Konzentration bzw. bei tiefen Temperaturen ist die Kopplung am stärksten und reicht somit am weitesten. 
Im Fall des Ferromagnetismus wird die Spin-Spin Wechselwirkung zweier benachbarter Gitterpunkte betrachtet - liegen beide Spins perfekt parallel zueinander, ist die Spin Korrelation maximal. Ferromagnetismus kann dann vorliegen, wenn diese Kopplung eine lange Reichweite besitzt und sich über mehrere Gitterplätze hinweg erstreckt. Sowohl die Dichte der mobilen Elektronen $<\mathrm{n}>$ als auch die Temperatur haben Einfluss auf die Korrelationslänge (Abbildung 2.12). Yonoki [45] hat den Einfluss von $T_{C}$ auf die Simulationen untersucht. Unterhalb dieser kritischen Temperatur wachsen die Korrelationslängen rasch an. Abbildung 2.13 (a) zeigt eine symmetrische Verteilung um $<\mathrm{n}>=0.5$, die nach beiden Seiten abfällt.

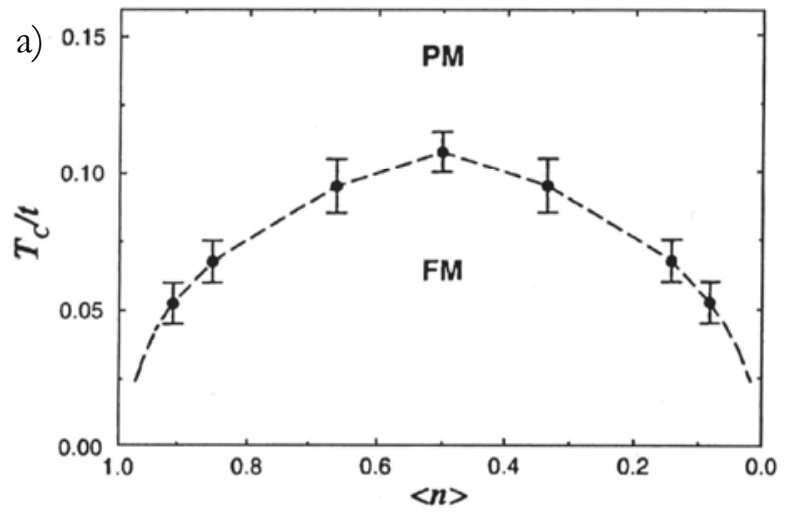

b)

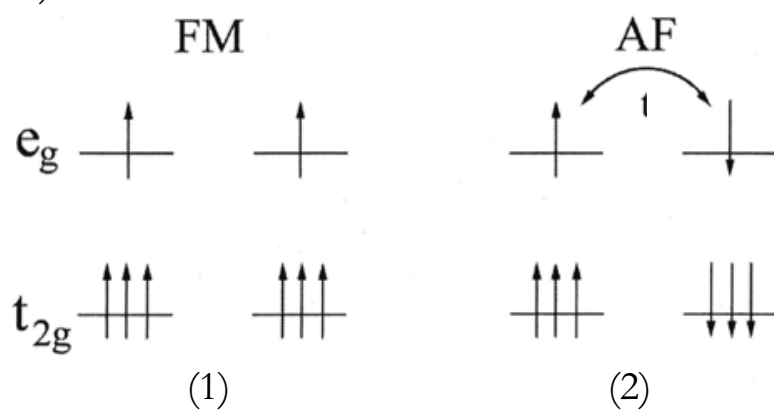

Abbildung 2.13: a) Einfluss der kritischen Temperatur $\mathbf{T}_{\mathrm{C}}$ und der Dichte der mobilen Elektronen auf das magnetische Verhalten. Das Diagramm ist symmetrisch um $<\mathrm{n}>=0.5$ und fällt an den Rändern stark ab.

b) Ferromagnetische Kopplung (1) bei starker Hundscher Wechselwirkung und $<$ n $>=1$ führt aufgrund des Pauliprinzips zu einem Einfrieren der $e_{g}$ - Elektronen. Bildet sich ein antiferromagnetischer Grundzustand aus (2), wird die Energie des Systems erniedrigt und die $e_{g}-$ können sich bewegen. Dementsprechend wird nur der energetisch günstigere, zweite Zustand vorliegen.

Bei den Randelektronendichten ist antiferromagnetisches Verhalten anzutreffen. Liegt ein leeres $e_{g}-$ Band vor $(<\mathrm{n}>=0)$ gibt es keine mobilen Ladungsträger. Daher existiert als einzige, geringe Kopplung die der lokalisierten Spins. Aufgrund der Energieerniedrigung des Systems ist sie antiferromagnetisch. Auf der anderen Seite $(<\mathrm{n}>=1)$ besitzt jedes $e_{g}-$ Niveau ein Elektron das aufgrund der Hund'schen Wechselwirkung parallel zum $t_{2 g}$ - Rumpfspin ausgerichtet ist. Im Falle einer gleichgerichteten, parallelen Ausrichtung der $t_{2 g}$ - Spins zweier benachbarter Atome würde Ferromagnetismus entstehen, die $e_{g}$ - Elektronen wären aber aufgrund des Pauliprinzips nicht mehr beweglich (Abbildung 2.13 (b1)). Sobald die $t_{2 g}$ - Elektronen aber antiferromagnetisch koppeln, können die Elektronen des $e_{g}$ - Niveaus zu Nachbarplätzen springen und dabei nicht das Pauli-Prinzip verletzen - die Energie des Systems wird erniedrigt (Abbildung 2.13 (b2)). 
Die bisherigen Betrachtungen zeigen, dass Ferromagnetismus bei mittlerer Elektronendichte (0.5) bevorzugt wird, während ein antiferromagnetischer Grundzustand bei $<\mathrm{n}>=1$ und 0 vorliegt. Nun stellt sich die Frage, wie sich beide Zustände verhalten, wenn man die Dichte von 1 nach 0.5 variiert. Wie verhalten sich die kurzreichweitigen Wechselwirkungen, wenn eine Phase in die andere übergeht? Der Wechsel beider Zustände muss hierbei als ein Phasenübergang erster Ordnung vonstatten gehen und wird durch das chemische Potential $\mu$ als externer Parameter eingeführt. Dagotto zeigt, dass eine kontinuierliche Variation von $\mu$ eine sprunghafte Änderung der Elektronendichte $\rho$ zur Folge haben kann und somit einige Dichten als unstabil bezeichnet werden müssen. Gibt man ein chemisches Potential $\mu$ und eine solche Dichte $\rho$ vor, zerfällt das anfänglich homogene System in ferromagnetische und antiferromagnetische Bereiche mit Ausmaßen von einigen Nanometern. Dieses Phänomen führt zu einer makroskopischen Phasenseparation, wie sie z.B. bei Supraleitern vom Typ II gefunden wird [46]. Man bezeichnet sie bei Manganaten auch als elektronisch, da die Elektronenverteilungen Einfluss auf die Phasenbildung haben. Lange bevor dieser Begriff bei Supraleitern und Manganaten populär wurde, hatte Nagaev [47] die ersten theoretischen Grundlagen geschaffen und die Phasenseparation zwischen ferro- und antiferromagnetischen Bereichen diskutiert.

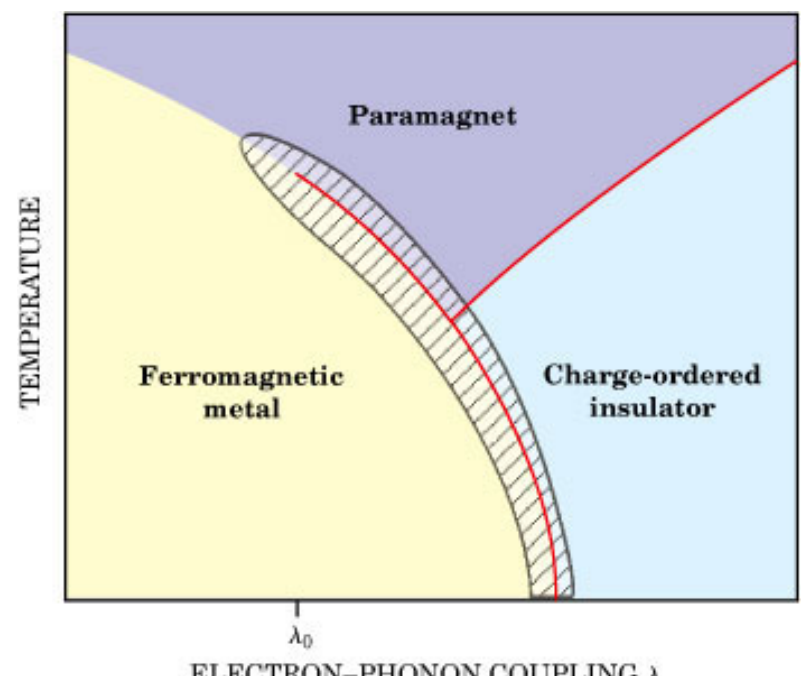

Abbildung 2.14: Allgemeines Phasendiagramm für Manganate [48]. Bei schwacher Elektron-Phonon Kopplung (links) bilden sich ferromagnetische bzw. paramagnetische Phasen aus. Hohe Kopplungen führen zu einer Lokalisierung der Ladungsträger, es entsteht der so genannte „Charge-ordered insulator“. Die roten Linien stellen Phasenübergänge erster Ordnung dar - in ihrer Nähe kann es zur Phasenseparation kommen. Der „Colossal magnetoresistance effect“ liegt im gestreiften Bereich vor (nähere Informationen zum CMR sind bei $[1 ; 33 ; 49]$ zu finden) 
Die Erweiterung des oben vorgestellten Modells um klassische Jahn-Teller-Phononen bildet die Grundlage des „zwei Orbital Modells“. Als neuer, wichtiger Faktor wird hier die ElektronPhonon-Kopplung $\lambda$ eingeführt. Sie beschreibt die Stärke, mit welcher die Valenzelektronen mit dem Kristallgitter wechselwirken. Beispielsweise versucht ein System seine Energie zu minimieren und bildet viele Jahn-Teller-Oktaeder aus. Dies führt zu einer großen Kopplung $\lambda$ und begünstigt somit eine Ladungsaufteilung (siehe Abbildung 2.14).
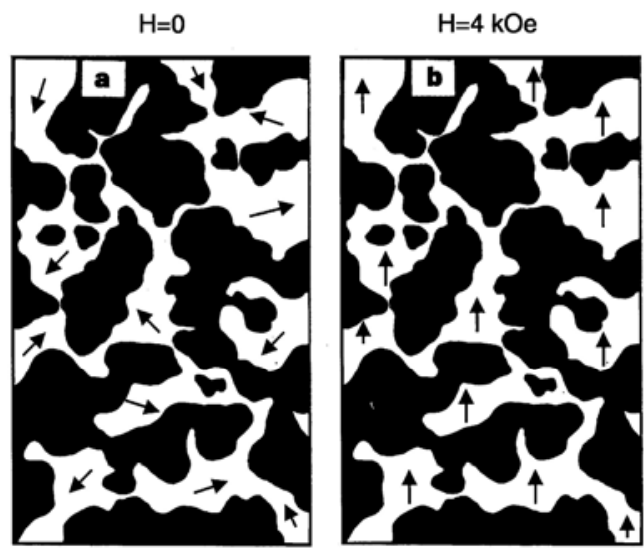

Abbildung 2.15: Schematische Darstellung der Verteilung von metallischen (weiß) und isolierenden (schwarz) Bereichen nach Uehara [50]. Zusätzlich sind magnetische Domänenausrichtungen dargestellt, die unter Feldeinfluss ausgerichtet werden.

Schematisch ist eine Verteilung, wie von Uehara [50] gezeigt möglich (Abbildung 2.15). Es existieren metallische Bereiche neben isolierenden Phasen. Während ohne Feld die ferromagnetischen Phasen ungeordnet vorliegen, besteht eine Ausrichtung der Domänen beim Anlegen eines magnetischen Feldes. Die Ergebnisse von Uehara zeigen jedoch, dass die entstehenden Phasen die Größenordnung von wenigen Nanometern überschreiten können und auch im Submikrometerbereich anzusiedeln sind. Moreo [8] versucht diese Bildung von großen, zusammenhängenden Clustern mit gleicher elektronischer Dichte unter Zuhilfenahme eines weiteren Parameters zu erklären. So wird von ihm die real existierende Unordnung durch chemische Variation der Komponenten in $\mathrm{La}_{1-\mathrm{x}} \mathrm{Ca}_{x} \mathrm{MnO}_{3}$ berücksichtigt. Hier werden Lanthandurch Calcium-Atome mit unterschiedlicher Elektronenkonfiguration und differierendem Ionenradius ersetzt. Der Einfluss dieser Änderung ist in Abbildung 2.16 skizziert. Die Abweichungen der Ladungsverteilung bzw. des Ionenradius gehen mit einer Änderung des Bindungswinkels der $\mathrm{Mn}-\mathrm{O}-\mathrm{Mn}$ Brücke einher und haben somit Einfluss auf das Sprungverhalten der Mangan $e_{g}$-Elektronen. Auf diese Weise entsteht eine Form der intrinsischen Unordnung. 


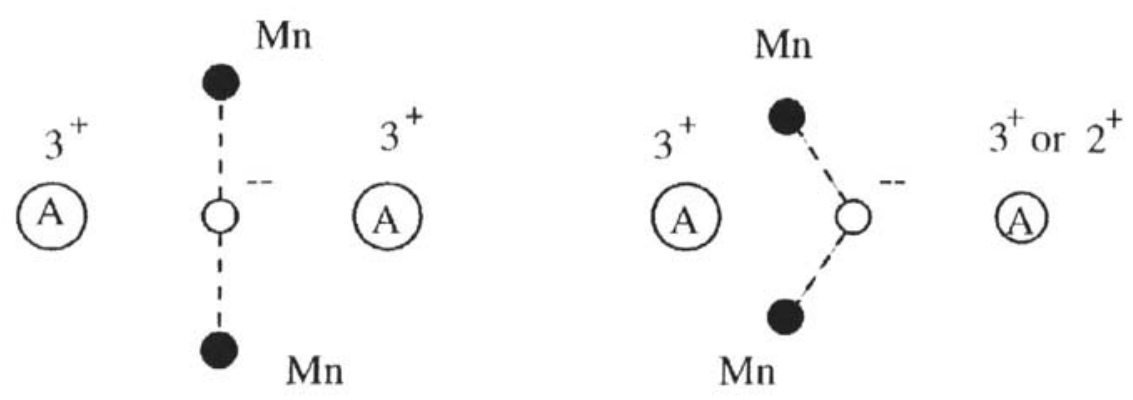

Abbildung 2.16: Schematische Darstellung des Ioneneinflusses auf den Mn - O - Mn Bindungswinkel. Dies hat eine Änderung des Sprungverhaltens der $e_{g}$ - Elektronen zur Folge [44].

Um diese Vorstellung an dem ein und zwei Orbitalmodell nachzuvollziehen, nimmt Moreo die Monte Carlo Simulation zur Hilfe und fügt dem Modell die intrinsische Unordnung als definierte Zufallskomponente $\delta$ hinzu. Als ein Ergebnis dieser Arbeit zeigt sich, dass sich nun in beiden Modellen homogene Bereiche ausbilden, deren Reichweite viel größer ist als der gewählte Netzabstand zwischen den Simulationszellen (Abbildung 2.17). Erkennbar ist, dass die Bereichsgröße direkt von der Zufallskomponente $\delta$ abhängt. Große Werte von $\delta$ erzeugen kleinere Cluster - kleine Werte von $\delta$ indes können aber zu recht großen Bereichen gleicher magnetischer Ordnung führen.

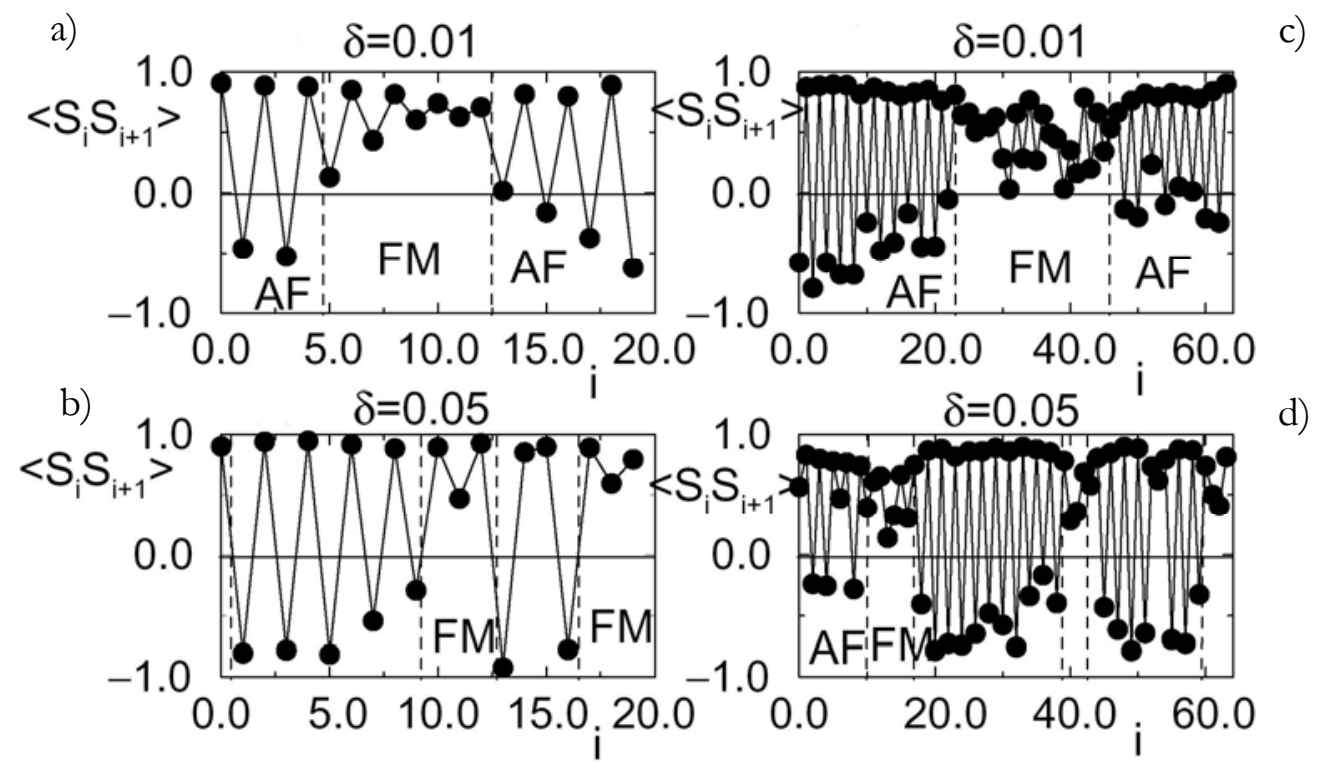

Abbildung 2.17: Monte Carlo Ergebnisse für das ein Orbitalmodell (rechts) und zwei Orbitalmodell (links) [8]. Aufgetragen ist die Spinkorrelation $\left\langle S_{i} S_{i+1}\right\rangle$ als Funktion der Korrelationslänge $i$. Die obere Reihe besitzt im Gegensatz zur unteren eine schwächere Zufallskomponente $\delta$. In allen vier Fällen ist eine größere Clusterbildung zu beobachten. 
In diesem Fall würde die Bildung von mehreren Grenzflächen dem System viel Energie kosten. Als Kompromiss bilden sich größere Cluster und nur wenige Trennflächen aus. Die bisher unternommenen Betrachtungen waren rein eindimensional und beschränkten sich auf eine geringe Reichweite von maximal 60 Gitterplätzen. Nun stellt sich die Frage, wie sich ein zweioder drei-dimensionales System verhält. Moreo greift deshalb auf das Ising Modell [51] zurück. Es ist ein Modell für auf einem Gitter wechselwirkende Spins und die daraus resultierenden magnetischen Eigenschaften des Vielteilchensystems. Dabei wird von einem regelmäßigen Gitter ausgegangen, auf welchem Spins mit den Zuständen $s_{i}=\{+1 ;-1\}$ definiert sind. In die Berechnung der Gesamtenergie mit der Hamiltonfunktion $H$ geht eine Summation über alle Nachbarpaare $\langle i, j\rangle$ sowie im allgemeinen Fall eine Summation über alle Gitterplätze $i$ ein.

$$
H=-J \sum_{\langle i, j\rangle} s_{i} s_{j}-h \sum_{i} s_{j}
$$

$h$ beschreibt den Einfluss eines äußeren Magnetfeldes, für $h=0$ bzw. $h \neq 0$ spricht man vom homogenen bzw. inhomogenen Ising-Modell. $J$ bezeichnet den Kopplungsparameter, der die Wechselwirkung zwischen benachbarten Spins $i$ und $j$ darstellt. Dieser ist bei einem regelmäßigen Gitter und der Einschränkung, dass Wechselwirkungen nur nächste Nachbarn betreffen, für alle Paare gleich. Zusätzlich gilt, dass bei negativem Vorzeichen des Kopplungsparameters statt eines ferromagnetischen ein antiferromagnetisches Modell simuliert wird. Die beiden Terme aus Gleichung (2.3) lassen sich folgendermaßen erklären:

1. Der erste Term beschreibt, dass ein System immer versucht, sich einheitlich einzustellen. Ferromagnetische Wechselwirkungen zwischen Spins zeigen bei tiefen Temperaturen entweder „up - spin“ oder „down - spin“ Verhalten. Grenzflächen zwischen zwei unterschiedlichen Domänen erhöhen die Energie und werden somit vermieden.

2. Die Summe im zweiten Term beschreibt den Ordnungszustand des Systems. Durch diesen Parameter wird die Bildung von kleineren Clustern beschrieben. Je größer die Unordnung, desto kleiner sind die zu erwarteten Bereiche. Über den Parameter $h$ kann ein äußeres Feld Einfluss auf den Ordnungszustand nehmen. 

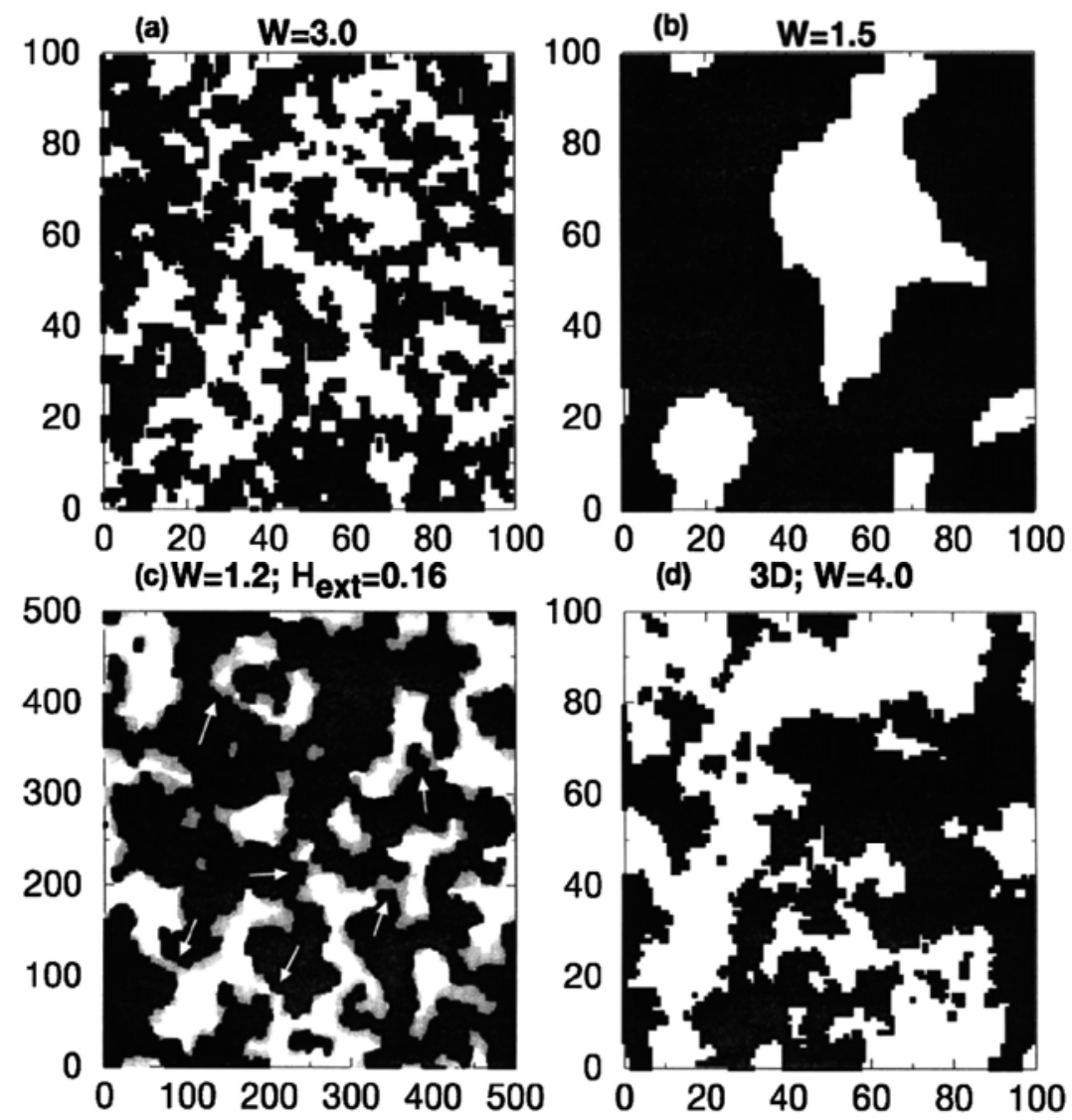

Abbildung 2.18: Ergebnisse der Monte Carlo Simulation mit Ising-Modell von Moreo [8]. In (a) und (b) wurden Cluster von je $100 \times 100$ Gitterpunkten berechnet, einzig der Parameter $W$ unterscheidet sich. Er definiert das Intervall [-W; W], aus dem zufällig chemische Potentiale $\mu$ ausgewählt werden. Ein hoher Wert für $W$ stellt somit ein größeres Maß der Unordnung, induziert über das chemische Potential $\mu$, dar. Das Ergebnis eines dreidimensionalen Netzwerkes ist in (d) dargestellt. Die Clustergrößenverteilung ist deutlich heterogener als in (a) oder (b). Bei Aktivierung eines Feldes wechseln einige Spins ihre Ausrichtung (grau dargestellt in c)).

Aus dem Wettbewerb dieser beiden konkurrierenden Bereiche zeichnet sich eine Bildung von Clustern unterschiedlicher Größe, wie in Abbildung 2.18 dargestellt, ab. Der Einfluss der intrinsischen Komponente zeigt deutliche Größenunterschiede bei der Clusterbildung in Abbildung (a) und (b). Wird zusätzlich ein externes Feld einbezogen, so wechseln einige Spins ihre Richtung (graue Flächen in (c)). Die schwarz dargestellte Fläche schrumpft zugunsten der weißen. Die Pfeile weisen auf Bereiche hin, bei denen unter Feldeinfluss Übergänge entstehen können - zwei getrennte Bereiche wachsen zu einem zusammen. Diese Übergangsform bezeichnet man als perkolativ ${ }^{3}$.

\footnotetext{
${ }^{3}$ Aus dem Lateinischen: percolare - durchdringen
} 
In den bisherigen Betrachtungen wurde der Temperatur als Variable bisher wenig Beachtung geschenkt. Die Simulationen wurden bei tiefen, festgehaltenen Temperaturen durchgeführt. Die Ergebnisse von v. Helmholt [1] an $\mathrm{La}_{2 / 3} \mathrm{Ba}_{1 / 3} \mathrm{MnO}_{\mathrm{x}}$ zeigen eine stark ausgeprägte Temperaturabhängigkeit des Transportwiderstandes (Abbildung 2.19). In Abbildung (a) ist der Widerstandverlauf mit und ohne Feldeinfluss einer nicht ausgelagerten Probe dargestellt. Er nimmt von tiefen Temperaturen her zu, erreicht ein Maximum und fällt bei höheren Temperaturen wieder ab. Unter Feldeinfluss verringert sich das Maximum und verschiebt sich zu höheren Temperaturen. Dies ist mit den theoretischen Ergebnissen aus Abbildung 2.18 (c) konform. Durch Anlegen von magnetischen Feldern werden einerseits die ferromagnetischen Domänen ausgerichtet und erniedrigen so den Transportwiderstand, zusätzlich entstehen durch Perkolation weitere metallisch leitfähige Kanäle die ebenfalls zu einer Widerstandssenkung führen und das Maximum zu höheren Temperaturen verschieben können.

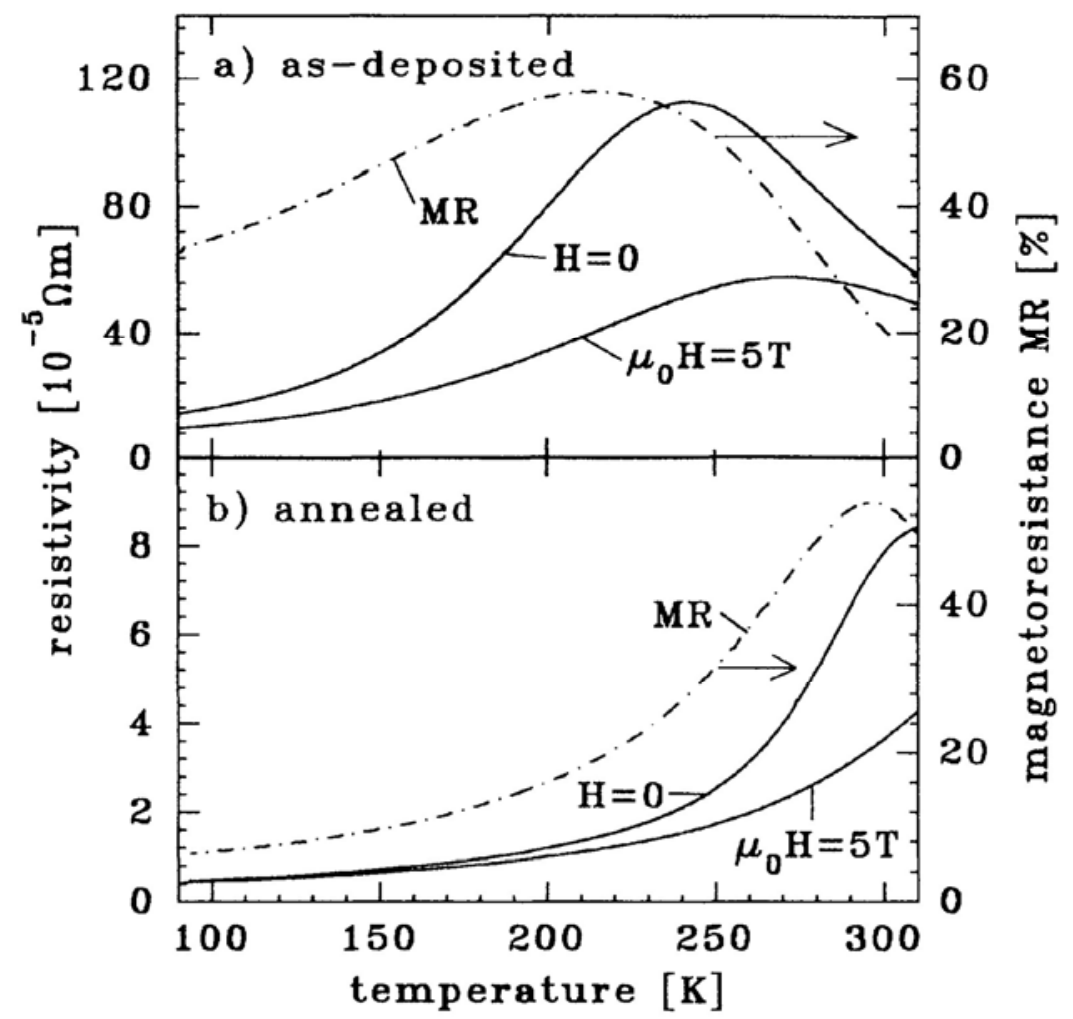

Abbildung 2.19: Temperaturabhängige Transportmessungen [1] an $\mathrm{La}_{2 / 3} \mathrm{Ba}_{1 / 3} \mathrm{MnO}_{\mathrm{x}}$ zeigen in (a) ein Maximum bei ca. 240K. Durch Anlegen eines Feldes $\left(\mu_{0} H=5 T\right)$ sinkt der Widerstand. Nach Auslagern der Probe bei $900^{\circ} \mathrm{C}$ für 12 Stunden sind beide Maxima zu höheren Temperaturen verschoben (b). 
Der Widerstandsverlauf bei tiefen Temperaturen gleicht dem von Metallen (er liegt jedoch weitaus höher!). Oberhalb des Metall - Isolator - Übergangs (MIT) besitzt er halbleitenden oder isolierenden Charakter. Um eine temperaturabhängige Simulation durchzuführen, nutzt Mayr diesen Vergleich als Ausgangspunkt seiner Überlegungen [6].

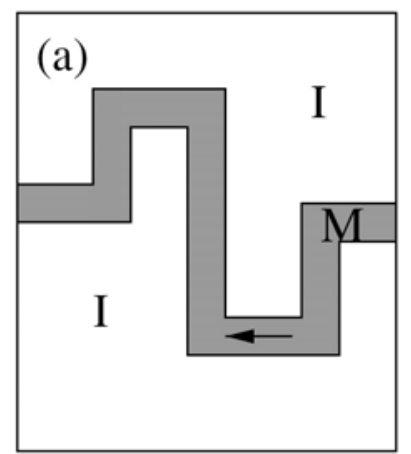

(b)

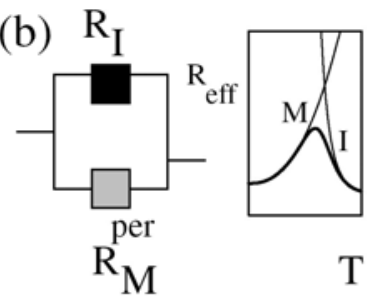

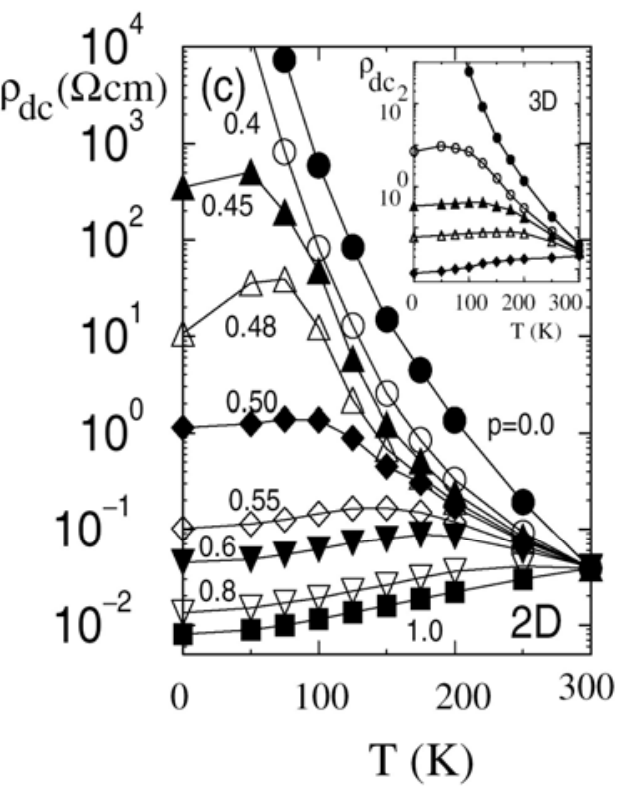

Abbildung 2.20: a) Schematische Darstellung eines perkolativen, metallischen Pfades innerhalb einer isolierenden Umgebung. Der Stromfluss wird durch Pfeile angedeutet.

b) Zwei - Widerstands - Modell für Manganate: Entsprechend der Darstellung in a) wird eine Parallelschaltung eines metallischen und eines isolierenden Widerstandes gebildet. Rechts ist das Ergebnis für den zu erwartenden Gesamtwiderstand dargestellt.

c) Widerstandsverlauf eines aus 100 x 100 Punkten bestehenden Widerstandsnetzwerkes für verschiedene Metall / Isolator Verhältnisse p. Der Einsatz zeigt die Simulationsergebnisse für ein Cluster mit $20^{3}$ Widerständen [6].

Nahe der Perkolation bei tiefen Temperaturen kann sich ein metallischer Pfad in einer isolierenden Matrix befinden (Abbildung 2.20 (a)). Dieser erlaubt den elektrischen Kontakt durch die Probe hindurch. Wird die Temperatur erhöht, steigt der Widerstand des metallischen Pfades an, wohingegen der des Isolators sinkt. Eine vereinfachte Schaltung für diese Situation ist in Abbildung 2.20 (b) dargestellt. Es handelt sich um zwei parallel geschaltete Widerstände. Diese Schaltung verhält sich entsprechend der oben beschriebenen Ausgangssituation und lässt einen Widerstandsverlauf entsprechend (b) zu. In (c) ist das Ergebnis der Widerstandsnetzwerk - 
Berechnungen ${ }^{4}$ von Mayr dargestellt. Bei einem Metall / Isolator Verhältnis von $p=0.45$ bis $p=$ 0.6 ist ein isolierendes Verhalten nahe Raumtemperatur zu erkennen. Zu tieferen Temperaturen hin, weisen die jeweiligen Graphen ein Maximum auf, gefolgt von metallischen Widerstandsverläufen.
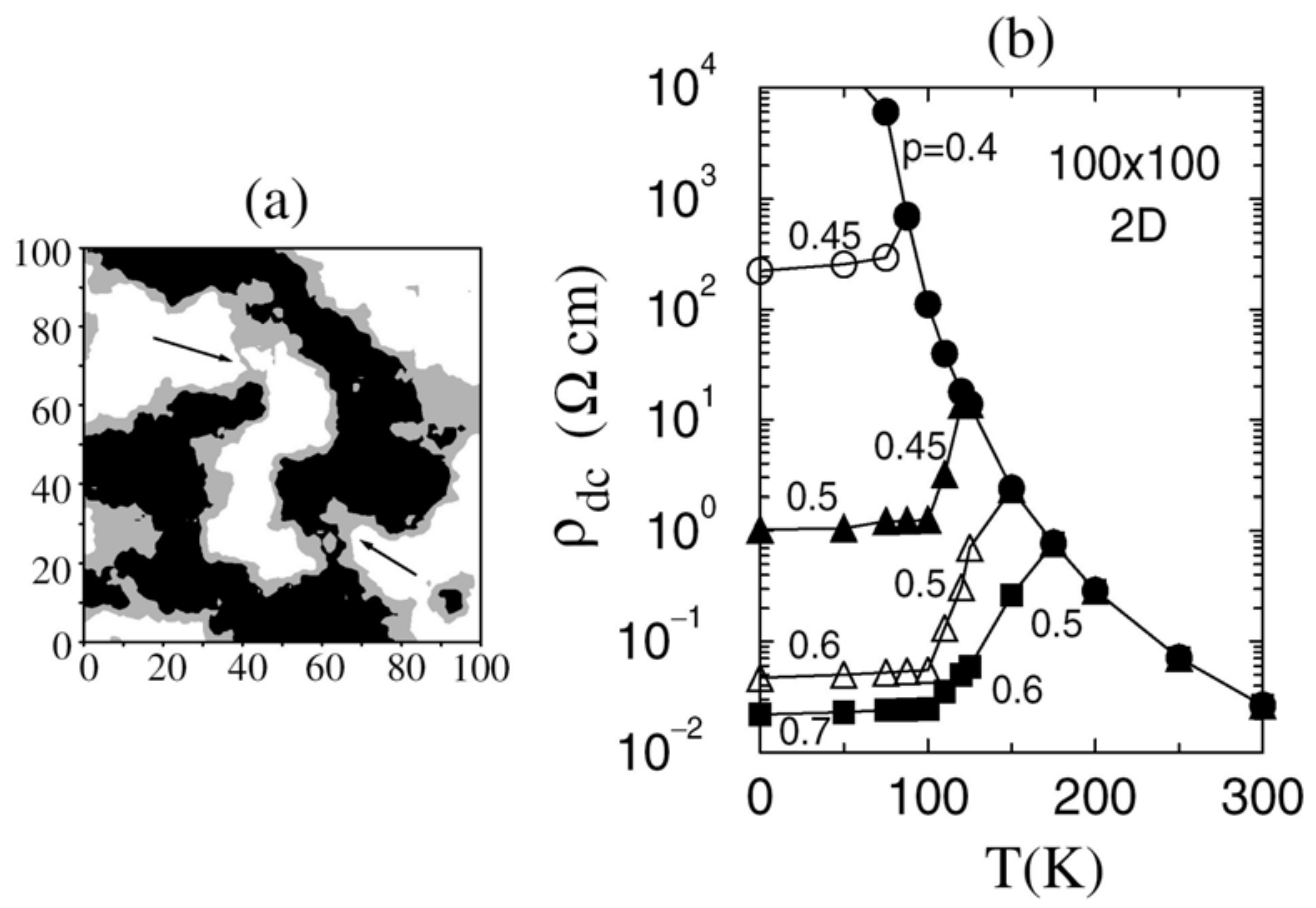

Abbildung 2.21: a) Ergebnis einer Monte Carlo Simulation bei fester Temperatur: In schwarzen (weißen) Regionen sind die Spins nach oben (unten) ausgerichtet. Bei beiden liegt Ferromagnetismus vor. Die grau dargestellten Bereiche sind temperaturinduziert und isolierend. Eine Temperaturänderung kann bei den mit Pfeilen markierten Stellen zu perkolativen Übergängen führen.

b) Temperaturabhängiger Widerstandsverlauf eines zweidimensionalen $100 \times 100$ Widerstandsnetzwerkes. Das Metall/Isolatorverhältnis $p$ ist temperaturabhängig [6].

In Abbildung 2.21 (a) ist das Ergebnis einer Monte Carlo Simulation bei fester Temperatur gezeigt. Weiße und schwarze Bereiche sind ferromagnetisch - beide unterscheiden sich lediglich in ihrer Spinausrichtung. Aufgrund des Temperatureinflusses werden paramagnetisch isolierende Bereiche (grau) gebildet, die metallische Pfade durchtrennen (siehe eingezeichnete Pfeile), und somit den Gesamtwiderstand erhöhen. Eine Erniedrigung der Temperatur verringert die Ausbildung der isolierenden Phase und führt zur Perkolation.

\footnotetext{
${ }^{4}$ Näheres zur Berechnungstechnik ist bei S. Kirkpatrick [9] erläutert.
} 
Um dieses Verhalten auch bei Widerstandsnetzwerken einfließen zu lassen, hat Mayr das Metall / Isolatorverhältnis empirisch temperaturabhängig gewählt. Mit zunehmender Temperatur sinkt der Anteil der metallischen Phase. Nahe $\mathrm{T}_{\mathrm{C}}$ geschieht dies, entsprechend den Ergebnissen aus Magnetisierungsmessungen an Manganaten, schneller als bei sehr tiefen Temperaturen. In Abbildung 2.21 (b) ist der somit entstehende Widerstandsverlauf dargestellt. Der simulierte Widerstand besitzt bei Temperaturen um 150K ein Maximum und fällt nach beiden Seiten ab. Ersichtlich ist, dass eine bessere Nachbildung des Widerstandsverlaufes entsprechend den aus Transportmessungen bekannten Kurven möglich ist. 


\section{Rastertunnelmikroskopie (STM) und - Spektroskopie (STS)}

\subsection{Theoretische Grundlagen}

Die Oberflächenforschung wurde 1982 durch eine Erfindung von Binnig und Rohrer [52; 53] um eine weitere Analysetechnik der Rastersondenmethoden bereichert. Das von ihnen entwickelte STM (Scanning Tunneling Microscope) ermöglichte Realraumabbildungen von lokalen elektronischen Strukturen sowie Atompositionen halbleitender und metallischer Oberflächen. 1986 wurden ihre Leistungen mit dem Physiknobelpreis ausgezeichnet.

a)

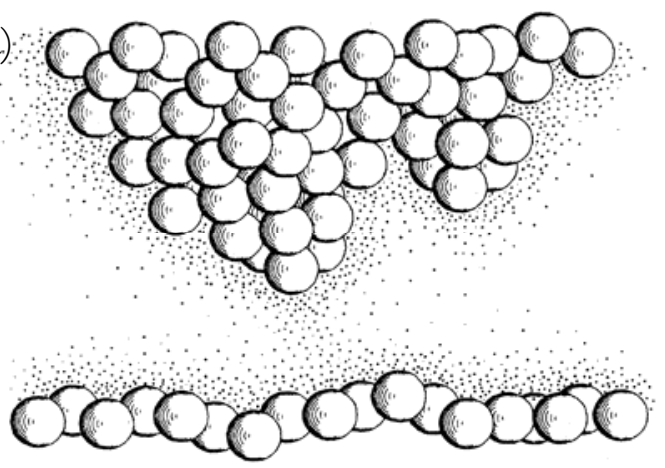

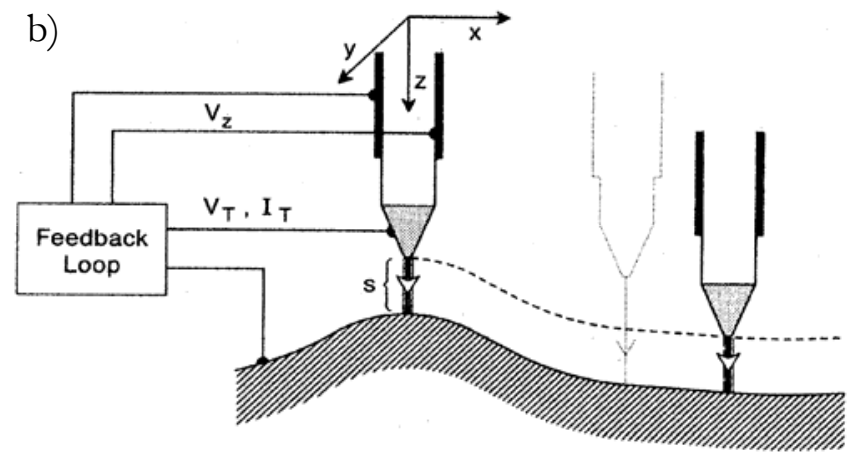

Abbildung 3.1: Prinzip des Rastertunnelmikroskops [54]

a) Die metallische Sonde ist nur wenige Angström von der Probe entfernt. Die kleinen Punkte deuten die Elektronendichte an. An der Stelle, an der sich Probe und Spitze am nächsten kommen, ist die Elektronendichte erwartungsgemäß am größten.

b) Die Spitze wird beim Rasterprozess immer im gleichen Abstand zur Probe gehalten. Hieraus ergibt sich später das topographische Bild

Das Funktionsprinzip des STM basiert auf einer atomar scharfen Sonde (im Idealfall aus einem einzigen Atom bestehend), die mit Hilfe von piezokeramischen Elementen mit subatomarer Genauigkeit ${ }^{5}$ im Raum positioniert werden kann. Hierbei kommen in der Regel drei orthogonal angeordnete Piezokristalle oder ein einzelner so genannter Röhrchenscanner, dessen Ausdehnung bzw. Verbiegung durch Anlegen von Spannungen variiert werden kann, zum Einsatz. Im Experiment wird als Sondenmaterial ein Stück Metalldraht (meist Wolfram oder $\mathrm{Pt}_{80} \mathrm{Ir}_{20}$ ) verwandt, der durch Schneiden, Zerreißen oder elektrochemisches Ätzen präpariert wird [55; 56; 57; 58]. Diese Spitze wird an der Rastereinheit befestigt und berührungslos über die zu

\footnotetext{
${ }^{5}$ Dies gilt, sofern die Drift in x- und y-Richtung vernachlässigt wird.
} 
untersuchende Oberfläche geführt (siehe Abbildung 3.1). Hierbei kann das Mikroskop in zwei verschiedenen Arbeitsmoden betrieben werden:

- Im so genannten „constant current mode“ versucht der Regler, den Tunnelstrom immer konstant zu halten. Hierzu wird über eine Rückkopplungsschleife die vertikale Position der Spitze über allen Positionen der zu untersuchenden Oberfläche so eingestellt, dass dieser Tunnelstrom erreicht wird. Der Piezokristall wird dem entsprechend gestaucht oder gestreckt. Der Abstand von Spitze und Probenoberfläche bleibt so konstant. Aus den Spannungswerten am Piezostellelement läst sich die Topographie der Oberfläche berechnen.

- Beim „constant height mode“ bleibt die vertikale Position der Spitze konstant. Es erfolgt hier im Gegensatz zum „constant current mode“ nur eine sehr schwache Höhennachregelung der Tunnelspitze zur Oberfläche. Die topographische Information erhält man hier aus dem variierenden Tunnelstrom. Dieser Modus sollte nur auf atomar glatten Oberflächen (z.B. Graphit) eingesetzt werden, da ansonsten die Gefahr besteht, dass die Spitze die Oberfläche der Probe berührt und somit beschädigt wird.

a)

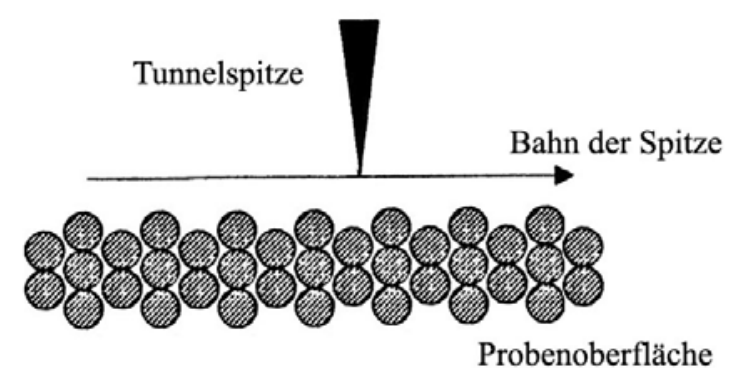

b)

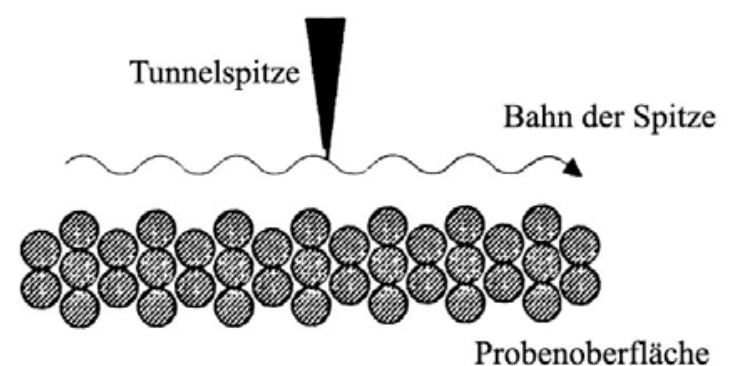

Abbildung 3.2: Schematische Darstellung von a) constant height mode und b) constant current mode

Der Abstand $s$ zwischen Spitze und Probe wird durch den quantenmechanisch zu erklärenden Tunneleffekt bestimmt. Elektronen können die schmale Potentialbarriere, die der Spalt zwischen den beiden Elektroden darstellt, durchdringen. Wird zwischen Probe und Spitze eine Spannung $U$ angelegt, so fließt bei hinreichend kleinem Abstand $s$ der Tunnelstrom $I$. Diese Lücke zwischen Spitze und Probe wird mit den vorgegebenen Werten für die Tunnelspannung $U$ (im Regelfall $\leq$ 1V) und den Tunnelstrom $I_{T}$ entsprechend

$$
I \propto U e^{-\left(\frac{2 \sqrt{2 m \Phi}}{\hbar} s\right)}
$$


eingestellt ( $\Phi$ : effektive lokale Barrierenhöhe; $m$ : Elektronenmasse) [59]. Die vorgegebene BiasSpannung bestimmt dabei, welche Elektronenzustände am Tunnelprozess teilnehmen. Somit können unterschiedliche Zustände der Oberfläche abgebildet werden, wie es bei der Untersuchung an Halbleitern häufig beobachtet wurde [60]. Das System aus Spitze und Probe ist jedoch sehr komplex und kann ohne vereinfachende Näherungen nur schwer dargestellt werden.

Eine erste, stark vereinfachte Theorie zur Beschreibung des STM stammt von Tersoff und Hamann [59]. Sie betrachteten den elastischen Anteil des Tunnelstroms und nutzten den Störungsansatz von Bardeen [61]. Hierzu wird eine zeitabhängige Störungsrechnung erster Ordnung für das System aus Spitze und Probe durchgeführt. Es wird vorausgesetzt, dass die Wellenfunktionen von Spitze und Probe nur schwach miteinander wechselwirken und somit die Wellenfunktionssysteme beider unabhängig bestimmt werden können. Für den Tunnelstrom $I$ gilt:

$$
I=\frac{2 \pi e}{\hbar} \sum_{\mu, v} f\left(E_{\mu}\right)\left[1-f\left(E_{v}+e U\right)\right]\left|M_{\mu \nu}\right|^{2} \delta\left(E_{\mu}-E_{v}\right)
$$

Dabei sind $E_{\mu}$ und $E_{v}$ die Energieeigenwerte der Wellenfunktionen der Spitze bzw. der Probe, $f(E)$ ist die Fermi - Funktion und bei $U$ handelt es sich um die Spannung zwischen der Spitze und der Probe. Wenn man nun die Temperatur und Bias-Spannung sehr klein wählt, vereinfacht sich (3.2) zu:

$$
I=\frac{2 \pi}{\hbar} e^{2} U \sum_{\mu, v}\left|M_{\mu v}\right|^{2} \delta\left(E_{v}-E_{F}\right) \delta\left(E_{\mu}-E_{F}\right)
$$

Das Übergangsmatrixelement $M_{\mu v}$, das die Überlappung von Spitze $\Psi_{\nu}$ und Probe $\Psi_{\mu}$ beschreibt, kann laut Bardeen $[61,62]$ über die Trennfläche zwischen Probe und Spitze berechnet werden:

$$
M_{\mu \nu}=-\frac{\hbar^{2}}{2 m} \int\left(\psi_{\mu}^{*} \vec{\nabla} \psi_{\nu}-\psi_{\nu} \vec{\nabla} \psi_{\mu}^{*}\right) d \vec{S}
$$

Die geometrische und elektronische Struktur der Spitze ist jedoch unbekannt. Um die Lösung des Integrals zu vereinfachen, wählen Tersoff und Hamann für die Spitze ein lokales, sphärisches Potential entsprechend eines $s$ - Orbitals (Abbildung 3.3). Die Austrittsarbeiten von Spitze und Probe werden zusätzlich als gleich angesehen. 


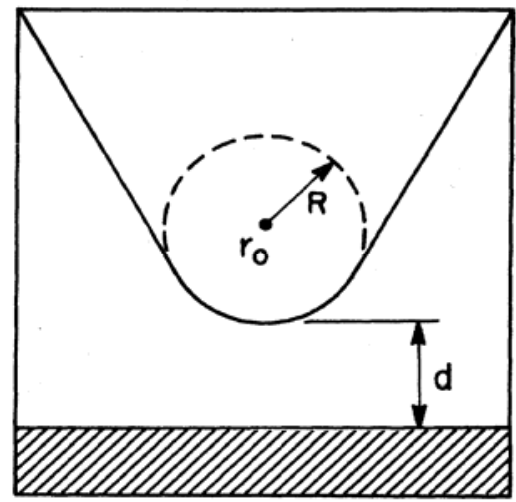

Abbildung 3.3: Schematische Darstellung der Tunnelgeometrie nach [59]

Als Ergebnis dieser Theorie ergibt sich ein Zusammenhang zwischen Tunnelstrom und der elektronischen Zustandsdichte der Probe am Ort $\vec{r}_{0}$. Die Tunnelleitfähigkeit wird bestimmt zu:

$$
\sigma \approx 0.1 R^{2} e^{2 \kappa R} \rho\left(\vec{r}_{0}, E_{F}\right)
$$

mit $\kappa$ als Abklingkonstante der Wellenfunktion der Probe:

$$
\kappa=\frac{\sqrt{2 m \Phi}}{\hbar}
$$

und einer Zustandsdichte von:

$$
\rho\left(\vec{r}_{0}, E_{F}\right)=\sum_{v}\left|\psi_{v}\left(\vec{r}_{0}\right)\right|^{2} \delta\left(E_{v}-E_{F}\right)
$$

Topographische Aufnahmen, gewonnen durch STM - Messungen, entsprechen laut dem Ergebnis von Tersoff und Hamann dem Abbild der lokalen Zustandsdichte. Dies gilt aber nur so lange, wie die Austrittsarbeit oder die Geometrie des Tunnelbereichs nicht geändert wird. Zusätzlich kann zur Interpretation der topographischen Bilder die Rastertunnelspektroskopie (STS) herangezogen werden. An fest definierten Stellen auf der Probenoberfläche werden so genannte Kennlinien aufgezeichnet, bei denen der Tunnelstrom als Funktion der Spannung gemessen wird. Der Abstand zwischen Spitze und Probe wird durch die so genannte „sample and hold“ - Schaltung konstant gehalten. Unter der Voraussetzung, dass das Spitzenmaterial aus einem Metall mit freien Elektronen besteht, bzw. die Zustandsdichte nahe der Fermienergie sich 
nicht stark ändert, kann man eine Aussage über die elektronische Zustandsdichte ${ }^{6}$ am Ferminiveau der Probe treffen [55; 62].

$$
\frac{d I}{d U} \approx \rho_{v}\left(E_{F}-e U\right)
$$

Die Zustandsdichte der Probe ist in dieser Nährung proportional zur Leitfähigkeit $d I / d U$. Bei Metallen ist dem Ohmschen Gesetz entsprechend eine linear verlaufende Kennlinie nahe $0 \mathrm{~V}$ mit einer endlichen Steigung zu erwarten. Halbleiter hingegen zeigen aufgrund einer vorliegenden Bandlücke diese Abhängigkeit nicht. Die lokale Zustandsdichte (LDOS) ist stark energieabhängig, besonders an den Bandkanten ändert sie sich diskontinuierlich. Die bei positiven und negativen Spannungen abgebildeten LDOS können sich stark voneinander unterscheiden (Abbildung 3.4). Es kann zusätzlich eine Energielücke nahe $0 \mathrm{~V}$ vorliegen, welche sich durch eine ebene Kennlinie im I-U Diagramm auszeichnet.

(a)

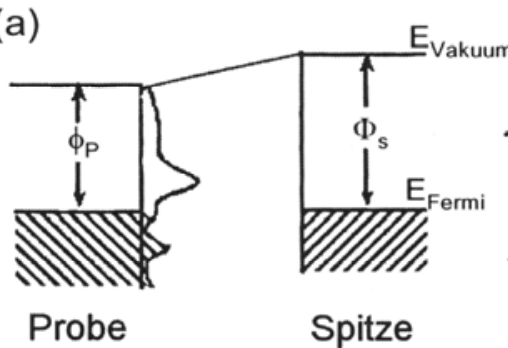

(b)

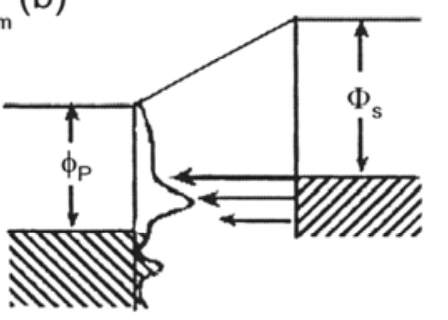

(c)

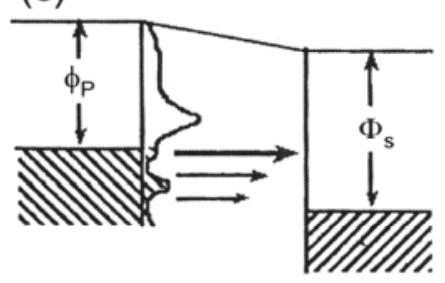

Abbildung 3.4: a) Stromlos

b) Elektronen tunneln von der Spitze in die Probe

c) Elektronen tunneln von der Probe in die Spitze

Durch die Verschiebung der Leitungsbänder aufgrund der angelegten Tunnelspannung werden unterschiedliche Zustände abgebildet [55].

\footnotetext{
${ }^{6}$ Engl.: density of states - DOS
} 


\subsection{Die Messapparatur}

Zur Messung von STS bzw. STM kommt ein Gerät der Firma Omicron ${ }^{7}$ zum Einsatz. Es handelt sich um ein $\mathrm{VT}^{8}$-STM, dass Messungen in einem Temperaturbereich von $25 \mathrm{~K}$ bis $1500 \mathrm{~K}$ im Ultrahochvakuum (UHV) ermöglicht. Durch die Kombination von federnder Aufhängung und Wirbelstrombremsen zur Dämpfung mechanischer Schwingungen kann man nahezu störungsfrei rastertunnelmikroskopische Analysen vornehmen (Abbildung 3.5).

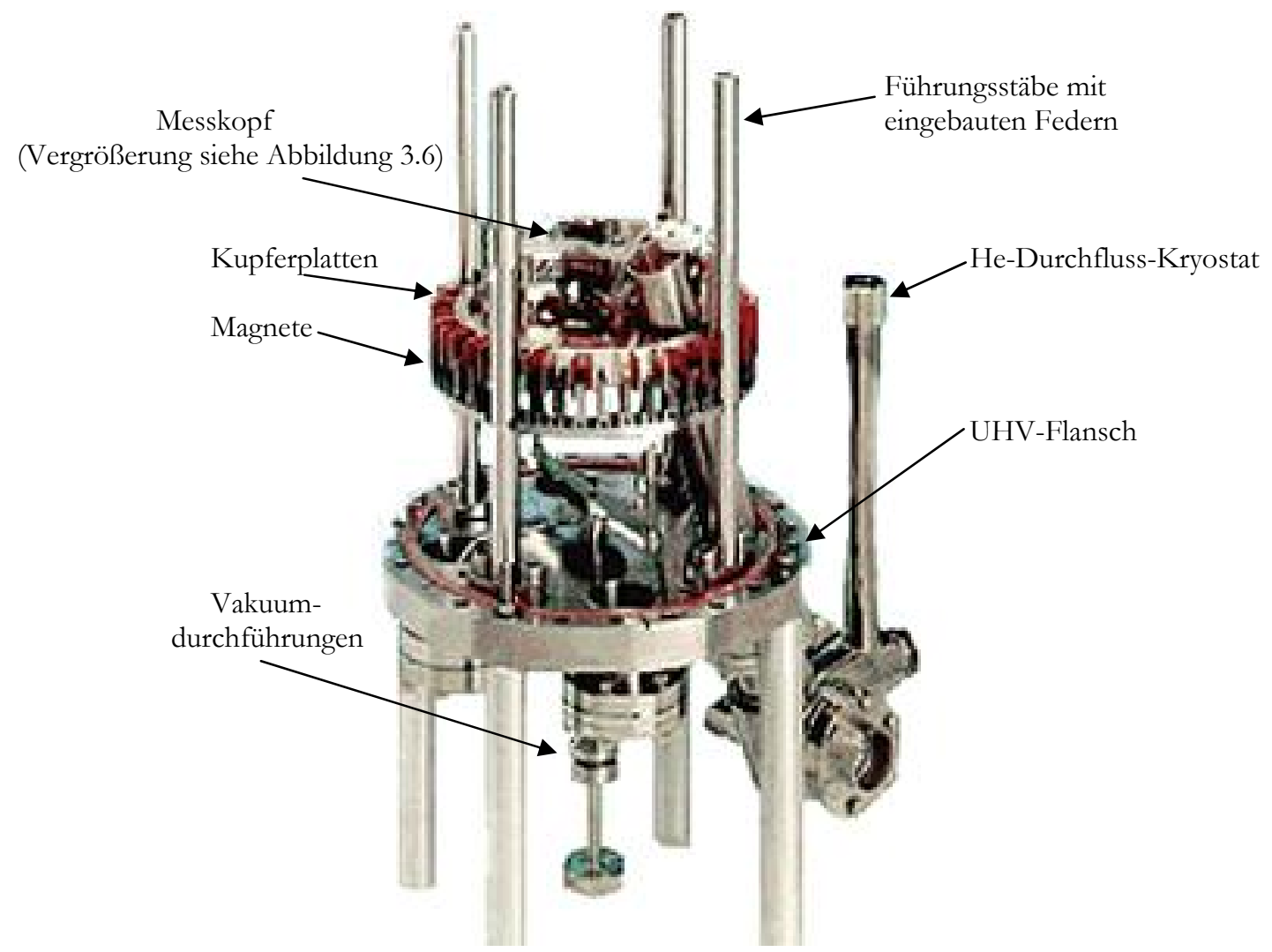

Abbildung 3.5: Bild der STM - Bühne (Fa. Omicron), die Wirbelstromdämpfung, bestehend aus Kupferplatten und Magneten, ist erkennbar

Die Anlage verfügt zusätzlich über eine durch Luftkissen realisierte Schwingungsdämpfung. Um eine Einkopplung von Gebäudeschwingungen zu verhindern, steht die Apparatur auf einem, vom Fundament entkoppelten massiven Betonsockel.Thermische Drift, elektronische Störungen sowie akustische Einkopplungen können nicht ausgeschlossen werden und begleiten die rastermikroskopischen Messungen.

\footnotetext{
${ }^{7}$ Omicron NanoTechnology GmbH, Limburger Str. 75, 65232 Taunusstein, Germany www.omicron.de

${ }^{8}$ variable temperature
} 


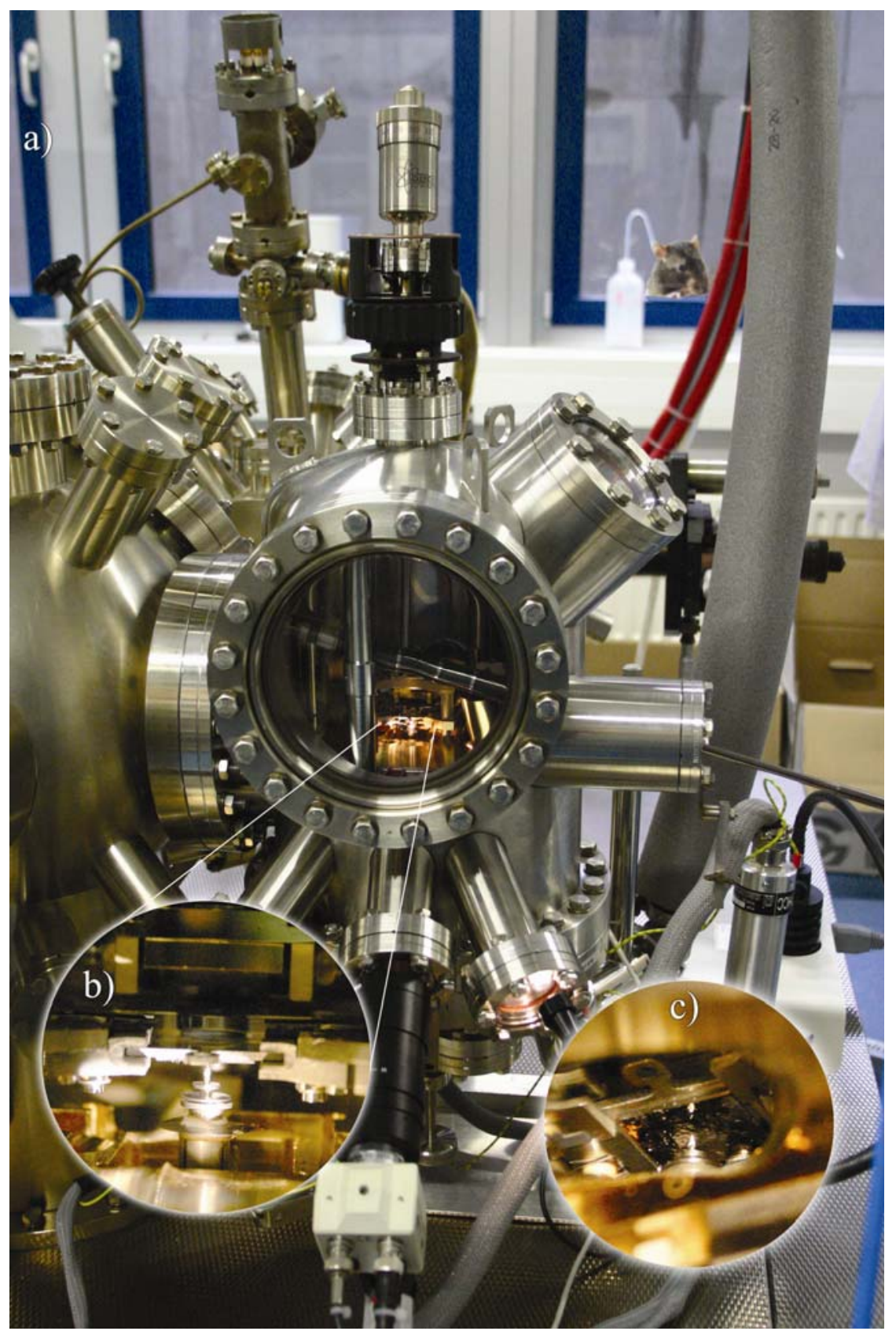

Abbildung 3.6: a) UHV-Messkammer mit dem VT-STM

b) Vergrößerung der Scannereinheit - Zu sehen sind im unteren Bereich der Piezoscanner mit der Spitze und darüberliegend die Probe. Im Oberen Bereich befindet sich der so genannte „Clampingblock“, er dient zur thermischen Ankopplung der Probe an den Kryostaten.

c) Blick von unten auf die Spitze und Probe - die Reflexion der Spitze ist zu erkennen

Das Mikroskop besteht aus einem Röhrchenscanner, auf dem die Spitzenhalter mit Hilfe eines Permanentmagneten befestigt werden. Die Scaneinheit kann über Scherpiezos grob in x-, y- und z-Richtung bewegt werden. Oberhalb des Scanners befindet sich mit der Probe nach unten zeigend der Probenträger (Abbildung 3.6). Über den „Clamping Block“ kann dieser thermisch an 
den Durchflusskryostaten gekoppelt werden. Da zur Ankopplung ein Bündel vergoldeter Kupferdrähte eingesetzt wird, liegt die minimal erreichbare Temperatur an der Probe bei ca. $25 \mathrm{~K}$.

Durch ein Nadelventil kann der Durchfluss des Kühlmittels (flüssiges Helium) reguliert und somit die Temperatur grob eingestellt werden. Eine genauere Justage ist durch einen Heizer, der von einem PID-Regler angesteuert wird, möglich. Eine Videokamera dient zur Kontrolle der manuellen Spitzenbewegung und bei der Annäherung der Spitze an die Probe (Abbildung 3.6 (c)). 


\section{Auswerteverfahren}

\subsection{Analysemethode der Tunnelspektren}

Im nun folgenden Kapitel wird die Analysemethode der gemessenen Tunnelspektren vorgestellt. Wie bereits in Kapitel 3 erläutert, gilt die Steigung der STS - Tunnelkennlinie nahe $0 V$ als Maß für die lokale Zustandsdichte und stellt somit die Tunnelleitfähigkeit des untersuchten Bereiches der Probe dar. In Abbildung 4.1 sind zwei unterschiedliche Spektren exemplarisch abgebildet, die an einer gesputterten, $90 \mathrm{~nm}$ dicken $\mathrm{La}_{0.7} \mathrm{Ca}_{0.3} \mathrm{MnO}_{3}$ (LCMO) - Schicht auf $\mathrm{SiO}_{2}$ unter UHV Bedingungen gemessen wurden.

Die schwarz dargestellte Kurve in Abbildung 4.1 wurde bei $50 \mathrm{~K}$ aufgenommen und zeigt nahe $0 \mathrm{~V}$ eine endliche Steigung. Der rote Graph hingegen weist ein isolierendes Verhalten auf, das sich durch eine waagerecht verlaufende Kennlinie im gleichen Bereich auszeichnet.

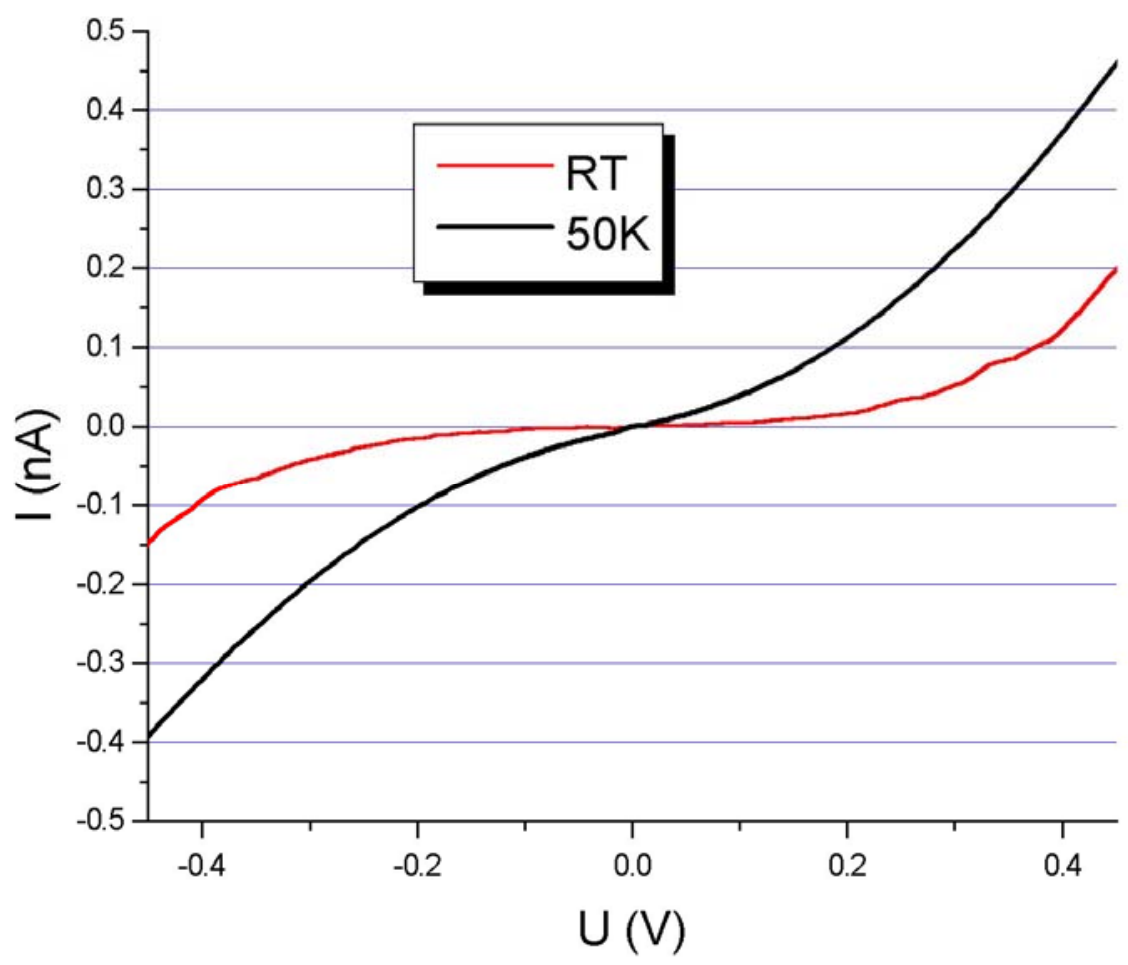

Abbildung 4.1: Tunnelkennlinien von metallisch leitfähigen und isolierenden Bereichen; gemessen bei unterschiedlichen Temperaturen 
Nun stellt sich die Frage, ob sich dieser Verlauf der Kennlinien über einen größeren Probenausschnitt hinweg konstant verhält, oder ob Unterschiede vorhanden sind: Existieren leitfähige metallische Bereiche neben isolierenden Gebieten bei einer Temperatur? Um diese Frage zu klären, wird auf das Hilfsmittel der gerasterten Kennlinienanalyse zurückgegriffen.

Der zur Datenaufnahme des Tunnelmikroskops verwendete Computer nutzt unter Windows NT4.0 die auf IDL ${ }^{9}$ basierte Scala Software (Version 4.0). Über eine bidirektionale Firewire Schnittstelle (IEEE 1394) empfängt er die Messdaten, kann aber auch die gesamte STM Elektronik steuern. Da keinerlei externe Einstellungen bei diesem Gerät vorzunehmen sind, besteht die Möglichkeit, komplexe Messprogramme automatisiert durchführen zu lassen. Dieser Vorteil wird bei der Kennlinienaufnahme genutzt. Parallel zum topographischen constantcurrend-mode werden einem Raster entsprechend Punkte definiert, an denen Scala zusätzliche Tunnelspektren aufzeichnet. Hierbei besteht die Möglichkeit die Rasterweite in der x- bzw. yEbene zu definieren und zusätzliche Parameter [63] einzustellen.

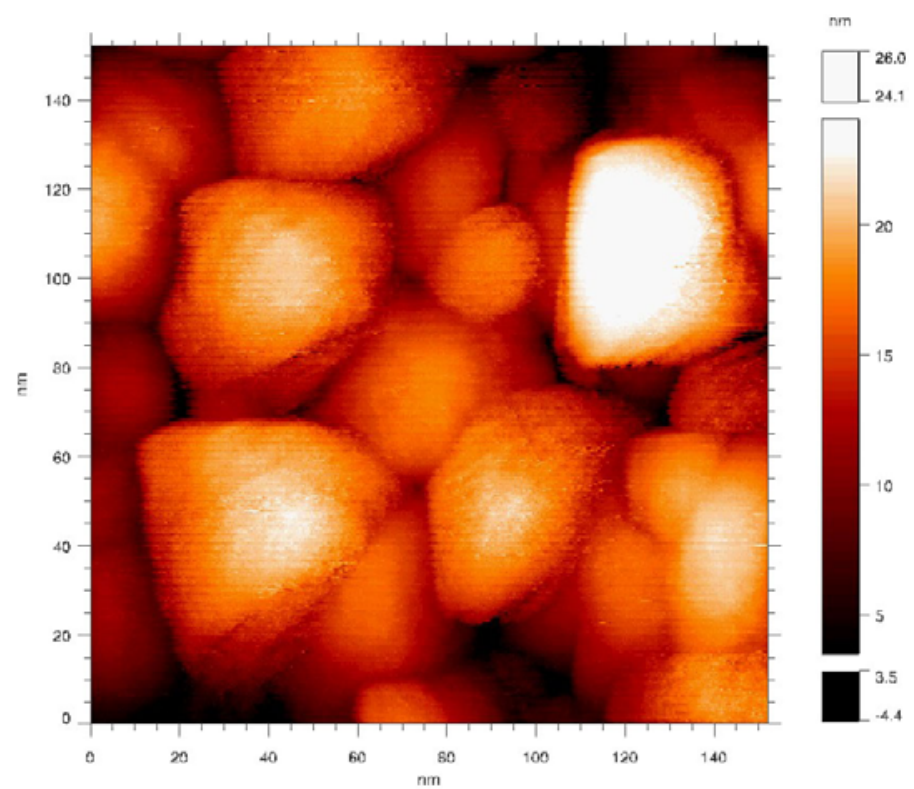

Abbildung 4.2: Topografische Darstellung der Oberfläche einer LCMO - Schicht (50 nm präpariert durch $\mathrm{MAD}^{10}$ )

Im Folgenden soll die softwaregestützte Analysemethode an einem Beispiel erläutert werden. Hierzu wird das Ergebnis einer Messung an einem $50 \mathrm{~nm}$ dicken LCMO - Film (präpariert durch MAD[64]) bei $130 \mathrm{~K}$ herangezogen. Die gewonnenen topographischen Informationen bestehen aus $400 \times 400$ Bildpunkten (Abbildung 4.2). Definiert man ein $5 \times 5$ Raster auf dieser Oberfläche,

\footnotetext{
${ }^{9}$ RSI Research Systems Inc.- Germany: CREASO, GmbH, Talhofstrasse 32A, D-82205 Gilching www.creaso.com

${ }^{10}$ MAD: Metallorganische Aerosol Deposition [64; 71]
} 
so werden $80 \times 80$ Tunnelspektren von $-0,5 \mathrm{~V}$ bis $0,5 \mathrm{~V}$ mit je 200 Messwerten aufgezeichnet.

Aufgrund der extrem hohen Datenmengen wird diese Analyse automatisiert durchgeführt.
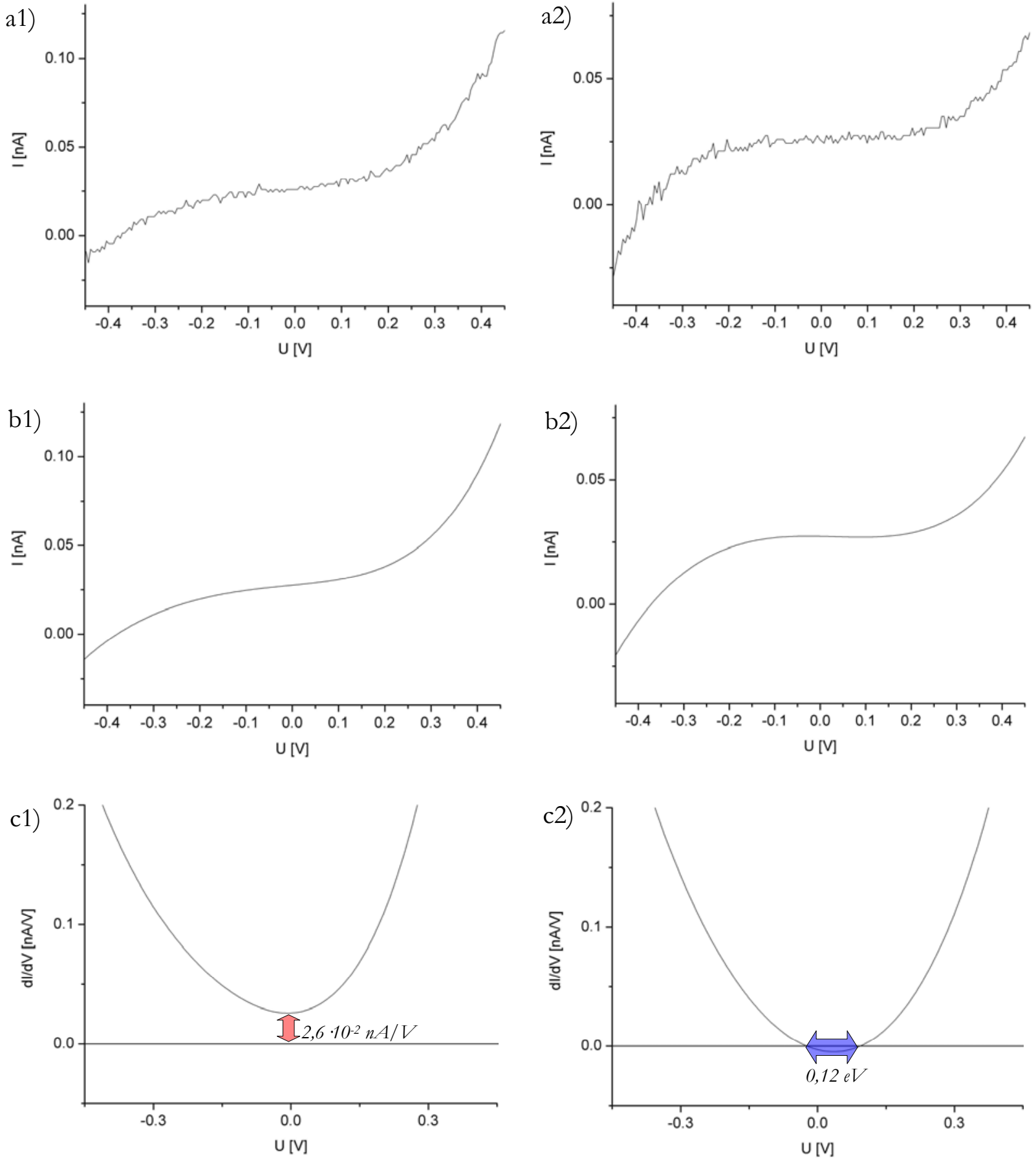

Abbildung 4.3: Beispiel zur automatisierten Kennlinienanalyse:

a1) und a2) Gemessene Kennlinie im Bereich von $-0,5 \mathrm{~V}$ bis 0,5 $\mathrm{V}$

b1) und b2) Gefittetes Polynom entsprechend a1) und a2)

c1) und c2) Ableitung des Polynoms aus b1) bzw. b2) mit Bestimmung der Leitfähigkeit in c1) bzw. der Breite der Energielücke c2) 
Zur Analyse der Kennlinien kommt wieder IDL zum Einsatz. Die aufgenommenen Spektroskopiedaten werden aus den Messfiles des Mikroskops importiert und in einem Array gespeichert. Um Ausreißer und Rauschen zu verringern, unterzieht man jede einzelne Kennlinie einem Glättungsalgorithmus. Es hat sich gezeigt, dass ein Polynomfit fünften Grades an die Spektroskopiedaten die besten Resultate liefert. Beispielhaft ist dies in Abbildung 4.3 dargestellt. In Abbildung $(\mathrm{a} 1+2)$ und $(\mathrm{b} 1+2)$ sind Kennlinien und die dazugehörigen Fitte zu sehen. Anschließend wird durch Differentiation die Ableitung der I/U - Kennlinie errechnet (Abbildung 4.3 (c)) und im Fall 1) der Abstand zwischen dem Minimum und der x - Achse bestimmt. Dieser Wert wird in eine Farbskala übersetzt und in eine so genannte Leitwertkarte eingetragen. Sobald dies für alle Kennlinien geschehen ist, kann man zusätzlich zu den morphologischen Informationen (Abbildung 4.2) auch eine Aussage über die ortsabhängige Tunnelleitfähigkeit treffen.

a)

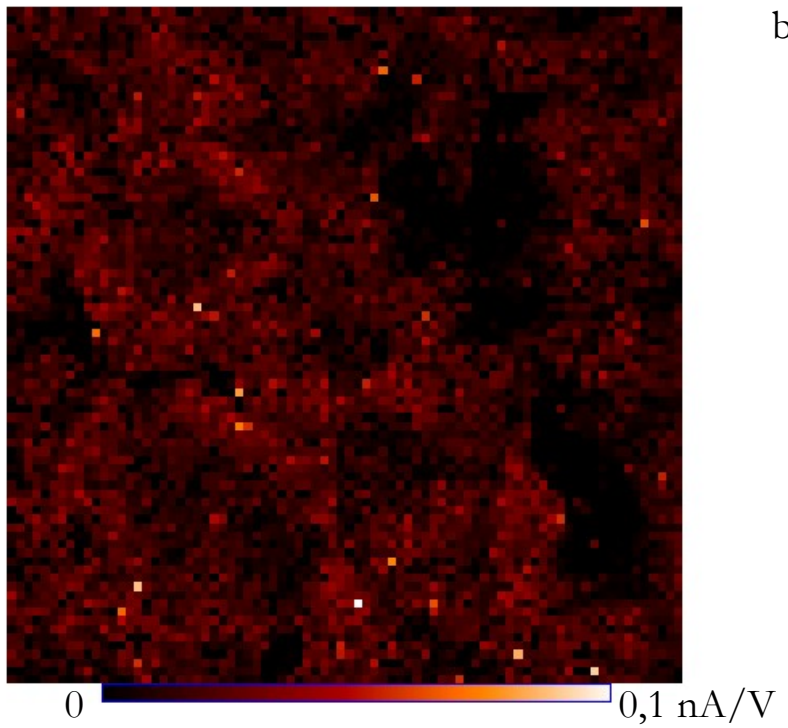

b)

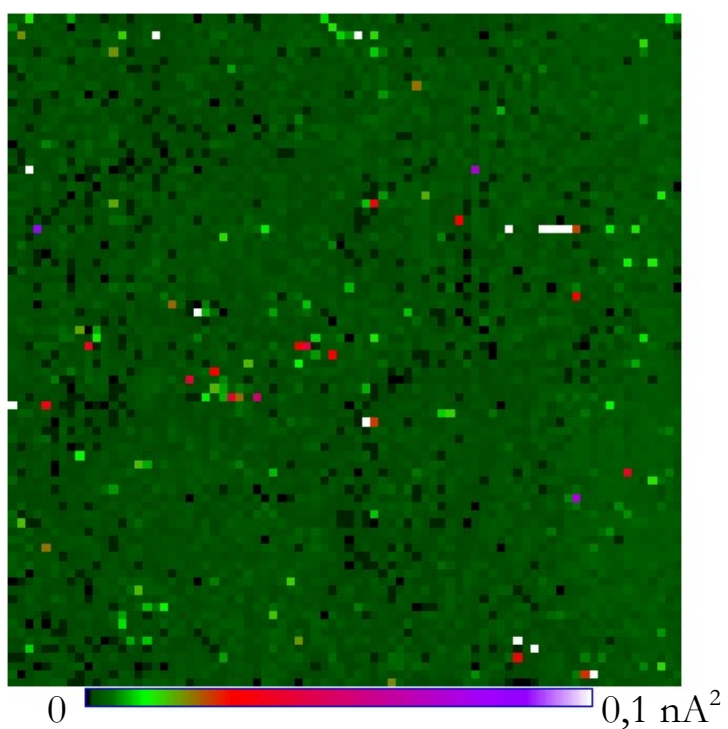

Abbildung 4.4: a) Leitwertmap $\left(150 \times 150 \mathrm{~nm}^{2}\right)$ : Jeder Rasterpunkt der Karte entspricht einer eigenen STS-Messkurve. Ihre minimale Steigung (Leitwert) nahe $\boldsymbol{\theta} V$ ist als farbiger Messpunkt in der Leitwertmap eingetragen.

b) $\chi^{2}-$ Abweichung des Kennlinienfits als Fehlerbetrachtung

Um die Qualität der einzelnen Kennlinienfits beurteilen zu können, wird entsprechend der $\chi^{2}-$ Minimierung:

$$
\chi^{2}\left(p_{1}, p_{2}, \ldots\right)=\frac{1}{n^{e f f}-p} \sum_{i}\left[y_{i}-f\left(x_{i}, p_{1}, p_{2}, . .\right)\right]^{2}
$$


eine weitere Analyse durchgeführt. Die in Abbildung 4.4 (b) gezeigte graphische Darstellung gibt Aufschluss über vorhandene Abweichungen. Die Variablen $p_{x}$ stellen dabei die Gewichtung der Freiheitsgrade, $n^{e f f}$ die Anzahl der Messpunkte je Kennlinie und $p$ die Anzahl der Parameter dar. Durch die Summe wird die Gewichtung der Messpunkte betrachtet. Anhand des Beispiels in Abbildung 4.4 lässt sich erkennen, dass hier kein zusammenhängender Bereich einen größeren Auswertungsfehler aufweist. Generell liegt eine geringe konstante Abweichung vor, die im Mittel bei $\chi^{2}=1,0 \cdot 10^{-3} n A^{2}$ liegt.
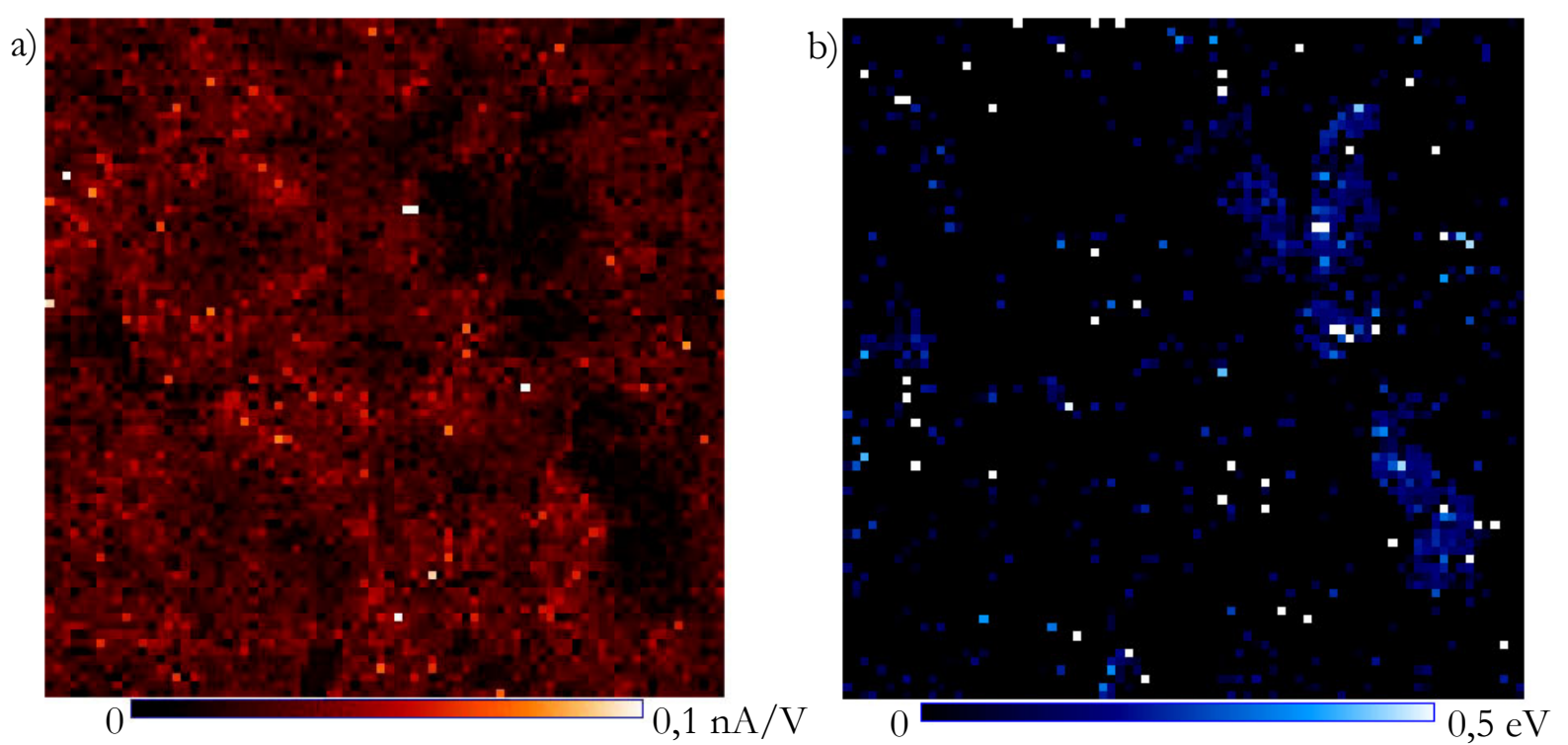

Abbildung 4.5: a) zweidimensionale Leitwertkarte, die aus den Steigungen des linearen Fit der Kennlinien um $0 \mathrm{~V}$ errechnet wurde

b) Darstellung der Gapbereiche - Aufgetragen ist die Breite des Gaps

Als zusätzliche Verifikation dieser Ergebnisse werden die Kennlinien im Bereich nahe $0 \mathrm{~V}$ linear gefittet. Dies geschieht durch die Vorgabe des Analysebereichs durch eine fest definierte Anzahl an Messpunkten. Energie - Intervalle von ca. \pm 0,2 eV um Null werden gewählt, da sich in dieser Größenordnung die Bandlücke (Gap) bei Manganaten befindet [65; 66]. Die Leitfähigkeit wird, wie beschrieben, aus der Steigung berechnet und ist als Leitwertkarte in Abbildung 4.5 (a) dargestellt. Die erkennbare Struktur gleicht der in Abbildung 4.4 (a). In beiden sind in der rechten Hälfte zwei schlechter leitfähige bzw. isolierende Flächen zu erkennen. Von Interesse ist nun die Gap - Breite (Größe der Energielücke). Hierzu werden die Ableitungen (Abbildung 4.3 (c2)) betrachtet und ihre Schnittpunkte mit der x - Achse bestimmt. Hieraus lässt sich die vorliegende Energielücke bestimmen und wiederum in einen Farbwert kodieren. Das Ergebnis dieser Auftragung ist in Abbildung 4.5 (b) dargestellt. Deutlich ist zu erkennen, dass die in der zweidimensionalen Energielückendarstellung blau dargestellten Regionen in der Leitwertmap 
schwarz, also isolierend sind. Die Kombination aus beiden Bildern ergibt eine Karte, die sowohl die Leitfähigkeit der Probe als auch die Energielücke an verschiedenen Orten darstellen kann.

\subsection{Simulation von Widerstandsnetzwerken}

Im Kapitel 4.1 wurden Übersichtskarten vorgestellt, die ortsaufgelöst Leitfähigkeiten sichtbar machen. Generell ist von Interesse, ob anhand der zweidimensionalen Darstellung der STSMessungen, die Bereiche der Manganatoberfläche als metallisch leitfähig oder isolierend klassifizieren, auch ein Vergleich zu dreidimensionalen Transportmessungen ${ }^{11}$ möglich ist. An dieser Stelle sei erwähnt, dass sich STS - und Transportmessungen prinzipiell unterscheiden: STS betrachtet die elektronische Zustandsdichte an der Oberfläche und kann somit die punktuelle Tunnelleitfähigkeit beschreiben. Der Tunneleffekt ist im Gegensatz zum Transportwiderstand im Wesentlichen temperaturunabhängig [67]. Bei Transportmessungen verlaufen die Ströme dreidimensional innerhalb der Probe. Sie besitzen eine hohe Temperaturabhängigkeit, können aber keinen Aufschluss über lokale Leitfähigkeiten geben. Geht man von einer elektronischen Phasenseparation aus, entstehen leitfähige und isolierende Domänen. Bei tiefen Temperaturen liegt hauptsächlich metallisches Verhalten vor. Wird die Temperatur nun erhöht, wachsen die halbleitenden Domänen auf Kosten der metallischen Bereiche. Schließlich existieren nur noch wenige geschlossene Strompfade, die beim Übergang ebenfalls unterbrochen werden - mit anderen Worten liegt ein perkolatives Verhalten vor. Der Vergleich zwischen Transportmessungen und STS-Leitwertkarten kann jedoch nicht direkt erfolgen, sondern erfordert eine geeignete Auswertung der Leitwertkarten.

Abbildung 4.6 (a) zeigt, wie sich das Raster von Leitwerten an der Oberfläche darstellt. Hierbei wird davon ausgegangen, dass sich die einzelnen Messpunkte wie Felder eines Schachbrettes anordnen. Um nun einen Eindruck davon zu erhalten, wie Ströme in diesem zweidimensionalen Gitter von einem Punkt zum anderen fließen, muss das Gitter geeignet in ein Netzwerk von Widerständen R bzw. Leitfähigkeiten $\sigma$ umgesetzt werden $(\sigma=1 / R)^{12}$. Aufgrund der Gesetze für Reihen- und Parallelschaltungen von Widerständen und Leitwerten ist es im Folgenden angebracht, Leitwerte zu betrachten. Der Gesamtleitwert einer Parallelschaltung ergibt sich als

\footnotetext{
11 Hierbei werden über elektrische Kontakte an der Probenoberfläche die Leitungseigenschaften der Probe untersucht. Um den Widerstand der Zuleitungen vernachlässigen zu können wird häufig die so genannte Vier-PolMessung angewandt.

$12 \mathrm{Im}$ Folgenden wird generell von der Bildung eines diskreten Rasters ausgegangen. Eine Betrachtung der Geometrie der Oberfläche entfällt, da bei der STS-Messung nur punktuell der Tunnelleitwert bestimmt wird. Somit entfällt hier die Bestimmung des geometrieabhängigen spezifischen Widerstands $\rho$.
} 
Summe der Leitwerte; bei einer Reihenschaltung addieren sich die Kehrwerte zum Kehrwert des Gesamtleitwertes entsprechend der Kirchhoffschen Gesetze. Möchte man nun den Gesamtwiderstand einer Leitwertkarte bestimmen, erkennt man schnell, dass dieses Problem nicht einfach zu lösen ist (Abbildung 4.6). Daher betrachten wir einen einzelnen Rasterpunkt und dessen Leitwert $\sigma_{1}$ (Abbildung 4.7).

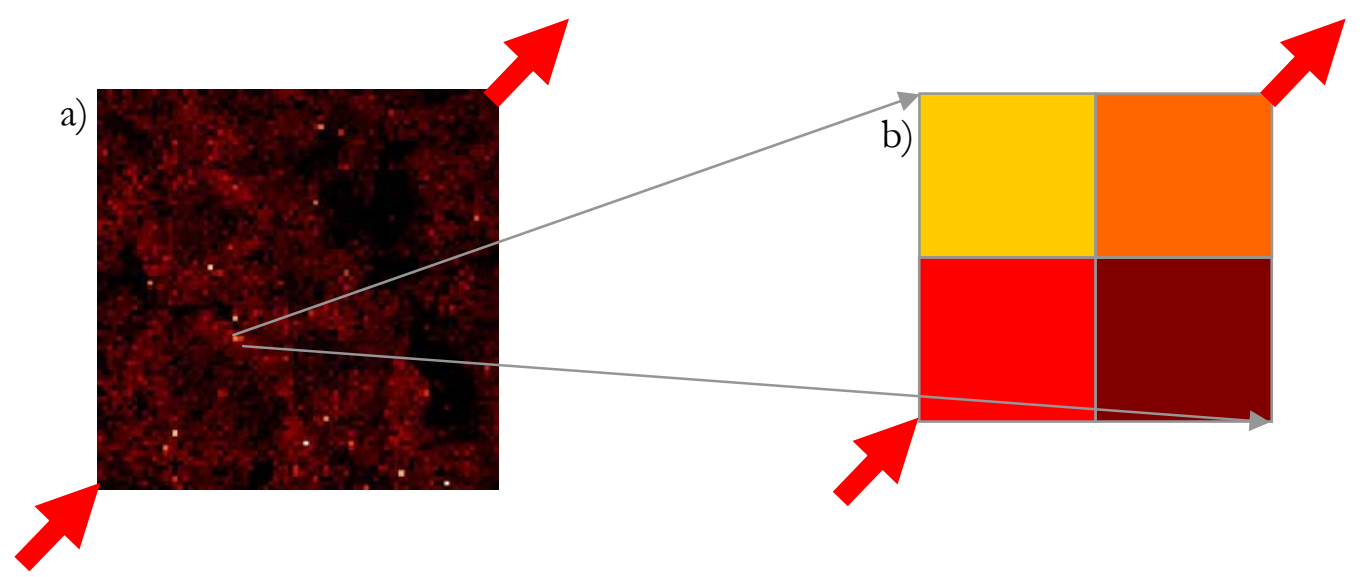

Abbildung 4.6: a) Anhand der Leitwertkarte aus Abbildung 4.4 soll ein diagonal verlaufender Stromfluss simuliert werden (durch die roten Pfeile symbolisiert). Das System erscheint relativ komplex und auch eine Reduktion auf vier Widerstände (b) vereinfacht das Problem nicht.

Jedes Feld kann sowohl auf diagonalem wie auch vertikalem oder horizontalem Wege von Strom durchflossen werden. Um diesem gerecht zu werden, wird jeder Rasterpunkt mit Kontaktlinien versehen, die diese Stromflüsse zulassen. Dies führt zu einem Ersatz-Netzwerk von acht Einzelwiderständen, wie sie in Abbildung 4.7 gezeigt sind.

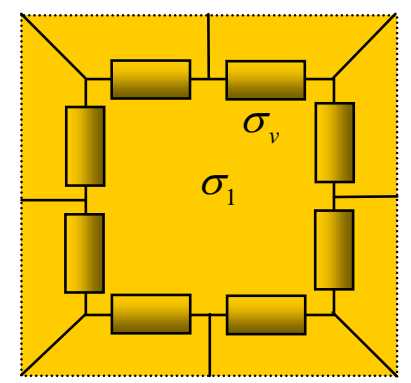

Abbildung 4.7: Entstehung des Ersatzschaltbildes für einen Rasterpunkt: Die Kontakt- bzw. Stromflussmöglichkeiten werden eingezeichnet. Schließlich werden die internen Ersatzleitwerte $\sigma_{v}$ eingefügt. Jeder (virtuelle) Ersatzleitwert ist genau von doppeltem Wert wie der Leitwert $\sigma_{1}$ des Rasterpunktes. 
Aus Symmetriegründen müssen die virtuellen Verbindungsleitwerte $\sigma_{v}$ zwischen diesen Kontaktpunkten miteinander identisch sein. Da der Gesamtleitwert eines Rasterpunktes bekannt ist, kann der Wert des virtuellen Leitwertes berechnet werden als:

$$
\sigma_{v}=2 \sigma_{1}
$$

Diese Ersatzschaltung für einen Messpunkt kann nun auf das gesamte Raster übertragen werden. Hierzu betrachtet man z.B. einen Ausschnitt entsprechend Abbildung 4.6 b) der Größe $2 * 2$ Messpunkte. Da alle internen Leitwerte eines Rasterpunktes identisch sind, können die Verbindungslinien senkrecht zu den Seitenkanten eines Feldes (blau dargestellt) vernachlässigt werden. Hier entsteht keine Potentialdifferenz, d.h. es werden über diese Kontakte keine Ströme fließen (Potentialdifferenz $=0$ ).

a)

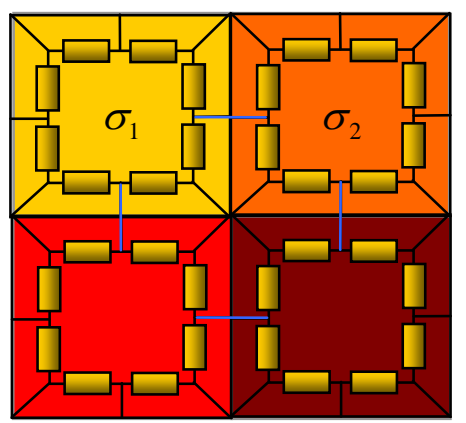

b)

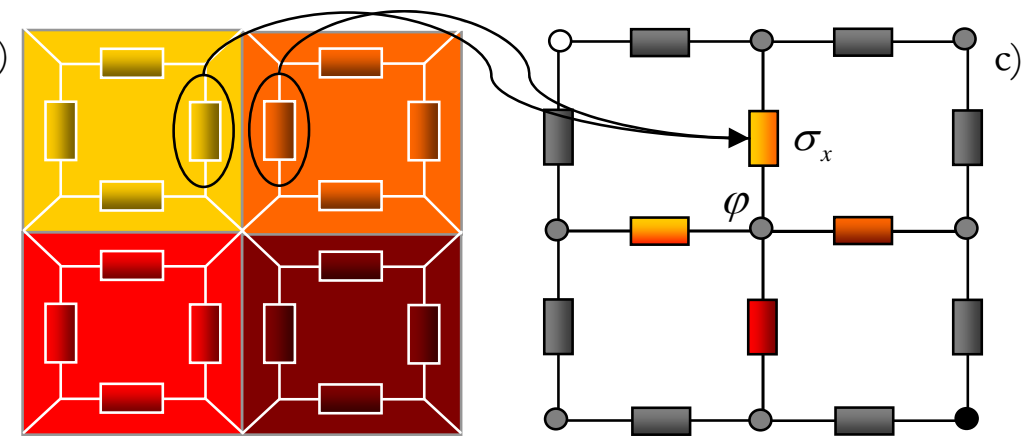

Abbildung 4.8: In Darstellung (a) ist die Kombination aus Abbildung 4.6 (a) und Abbildung 4.7 zusammengefasst. Der Stromfluss ist in alle Richtungen möglich. Eine Vereinfachung (b) ergibt sich, da die Verbindungslinien senkrecht zu den Seitenkanten eines Feldes vernachlässigt werden. Die gezeichneten Leitwerte sind vom gleichen Betrag wie der Leitwert des Rasterpunktes. Parallel verlaufende Leitwerte können zusammengefasst werden und ergeben die Schaltung (c)

Die nun in Reihe geschalteten Leitwerte können durch ihre Kehrwerte zusammengerechnet werden (Abbildung 4.8 b)). Dann hat jeder der vier internen Leitwerten genau den Betrag des Leitwertes des Rasterpunktes, z.B. $\sigma_{1}$. Nach den genannten Regeln für Parallelschaltungen können nun die Leitwerte entlang der Kanten zusammengefasst werden; es entsteht also ein Leitwertnetzwerk, dessen einzelne Leitwerte genau der Summe der Leitwerte ihrer benachbarten Felder entsprechen:

$$
\sigma_{x}=\sigma_{1}+\sigma_{2}
$$


Aus einer beliebigen Anzahl von Messpunkten kann so ein Netzwerk aus Leitwerten erstellt werden.

Zur Simulation von Transportmessungen muss nun berechnet werden, wie sich bei Anlegen einer Spannung zwischen zwei Punkten des Netzwerkes der Stromfluss aufbaut. Da die Verknüpfung des Netzwerkes keine analytische Auswertung auf einfachem Wege erlaubt, wird der Gesamtwiderstand aus der gesamten Leistung des Netzwerkes errechnet:

$$
R_{\text {ges }}=\frac{U_{\text {ges }}^{2}}{P_{\text {ges }}}
$$

Bei den Kontaktpunkten, über denen die Spannung $U_{g e s}$ abfällt, handelt es sich vorzugsweise, (aber nicht notwendigerweise) um gegenüberliegende Eckpunkte des Netzes (s. unten). Für die endgültige Berechnung ist es allerdings notwendig die ohmsche Leistung $P_{g e s}$ zu berechnen, die im gesamten Netzwerk anfällt. Hierbei gilt:

$$
P_{g e s}=\sum P_{i}
$$

wobei mit $P_{i}$ sämtliche Leistungen gemeint sind die an den Leitwerten des Netzwerkes anfallen. Diese Einzelleistungen lassen sich leicht berechnen, wenn davon ausgegangen wird, dass jedem Kreuzungspunkt der Vernetzung (Abbildung $4.8 \mathrm{c}$ )) ein Potential $\varphi_{i}$ zugeordnet werden kann. Die Spannung $U$, die über einem Leitwert $\sigma$ (bzw. Widerstand $R$ ) abfällt, kann dann als Differenz der beiden angrenzenden Potentiale berechnet werden:

$$
U=\Delta \varphi=\varphi_{i+1}-\varphi_{i}
$$

Hieraus errechnet sich dann der Stromfluss und die ohmsche Leistung am Leitwert zu

$$
\begin{gathered}
I=\frac{U}{R}=U \cdot \sigma \\
P=U \cdot I=\sigma \cdot\left(\varphi_{i+1}-\varphi_{i}\right)^{2}
\end{gathered}
$$

Es ist also notwendig, sämtliche Potentiale an den Kreuzungspunkten zu kennen, um den Gesamtwiderstand der Anordnung zu errechnen. Zunächst wird die gesamte Anordnung in sich 
potentialdifferenzfrei gesetzt, d.h. alle Potentiale seien gleich $0.5^{(13)}$. Die von außen angelegte Spannung $U_{g e s}$ wird nun dadurch realisiert, dass die Kontaktpunkte (Abbildung 4.6 rote Pfeile) ein Potential von $\varphi=1$ bzw. $\varphi=0$ erhalten ${ }^{14}$. Durch die Wahl der inneren Potentiale zu 0.5 wird die effektiv nötige Rechenzeit erheblich reduziert.

Die einzelnen Potentialwerte werden nun iterativ berechnet. Hierbei kann in beliebiger Reihenfolge vorgegangen werden, da alle Potentiale auf Basis der alten Potentialkarte berechnet werden und erst anschließend alle neuen Werte in die Potentialkarte eingetragen werden. Die eigentliche Berechnung folgt streng den Kirchhoffschen Regeln (Abbildung 4.9 (a)).

a)

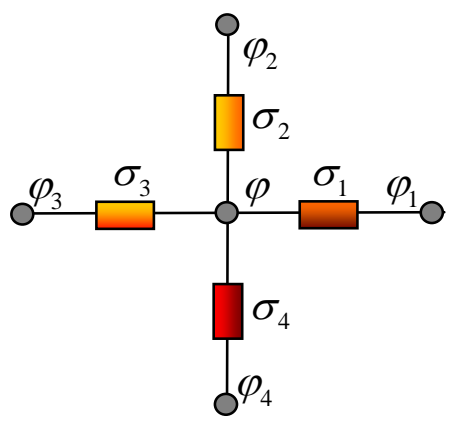

b)

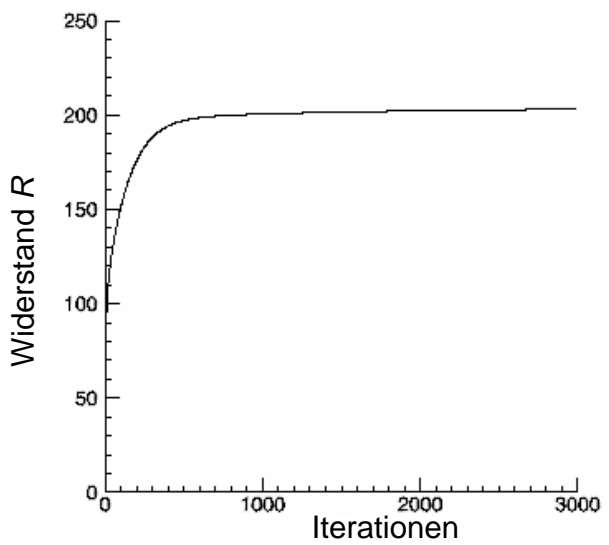

Abbildung 4.9: a) Potentialberechnung an einem Knotenpunkt durch Einsatz der Kirchhoffschen Regeln

b) Widerstandsentwicklung bei dem Durchlaufen von 3000 Iterationen - ein stationärer Zustand hat sich bei 203 eingestellt

Das Potential $\varphi$ wird so bestimmt, dass die Summe aller Ströme im zugehörigen Knotenpunkt verschwindet, also nach Gleichung (4.7):

$$
\begin{gathered}
\sum I_{i}=\sum \sigma_{i}\left(\varphi_{i}-\varphi\right)=0 \\
\Leftrightarrow \varphi=\frac{\sum \sigma_{i} \varphi_{i}}{\sum \sigma_{i}}
\end{gathered}
$$

Auf diese Weise werden sämtliche Knotenpunkte abgerastert. Die als Kontakt für die von außen angelegte Spannung dienenden Punkte werden jedoch auf dem ursprünglichen Wert belassen, entsprechend einer stabilen Spannungsquelle. Der stationäre Zustand ist erreicht, sobald ein stabiler Gesamtwiderstandswert erreicht wurde, die Iteration wird dann abgebrochen (Abbildung

\footnotetext{
${ }^{13}$ Dieser Wert ist willkürlich. Lediglich Potentialdifferenzen sind für die Auswertung von Bedeutung.

${ }^{14}$ Auch dieser Wert ist willkürlich, er sorgt lediglich dafür, dass $U_{g e s}=1$ ist.
} 
4.9 (b)). Aufgrund der Rechenweise ist auch ersichtlich, warum es von Vorteil ist, die Anfangspotentiale für die nicht kontaktierten Punkte auf den Mittelwert der Eckpotentiale zu setzen: Der Stromfluss dringt so von beiden Seiten in das Netzwerk ein, der stationäre Zustand wird schneller erreicht.

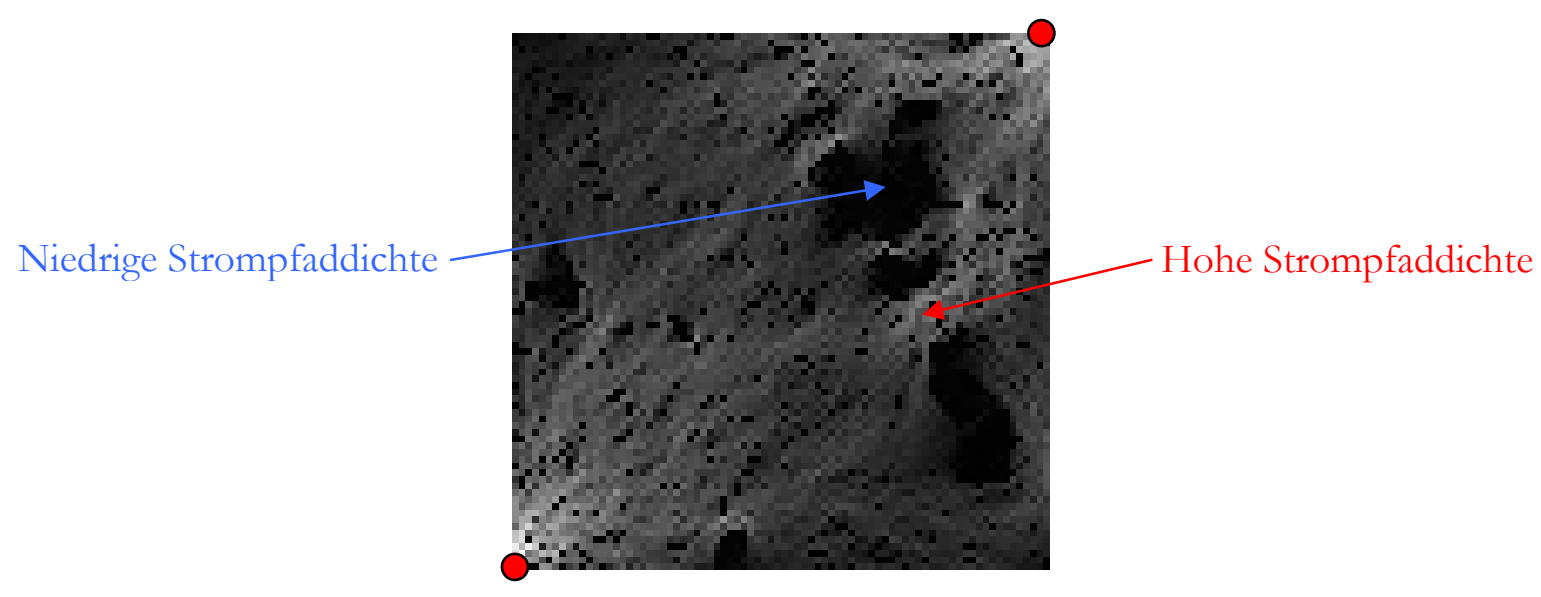

Abbildung 4.10: Stromverlaufskarte von LCMO (entsprechend der Leitwertmap aus Abbildung 4.4 a). Der Stromfluss ist zwischen den beiden rot eingezeichneten „Kontakten“ simuliert worden. Bereiche mit hoher Strompfaddichte sind hell dargestellt. Es ist deutlich zu erkennen, dass Bereiche mit niedrigem Leitwert eine geringe Strompfaddichte vorweisen. Dem hingegen sind metallisch leitfähige Bereiche in diesem Fall homogen durchsetzt.

Die Auswertung der simulierten Netzwerke erfolgt auf verschiedene Weisen. Neben der Bestimmung des Transportwiderstandes (bzw. -leitwertes) können z.B. Stromflusskarten oder Leistungskarten erstellt werden. Auf ersteren kann gezeigt werden, wo sich Strompfade ausbilden, aber auch wie Strom in eine Probe eindringt. Für das gewählte Beispiel ist das Ergebnis in Abbildung 4.10 dargestellt.

Für Proben mit wenig zusammenhängenden leitfähigen Bereichen ist es hierbei sinnvoll, die Kontakte nicht nur mit einem Punkt festzulegen, sondern Bereiche (z.B. einen Eckbereich von $3 \times 3$ Knotenpunkten) auf festes Potential $\mathrm{zu}$ legen. So wird vermieden, dass der Gesamtwiderstand zu stark von den Eckleitwerten bestimmt wird. 


\section{Ergebnisse und Auswertungen}

In diesem Kapitel sollen topographische und spektroskopische Messungen an Manganaten vorgestellt werden. Hierzu kommt das in Kapitel 3 vorgestellte VT-STM zum Einsatz. Im ersten Kapitel wird die Austrittsarbeit beispielhaft bestimmt. Hierdurch ergibt sich der maximale Wertebereich für die Tunnelspannung, in dem Messungen durchgeführt werden können. In den folgenden zwei Kapiteln werden Messungen an LCMO und LSMO bei fester, tiefer Temperatur vorgestellt. Temperaturabhängige Messreihen folgen in den letzten drei Paragraphen und werden mit Transportmessungen verglichen.

Alle vorgestellten Messungen wurden unter UHV - Bedingungen durchgeführt. Nach Einschleusen in die UHV - Kammer wurden die zu untersuchenden Proben auf unter $50 \mathrm{~K}$ abgekühlt um Sauerstoffdegradation an der Oberfläche durch Desorption zu vermeiden. Sauerstoffverlust führt $\mathrm{zu}$ Widerstandsänderungen der Probe und somit $\mathrm{zu}$ nicht reproduzierbaren STM - und STS - Messungen. Näheres hierzu ist im Anhang in Kapitel $8.1 \mathrm{zu}$ finden. Als Sondenmaterial wurde bei allen folgenden Messungen PtIr eingesetzt, da Wolframspitzen bei zahlreichen Versuchen an Manganatfilmen nur eine mäßige Qualität der Messungen aufwiesen.

\subsection{Bestimmung der Austrittsarbeit}

Das Prinzip der Rastertunnelmikroskopie wurde in den Kapiteln 3 und 3.2 beschrieben. Es ist jedoch wichtig, die lokale Barrierenhöhe zu kennen und diese keinesfalls zu überschreiten. Ansonsten überwinden die Elektronen die Vakuumbarriere direkt und es kommt zur Feldemission. Die so durchgeführte Messung zeigt ein nicht erwartetes Messergebnis [68]. Als Austrittsarbeit wird die Arbeit bezeichnet, die benötigt wird, um ein Leitungselektron des Festkörpers von seiner Oberfläche zu entfernen. Sie ist primär abhängig von der lokalen Barrierenhöhe $\Phi$. Gemäß Gleichung (3.1) folgt unmittelbar:

$$
\frac{d \ln I}{d s} \propto-2 \sqrt{\Phi}
$$

Zur Bestimmung der maximal einzustellenden Tunnelspannung wird nach Ausschalten des Regelkreises der fließende Tunnelstrom in Abhängigkeit zum Abstand $s$ gemessen. Anschließend 
wird der Strom logarithmisch gegen den Abstand aufgetragen. Die Austrittsarbeit läst sich aus der Geradensteigung des Graphen bestimmen.

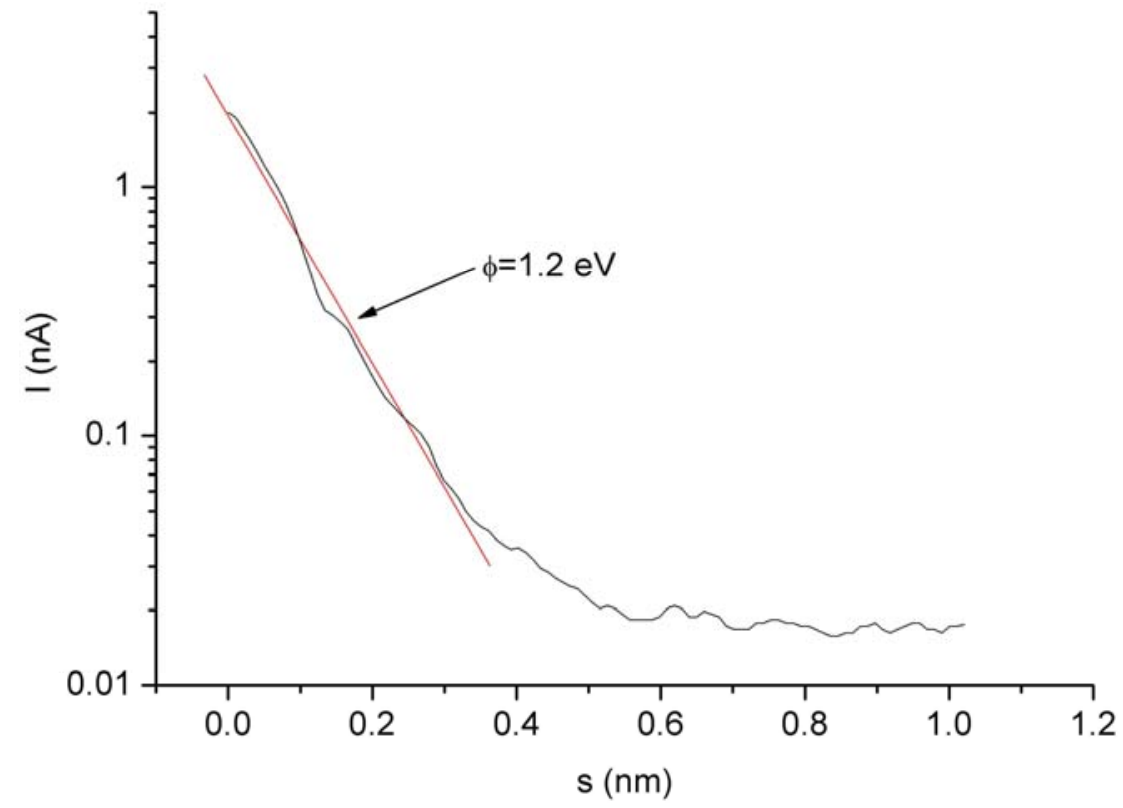

Abbildung 5.1: Logarithmische Auftragung des Tunnelstroms gegen den Abstand der Spitze. Aus der linearen Steigung im Anfangsbereich lässt sich die Austrittsarbeit bestimmen.

Die in Abbildung 5.1 dargestellte Strom - Abstands - Kennlinie, verläuft in der halblogarithmischen Darstellung anfangs linear. Dies deutet an, dass ein Tunnelprozess stattgefunden hat. Die Messungen wurden mehrfach an verschiedenen Proben durchgeführt. Abbildung 5.1 zeigt ein typisches Beispiel gemessen an $\mathrm{L}_{0.7} \mathrm{Sr}_{0.3} \mathrm{MnO}_{3}$ bei $\mathrm{T}=30 \mathrm{~K}$. Die bestimmte Austrittsarbeit liegt bei $\Phi=1.2 \mathrm{eV}^{15}$, also deutlich niedriger als bei vielen Metallen. Bei Gold bestimmt sich der Wert z.B. zu $\Phi=4.8 \mathrm{eV}$ und bei Wolfram zu $\Phi=4.5 \mathrm{eV}$ [69]. Dieses Ergebnis zeigt, dass die maximale Tunnelspannung den Wert von $U_{T}=1,2 \mathrm{~V}$ nicht überschreiten darf. Daher wird bei den folgenden STM - und STS - Messungen meist eine Spannung von $U_{T}=$ $0.8 \mathrm{~V}$ oder weniger genutzt um entstehende Bildartefakte zu vermeiden.

\footnotetext{
15 Andere Oxyde wie z.B. $\mathrm{Nb}_{2} \mathrm{O}_{5}(\Phi=1.0 \mathrm{eV})$ zeigen ähnliche Werte. Wird ein Diamant mit Stickstoff dotiert, verändert sich seine Austrittsarbeit von $\Phi=2.6 \mathrm{eV}$ zu $\Phi=1.5 \mathrm{eV}$. aus [70]
} 


\section{$5.2 \mathrm{LCMO}$ auf $\mathrm{MgO}$ bei $78 \mathrm{~K}$}
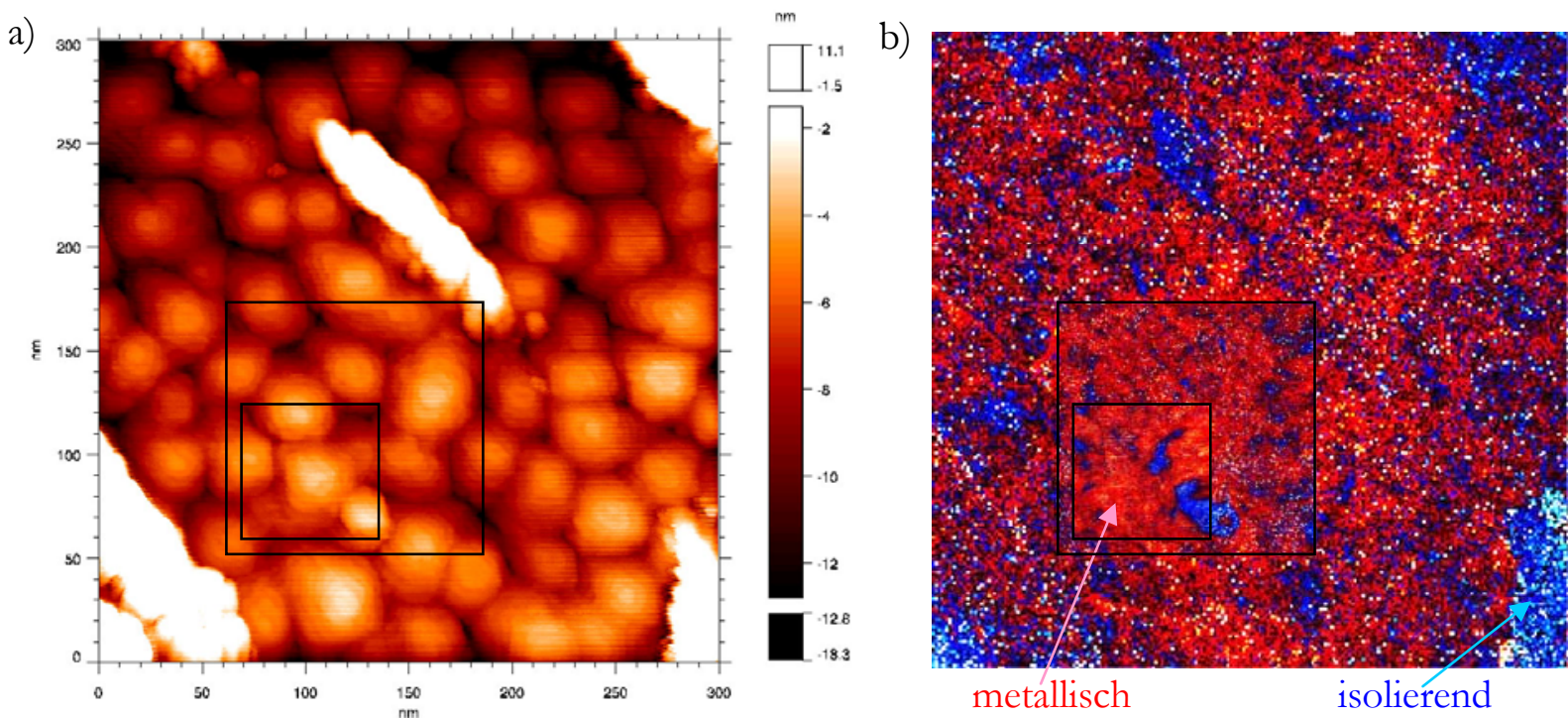

Abbildung 5.2: (a) zeigt die Topographie von LCMO auf MgO (Scanbereich: $300 \mathrm{~nm} \times 300 \mathrm{~nm}$; Raster: $1200 \times 1200)$. Zwei weiter durchgeführte Messungen werden durch schwarze Quadrate angedeutet. Bei jedem fünften Rasterpunkt in $x$ - und $y$-Richtung sind Kennlinien aufgezeichnet worden. Die Kombination von Gap- und Leitwertkarten (entsprechend Kapitel 4.1) ist in (b) dargestellt: rot sind metallisch leitfähige und blau isolierende Bereiche dargestellt. Die Ergebnisse der beiden Nahaufnahmen sind hier ebenfalls implementiert.

In Abbildung 5.2 sind Ergebnisse von STM - und STS - Messungen an einem $50 \mathrm{~nm}$ dicken $\mathrm{La}_{0.7} \mathrm{Ca}_{0.3} \mathrm{MnO}_{3}$ - Film dargestellt. Es wird ein $\mathrm{MgO}$ - Substrat verwendet, um epitaktisches Kornwachstum zu gewährleisten [71]. Die Schichtherstellung erfolgte durch die MAD Technik $^{16}$. Zur Aufnahme der Topographie wurde eine Tunnelspannung von $U_{T}=0.8 \mathrm{~V}$ bei einem Tunnelstrom von $I_{T}=0.6 \mathrm{nA}$ gewählt. Das Bild in (a) besteht aus 1200 Zeilen und Spalten und bildet einen Bereich von $(300 \mathrm{~nm})^{2}$ ab. Deutlich ist eine gleichmäßige Kornstruktur mit einem mittleren Korndurchmesser von $36 \mathrm{~nm}$ zu erkennen, die zu einer RMS ${ }^{17}$ - Rauhigkeit von 0,93 nm führt. Die Kornstruktur lässt Parallelen zu Oberflächenuntersuchungen an $\mathrm{YBaCuO}-$ Schichten erkennen [72]. Es sind, wenn auch nur schwach ausgeprägt, Wachstums - Spiralen erkennbar (siehe Abbildung 5.4). Parallel zu den topographischen Informationen wurden Kennlinien ${ }^{18}$ mit einem Raster von $240 \times 240$ Punkten aufgezeichnet und entsprechend der in Kapitel 4.1 beschriebenen Analysemethode ausgewertet. Dargestellt wird das Ergebnis in (b) und ist mit dem Oberflächenbereich aus (a) identisch. Kennlinien mit metallischem Charakter sind rot

\footnotetext{
${ }^{16}$ Die LCMO-Schicht wurde von V. Moshnyaga, I. Physikalisches Institut, Universität Göttingen präpariert

${ }^{17}$ RMS: root mean square

${ }^{18}$ Bereich: 200 Messwerte von $-0.5 \mathrm{~V}$ bis $0.5 \mathrm{~V}$; Aufzeichnungsdauer pro Wert: $2 \cdot 10^{-4} \mathrm{sec}$
} 
dargestellt. Wurde bei der Analyse eine Bandlücke festgestellt, so ist die Breite der Bandlücke als blauer Farbton aufgetragen. Zusätzlich zur Übersichtsmessung wurden mit den gleichen Parametern weitere Messungen mit $118 \mathrm{~nm}$ und $65 \mathrm{~nm}$ Kantenlänge durchgeführt. Schematisch sind die beiden Bereiche in Abbildung (a) und (b) als schwarze Quadrate eingezeichnet. Die Ergebnisse der Kennlinienanalyse sind in (b) ortsgenau implementiert. Erst durch die höhere Auflösung kann zwischen halbleitend isolierenden und metallischen Bereichen unterschieden werden. Domänen mit isolierendem Charakter grenzen sich deutlich von der übrigen, metallischen Oberfläche ab.

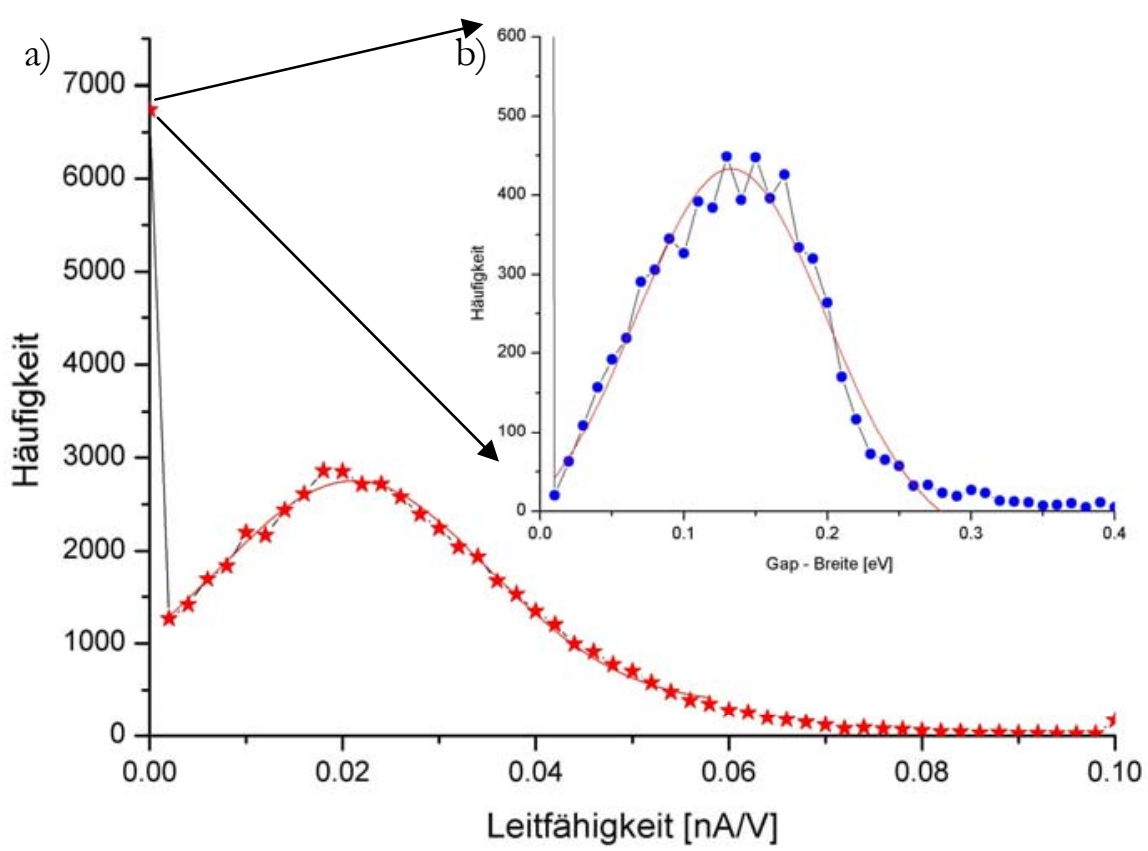

Abbildung 5.3: Häufigkeitsverteilungen der Leitfähigkeiten in (a) und der Bandlücke in (b) für die Messung mit höchster Auflösung (65 nm Kantenlänge). Die jeweils durchgezogene rote Kurve entstammt einer gefitteten Gaußunktion zur Maximumsbestimmung.

Die Ergebnisse der Messung mit höchster Vergrößerung sind in Abbildung 5.3 und Abbildung 5.4 noch einmal separat dargestellt. Die Graphen aus Abbildung 5.3 zeigen Histogramme für die Leitfähigkeiten (a) und die Gapbreite (b). Erkennbar ist, dass aufgrund der niedrigen Temperatur von $T=78 \mathrm{~K}$ die Probe größtenteils eine sehr gute Tunnelleitfähigkeit mit einem mittleren Leitwert von $2,5 \cdot 10^{-2} \mathrm{nA} / \mathrm{V}$ aufweist und nur recht wenige Kennlinien (ca. $12 \%$ ) isolierendes Verhalten zeigen. Ersichtlich wird dies aus der Leitfähigkeitsverteilung: Sie weist ein breites Maximum bei ca. $2 \cdot 10^{-2} n A / V$ und einen recht scharfen Peak bei $0 n A / V$ auf. Die Kennlinien, die summiert diesen Peak ergeben, zeigen kein leitfähiges Verhalten. Sie unterscheiden sich jedoch in den Breiten der gemessenen Bandlücken, die in b) als weiteres Histogramm aufgetragen sind. Betrachtet man beide als gaußverteilt, kann durch die Wahl passender Parameter das 
jeweilige Maximum bestimmt werden. Für die Leitfähigkeit ergibt sich dieses zu $2.1 \cdot 10^{-2} \mathrm{nA} / \mathrm{V}$ und die mittlere Gapbreite liegt bei $0.13 \mathrm{eV}$.

a)

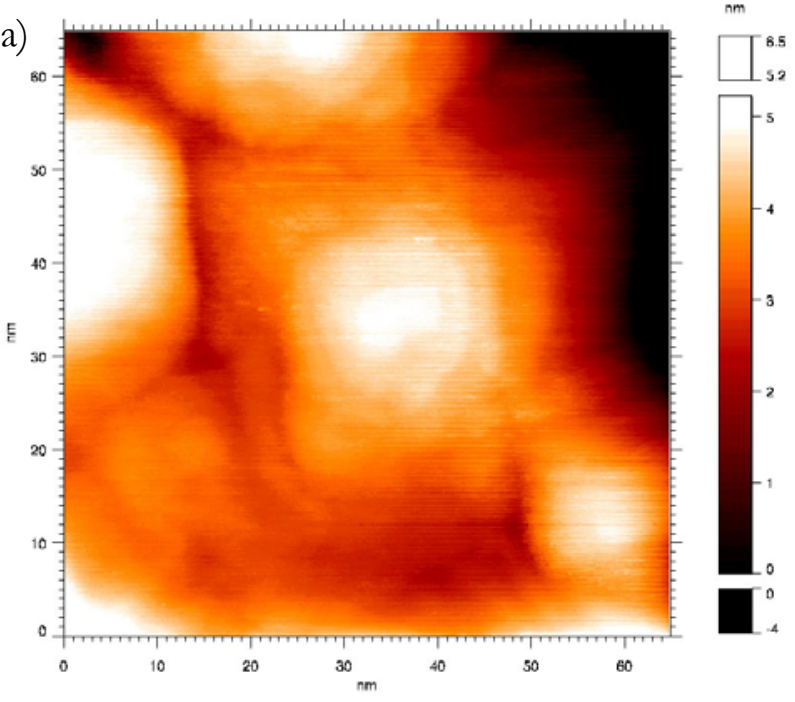

b)

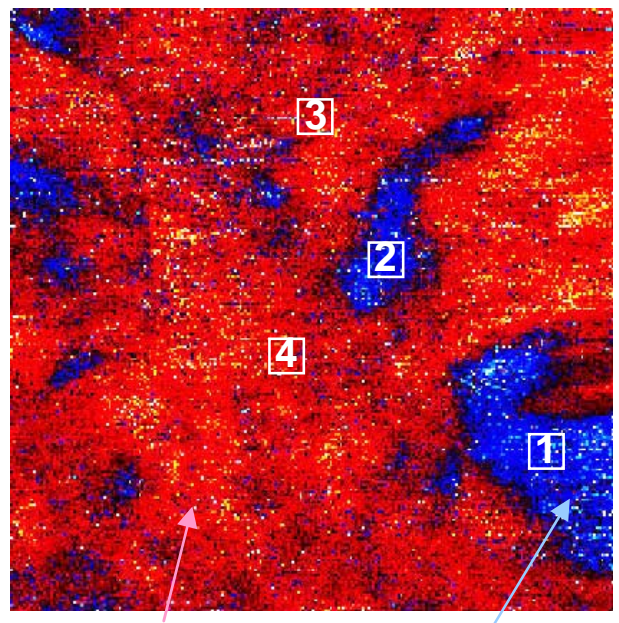

metallisch

isolierend

c)

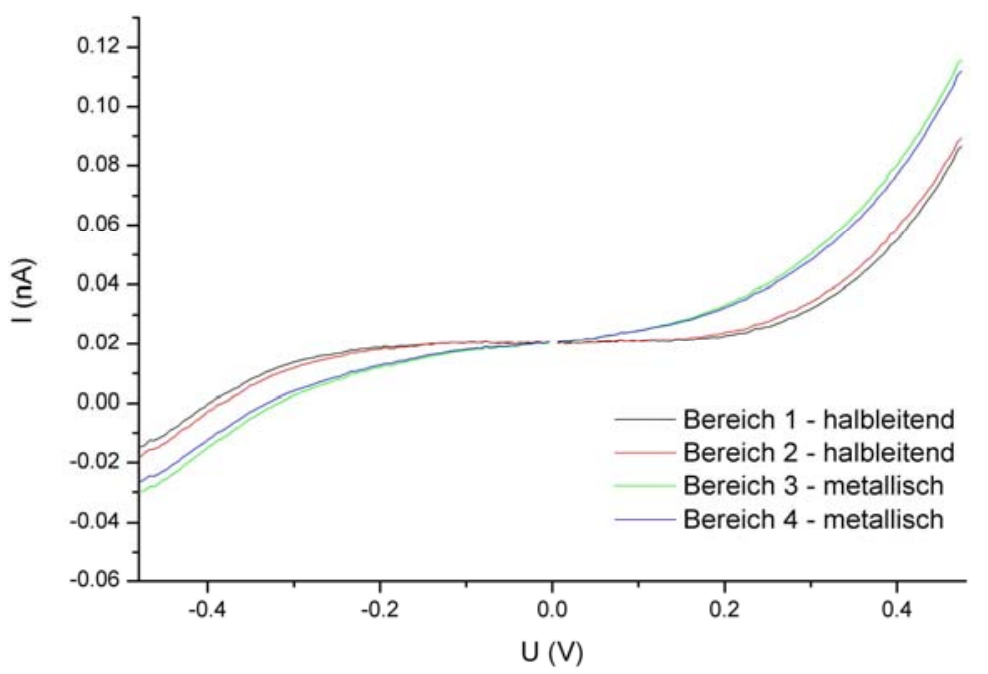

Abbildung 5.4: In (a) ist die Nahaufnahme aus Abbildung 5.2 dargestellt. (b) stellt die Kombination aus Leitwert- und Gapverteilung dar. Es sind homogene Domänen zu erkennen. Eine Korrelation mit der Topographie kann nicht nachgewiesen werden. Korn und Korngrenze können nicht eindeutig einem metallisch oder isolierendem Verhalten zugewiesen werden. Vielmehr ist zu erkennen, dass isolierende Domänen tendenziell auch innerhalb von Körnern auftreten können. Einige Kennlinien sind exemplarisch in (c) dargestellt. Sie stammen aus den durch Nummern gekennzeichneten Bereichen in (b). Deutlich ist der Unterschied in den Steigungen der isolierenden und leitfähigen Bereiche zu erkennen.

Das STM - Bild in Abbildung 5.4 (a) zeigt ein zentrales Korn mit einem Durchmesser von ca. $45 \mathrm{~nm}$. Parallel hierzu befindet sich in (b) die Leitfähigkeitsverteilung für den Bildausschnitt in (a). Die scharfe Trennung von leitfähigen und isolierenden Domänen ist ersichtlich. Zusätzlich zeigt sich, dass die elektronische Domänenstruktur nicht von der topographischen Kornstruktur 
abhängt. Das zentrale Korn besteht nicht aus einer einheitlichen Domäne, es existieren von einander getrennte metallische und isolierende Bereiche innerhalb eines Korns. Ein Ergebnis, das auf intrinsische Effekte hindeutet.

Über die in Abbildung 5.4 (b) mit 1 bis 4 gekennzeichneten Bereiche mit einer Kantenlänge von je 12 Messpunkten wurden die darin enthaltenen Kennlinien gemittelt und in c) aufgetragen. Kennlinien aus den Bereichen eins und zwei verlaufen um $0 \mathrm{~V}$ flach ohne Steigung und weisen eine Bandlücke mit der Gapbreite von ca. 0,15 eV auf. Deutlich unterscheiden sich die beiden Kennlinienverläufe aus den anderen beiden Bereichen, sie zeigen nahe $O V$ eindeutig metallischen Charakter.

Die Leitwerte aus Abbildung 5.4 (b) sind mit Hilfe der in Kapitel 4.2 beschriebenen Widerstandsnetzwerksimulation analysiert worden. In Abbildung 5.5 ist die daraus entstandene Karte mit den nach Kapitel 4.2 bestimmten Stromdichten gezeigt. Helle Bereiche weisen eine höhere Dichte als dunkel dargestellte auf. Sie zeigt eine gute Übereinstimmung mit der vorgegebenen Leitwertkarte. Bei schlecht leitenden Stellen der Probe ist die resultierende Stromdichte gering oder nicht vorhanden. Dem gegenüber besitzen leitfähige Areale eine hohe Dichte. Diese zeigt sich zudem als sehr homogen verteilt, ein Indiz für das Vorliegen der Phasenseparation.

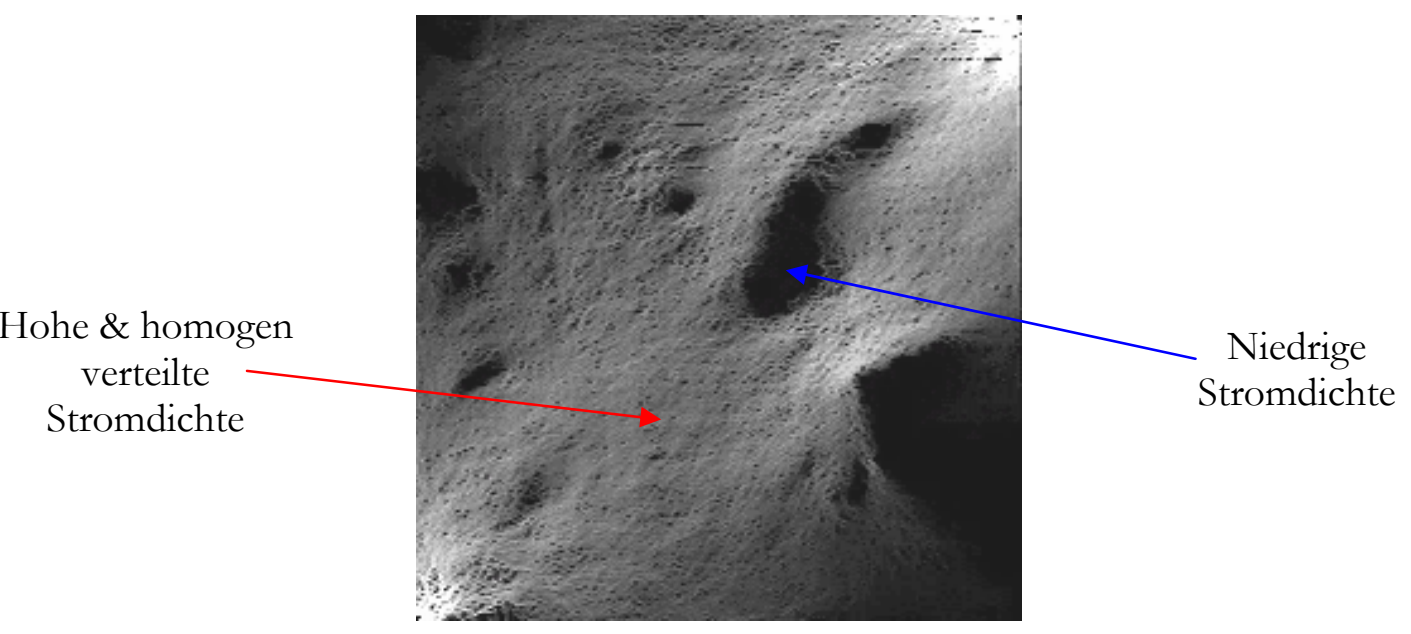

Abbildung 5.5: Simulierte Stromdichte basierend auf den Ergebnissen aus Abbildung 5.4 (b). Helle (dunkle) Bereiche weisen eine hohe (niedrige) Strompfaddichte auf. 


\subsection{LSMO - Einkristall bei $122 \mathrm{~K}$}

Entsprechend der in Kapitel 5.2 vorgestellten Messungen an einer dünnen LCMO - Schicht sollen im Folgenden die elektronischen Eigenschaften eines $\mathrm{La}_{0.8} \mathrm{Sr}_{0.2} \mathrm{MnO}_{3}-$ Einkristalls ${ }^{19}$ untersucht werden. Das geschichtete Wachstum von dünnen Filmen auf verschiedenen Substraten kann zu Verspannungen und somit zu veränderten elektronischen Eigenschaften des Films führen. Bei einem Einkristall handelt es sich um eine sauber strukturierte Bulkprobe die eine starke chemische Homogenität aufweist.

a)

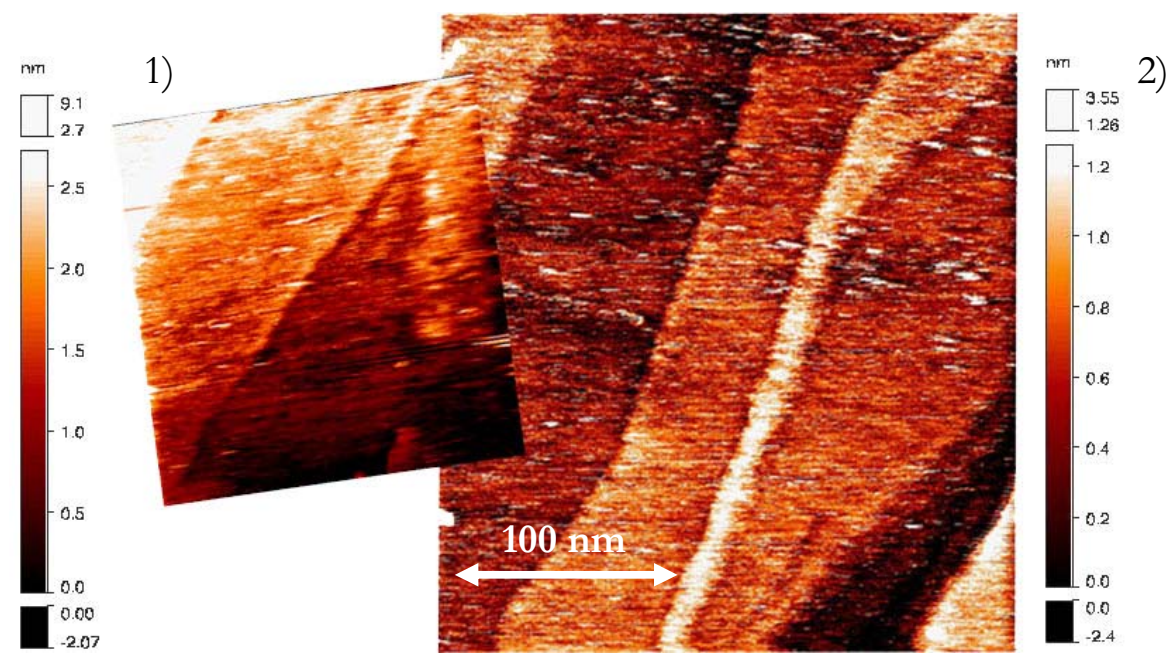

b)

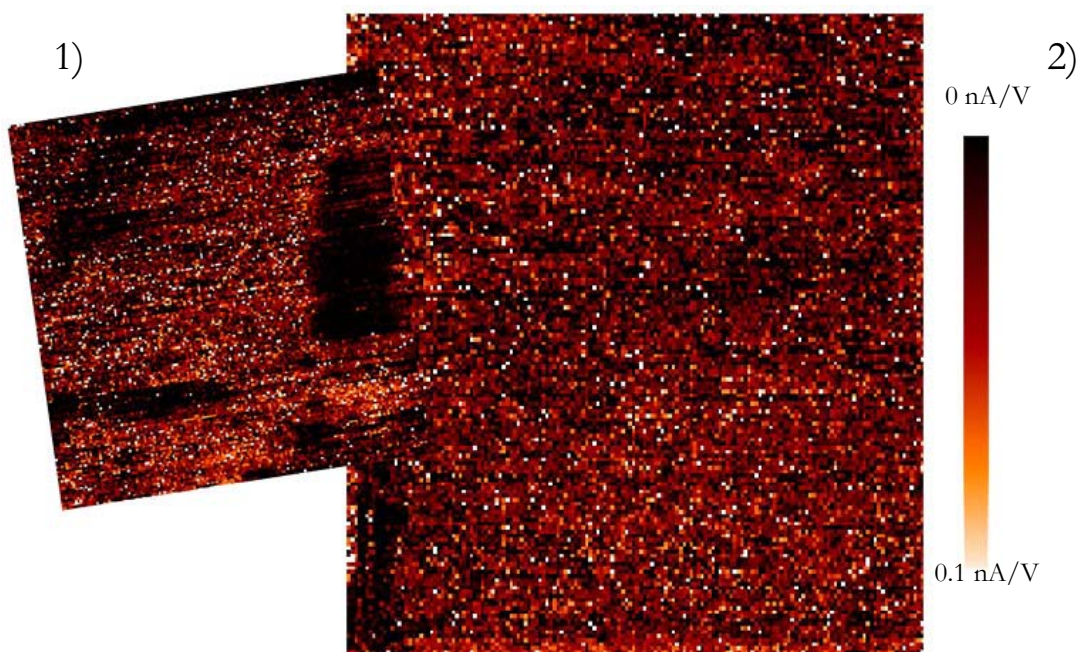

Abbildung 5.6: In (a) sind in 1) und 2) STM - Aufnahmen bei $122 \mathrm{~K}$ von der Oberfläche des LSMO Einkristalls abgebildet $\left(U_{T}=-1 \mathrm{~V} ; I_{T}=0.3 \mathrm{nA}\right)$. Atomare Stufen sind deutlich $\mathrm{zu}$ erkennen. Aufgrund thermischer Drift besteht eine Winkeländerung zwischen den nacheinander aufgezeichneten Bildern 2) und 1). Die zu (a) gehörenden Leitwertmessungen sind in (b) dargestellt. Deutlich sind unterschiedlich leitfähige Bereiche zu erkennen.

\footnotetext{
${ }^{19}$ Die Probe wurde von I. Troyanchuk (Academy of Science - Weißrussland) zur Verfügung gestellt
} 
Das Fehlen von 1 -und 2 -dimensionalen Gitterdefekten (z.B. Korngrenzen) und von Spannungen soll an dieser Stelle ausgenutzt werden, um die Domänenstruktur zu analysieren. Dementsprechend wurde der LSMO - Einkristall auf Phasenseparation hin untersucht.

Messungen mit einem SQUID - Magnetometer ergaben ein $T_{C}=308 \mathrm{~K}^{20}$. Als Messtemperatur wurde $T=122 \mathrm{~K}$ gewählt.

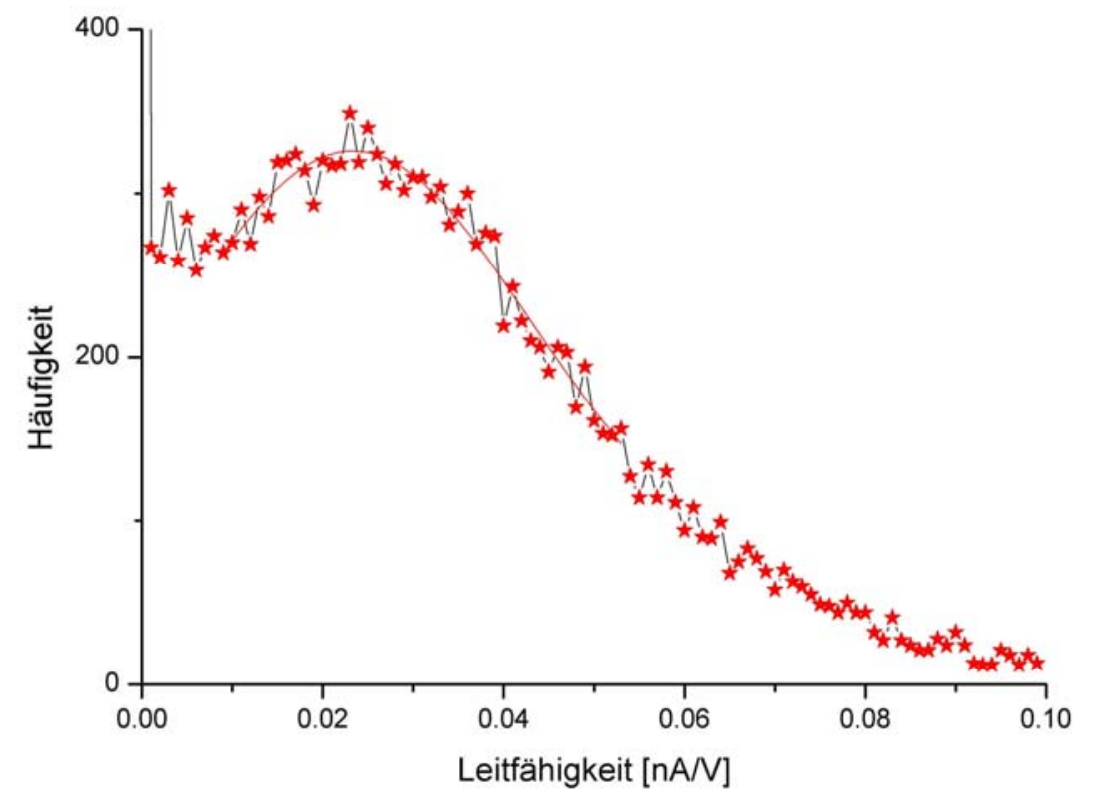

Abbildung 5.7: Häufigkeitsverteilung der bestimmten Leitfähigkeiten entsprechend der Messung dargestellt in Abbildung 5.6 b1). Das Maximum bei $2.3 \cdot 10^{-2} \mathrm{nA} / \mathrm{V}$ wurde mit Hilfe einer Gaußschen Verteilung bestimmt (rote durchgezogene Linie).

Die Ergebnisse der Untersuchungen, dargestellt in Abbildung 5.6 (a), zeigen zwei nacheinander gemessene Topographien, wobei aufgrund von thermischer Drift eine leichte Verdrehung der beiden Bilder zueinander entstanden ist. Beide Aufnahmen zeigen atomare Stufen des Einkristalls. In (b) sind die zu (a) gehörenden Leitwertkarten dargestellt. Dunkle (helle) Farben signalisieren eine niedrige (hohe) Leitfähigkeit entsprechend der angefügten Skala. Zu erkennen ist, dass sich die einzelnen Terrassen untereinander elektronisch nicht unterscheiden. Der Großteil der Oberfläche zeigt eine homogene Leitfähigkeit. Es existieren aber Unterschiede in beiden Auftragungen. Deutlich sind Domänen zu erkennen, die eine schlechtere Leitfähigkeit aufweisen und sich von den leitfähigen Bereichen abgrenzen.

Eine Analyse der Leitfähigkeitsverteilung in Form eines Histogramms (Abbildung 5.7) für die Werte aus b1) zeigt ein ausgeprägtes Maximum der Leitfähigkeit bei $2,3 \cdot 10^{-2} n \mathrm{~A} / \mathrm{V}$. Es ist vom Kurvenverlauf mit der in Abbildung 5.3 dargestellten Häufigkeitsverteilung (LCMO auf MgO) vergleichbar. Für den Übergang vom Maximum in Richtung Isolation nehmen die Häufigkeiten

${ }^{20}$ Die Messung wurde von B. Sass, I. Physikalisches Institut, Universität Göttingen durchgeführt. 
erwartungsgemäß ab. Ab ca. $6 \cdot 10^{-3} n A / V$ beginnen die Werte des Histogramms in Richtung Isolation wieder deutlich anzusteigen, bis sie bei $0 \mathrm{nA} / \mathrm{V}$ in einem Peak enden (außerhalb der Darstellung). Dieses Verhalten kann mit der Koexistenz zweier Verteilungen innerhalb eines Histogramms erklärt werden und ist ein Anzeichen für vorliegende Phasenseparation.

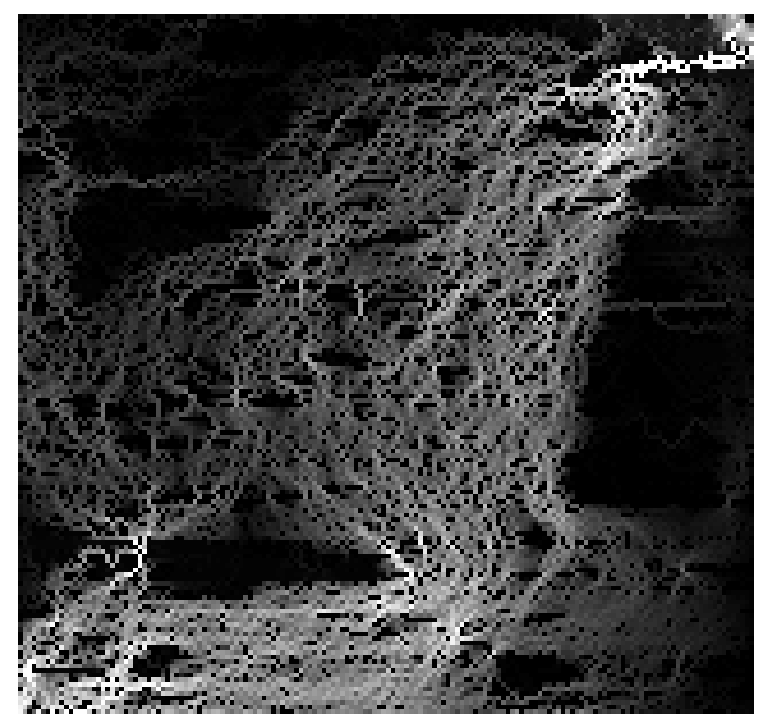

Abbildung 5.8: Stromdichtenverteilung simuliert an der Messung aus Abbildung 5.6 (b1)

Die Ergebnisse der Leitwertkarte aus Abbildung 5.6 b1) wurden zur Berechnung der Stromdichte und deren Darstellung genutzt. Der Verlauf der Pfade ist verglichen mit den Ergebnissen an LCMO aus Kapitel 5.2 ebenfalls als homogen zu bezeichnen und wird nur stellenweise durch isolierende Domänen gestört. Auch dieses Ergebnis zeigt auf, dass eine Phasenseparation am Einkristall vorliegt. 


\subsection{LSMO auf MgO}

In den beiden vorherigen Kapiteln 5.2 und 5.3 wurden die Ergebnisse an einer LCMO - Schicht und an einem LSMO - Einkristall vorgestellt, wobei die Temperatur konstant gehalten wurde. Im Folgenden werden die lokalen elektronischen Eigenschaften eines $\mathrm{La}_{0.7} \mathrm{Sr}_{0.3} \mathrm{MnO}_{3}-\mathrm{Films}^{21}$ von $48 \mathrm{~K}$ bis Raumtemperatur untersucht. Die LSMO - Schicht mit einer Dicke von $50 \mathrm{~nm}$ wurde durch reaktive Sputterdeposition auf einem $\mathrm{MgO}$ - Substrat präpariert [73; 74].

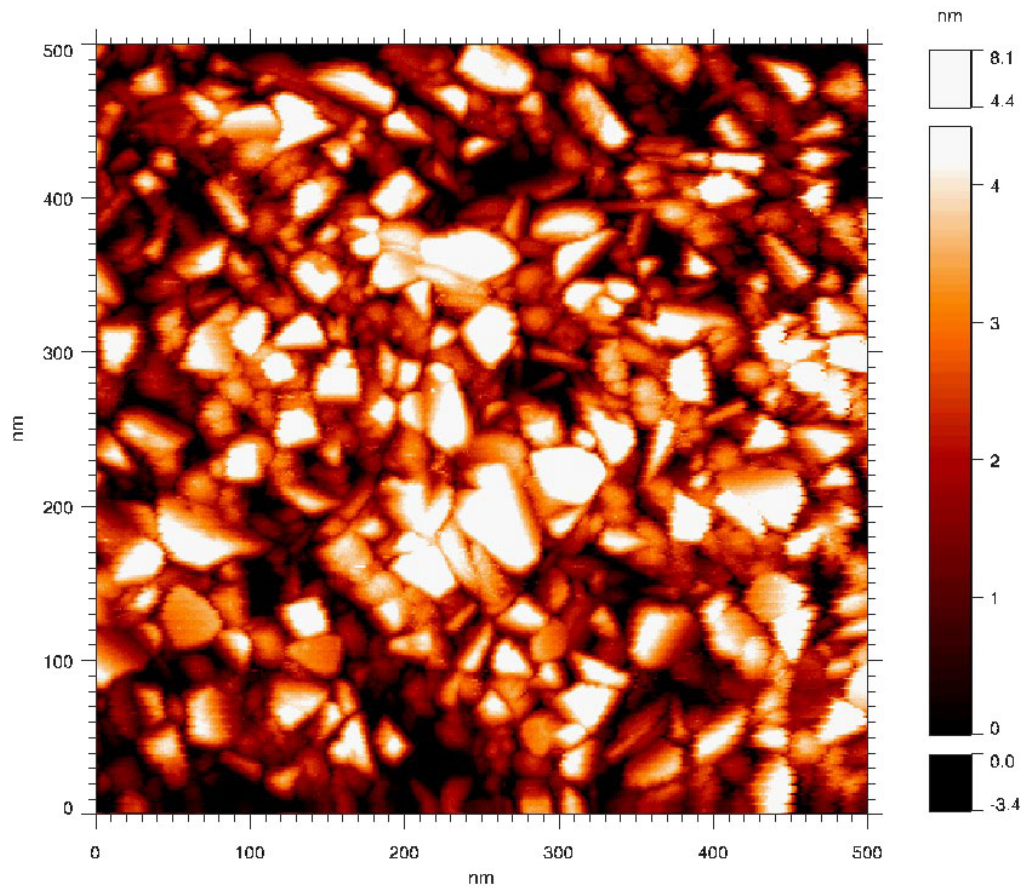

Abbildung 5.9: STM - Bild eines gesputterten LSMO - Films bei $150 \mathrm{~K}$. Die Oberfläche wurde mit $U_{T}=1 \mathrm{~V}, I_{T}=0.3 \mathrm{nA}$ und einem Scanbereich von $500 \times 500 \mathrm{~nm}^{2}$ abgerastert. Die RMS Rauhigkeit berechnet sich zu $1,5 \mathrm{~nm}$.

Abbildung 5.9 zeigt ein bei $T=150 \mathrm{~K}$ aufgenommenes STM - Bild mit einer Ausschnittsgröße von $(500 \mathrm{~nm})^{2}$. Es zeigt eine Mikrostruktur mit einer durchschnittlichen Korngröße von $35 \mathrm{~nm}$. Die RMS - Rauhigkeit ergibt sich zu 1,5 nm. Die Körner sind ungleichmäßig geformt und besitzen eine breite Größenverteilung. Deutlich sind Unterschiede zu der in Abbildung 5.2 (a) dargestellten Schichtoberfläche zu erkennen. Diese Differenzen sind auf die unterschiedlichen Präparationstechniken (MAD und Sputtern) zurückzuführen und werden im Weiteren nicht diskutiert.

\footnotetext{
${ }^{21}$ Die Probe und die durchgeführte Transportmessung stammt von Y. Luo, I. Physikalisches Institut, Universität Göttingen
} 


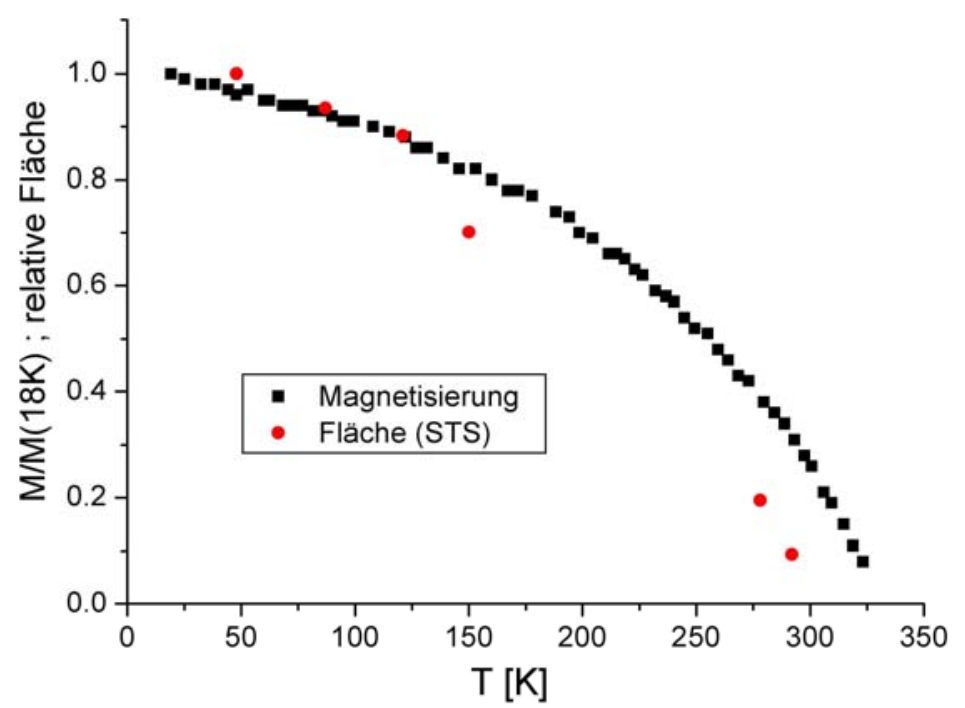

Abbildung 5.10: Magnetisierungsmessung (schwarze Rechtecke) im Temperaturbereich von $20 K$ bis $325 K$. Zusätzlich ist der prozentuale Anteil der als metallisch leitfähig definierten Domänen aus den STS - Messungen dargestellt (rote Kreise). Es existiert eine sehr gute Übereinstimmung der beiden Kurvenverläufe bei Definition nur eines Schwellwertes $\left(S=5,9 \cdot 10^{-3} n A / V\right.$ ). Beide Kurven sind auf ihren Maximalwert normiert worden und stellen somit relative Werte dar.

Parallel zu dieser und weiteren topographischen Aufnahmen, die einem Temperaturbereich von $48 \mathrm{~K}$ bis $292 \mathrm{~K}$ entstammen, sind gerastert Kennlinien aufgezeichnet worden. Um dort metallisch leitfähige Bereiche von isolierenden trennen zu können, wird der Schwellwert $S$ eingeführt. Er definiert sich aus der Anpassung der leitfähigen Bereiche aus den STS-Messungen (2 dimensional) an die an dieser Schicht gemessenen Magnetisierungskurve (3 dimensional) und bestimmt sich zu $S=5,9 \cdot 10^{-3} \mathrm{nA} / \mathrm{V}$. Hierbei wird die Magnetisierung bei tiefen Temperaturen als proportional zu der Gesamtfläche der jeweils leitfähigen Domänen angenommen. Der prozentuale Flächenanteil der leitfähigen Bereiche soll zumindest bei tiefen Temperaturen deckungsgleich mit der Magnetisierungsmessung sein. Wie aus Abbildung 5.10 ersichtlich, liegt eine sehr gute Übereinstimmung bei Definition nur eines Schwellwertes zwischen den Flächenverhältnissen (rot) und der Magnetisierung (schwarz) vor. Abweichungen nahe $T_{C}$ durch mögliche Temperatureinflüsse auf die Magnetisierung der einzelnen leitfähigen Bereiche werden hierbei vernachlässigt (Näheres siehe Kapitel 6). In Abbildung 5.11 sind die Leitfähigkeitsverteilungen der LSMO - Schicht für $48 \mathrm{~K}, 87 \mathrm{~K}, 121 \mathrm{~K}, 150 \mathrm{~K}, 278 \mathrm{~K}$ und $292 \mathrm{~K}$ dargestellt. Um diese Form der Leitwertkarten zu generieren, werden die gerasterten Ausgangsleitwerte über eine Gaußsche Verteilungsfunktion geglättet und dann durch den Schwellwert voneinander getrennt. Isolierende Bereiche sind schwarz dargestellt $(d I / d V<S)$, während weiße Flächen ein metallisch leitfähiges Verhalten zeigen $(d I / d V>S)$. 
$48 \mathrm{~K}$

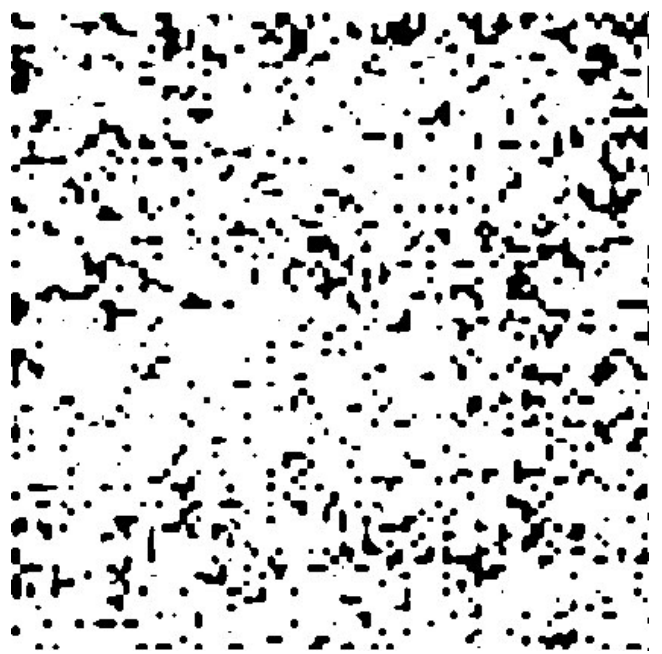

$121 \mathrm{~K}$

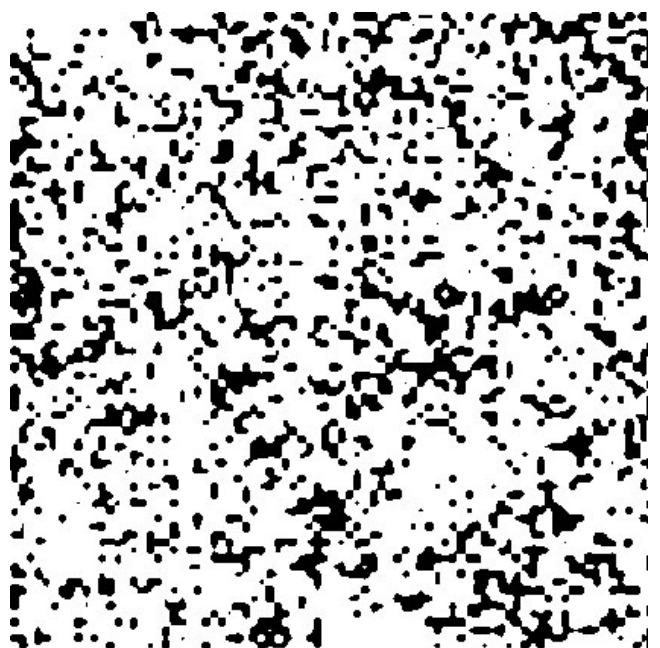

$278 \mathrm{~K}$

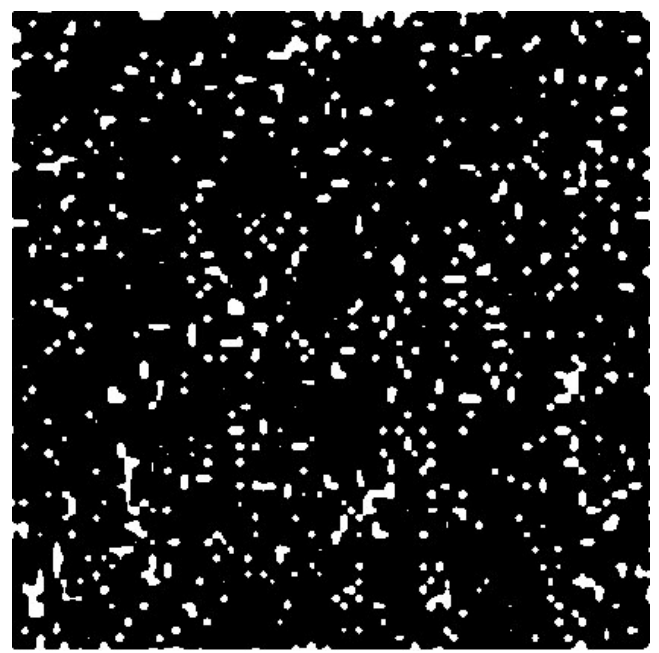

$87 \mathrm{~K}$

F.

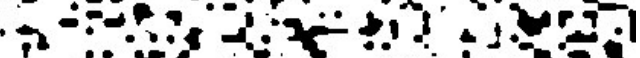

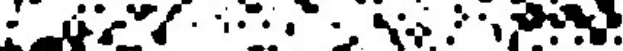

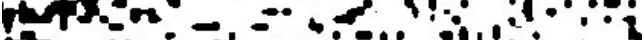

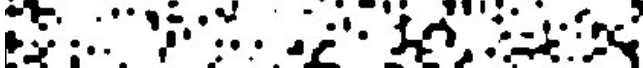
T.

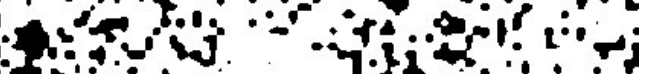
a. 4. 4. F,

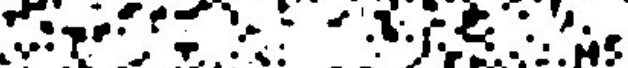

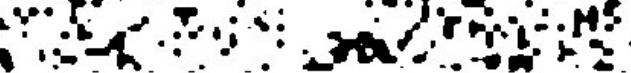

$150 \mathrm{~K}$

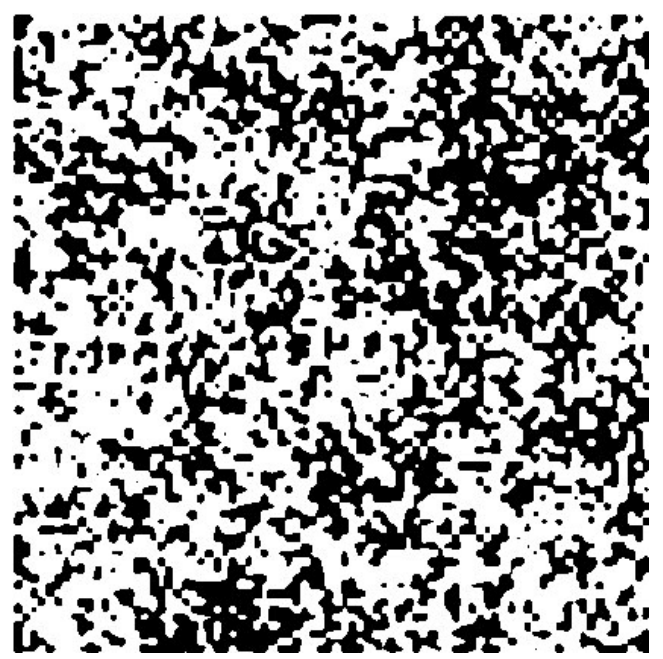

$292 \mathrm{~K}$

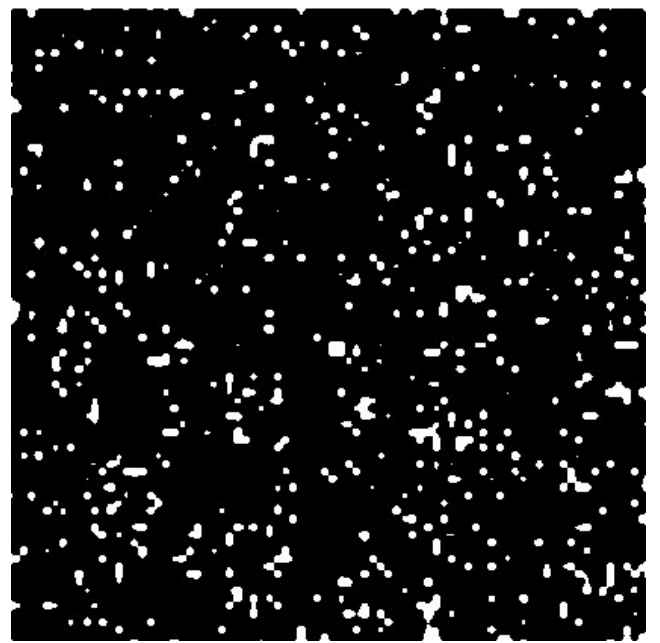

Abbildung 5.11: Leitfähigkeitsverteilungen mit einer Größe von $500 \times 500 \mathrm{~nm}^{2}$, aufgenommen bei verschiedenen Temperaturen. Schwarz: isolierende Bereiche $\left(d I / d V<5,9 \cdot 10^{-3} n A / V\right.$; Weiß: metallische Regionen $\left(d I / d V>5,9 \cdot 10^{-3} n A / V\right)$ 
Eine Betrachtung der Leitfähigkeitsverteilung, dargestellt als Histogramm in Abbildung 5.12, unterstreicht die Wahl des Schwellwertes S. Die Kurvenverläufe bei tiefen Temperaturen entsprechen einer Gaußverteilung und besitzen ein „leitfähiges“ Maximum oberhalb von $1 \cdot 10^{-2} \mathrm{n} / \mathrm{V}$. Betrachtet man hingegen die Messungen bei hohen Temperaturen nahe dem MIT, entspricht der Verlauf eher einem exponentiellen Abfall (erkennbar im linearen Verlauf des Graphen bei logarithmischer Auftragung) mit Isolation $(0 n A / V)$ als einzigem Maximum. Es bilden sich zwei verschiedene Verteilungen heraus, die als isolierend bzw. leitfähig erklärt werden können und das Vorliegen zweier unterschiedlicher Phasen kennzeichnen. Die Abnahme der einen hat die Zunahme der anderen zur Folge und erklärt den Kurvenverlauf für $150 \mathrm{~K}$. Das Maximum ist zu niedrigerer Leitfähigkeit hin verschoben. Der Schnittpunkt der beiden dominierenden Verteilungen (rote Senkrechte) liegt bei $S^{\prime}=4,5 \cdot 10^{-3} \mathrm{nA} / \mathrm{V}$, sehr nahe dem oben bestimmten Schwellwert $S$ (blau eingezeichnet).

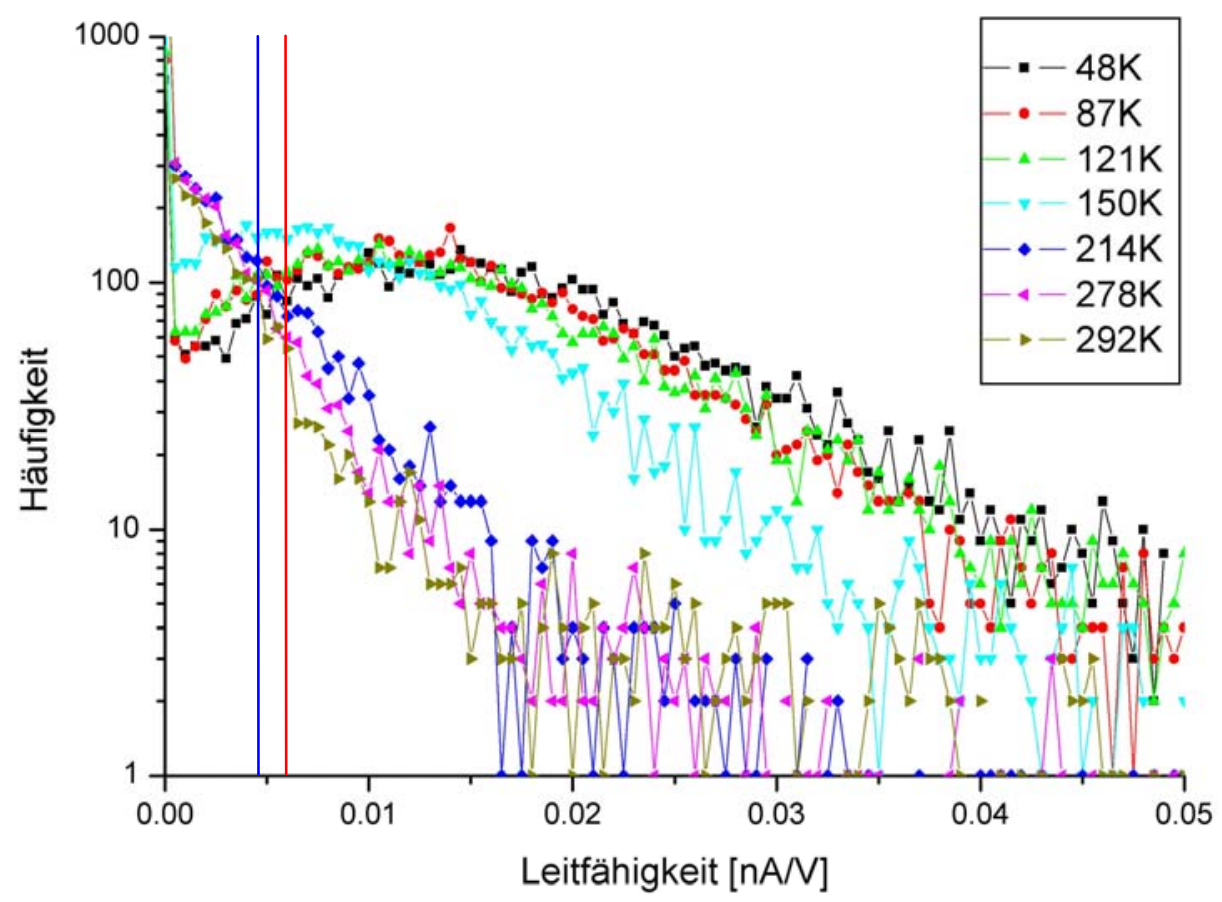

Abbildung 5.12: Leitfähigkeitsverteilungen der unterschiedlichen Messungen von $48 K$ bis $292 K$. Zwei vorherrschende Kurvenverläufe sind zu erkennen. Als rote Linie ist der aus der Magnetisierungsmessung bestimmte Schwellwert $S=5,9 \cdot 10^{-3} n A / V$ eingezeichnet; die blaue Linie stellt den Schnittpunkt der beiden dominierenden Kurvenverläufe $\left(S^{\prime}=4,5 \cdot 10^{-3} n A / V\right)$ dar. 
Die Leitfähigkeitsverteilung bei $48 \mathrm{~K}$ (Abbildung 5.11) zeigt eine zusammenhängende, leitfähige Fläche (weiß) mit wenigen isolierenden Inseln (schwarz). Durch die Erhöhung der Temperatur auf $87 \mathrm{~K}$ nehmen die leitfähigen Domänen zugunsten der isolierenden Phase leicht ab. Die Anfangsstadien eines filamentartigen, isolierenden Netzwerkes sind zu erkennen. Die mittlere Breite eines Filaments liegt mit $21 \mathrm{~nm}$ deutlich unter der oben bestimmten Korngröße. Dieses Ergebnis zeigt, dass die entstehenden Domänenstrukturen nicht direkt mit der vorhandenen Mikrostruktur korrelieren, es kann auf intrinsische Effekte zurückgeführt werden. Erhöht man die Temperatur weiter, nimmt die Vernetzung immer weiter zu. In der Verteilung bei $150 \mathrm{~K}$ existieren noch zusammenhängende, metallische Pfade, die einen Stromfluss und somit niedrigen Widerstand gewährleisten können. Bei Raumtemperatur sind nur noch wenige metallische Inseln vorhanden. Der Großteil der Probe besitzt nun ein schlechtleitendes bzw. isolierendes Verhalten. Der mittlere Tunnelwiderstand der metallischen Bereiche oberhalb des Schwellwertes ist in Abbildung 5.13 als Funktion der Temperatur aufgetragen. Er nimmt mit wachsender Temperatur nur schwach zu - ein Ergebnis, wie es aus den Rechnungen von Stratton [67] für freie Elektronen bzw. für reine Metalle bekannt ist. Dieses Verhalten kann die Form des Metall - Isolator Übergangs, entsprechend der Transportmessung aus Abbildung 5.13 (rote Linie), nicht erklären. Aus den beiden Darstellungen für $150 \mathrm{~K}$ und $278 \mathrm{~K}$ (Abbildung 5.11) ist ersichtlich, dass die Strompfade, die bei $150 \mathrm{~K}$ noch existieren, bei $278 \mathrm{~K}$ nicht mehr vorhanden sind. Es liegt keine Perkolation mehr von metallischen Bereichen vor. Der Strom muss nun stellenweise durch schlechtleitende Bereiche fließen, um von einer metallischen Domäne zur nächsten zu gelangen. Damit ein Vergleich ${ }^{22}$ zwischen den Tunnelexperimenten und der Transportmessung durchgeführt werden kann, interpretieren wir die gemessenen Tunnelwiderstände als ein zusammenhängendes, 2 dimensionales Netzwerk (entsprechend Kapitel 4.2) und berechnen dessen Gesamtwiderstand. Die Ergebnisse der Simulationen sind in Abbildung 5.11 als blaue Sterne gekennzeichnet. Ihre Entwicklung entspricht sehr gut dem Kurvenverlauf der Transportmessung und spiegelt den Einfluss von perkolativen Strompfadbildungen nahe der Übergangstemperatur wider. Die drei dargestellten Stromverlaufsbilder aus Abbildung 5.13 verdeutlichen $\operatorname{dies}^{23}$. Bei den Temperaturen von $48 \mathrm{~K}$ und $150 \mathrm{~K}$ liegt eine relativ homogene Stromverteilung vor. Der Stromverlauf breitet sich, entsprechend der homogenen Verteilung von metallischen Bereichen, gleichmäßig über den gemessenen Bereich aus. Bei $278 \mathrm{~K}$ muss der Strom auch isolierende Bereiche durchdringen. Er sucht sich einen Weg mit dem niedrigsten

\footnotetext{
22 An dieser Stelle soll angemerkt werden, dass ein direkter Vergleich der Werte von Transportwiderstands- und Tunnelwiderstandsmessungen nur bedingt interpretiert werden kann, da sich beide Messmethoden in den vorherrschenden physikalischen Prozessen voneinander unterscheiden.

${ }^{23}$ Die Farbskalen der drei Bilder orientieren sich jeweils am maximalen Stromfluss - ein direkter Vergleich des maximalen Stromflusses ist hier also nicht möglich.
} 
Widerstand und bildet einen einzigen „Stromfaden“. Der Einfluss der isolierenden Phase hat eine Zunahme des Gesamtwiderstandes zur Folge.

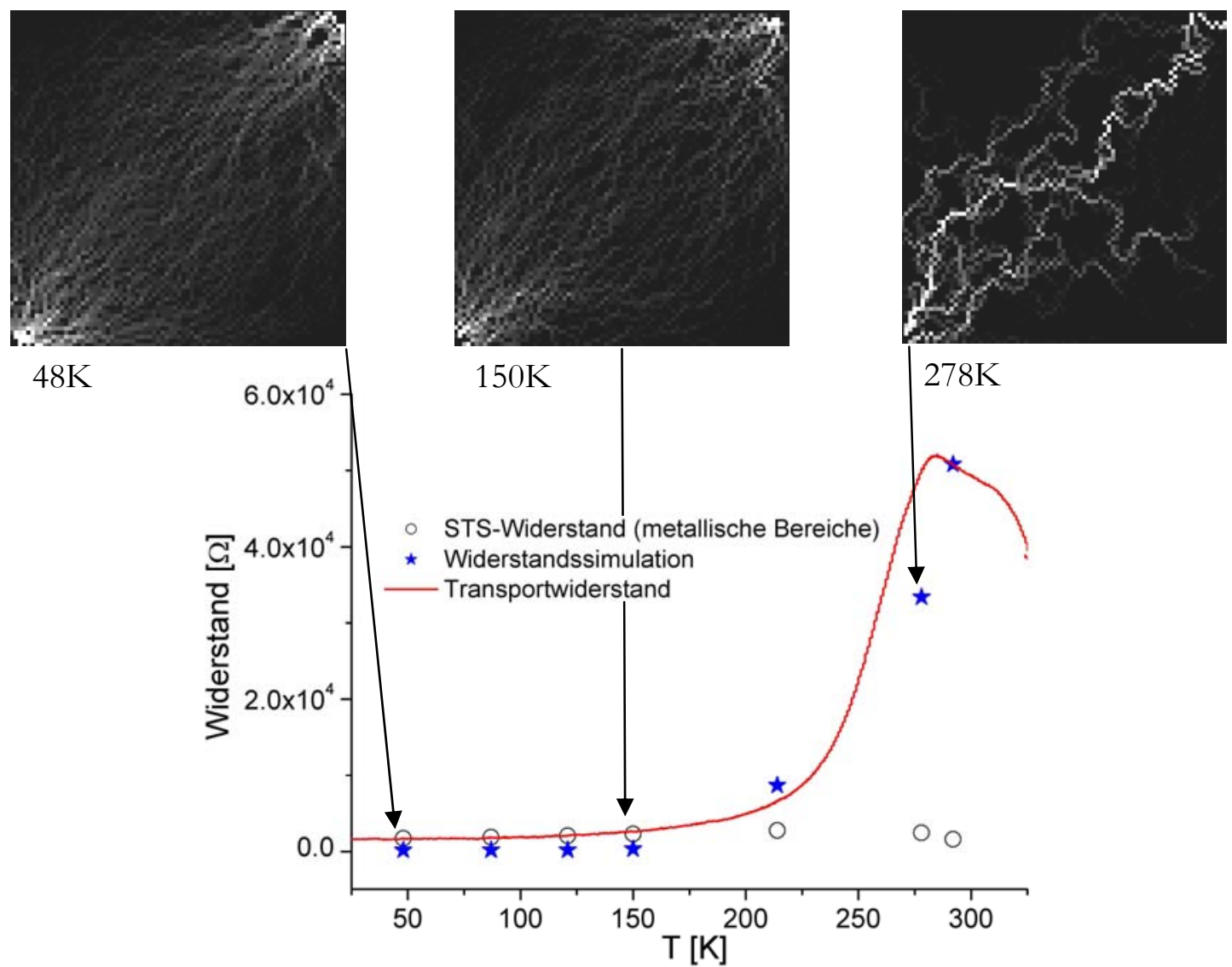

Abbildung 5.13: Transportwiderstand (rote Linie); normierte Tunnelwiderstände der leitfähigen Domänen (Kreise) und normierte Netzwerkwiderstände der Simulation (blaue Sterne) mit drei exemplarisch dargestellten Strompfadauftragungen bei $48 K, 150 K$ und $278 K$ [75].

Ohne die Existenz intrinsischer Inhomogenitäten wäre ein direkter Sprung im Widerstandsverlauf vom metallischen zum isolierenden Verhalten bei $T_{C}$ zu erwarten. Die vorliegenden Ergebnisse zeigen jedoch deutlich den Zerfall in leitfähige und isolierende Domänen und besitzen somit Erklärungspotential hinsichtlich der Form des Kurvenverlaufes der durchgeführten Transportmessungen. Aufgrund der vorliegenden Phasenseparation liegt kein scharfer Übergang bei $T_{C}$ vor. Weiterführende Messungen an dieser Probe, um auch den Bereich oberhalb des Metall - Isolator - Übergangs simuliert darzustellen, liegen technisch bedingt nicht vor. Eine entstehende Sauerstoffdegradation würde den Oberflächenwiderstand ändern und die Ergebnisse verfälschen. 


\subsection{LSMO auf Iridium}

Manganatfilme auf isolierenden Substraten können STM - und STS - Messungen durch den hohen intrinsischen Schichtwiderstand erschweren, da die Proben nur am Rand geerdet werden. Um bei den teils hochohmigen LSMO und LCMO - Schichten den Massekontakt über den Materialfilm hin zum Tunnelkontakt zu verbessern, ist ein metallisch leitfähiges Substrat, das ein epitaktisches Schichtwachstum vergleichbar mit dem auf $\mathrm{MgO}$ vorweist, besser geeignet. Der Massekontakt besteht nicht mehr aus dem lateralen Manganatfilm, sondern kann direkt über das Substrat bewerkstelligt werden. Iridium bietet sich für Manganatschichten als Kontaktmaterial $\mathrm{an}^{24}$ und wird im Folgenden als Metallschicht zwischen dem Manganat und dem Substrat eingesetzt.

Die Oberfläche eines $35 \mathrm{~nm}$ dicken LSMO - Films auf $20 \mathrm{~nm}$ Iridium ist Abbildung 5.14 dargestellt. Der Messbereich von $500 \times 500 \mathrm{~nm}^{2}$ ist bei einer Temperatur von $37 \mathrm{~K}$ aufgenommen worden. Die mittlere Korngröße $(21 \mathrm{~nm})$ ist im Vergleich zu der in Kapitel 5.4 vorgestellten Messung etwas geringer. Ein Ergebnis, das auf die niedrigere Schichtdicke zurückzuführen ist. Die RMS - Rauhigkeit mit 1,7 nm ist vergleichbar.

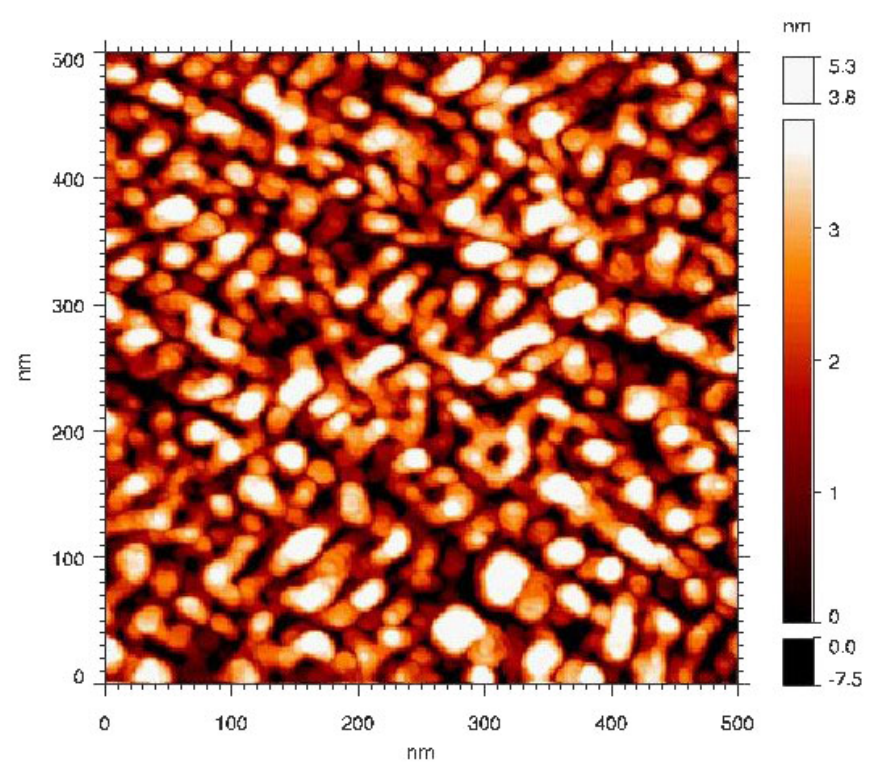

Abbildung 5.14: Topographisches STM - Bild eines $35 \mathrm{~nm}$ dicken LSMO - Films präpariert auf $20 \mathrm{~nm}$ Iridium (beides gesputtert). Als Substrat dient MgO. Der Ausschnitt von $500 \times 500 \mathrm{~nm}^{2}$ ist bei einer Temperatur von $37 K$ aufgenommen worden.

\footnotetext{
24 Präparationsmethoden und weiterführende Informationen sind in [76] zu finden. Die Probe, die Transportmessung und die Magnetisierungsmessung stammen von A. Giske, I. Physikalisches Institut, Universität Göttingen
} 
Um einen Einfluss der Iridium - Kontaktschicht auf die elektronischen Eigenschaften des LSMO

- Films auszuschließen, werden gerasterte Kennlinienanalysen und die Bestimmung des Schwellwertes entsprechend der im letzten Kapitel vorgestellten Messungen durchgeführt und mit diesen Ergebnissen verglichen.
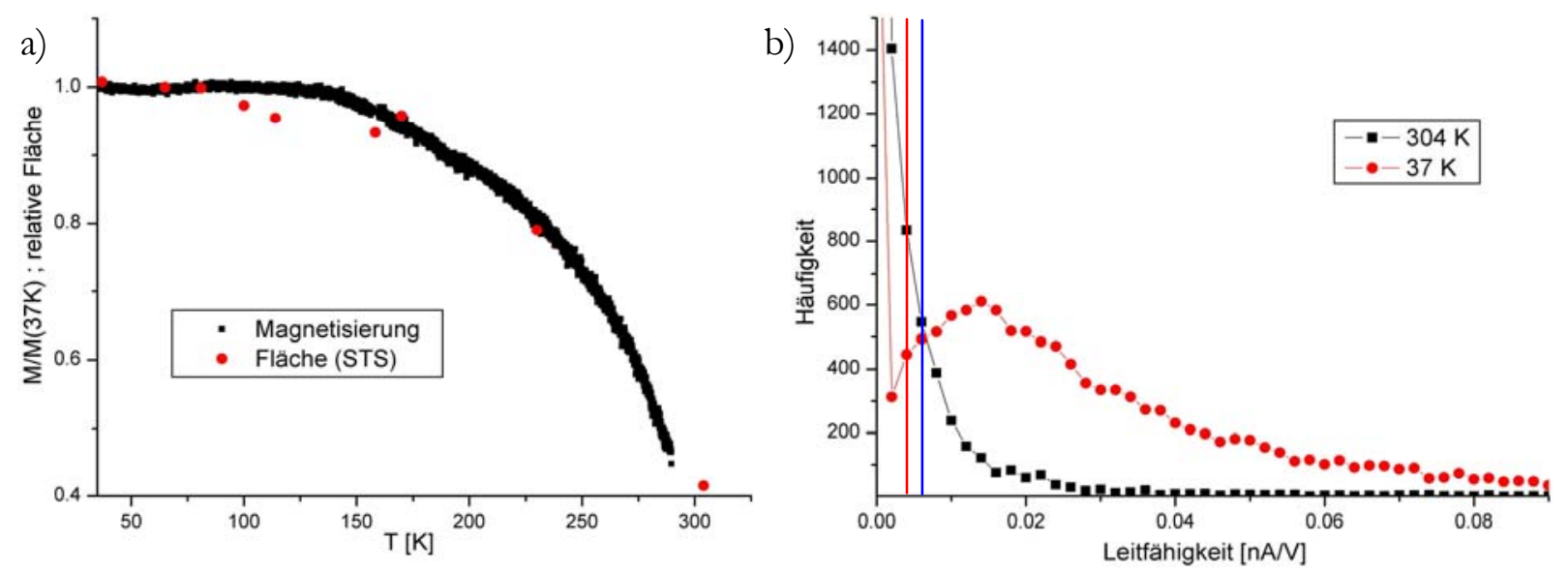

\begin{abstract}
Abbildung 5.15: a) Magnetisierungsmessung und Anpassung der metallisch leitfähigen Flächen entsprechend Abbildung 5.10. Der Schwellwert wird hier zu $S=4,0 \cdot 10^{-3} \mathrm{nA} / \mathrm{V}$ bestimmt.

b) Histogramm für Messung bei $37 K$ (rot) und 304 K (schwarz). Die leitfähige Phase zeigt bei tiefer Temperatur ein Maximum bei $1,4 \cdot 10^{-2} \mathrm{nA} / \mathrm{V}$. Dieses ist bei $304 \mathrm{~K}$ nicht mehr vorhanden - einzig der scharfe Übergang zu hohen Werten nahe Isolation ist auffallend. Der Schwellwert $S$ aus (a) ist als rote senkrechte Linie dargestellt. Der Schnittpunkt beider Graphen liegt bei $S^{\prime}=6,3 \cdot 10^{-3} \mathrm{nA} / \mathrm{V}$ und ist blau gekennzeichnet.
\end{abstract}

In einem Temperaturbereich von $37 \mathrm{~K}$ bis $304 \mathrm{~K}$ sind für verschiedene Temperaturen Leitwertkarten aufgenommen worden. Die Trennung von isolierenden und leitfähigen Bereichen geschieht wiederum durch die Bestimmung eines Schwellwertes aus dem Fit der metallischen Fläche an die Magnetisierungskurve. In Abbildung 5.15 (a) ist das Ergebnis für diese Anpassung gezeigt. Die roten Punkte stellen die bestimmten Flächenanteile der metallischen Phase für den Schwellwert $S=4,0 \cdot 10^{-3} \mathrm{n} A / V$ bei unterschiedlichen Temperaturen dar und zeigen eine gute Übereinstimmung mit der schwarz gezeichneten Magnetisierungsmessung.

Bei tiefer Temperatur ist davon auszugehen, dass der größte Teil der Probe metallisches Verhalten zeigt. Im Gegensatz dazu ist bei hohen Temperaturen (nahe $T_{\partial}$ ) fast nur noch isolierendes Verhalten nachweisbar. Vergleicht man die Leitfähigkeitsverteilung der gerasterten Kennlinienanalyse, aufgenommen bei tiefer und hoher Temperatur, zeigen sich die aus Abbildung 5.12 bekannten Verläufe der Graphen. Das Ergebnis bei $37 \mathrm{~K}$ (rot dargestellt in 
Abbildung 5.15 (b)) zeigt ein deutliches Maximum bei 1,4 $10^{-2} n A / V$. Der Verlauf der Kurve bei $304 \mathrm{~K}$ zeigt dieses Maximum nicht mehr. Vielmehr liegt ein exponentieller Abfall nahe $0 \mathrm{nA} / \mathrm{V}$ (Isolation) vor. Der Schnittpunkt beider Graphen bestimmt sich zu $S^{\prime}=6,3 \cdot 10^{-3} \mathrm{nA} / \mathrm{V}$ (blau dargestellt) und stimmt recht gut mit dem aus der Magnetisierungsmessung ermittelten Schwellwert überein (siehe Tabelle). Ein Vergleich dieser Werte mit den Ergebnissen des letzten Kapitels zeigt eine sehr gute Konsistenz mit dem Mittelwert $5,2 \cdot 10^{-3} \mathrm{nA} / \mathrm{V}$.

\begin{tabular}{|l|l|c|}
\hline Probe & Bestimmungsmethode & Schwellwert (nA/V) \\
\hline LSMO auf MgO & Fit an Magnetisierungsmessung & $5,9 \cdot 10^{-3}$ \\
\hline LSMO auf MgO & Schnittpunkt der Histogramme & $4,5 \cdot 10^{-3}$ \\
\hline LSMO auf Iridium & Fit an Magnetisierungsmessung & $4,0 \cdot 10^{-3}$ \\
\hline LSMO auf Iridium & Schnittpunkt der Histogramme & $6,3 \cdot 10^{-3}$ \\
\hline & Mittelwert: & $\mathbf{5 , 1 7 5} \cdot \mathbf{1 0}^{-3}$ \\
\hline
\end{tabular}

Die Leitfähigkeitsverteilung mit dem angewendeten Schwellwert $S$ sind in Abbildung 5.16 (linke Spalte) für drei verschiedene Temperaturen dargestellt. Weiße Bereiche signalisieren vorliegende Leitfähigkeit $(d I / d V>S)$, schwarze indes Isolation $(d I / d V<S)$. Die Darstellungen besitzen die gleiche Ausschnittsgröße des topographischen STM - Bildes (so.) von $500 \times 500 \mathrm{~nm}^{2}$. Bei $37 \mathrm{~K}$ zeigt die Karte größtenteils leitfähiges Verhalten. Kleine isolierende Domänen sind vorhanden, die anfänglich getrennt voreinander bestehen. Mit steigender Temperatur wachsen diese Bereiche auf Kosten der leitfähigen Phase $(170 \mathrm{~K})$ und koaleszieren schließlich zu einem Netz zusammen (304 K). Es bestehen immer noch leitfähige Domänen, die jedoch nicht mehr zusammenhängend vorliegen. Anhand der Leitfähigkeitskarten wurden Strompfadsimulationen durchgeführt und exemplarisch für die Temperaturen von $37 \mathrm{~K}, 170 \mathrm{~K}$ und $304 \mathrm{~K}$ in Abbildung 5.16 dargestellt. Die Strompfadkarten bei diesen Temperaturen zeigen eine homogene Verteilung des Stromflusses. Einzig die Bereiche, bei denen isolierendes Verhalten vorliegt, zeigen keinen Stromfluss (bei $170 \mathrm{~K}$ mit roten Pfeilen angedeutet). In der Simulation bei $304 \mathrm{~K}$ zeichnen sich einige Strompfade im linken unteren und rechten oberen Bildausschnitt aus. Zum Mittelpunkt des Bildes hin fächern diese Verläufe auf. Sie werden deutlich schwächer, bis sie im Bildmittelpunkt nicht mehr zu erkennen sind. An den simulierten Kontaktpunkten in den Ecken der Messung hat der Stromfluss nur wenige Möglichkeiten um die Probe zu durchdringen. Er muss einige schlecht leitfähige Bereiche durchqueren um auf leitfähige Domänen zu treffen. Dort kann er sich verteilen und weitere Übergangsmöglichkeiten suchen, erkennbar ist dies an der Abnahme der Helligkeitswerte. 

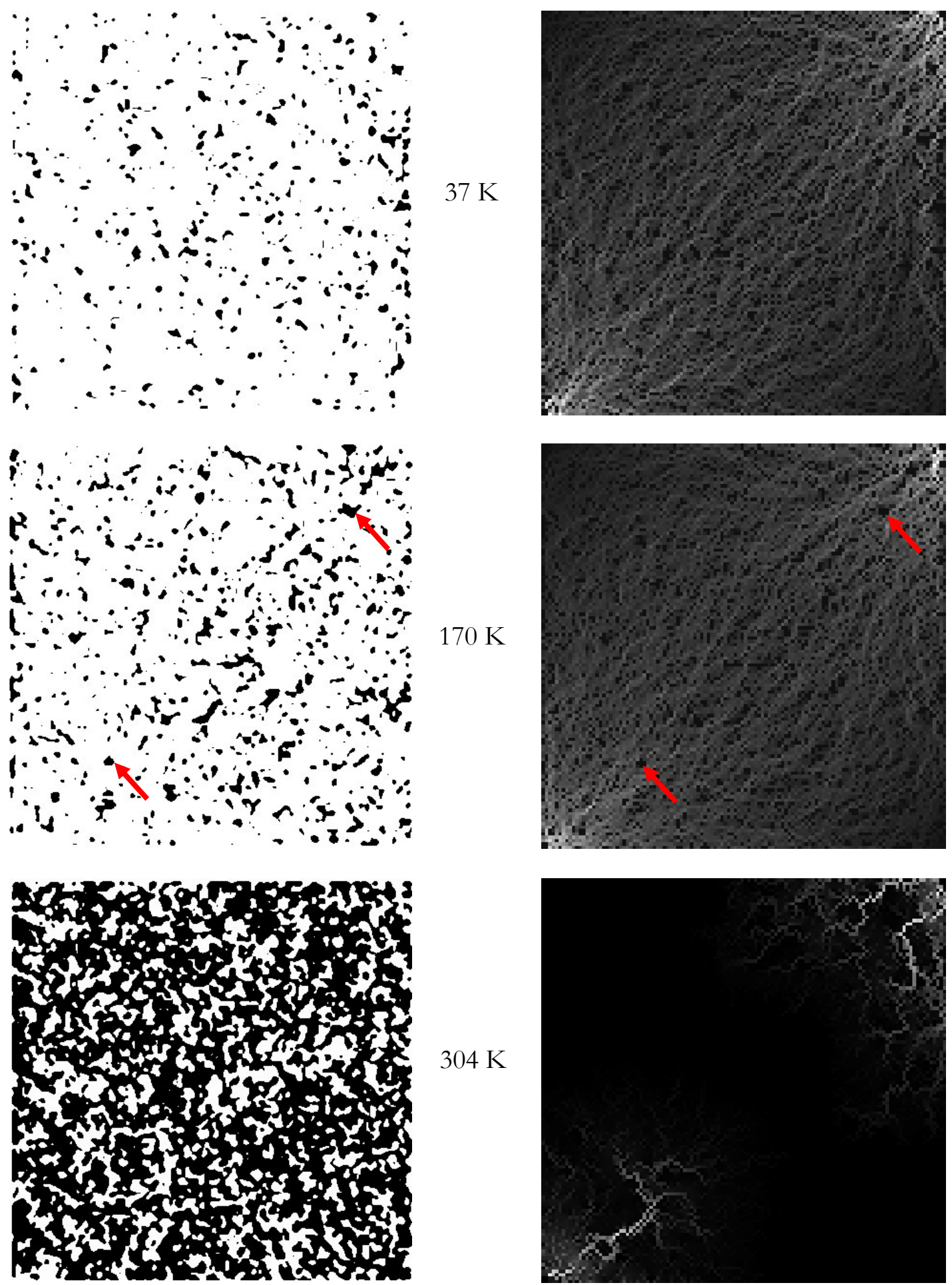

Abbildung 5.16: Linke Spalte: Leitfähigkeitsverteilung mit angewendetem Schwellwert $S=4,0 \cdot 10^{-3} n A / V$ und einer Größe von $500 \times 500 \mathrm{~nm}^{2}$ für verschiedene Temperaturen. Schwarz entspricht isolierenden Bereichen, leitfähige Domänen sind weiß dargestellt.

Rechte Spalte: Stromverlaufskarte bei entsprechender Temperatur. Die roten Pfeile deuten auf isolierende Bereiche hin. 
Die Strompfadbildung über rein metallisch leitfähige Bereiche ist somit zusammengebrochen und es liegt keine Perkolation mehr vor. Die Ergebnisse der Simulationen (blaue Sterne) sind in Abbildung $5.17 \mathrm{im}$ Vergleich mit dem Transportwiderstand (rote Linie) dargestellt. Wenn man die Widerstandsmessung an dem Schichtpaket als vorliegende Parallelschaltung zweier Widerstände betrachtet (Iridium und Manganat) wird ersichtlich, dass der Widerstandsverlauf nicht den für Manganate gewohnten Kurvenverlauf besitzt. Im Tieftemperaturbereich (bis ca. $250 \mathrm{~K}$ ) dominiert die Leitfähigkeit des reinen Metalls (Iridium) und das LSMO liefert nur einen geringen Beitrag. In der Nähe des Metall-Isolator-Übergangs (bei ca. $275 \mathrm{~K}$ ) nimmt der Widerstand des Manganatfilms stark zu und leistet einen geringeren Anteil zur Gesamtleitfähigkeit. Eine geringe Widerstandserhöhung ist das Resultat. Oberhalb des Übergangs nimmt der Widerstand der Manganatschicht wieder ab und senkt den Gesamtwiderstand um einen geringen Betrag ab. Die Erhöhung des Widerstandes durch den Beitrag der Manganatschicht ist qualitativ als ein kleiner „Buckel“ deutlich zu erkennen (roter Pfeil). Bei der Erstellung der Leitwertkarten, bzw. bei der Aufnahme der Kennlinien an der Manganatoberfläche wird nur die Zustandsdichte der LSMO - Schicht an der Oberfläche betrachtet. Das Iridium unterhalb des Manganatfilms ist für die STS - Messung irrelevant. Somit zeigen die Ergebnisse der Simulation den gewohnten Widerstandsverlauf für Manganate: Im Temperaturbereich von $37 \mathrm{~K}$ bis $170 \mathrm{~K}$ ist eine geringe Steigung zu erkennen. Erst bei $230 \mathrm{~K}$ und $304 \mathrm{~K}$ zeigen die Simulationen einen hohen Anstieg im Widerstand.

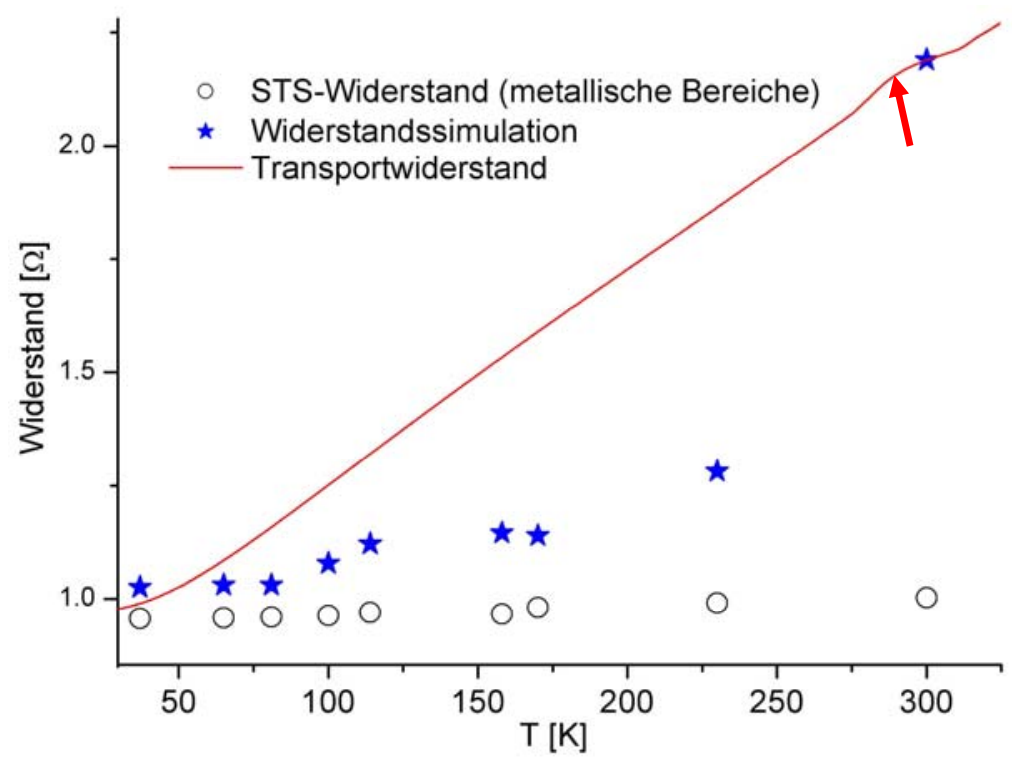

Abbildung 5.17: Transportwiderstand des Schichtpaketes LSMO und Iridium (rote Linie); normierte Tunnelwiderstände der leitfähigen Bereiche (Kreise) und normierte Ergebnisse aus der Simulation (blaue Sterne) 
Die mittleren Tunnelwiderstände der metallischen Bereiche oberhalb des Schwellwertes sind in dem Graphen als weiße Kreise eingezeichnet und zeigen eine nur recht schwache Zunahme des Widerstandes. Ein Ergebnis, das mit LSMO auf MgO aus Abbildung 5.13 vergleichbar ist und dies unterstreicht.

Zusammenfassend zeigen diese Ergebnisse, dass die Iridium-Zwischenschicht die STS Messungen an dem Manganatfilm nicht negativ beeinflusst hat - die Ergebnisse stimmen recht gut mit denen aus dem letzten Kapitel überein. Zusätzlich hat sich die STM - Messfähigkeit am Manganatfilm verbessert.

\subsection{Sauerstoffreduziertes LCMO auf Iridium ${ }^{25}$}

Die beiden Messungen und Analysen an Manganaten aus den vorhergehenden Kapiteln konnten nur bis zum Metall - Isolator - Übergang dargestellt werden. Es ist aber von zusätzlichem Interesse, in welcher Form der Abfall des Widerstandes oberhalb des MIT erklärt werden kann. Da die untersuchten Proben nur für kurze Zeit oberhalb von Raumtemperatur im UHV stabil sind, bzw. aufgrund von Sauerstoffverlust ihre Leitfähigkeit verlieren und nicht reproduzierbare Ergebnisse liefern, kann dieser Temperaturbereich für Messungen im UHV nicht genutzt werden.

a)

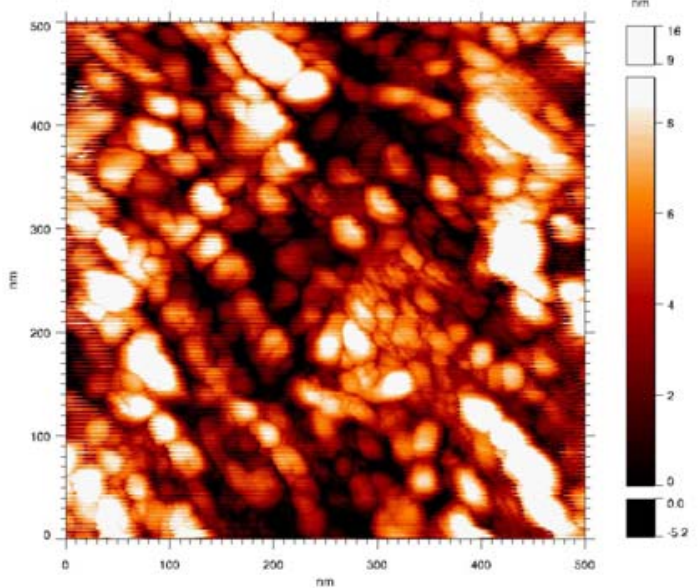

b)

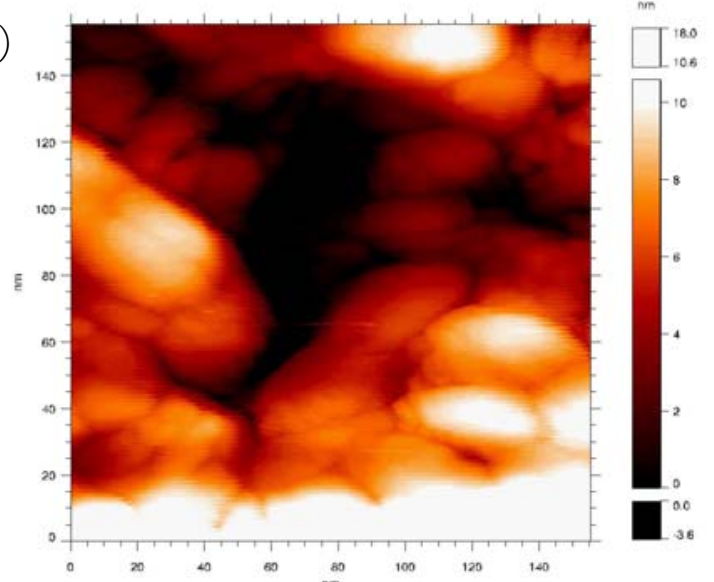

Abbildung 5.18: In (a) ist das STM - Bild eines $45 \mathrm{~nm}$ dicken LCMO - Films auf $25 \mathrm{~nm}$ Iridium bei $51 K$ dargestellt (beide Schichten gesputtert). Die Oberfläche wurde mit $U_{T}=0,7 \mathrm{~V}, I_{T}=0,4 \mathrm{nA}$ und einem Scanbereich von $500 \times 500 \mathrm{~nm}^{2}$ abgerastert. Die RMS - Rauhigkeit berechnet sich zu 2,8 nm. Alle im Folgenden diskutierten und dargestellten Leitwertkarten basieren auf einem Scanbereich von $155 \times 155 \mathrm{~nm}^{2}$. In (b) ist beispielhaft eine Aufnahme bei $239 \mathrm{~K}$ aufgetragen.

\footnotetext{
${ }^{25}$ Die Probe und die durchgeführte Transportmessung stammen von Y. Luo, I. Physikalisches Institut, Universität Göttingen
} 
Dementsprechend muss eine Probe zur Messung herangezogen werden, bei der der MIT weit unterhalb der Raumtemperatur vorliegt. Der Sauerstoff hat aufgrund seiner elektronischen Wechselwirkung mit dem Mangan direkten Einfluss auf die Leitfähigkeit der Probe. Enthält der Manganatkristall nicht die ausreichende Menge an Sauerstoffionen, verschiebt sich der MIT zu tiefen Temperaturen [77]. Damit die Messfähigkeit des Manganatfilms verbessert wird, ist vor der eigentlichen Schichtpräparation eine $25 \mathrm{~nm}$ dicke Iridiumschicht auf dem $\mathrm{MgO}$ - Substrat aufgetragen worden. Es ist nur eine Hälfte des Substrates mit Iridium bedeckt worden, damit bei Transportmessungen keine störenden Einflüsse des Iridiums auftreten, sondern nur der Widerstand der reinen Manganatschicht gemessen werden kann. Als Schichtdicke des LCMO wurde 45nm gewählt. Um eine Manganatprobe mit reduziertem Sauerstoffgehalt zu erhalten, wird bei der Sputterdeposition ein verringerter Sauerstoffpartialdruck (2,5·10 mbar statt normalerweise $5,0 \cdot 10^{-2}$ mbar [77]) eingestellt. Die so präparierte Probe wird in zwei Teile geteilt, wobei die Hälfte ohne Iridium für Transportmessungen herangezogen wurde und eine Verschiebung des Übergangs von $260 \mathrm{~K}$ (bulk) bzw. $240 \mathrm{~K}$ (Filme) zu $206 \mathrm{~K}$ (Abbildung 5.21) aufweist. Am zweiten Teil mit Iridium als Kontaktschicht werden STM und STS - Messungen durchgeführt. Zwei topographische Bilder sind in Abbildung 5.18 dargestellt. Das in (a) dargestellte Bild besitzt einen Scanbereich von $500 \times 500 \mathrm{~nm}^{2}$ und weist eine RMS - Rauhigkeit von 2,8 nm auf. Der Anstieg der Rauhigkeit im Vergleich zu den bisher diskutierten Messungen ist auf die erhöhte Schichtdicke zurückzuführen. Alle im Folgenden diskutierten und dargestellten Leitwertkarten basieren auf einem Scanbereich von $155 \times 155 \mathrm{~nm}^{2}$. Dargestellt ist in Abbildung 5.18 (b) beispielhaft eine Aufnahme bei einer Messtemperatur von 239 K. Hintergrund der Wahl dieser Vergrößerung ist der geänderte Schichtwiderstand und die daraus resultierende Änderung in der Größe der elektronischen Domänen. Die Größe der leitfähigen Bereiche fällt hier bei tieferen Temperaturen kleiner aus als bei den Schichten aus den vorherigen Kapiteln. Dementsprechend ist es zur Durchführung von Simulationen vernünftig, diesen verkleinerten Scanbereich zu wählen. Vergleicht man die Tieftemperaturmessung aus Kapitel 5.2 an LCMO auf $\mathrm{MgO}$ bei $78 \mathrm{~K}$ mit dem Ergebnis von dieser Probe aufgenommen bei $76 \mathrm{~K}$ (dargestellt in Abbildung 5.19-1) wird dieser Unterschied ersichtlich. Beide Messungen sind in der Tieftemperaturphase weit unterhalb des MIT durchgeführt worden; die mittleren Leitwerte unterscheiden sich nur minimal $\left(L_{78 \mathrm{~K}}=2,5 \cdot 10^{-2} \mathrm{nA} / \mathrm{V} \mathrm{zu} L_{76 \mathrm{~K}}=2,3 \cdot 10^{-2} \mathrm{nA} / \mathrm{V}\right)$. Stellt man jedoch einen Vergleich des prozentualen Anteils der metallisch verlaufenden Kennlinien beider Darstellungen an, zeigt sich dieser Unterschied: für den Scanbereich von $(118 \mathrm{~nm})^{2}$ aus Kapitel 5.2 ergibt sich ein Wert von $81 \%$; bei der Messung der aktuellen Schicht $(155 \mathrm{~nm})^{2}$ beläuft sich der Prozentsatz der metallisch verlaufenden Kennlinien aufgrund des Sauerstoffdefizits auf nur noch $37 \%$. 
a1)

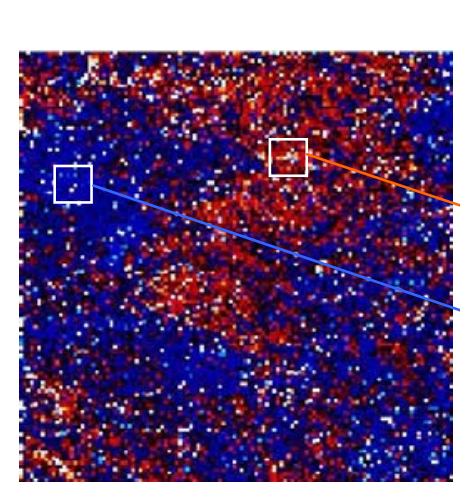

a2)

$\underline{76 \mathrm{~K}}$

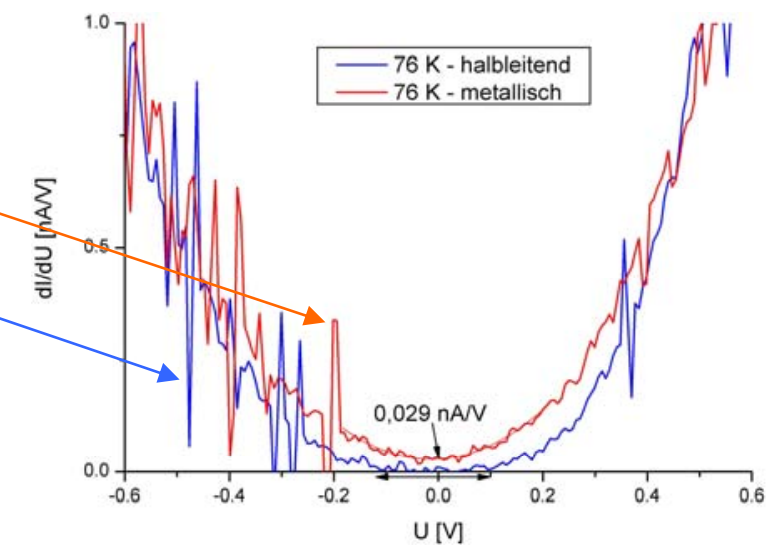

b2)

$\underline{114 K}$

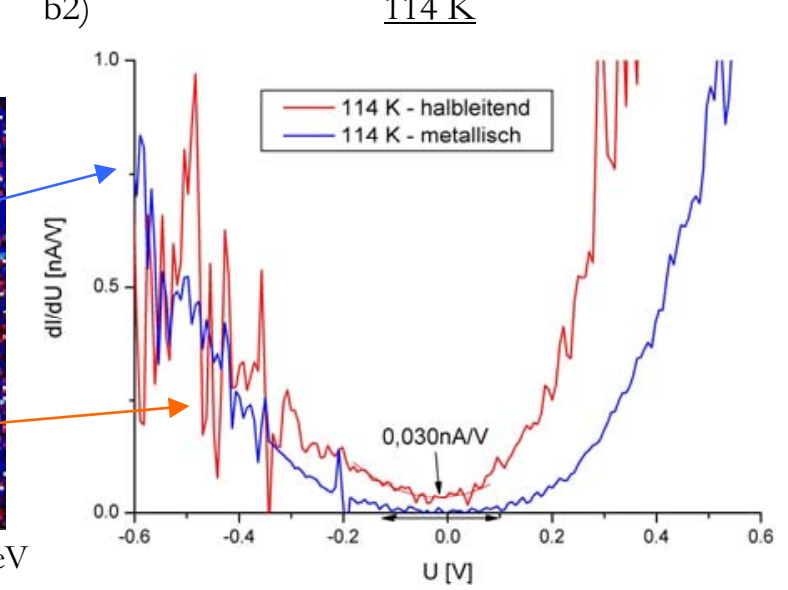

0

Gapbreite

$1 \mathrm{eV}$ $\underline{187 \mathrm{~K}}$

c2)

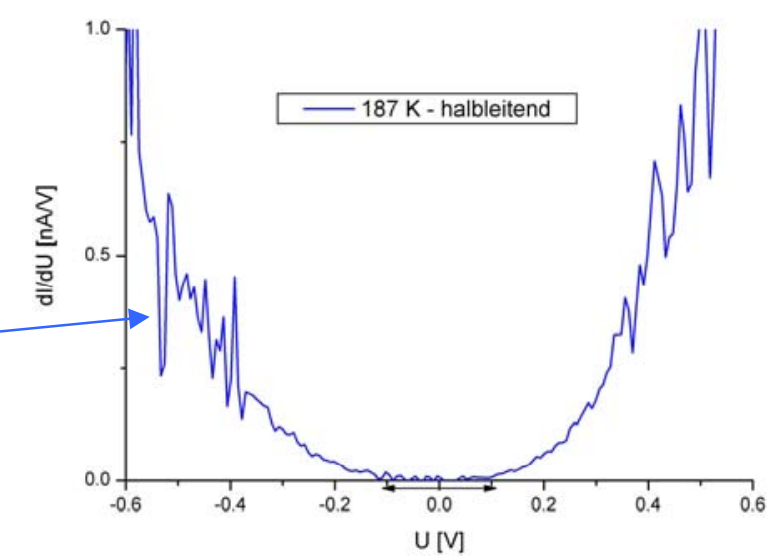

a3)

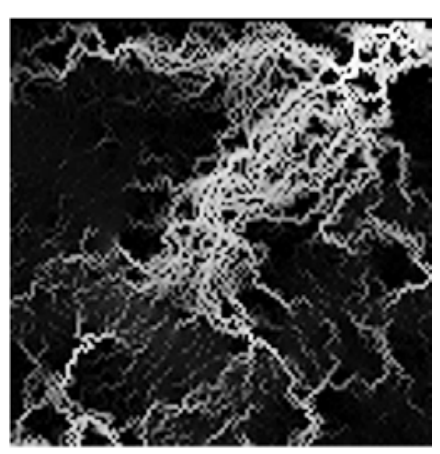

b3)

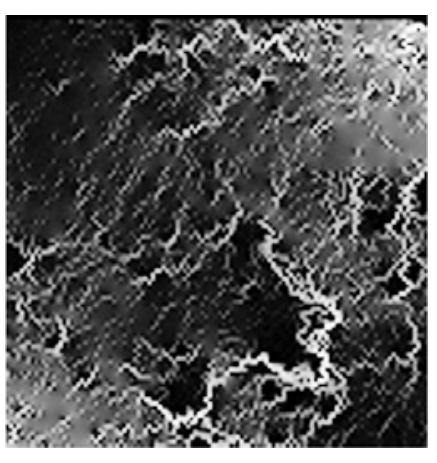

c3)

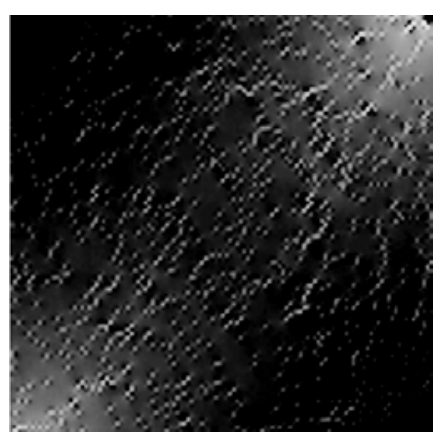

Abbildung 5.19-1: Spalte 1) Kombinierte Leitwert- und Bandlücken-Karten für verschiedene Temperaturen. $(155 \mathrm{~nm} \times 155 \mathrm{~nm})$

Spalte 2) Ableitungen von je $10 \times 10$ gemessenen Kennlinien (siehe 1. Spalte). Rot dargestellt sind die Ableitungen aus metallischen Domänen; blaue Ableitungen stammen von isolierenden Bereichen. Der Pfeil unterhalb der $\mathrm{x}$-Achse deutet das Ergebnis der automatisierten Kennlinienanalyse für die Energielücke an.

Spalte 3) Stromverlaufsbilder entsprechend der Karten aus der 1. Spalte 
d1)

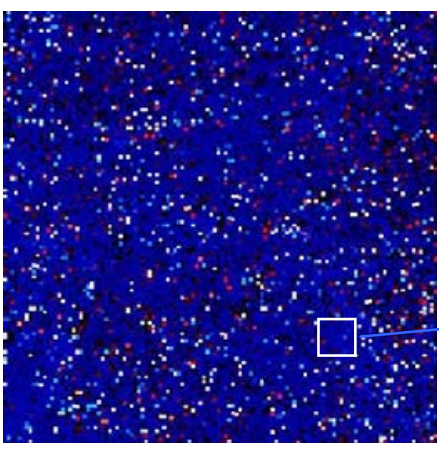

d2)

$\underline{214 K}$

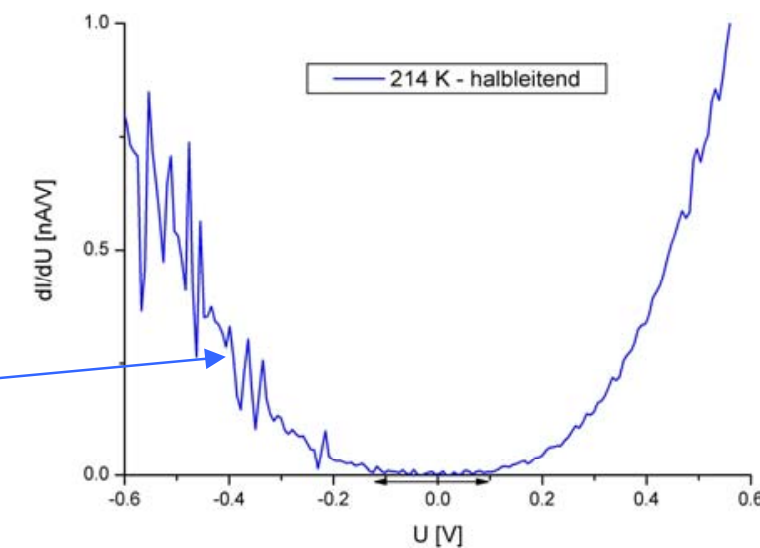

e1)

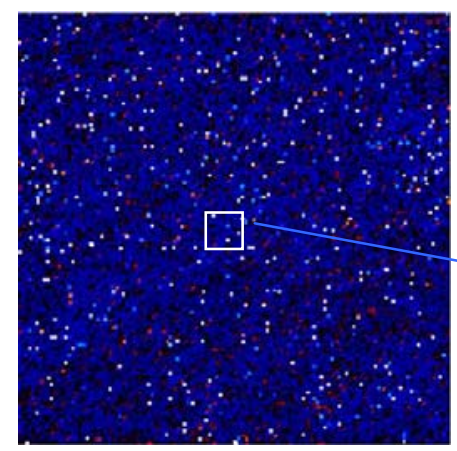

0

Gapbreite

$1 \mathrm{eV}$

e2)

$258 \mathrm{~K}$

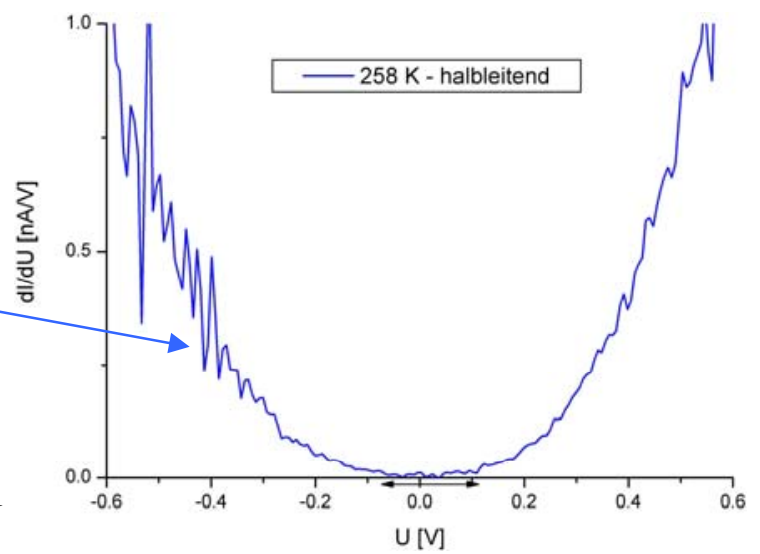

0 Leitfähigkeit $\quad 0,6 \mathrm{nA} / \mathrm{V}$

f1)

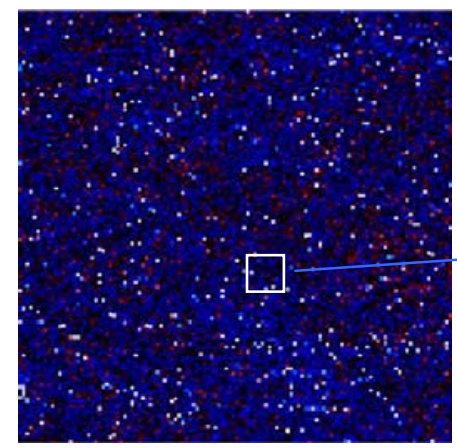

f2)
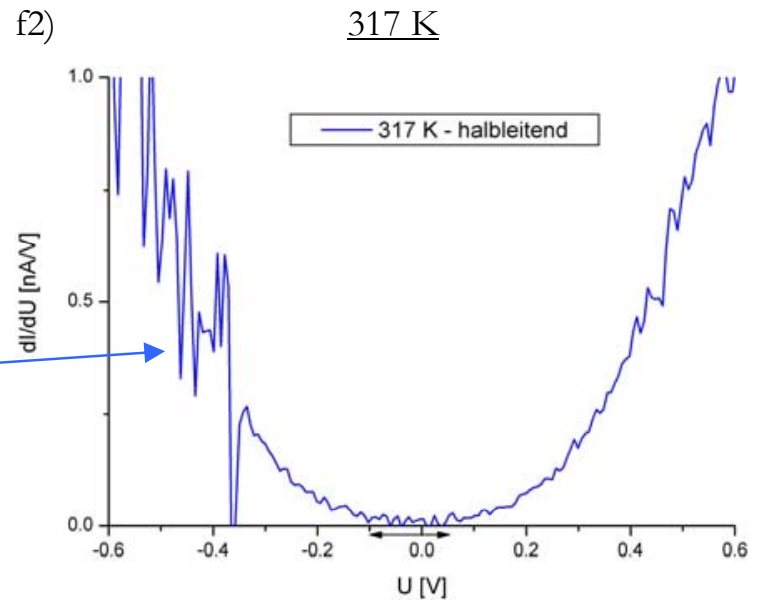

d3)

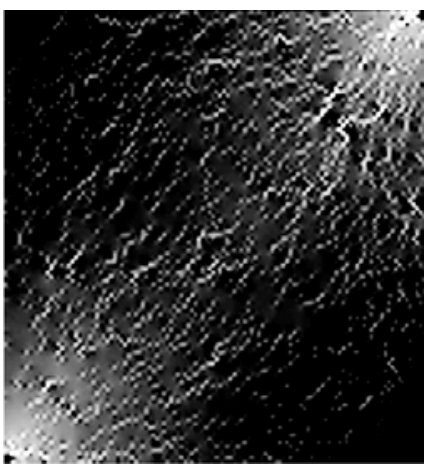

e3)

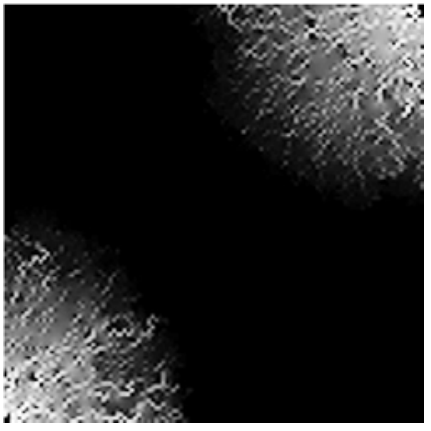

f3)

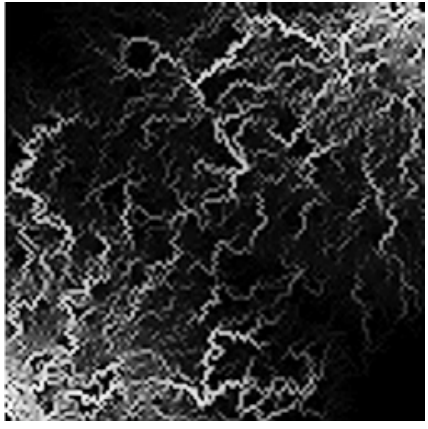

Abbildung 5.19-2: Spalte 1) Kombinierte Leitwert- und Bandlücken-Karten für verschiedene Temperaturen. $(155 \mathrm{~nm} \times 155 \mathrm{~nm})$

Spalte 2) Ableitungen von je $10 \times 10$ gemessenen Kennlinien (siehe 1. Spalte). Blau dargestellt sind die Ableitungen aus isolierenden Bereichen. Der Pfeil unterhalb der $\mathrm{x}-$ Achse deutet das Ergebnis der automatisierten Kennlinienanalyse für die Energielücke an.

Spalte 3) Stromverlaufsbilder entsprechend der Karten aus der 1. Spalte 
In Abbildung 5.19-1+2 sind an dieser sauerstoffreduzierten Probe temperaturabhängige Ergebnisse dargestellt. Die erste Spalte zeigt in einem Bereich von $155 \mathrm{~nm} \times 155 \mathrm{~nm}$ die kombinierten Leitwert- und Gapverteilungskarten. Rote Farben stellen metallisch leitfähige Bereiche dar. Liegt eine Kennlinie vor, die den Verlauf entsprechend eines Halbleiters aufweist, ist die Breite der ausgewerteten Bandlücke als Blauwert codiert. Bei $76 \mathrm{~K}$ sind deutlich leitfähige von isolierenden Domänen getrennt. Mit zunehmender Temperatur schrumpfen die metallischen Bereiche, bis schließlich bei $214 \mathrm{~K}$ (etwas oberhalb des MIT) nur noch vereinzelt kleine rote Flächen zu erkennen sind. Im gleichen Maß wachsen mit zunehmender Temperatur die isolierenden Bereiche. Dies spiegelt die Ergebnisse der vorherigen Kapitel wider. Nun ist aber besonders das Verhalten oberhalb des Übergangs von Interesse. Ein Vergleich der Karten von $214 \mathrm{~K}$ bis $317 \mathrm{~K}$ zeigt, dass die homogene blaue Färbung langsam abnimmt, die roten Flächen schwach zunehmen und bei $317 \mathrm{~K}$ deutlich sichtbar vorliegen. Infolgedessen zeigt die Analyse, dass die Breite der Bandlücke mit zunehmender Temperatur etwas abgenommen hat. Um dieses Ergebnis zu kontrollieren, sind in der zweiten Spalte die Ableitungen der Originaldaten entsprechend dem dargestellten Quadrat von $10 \times 10$ Kennlinien dargestellt. Die aus metallischen (halbleitenden) Bereichen stammenden Ableitungen sind rot (blau) aufgetragen. Bei $76 \mathrm{~K}$ und $114 \mathrm{~K}$ liegen genügend zusammenhängende leitfähige Kennlinien vor, so dass eine Analyse überhaupt möglich ist. Um das Minimum des Graphen bestimmen zu können, ist um die Werte nahe $0 V$ eine Parabel gefittet worden [78]. Das Minimum dieser Parabel ergibt sich bei $76 \mathrm{~K} z u$ 2,9 $10^{-2} \mathrm{nA} / \mathrm{V}$ und bei $114 \mathrm{~K}$ zu 3,0 $\cdot 10^{-2} \mathrm{nA} / \mathrm{V}$. Ein Vergleich mit den automatisiert bestimmten Werten (76 K: $L_{76 \mathrm{~K}}=2,3 \cdot 10^{-2} \mathrm{nA} / \mathrm{V}$ und $114 \mathrm{~K}: L_{114 \mathrm{~K}}=2,6 \cdot 10^{-2} \mathrm{nA} / \mathrm{V}$ ) zeigt eine gute Übereinstimmung. Der etwas unterschiedliche Verlauf der Ableitung bei $114 \mathrm{~K}$ (zwischen $0 \mathrm{~V}$ bis $0,4 \mathrm{~V})$ ist auf fehlerhafte Kennlinien ${ }^{26}$ in diesem Bereich zurückzuführen uns soll nicht weiter diskutiert werden. Die blau dargestellten, gemittelten Ableitungen zeigen bei allen Temperaturen nahe $O V$ ein Gap mit unterschiedlicher Breite. Um einen direkten Vergleich mit den Ergebnissen der automatisierten Kennlinienanalyse durchführen zu können, ist der dort bestimmte Wert als schwarzer Pfeil unterhalb der $\mathrm{x}$ - Achse eingetragen worden und zeigt eindeutig eine gute Korrelation zu den rein experimentellen Daten. Zur Bestimmung dieser Werte ist die Gapbreite der einzelnen Messungen in einer Häufigkeitsverteilung aufgetragen worden. Abbildung 5.20 (a) zeigt die beiden Histogramme für die Messungen bei $317 \mathrm{~K}$ und $76 \mathrm{~K}$. Beide Graphen sind einem Gaußfit unterzogen worden, wobei sich die jeweiligen Maxima zu 0,22 eV (76 K) und 0,16 eV $(317 \mathrm{~K})$ bestimmen lassen.

\footnotetext{
${ }^{26}$ Weiße Punkte in den kombinierten Leitwert- und Isolationskarten weisen auf fehlerhafte Kennlinien hin. Die Analysesoftware ist dort nicht im Stande, einen ordnungsgemäßen Fit durchzuführen um die Gapbreite bzw. die Leitfähigkeit zu bestimmen. Die Software erteilt diesen Punkten automatisch den Maximalwert.
} 
a)

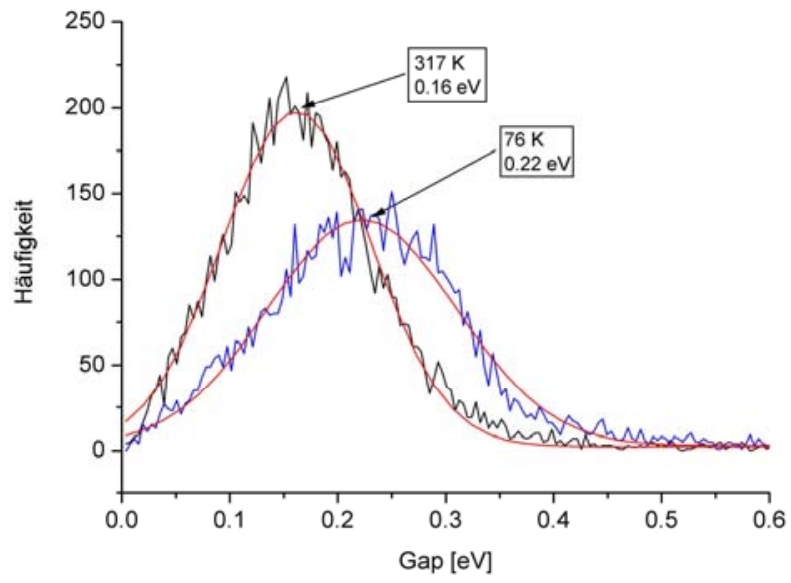

b)
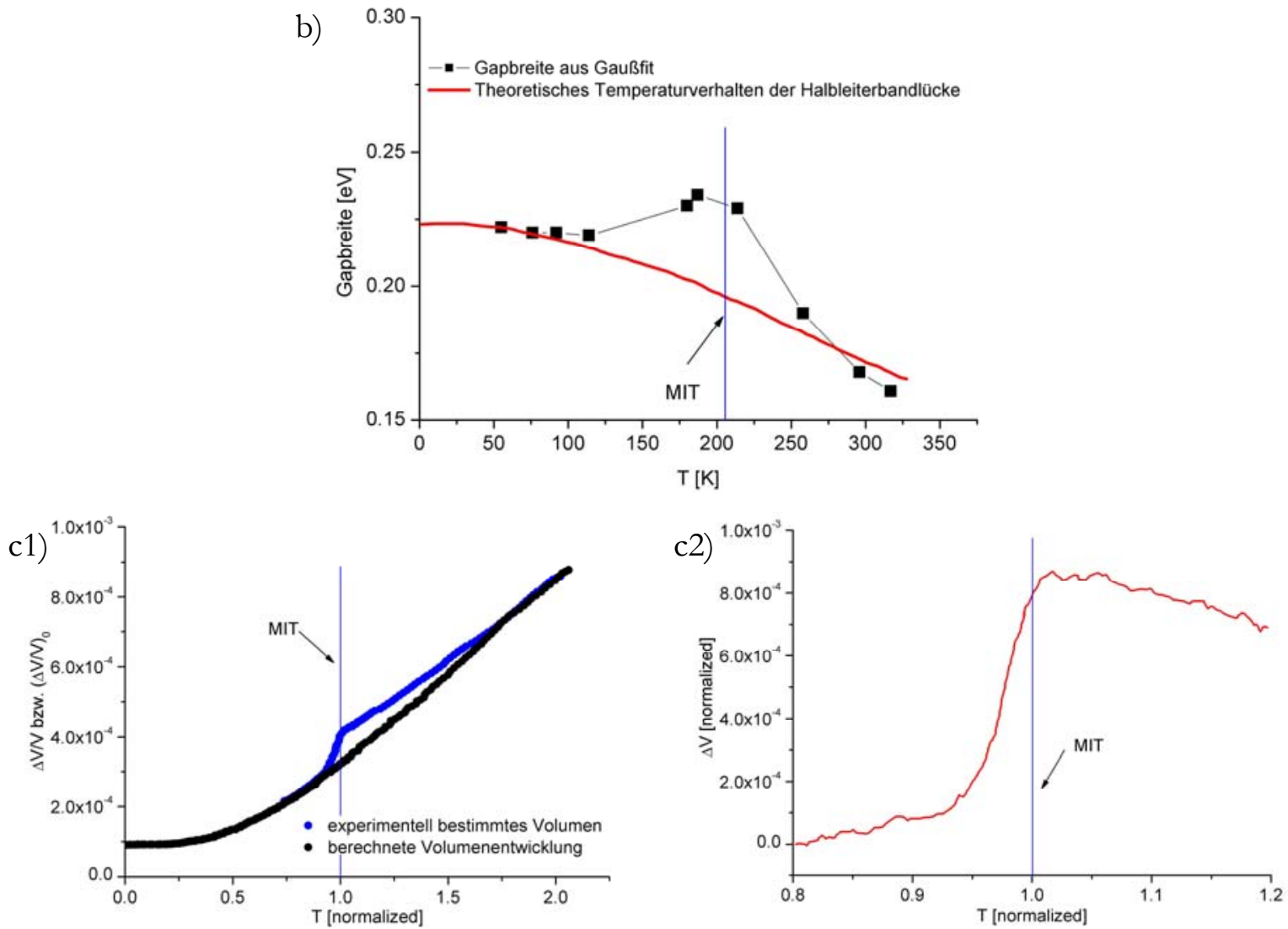

Abbildung 5.20: a) Häufigkeitsverteilungen der durch die Analysesoftware bestimmten Gapbreiten für $76 \mathrm{~K}$ und $317 \mathrm{~K}$. Das Maximum ergibt sich aus einem Gaußfit bei $76 \mathrm{Kzu} 0.22 \mathrm{eV}$ und bei $317 \mathrm{Kzu} 0.16 \mathrm{eV}$.

b) Die schwarzen Rechtecke stellen die entsprechend in (a) bestimmten temperaturabhängigen Gapbreiten dar. Rot dargestellt ist die für einen Halbleiter typische Änderung der Gapbreites [79]. Die blaue Linie weist auf die Temperatur des Metall - Isolator - Übergang hin.

c) Die Differenz aus dem experimentell best. Volumen an LCMO (blau (c1)) und der berechneten Volumenänderung nach Grüneisen (schwarz (c1)) ergibt die temperaturabhängige Volumenänderung (rot (c2)) [80]. 
In Abbildung 5.20 (a) ist deutlich zu erkennen, dass bei Temperaturerhöhung eine Verschiebung des Kurvenverlaufs bzw. des Maximums zu kleineren Werten hin stattfindet. Trägt man die Ergebnisse der mittleren Gapbreite in einem Diagramm für jede Temperatur auf, wird diese Entwicklung ersichtlich (schwarze Rechtecke in Abbildung 5.20 (b)). Dies unterstreicht die oben genannte Feststellung, dass sich die gemessene Gapbreite mit zunehmender Temperatur ändert. Generell kann die Abnahme der Gapbreite zum Teil auf die bei Halbleitern bekannte intrinsische Leitfähigkeit zurückgeführt werden. Hierbei besitzen Elektronen innerhalb des Valenzbandes bei einer von $T=0 \mathrm{~K}$ verschiedenen Temperatur die Möglichkeit, in das Leitungsband zu gelangen und somit Eigenleitfähigkeit zu erzeugen. Es handelt sich um einen temperaturinduzierten Prozess, d.h. mit steigender Temperatur nimmt die Leitfähigkeit zu und die Gapbreite ab. Ein direkter Vergleich mit Literaturwerten [79; 81] zeigt eine gute Übereinstimmung der Größenordnung der Gapbreitenabnahme von tiefer Temperatur (nahe $0 \mathrm{~K}$ ) zu $300 \mathrm{~K}$ :

\begin{tabular}{|c|c|c|}
\hline Material & $\mathbf{E}_{\mathrm{g}}[\mathbf{e V}] \mathbf{( T}=\mathbf{0} \mathbf{K}$ bzw. 55 K bei LCMO) & $\left.\mathbf{E}_{\mathbf{g}}[\mathbf{e V}] \mathbf{( T = 3 0 0 ~ K}\right)$ \\
\hline LCMO & 0.22 & 0.16 \\
\hline InSb[79; 81] & 0.23 & 0.16 \\
\hline InAs[79; 81] & 0.43 & 0.35 \\
\hline
\end{tabular}

Ein direkter Vergleich des experimentell bestimmten Kurvenverlaufs mit den theoretischen Werten in Abbildung 5.20 (a) zeigt in der Nähe des Metall - Isolator - Übergangs (blau markiert) ein unterschiedliches Verhalten: Die Bandlücke nimmt nicht gleichmäßig ab, sondern besitzt an dieser Stelle ein deutliches Maximum. Mögliches Erklärungspotential kann hierzu die von De Teresa [80; 82] gemessene Volumenänderung bei LCMO am Übergang liefern. Diese ist das Ergebnis der Gitterverzerrung, die aus der Bildung und Vernetzung der isolierenden Phase resultiert. In Abbildung 5.20 (c) ist diese Volumenänderung dargestellt [80] und verdeutlicht eine Volumenzunahme nahe des MIT, die auf einen strukturellen Phasenübergang hindeutet. Diese verzerrungsbedingte Vergrößerung des Volumens hat Einfluss auf die Bindungslängen der Atome und somit auch auf die Größe der Bandlücke [83; 84]. Dadurch, dass die Manganat Atome ihren gegenseitigen Abstand vergrößern, ändern die Orbitale ihre Positionen zueinander und die Gapbreite erhöht sich.

In der dritten Spalte aus Abbildung 5.19-1+2 sind die bei der Simulation entstandenen Strompfadkarten dargestellt. Bei Temperaturen weit unterhalb des Metall - Isolator Übergangs zeigt sich in den metallischen Domänen ein feinmaschiges Netz aus stromdurchflossenen Bereichen, die stellenweise durch isolierende Domänen abgegrenzt werden. An diesen Stellen können sich Pfade ausbilden, die eine erhöhte Stromdichte aufweisen. Im Vergleich zu den 
Simulationen an vorhergehenden Messungen dieser Arbeit zeigt sich, dass sich hier die Perkolationspfade sehr viel früher ausbilden, schon bei $76 \mathrm{~K}$ sind diese ausgeprägt vorhanden. Als Hauptgrund hierfür ist die oben diskutierte Sauerstoffreduktion anzuführen. Die maximale Stromdichte ist in allen Auftragungen auf den jeweils maximalen Wert normiert. Somit kann nur der strukturelle Verlauf beschrieben werden, eine Aussage über den bestimmten Widerstand kann nicht getroffen werden. Diese sind aus Abbildung $5.21 \mathrm{zu}$ entnehmen. Bei $187 \mathrm{~K}$ nimmt der Widerstand stark zu, wobei in der Karte nur noch eine homogene Stromverteilung über schlecht leitende Bereiche zu verzeichnen ist. Die Strompfadbildung ist zusammengebrochen - Es liegt keine Perkolation mehr vor. Ein vergleichbares Bild zeigt sich bei der Betrachtung der Auftragung knapp oberhalb des MIT bei $214 \mathrm{~K}$. Sobald man sich etwas entfernt vom MIT bei höheren Temperaturen bewegt, beginnt sich der Strom nun homogen verteilt in die Probe zu bewegen. Die Abbildung bei $258 \mathrm{~K}$ zeigt die Anfangsstadien dieses Vorganges. Dieses Verhalten ist auf das Vorhandensein einer recht schlecht leitenden Phase zurückzuführen, die sich in den Leitwert- und Gapverteilungen als dunkelrot bis lilafarbener Hintergrundton bemerkbar macht und mit steigender Temperatur langsam an Intensität gewinnt.

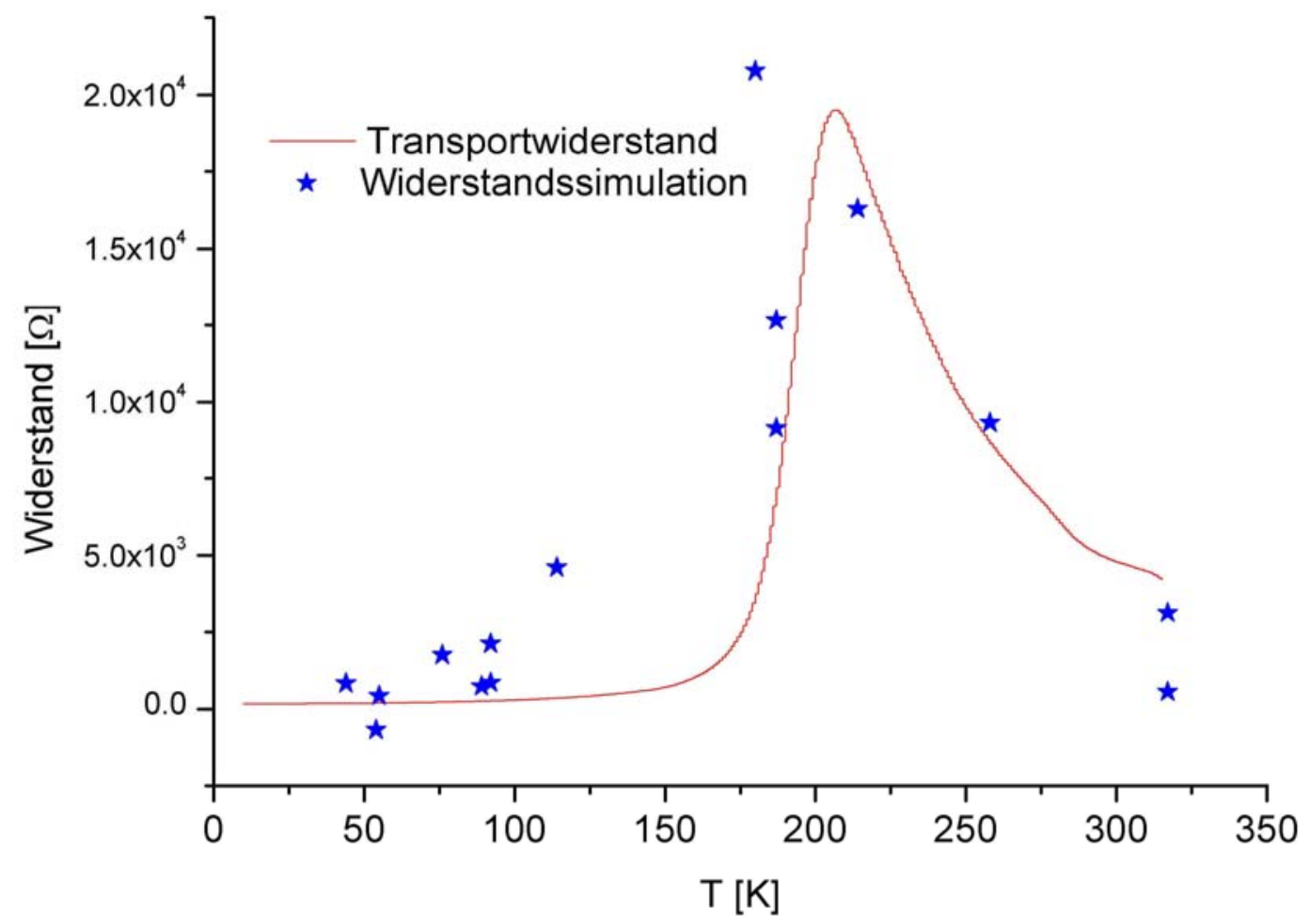

Abbildung 5.21: Transportwiderstand (rote Linie) und normierte Netzwerkwiderstände der Simulation (blaue Sterne). 
Die oben schon angesprochene Abbildung 5.21 stellt einen Vergleich des gemessenen Transportwiderstandes mit den normierten Ergebnissen der Widerstandsnetzwerksimulationen dar. Der Verlauf der simulierten Widerstände beschreibt recht gut den des gemessenen Transportwiderstandes. Unterhalb des MIT weisen die Ergebnisse der Simulation im Vergleich zur Transportmessung einen höheren Widerstandswert auf. Bei Transportmessungen breiten sich die Strompfade im bulk dreidimensional aus. Daraus lässt sich auf eine langsamer abnehmende Leitfähigkeit als im 2 - Dimensionalen schließen. Der Bereich oberhalb des Übergangs, der in den vorhergehenden Messungen nicht betrachtet werden konnte, zeigt nun eine gute Übereinstimmung von Transportmessung und Simulation. 


\section{Abschließende Diskussion}

Im Kapitel 5 sind Messungen und Ergebnisse an LCMO und LSMO bei variablen Temperaturen vorgestellt worden, dabei zeigt sich deutlich eine Phasenseparation bei Temperaturen unterhalb des Metall - Isolator - Übergangs in isolierende und leitfähige Domänen. Bei sehr tiefen Temperaturen ist hauptsächlich die leitfähige Phase anzutreffen, demgegenüber liegt in der Nähe des MIT fast ausschließlich isolierendes Verhalten vor, wobei nur noch wenige und kleine leitfähige Domänen zu finden sind. In Abbildung 6.1 ist ein hochaufgelöstes STM - Bild einer LCMO - Schicht 3 dimensional dargestellt, wobei die Topographie mit den Ergebnissen der STS - Analyse kombiniert aufgetragen sind. Die Messung wurde unterhalb des MIT's bei $T=168 \mathrm{~K}$ durchgeführt und zeigt voneinander getrennt vorliegende metallische (rot dargestellt) und isolierende Bereiche (schwarz), die nicht direkt mit der Kornstruktur korrelieren.

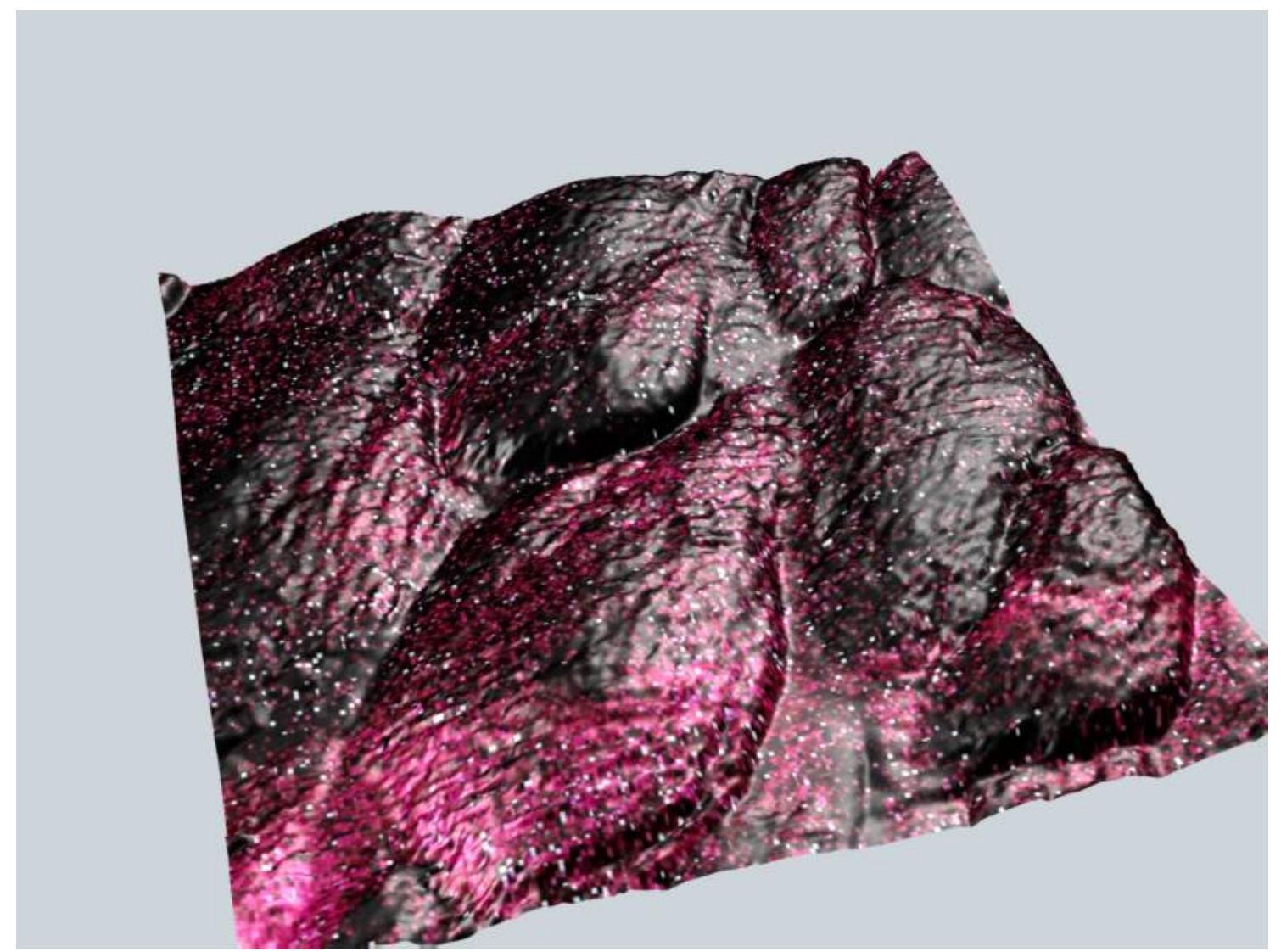

Abbildung 6.1: Hochaufgelöste STM - Topographie mit STS - Leitfähigkeiten an einer $200 \mathrm{~nm}$ dicken LCMO - Schicht auf MgO bei $186 K$. Dargestellt ist ein Bereich von $168 \mathrm{~nm} \times 168 \mathrm{~nm}$ mit einem maximalen Höhenunterschied von $10 \mathrm{~nm}$. Rot (schwarz) dargestellte Bereiche besitzen metallisch leitfähiges (isolierendes) Verhalten. 
Um eine Aussage über den Zusammenhang zwischen Domänengröße und Temperatur treffen zu können, wurde eine Analyse der Daten mittels Autokorrelationsfunktionen [85] durchgeführt ${ }^{27}$. Ein Beispiel und die Ergebnisse für die Messungen an LSMO auf MgO (Kapitel 5.4) und LSMO auf Iridium (Kapitel 5.5) sind in Abbildung 6.2 dargestellt.
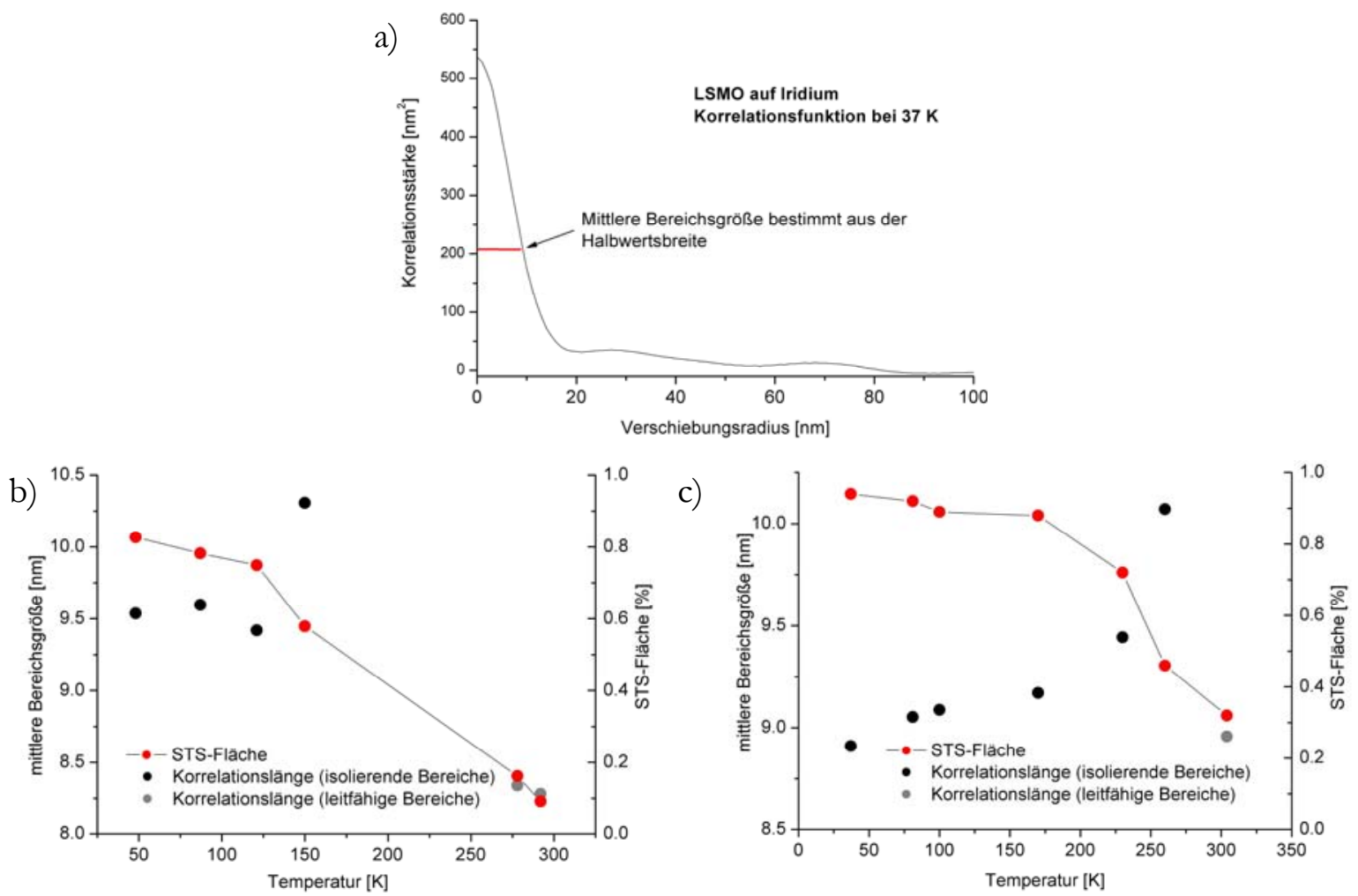

Abbildung 6.2: Bestimmung der mittleren Bereichsgröße aus der Halbwertsbreite (rot eingezeichnet) beispielhaft dargestellt für LSMO auf Iridium bei $37 \mathrm{~K}$ (a). Temperaturabhängige Ergebnisse der Autokorrelationsfunktion für (b) LSMO auf MgO und (c) LSMO auf Iridium (schwarze und graue Kreise). Rot gekennzeichnet sind die unnormierten, bestimmten Flächenanteile der leitfähigen Domänen dargestellt.

Die beiden Graphen in Abbildung 6.2 (b) und (c) zeigen bei tiefen Temperaturen nur eine schwache Änderung der mittleren Bereichsgröße für die isolierenden Domänen. Die typische Domänengröße liegt in (b) bei $9,5 \mathrm{~nm}(48 \mathrm{~K}-121 \mathrm{~K})$ und ergibt sich in (c) zu $9.1 \mathrm{~nm}(37 \mathrm{~K}-$ 170 K). Vergleichbar nimmt der Flächenanteil der leitfähigen Bereiche in beiden Systemen nur schwach ab (rote Kreise). Erst bei einer verstärkten Abnahme der metallisch leitfähigen Bereichsfläche ist eine erhöhte Zunahme der isolierenden Bereichsgröße aufgrund von vernetzender Koaleszenz festzustellen. Oberhalb eines Flächenanteilswertes von ca. $50 \%$, bei

\footnotetext{
${ }^{27}$ Die verwendete Software [85] ist zur Analyse von Kornwachstum entwickelt worden. Dementsprechend ist bei Ausgangsdaten, die einen sehr hohen Kontrast und zunehmende Vernetzungen zeigen, mit Abweichungen zu rechnen. Die Ergebnisse besitzen jedoch eine sehr gute Konsistenz und werden deshalb zur Diskussion herangezogen.
} 
dem isolierende und leitfähige Domänen im gleichen Maße existieren, liegen leitfähige Domänen innerhalb einer isolierenden Matrix vor - dementsprechend zeigt die Korrelationsfunktion niedrigere Werte an (graue Kreise). Ein Vergleich der beiden Graphen aus Abbildung 6.2 zeigt, dass beide einen vergleichbaren Verlauf besitzen, welches nur bei verschiedenen Temperaturen eintritt. Der LSMO - Film auf dem Iridiumsubstrat zeigt im Vergleich erst bei höheren Temperaturen eine wachsende Vernetzung der isolierenden Domänen - ein Ergebnis, dass sich auch bei der Betrachtung der Leitfähigkeitsverteilungen aus Abbildung 5.11 und Abbildung 5.16 ergibt.

Ähnliche Resultate bezüglich der Phasenseparation an $\mathrm{La}_{0.7} \mathrm{Ca}_{0.3} \mathrm{MnO}_{3}$ sind von Fäth [10] vorgestellt worden. Es handelt sich bei seinen Ergebnissen um magnetfeldabhängige STS Messungen an einem Einkristall unterhalb des MIT, welche in Abbildung 6.3 dargestellt sind.

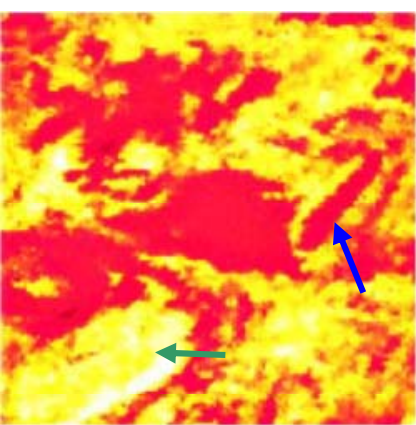

$0 \mathrm{~T}$

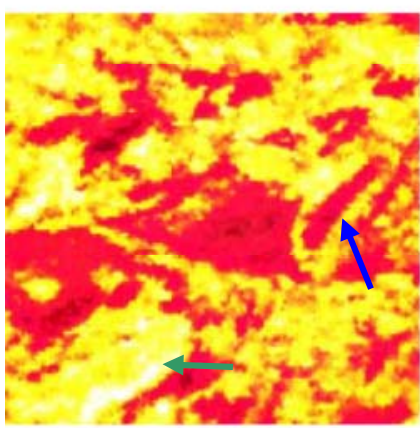

$0.3 \mathrm{~T}$

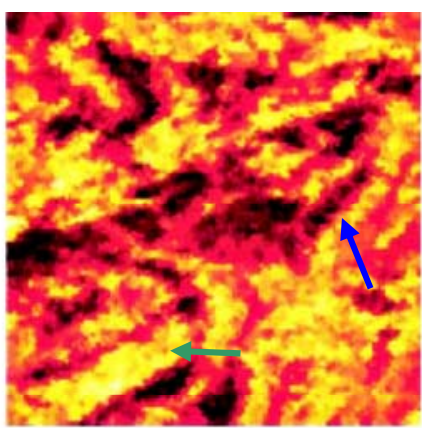

$1 \mathrm{~T}$

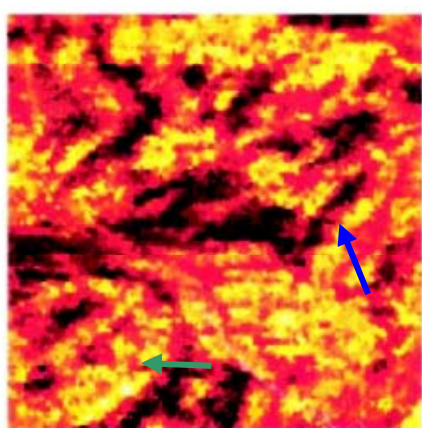

$3 \mathrm{~T}$

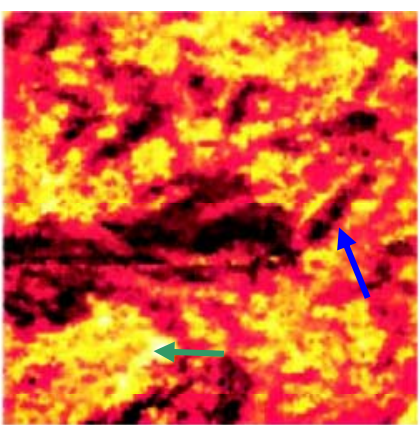

$5 \mathrm{~T}$

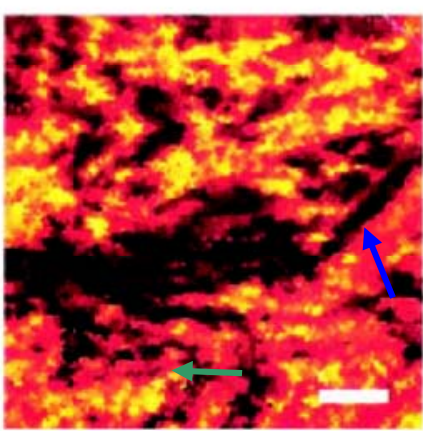

$9 \mathrm{~T}$

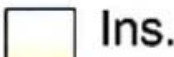

Met.

Abbildung 6.3: Magnetfeld abhängige STS-Messungen an einem LCMO-Einkristall bei einer Temperatur unterhalb des Metall-Isolator - Übergangs mit einer Größe von jeweils $610 \mathrm{~nm} \times 610 \mathrm{~nm}$. Helle (dunkle) Farben stellen isolierende (metallische) Bereiche dar $[10]$.

Aufgetragen sind jeweils Messbereiche von $610 \mathrm{~nm} \times 610 \mathrm{~nm}$, die sich im angelegten Magnetfeld als veränderlicher Parameter unterscheiden. Helle Farbwerte signalisieren ein isolierendes Verhalten der Oberfläche, wohingegen dunkel dargestellte Bereiche als metallisch zu klassifizieren sind. Dieser Auftrennung liegen STS - Kennlinien zugrunde, die im Unterschied zu 
den Ergebnissen aus dieser Arbeit bei $U=3 V$ und nicht bei $U=0 V$ analysiert wurden. Ein Vergleich aller Messungen zeigt, dass sich Teilbereiche der elektronischen Struktur durch Anlegen des Feldes kaum ändern (siehe blauer Pfeil), einzig ihre Leitfähigkeit nimmt zu. Es existieren aber auch Bereiche, innerhalb derer mit zunehmendem Magnetfeld Fluktuationen eintreten (grüne Pfeile). Generell zeigen alle Messungen ein feldabhängiges Verhalten, wobei mit wachsendem Magnetfeld ein zunehmendes metallisches Verhalten der Probenoberfläche zu beobachten ist. Zusammenfassend können folgende Aussagen getroffen werden:

Die mittlere Bereichsgröße wird von Fäth [10] mit ca. $10 \mathrm{~nm}$ angegeben und ist somit konsistent mit den oben genannten Ergebnissen der temperaturabhängigen Messreihen.

Die im Rahmen dieser Arbeit durchgeführten Messungen an dem LSMO - Einkristall (Kapitel 5.3) zeigen entsprechend den Ergebnissen von Fäth ein inhomogenes elektronisches Verhalten bei Temperaturen unterhalb des MIT.

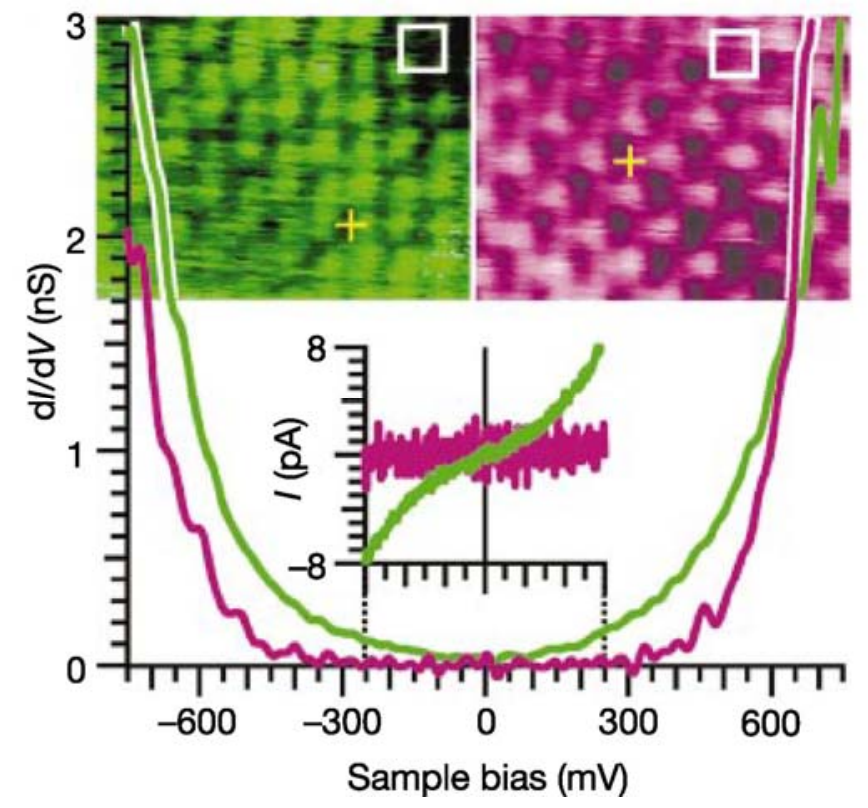

Abbildung 6.4: STM - und STS-Messungen (je 3,7 $\mathrm{nm} \times 2,9 \mathrm{~nm}$ ) bei Raumtemperatur an einem $\mathrm{Bi}_{0.24} \mathrm{Ca}_{0.76} \mathrm{MnO}_{3}-$ Einkristall [11]. Grün dargestellt sind Bereiche mit metallisch verlaufenden Kennlinien - rote Flächen zeigen Isolierenden Charakter .

Aber nicht nur die Manganate vom Typ LCMO und LSMO zeigen eine auftretende Phasenseparation in isolierende und leitfähige Domänen. STM - und STS - Messungen von Renner [11] liefern vergleichbare Ergebnisse an einem $\mathrm{Bi}_{0.24} \mathrm{Ca}_{0.76} \mathrm{MnO}_{3}$-Einkristall. Im Unterschied zu den oben genannten Manganaten liegen bei hohen Temperaturen die $\mathrm{Mn}^{3+}$ - und $\mathrm{Mn}^{4+}$-Ionen statistisch verteilt vor, wodurch sich ein leitfähiges Grundsystem einstellt. Wird die Temperatur reduziert, beginnen sich die Kationen zu ordnen, und es entsteht eine isolierende, 
ladungsgeordnete ${ }^{28}$ Phase, begleitet von einer leichten Zunahme der Gitterabstände [86]. In Abbildung 6.4 sind zwei bei Raumtemperatur aufgenommen STM-Bilder mit atomarer Auflösung dargestellt. Darunter befinden sich zu jedem Bild eine Kennlinie und deren Ableitung, die an den Positionen der gelben Kreuze in den Bildern der jeweiligen Farbe aufgenommen worden sind. Der Verlauf der dargestellten Kennlinien und Ableitungen entspricht denen, die in Kapitel 5 an LCMO gemessen wurden (vgl. Abbildung 5.4 (c) und Abbildung 5.19). Metallisch leitfähige Bereiche zeigen Kennlinien mit einer vorhandenen Steigung, wohingegen aus den Ableitungen der Kennlinien isolierender Domänen die Gap - Breite bestimmt werden kann. Dementsprechend handelt es sich bei dem linken Bild (grün dargestellt) um einen Ausschnitt einer metallischen Domäne und bei dem rechten (rot abgebildet) um einen isolierenden Teil der Probe. Ein direkter Vergleich der mittleren Leitfähigkeit $L_{B C M O}=0.02 n A / V$, die aus der Auftragung der metallisch verlaufenden Kennlinie errechnet werden kann, mit den Ergebnissen, die in dieser Arbeit bestimmt wurden, weist eine gute Übereinstimmung auf. Ein Vergleich der Gap - Breite mit den bestimmten Werten an LCMO und LSMO aus Kapitel 5 zeigt die gleiche Größenordnung auf, fällt aber mit $0.7 \mathrm{eV}$ bei diesem System etwas größer aus.

In Abbildung 6.5 (a) ist eine topographische STM - Aufnahme $(3,5 \mathrm{~nm} \times 3,5 \mathrm{~nm})$ von BCMO auf atomarer Skala dargestellt, die einen Bereich mit vorliegender Phasenseparation zeigt [11]. Eine Betrachtung der atomaren Abstände entlang der orange eingezeichneten Linie (aufgetragen unterhalb des Bildes) zeigt einen deutlichen Wechsel zwischen zwei verschiedenen Strukturen. Dementsprechend handelt es sich bei der im rechten, oberen Abschnitt vorliegenden Struktur um eine isolierende, CO - Domäne. Die gelb eingezeichnete, scharf verlaufende Grenze signalisiert den Wechsel zwischen den isolierenden und metallischen Domänen. Abbildung (b) zeigt einen 1,7 $\mathrm{nm}^{2}$ großen Bereich der CO - Phase, der bei einer Temperatur unterhalb des Übergangs aufgenommen wurde. Die vorliegende Struktur entspricht der isolierenden Domäne bei Raumtemperatur. Zusätzlich sind hier die Atompositionen von Mangan- und Sauerstoffionen eingezeichnet, um die Ladungsordnung der isolierenden Phase zu verdeutlichen.

Die bisher diskutierten Ergebnisse weisen auf eine vorliegende Phasenseparation bei festen Temperaturen von leitfähigen und isolierenden Domänen hin, wobei die jeweilige Ausdehnung im Bereich von einigen Nanometern anzusiedeln ist. Experimentelle und theoretische Arbeiten an weiteren Manganat - Typen zeigen ein vergleichbares Verhalten, wobei die Domänengrößen als teilweise mesoskopisch (z.B. $\sim 300 \mathrm{~nm}$ an einem $\mathrm{La}_{0.25} \mathrm{Pr}_{0.375} \mathrm{Ca}_{0.375} \mathrm{MnO}_{3}-$ Film [50]) oder kleiner (Tröpfchenmodell $\left.{ }^{29}[87 ; 88]\right)$ angenommen werden.

\footnotetext{
${ }^{28}$ Engl.: Charge Ordered (CO)

${ }^{29}$ Bei diesem Modell wird von einer flüssigkeitsähnlichen, räumlichen Verteilung von magnetischen „Tröpfchen“ ausgegangen.
} 

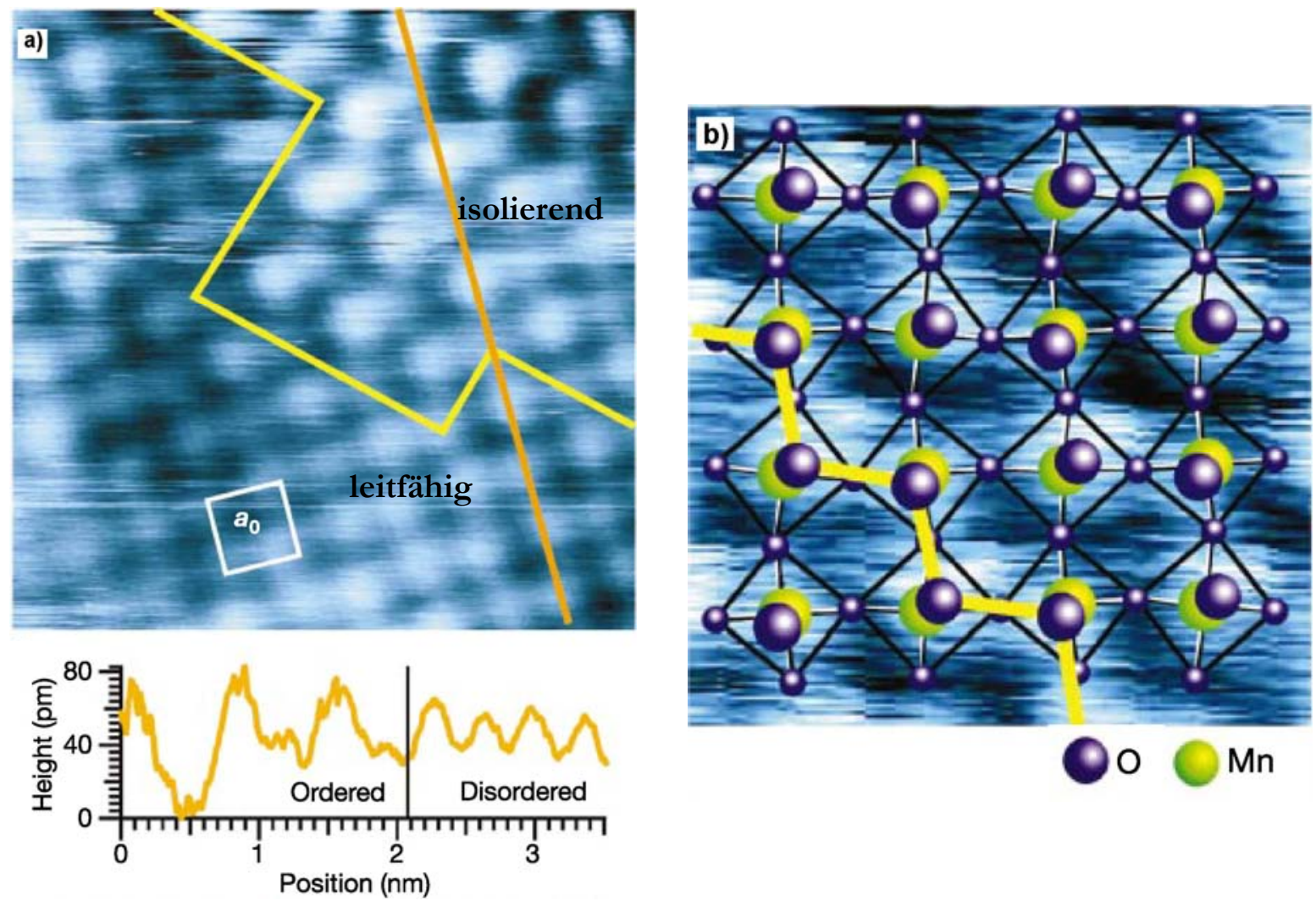

Abbildung 6.5: a) Atomare STM - Aufnahme an BCMO mit einer Größe von 3,5 nm × 3,5 nm. Es liegt eine Phasenseparation zwischen isolierenden und leitfähigen Bereichen vor (gelb dargestellt ist die Grenze zwischen Leitfähigen und isolierenden Domänen, orange eingezeichnet ist der Bereich, von dem das Höhenprofil (unter dem STM-Bild) stammt.

b) Tieftemperaturaufnahme unterhalb des Übergangs der isolierenden, CO - Struktur. Als lila- und grüngefärbte Kugeln sind die Atompositionen des Sauerstoffes und des Mangans eingezeichnet (beide aus [11]).

Von Interesse ist nun weiter, inwiefern die Phasenseparation Einfluss auf die Perkolation und auf den Metall - Isolator - Überhang hat. Eine mögliche Umsetzung liefert das hinzulegieren von Magnesium zu La ${ }_{0.67} \mathrm{Sr}_{0.33} \mathrm{Mn}_{1-\mathrm{x}} \mathrm{Mg}_{\mathrm{x}} \mathrm{O}_{3}$ [89]: Hintergrund hierfür ist, dass $\mathrm{Mg}^{2+}$ - Ionen den gleichen Radius wie die Mn - Ionen besitzen und somit keinen direkten Einfluss auf die Kristallstruktur des Manganates haben. Sie zeigen aber im Unterschied zum Manganion kein magnetisches Moment. Das Ziel ist hierbei, Einfluss auf die Magnetisierbarkeit der Probe zu erlangen, bzw. durch Erhöhen der $\mathrm{Mg}$-Konzentration in der Probe die Magnetisierung gezielt zu unterdrücken. Dadurch tritt eine Änderung des Volumenanteils der metallisch leitfähigen Bereiche ein und folglich auch eine Verschiebung der Temperatur des MIT.

In Abbildung 6.6 sind vier Transportmessungen für die Magnesiumkonzentrationen von $x_{a}=0 \%$, $x_{b}=5 \%, x_{c}=10 \%$ und $x_{d}=15 \%$ bzw. $20 \%$ dargestellt. Eindeutig ist eine Verringerung der 
Übergangstemperatur bei Zunahme der Konzentration festzustellen. Zusätzlich nimmt der Probenwiderstand systematisch zu. Aber nicht nur der Austausch der Kationen führt zu diesem Ergebnis, auch eine Variation der Seltene - Erd - bzw. der Erdalkali - Ionen liefert vergleichbare Resultate [90].
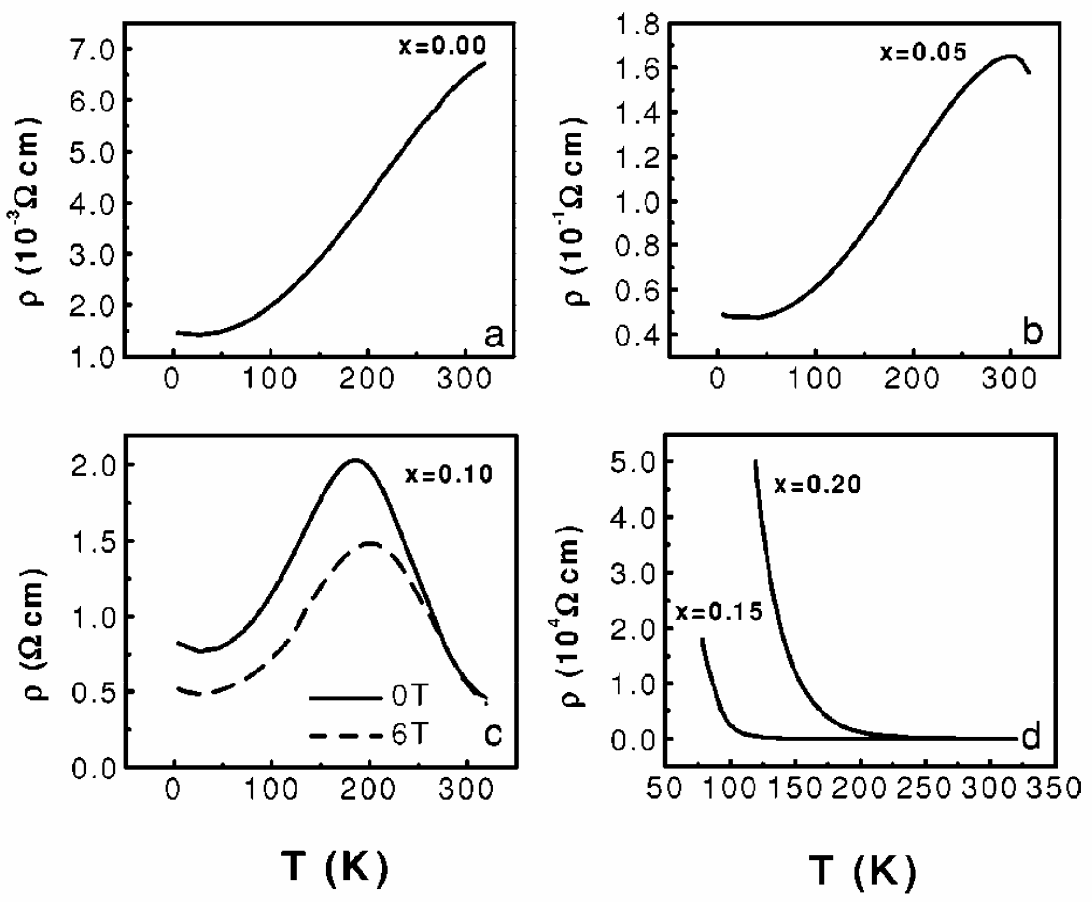

\footnotetext{
Abbildung 6.6: Änderung des Transportwiderstandes durch Änderung des Magnesiumanteils $x$ inLa ${ }_{0.67} \mathrm{Sr}_{0.33} \mathrm{Mn}_{1-\mathrm{x}} \mathrm{Mg}_{\mathrm{x}} \mathrm{O}_{3}$ [89].
}

Einen weiterführenden theoretischen Ansatz zur Phasenseparation und der daraus resultierenden Perkolation hat Burgy [7] bearbeitet. Als Ausgangspunkt sind die in Kapitel 2.4 vorgestellten theoretischen Grundlagen zu sehen. Burgy erweitert das dort beschriebene Ising-Modell um weitere Terme mit der Zielsetzung, nicht nur die Wechselwirkung benachbarter Spins zu betrachten. Somit ist es für ihn möglich, Monte - Carlo - Simulationen durchzuführen, bei denen auch Kopplungen zu diagonal gegenüberliegenden Nachbarn innerhalb einer Zelle des Gitters beeinflusst werden können. Ergebnisse der MC - Simulation bei fester Temperatur aber mit unterschiedlichen diagonalen Kopplungsparametern sind in Abbildung 6.7 (c1-3) dargestellt und besitzen je eine Auflösung von $64 \times 64$ Gitterpunkten. Hierbei wurden die Intervallgrenzen zu $W= \pm 1,5$ vorgegeben ${ }^{30}$. Sie bestimmen den Rahmen, in dem das chemische Potential $\mu$ eines Punktes zufällig ausgewählt wird und somit die vorgegebene Unordnung des Systems. Rot (grün) dargestellt sind leitfähige (isolierende) Bereiche. Als Resultat dieser Simulationen ergibt sich eine

\footnotetext{
${ }^{30} \mathrm{~W}$ definiert das Intervall $[-W ; W]$, aus dem zufällig chemische Potentiale $\mu$ ausgewählt werden.
} 
Clusterbildung von antiferromagnetischen und ferromagnetischen Bereichen, die aufgrund des variierenden Kopplungsparameters zwar unterschiedlich groß ausfallen, aber in allen drei Bildern eine vergleichbare Struktur aufweisen. Diese Ergebnisse entsprechen strukturell recht gut den in Kapitel 5 durchgeführten Untersuchungen. In (b) ist ein Ausschnitt der Messung aus Abbildung 5.2 (b) ${ }^{31}$ mit einer Größe von $118 \mathrm{~nm} \times 118 \mathrm{~nm}$ dargestellt. Diese Darstellung weist starke strukturelle Ähnlichkeiten mit den Simulationen auf und ist aufgrund des Metall - Isolator Verhältnisses zwischen den Darstellungen (c1) und (c2) anzusiedeln.

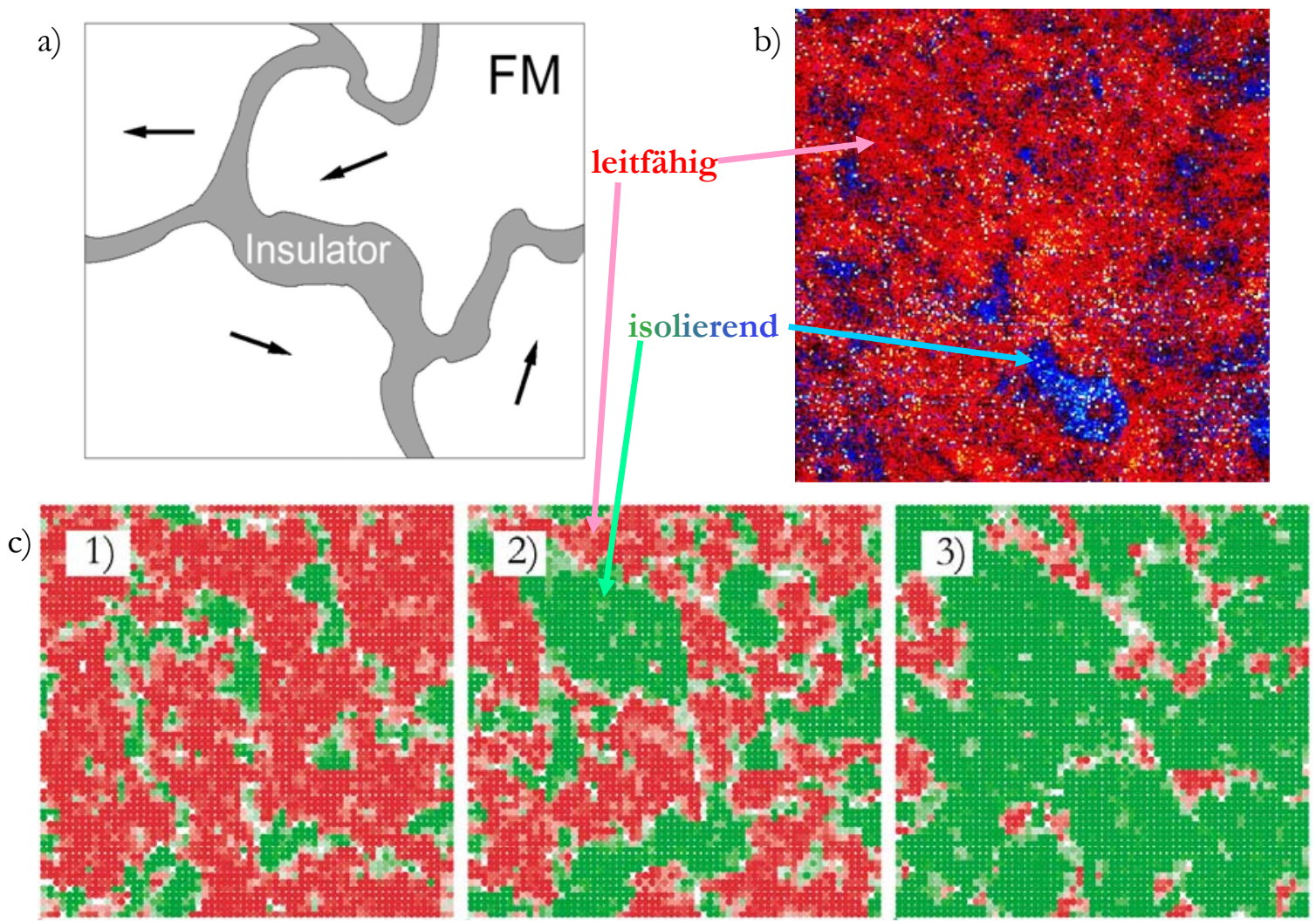

Abbildung 6.7: In (a) ist ein Modell für eine mögliche Domänenstruktur skizziert. Ein ungleichmäßig strukturierter, isolierender Bereich trennt metallische Bereiche voneinander. Die Pfeile symbolisieren die magnetische Ausrichtung der Domänenstruktur. In Abbildung (b) ist die kombinierte Leitwert - und Isolationskarte mit der Kantenlänge von $118 \mathrm{~nm}$ einer Messung LCMO auf MgO aus Abbildung 5.2 (b) dargestellt (rot: leitfähig, blau: isolierend). (c1-3) zeigt die Ergebnisse von MC-Simulationen für variierende Kopplungsparameter. Rot (grün) dargestellt sind leitfähige (isolierende) Bereiche [7].

In einer weiterführenden Arbeit hat Burgy [91; 92] den Einfluss der Präparationsbedingungen auf die Domänenstruktur des elektronischen Gefüges untersucht und in die bisher durchgeführten Monte - Carlo - Simulationen implementiert. Entsprechend einer Oberfläche, die ihre

\footnotetext{
$3150 \mathrm{~nm}$ LCMO auf MgO, gemessen bei einer Temperatur von $78 \mathrm{~K}$
} 
Oberflächenenergie durch Glättung verringern will, können auch Domänenstrukturen einem glättenden Mechanismus unterworfen werden. Wie bisher gezeigt wurde, sind die zu erwartenden leitfähigen und isolierenden Cluster von einer Größe, die unter zu Hilfenahme eines Standard Perkolationsmodell (SP) ${ }^{32}$ nicht erreicht werden können. Abbildung 6.8 (a) zeigt eine Berechnung bestehend aus $128 \times 128$ Gitterpunkten bei tiefer Temperatur ohne die Einflüsse von Potentialen (z.B. dem chemischen Potential $\mu$ ) oder Kopplungsparametern. Ersichtlich ist ein Verhalten, dass einem „Rauschen“ gleicht - Leitfähige Pixel (weiß) und isolierende (schwarz) liegen ungeordnet nebeneinander vor, wodurch keine zusammenhängenden Strukturen vorhanden sind. Werden nun die oben genannten Einflüsse in der Simulation beachtet, ergibt sich eine Struktur, die in vergleichbarer Form bisher schon häufiger vorgestellt wurde ${ }^{33}$.
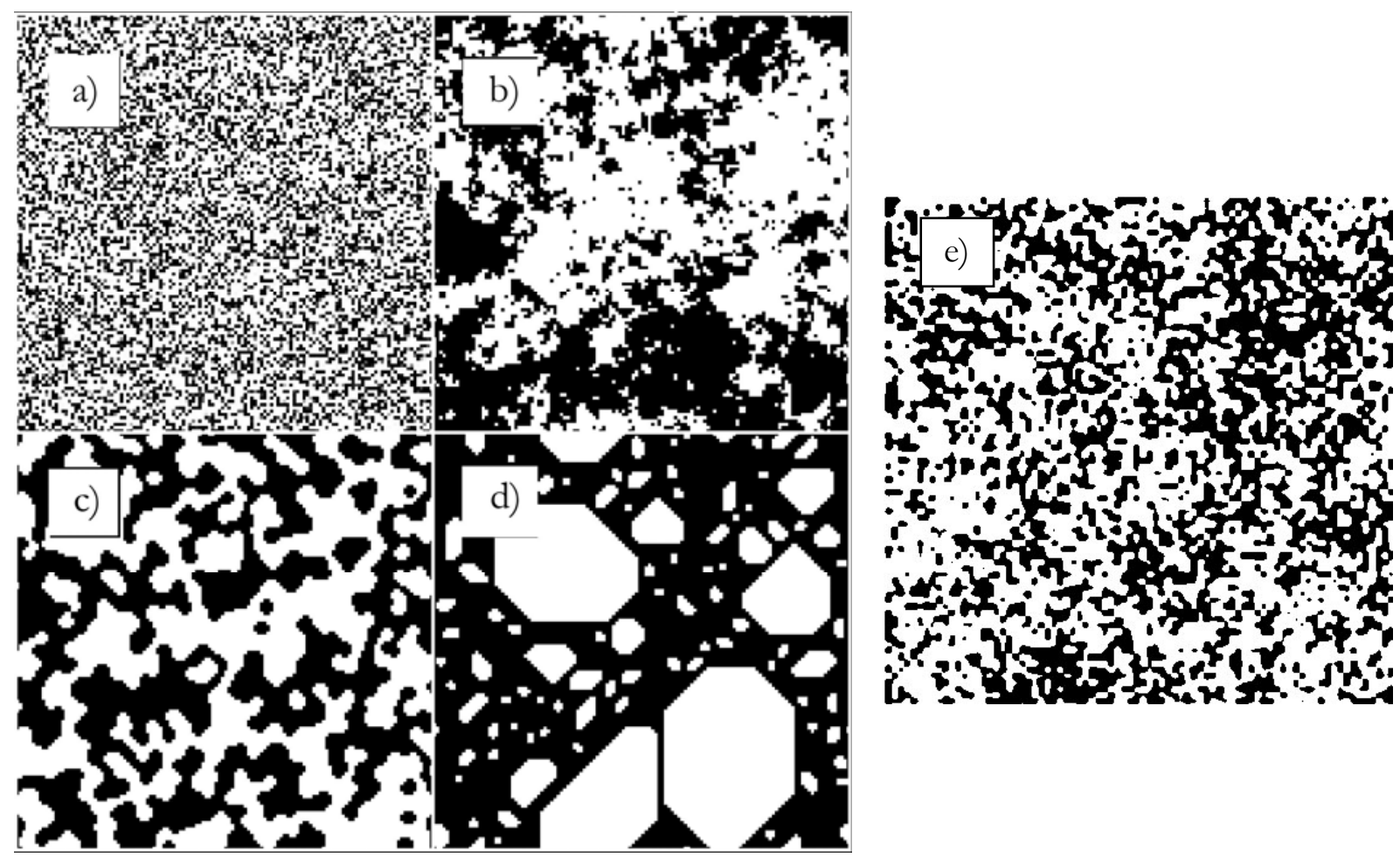

Abbildung 6.8: Unterschiedliche Clusterbildungen durch verschiedene Parameter bei der MCSimulation sind in (a) bis (d) dargestellt. In (e) befindet sich eine Leitwertkarte aus Kapitel 5.4 gemessen an einem LSMO - Film auf MgO bei 150 K. Der Scanbereich beträgt $500 \mathrm{~nm} \times 500 \mathrm{~nm}$. Weiße Bereiche signalisieren leitfähiges Verhalten, schwarze Regionen sind isolierend [91].

Eine recht ungeordnete Domänenstruktur ist als Ergebnis in Abbildung (b) dargestellt. Tendenziell handelt es sich um ein erwartetes Resultat, einzig die rein zufällige und stark filigrane

\footnotetext{
32 Es beschreibt eine rein statistische Verteilung.

33 z.B. auf den Seiten 20, 23 und 80
} 
Bereichsentwicklung entspricht nicht den Erwartungen, die aufgrund der bisherigen Ergebnisse an Manganaten geprägt wurden. Erst die Addition von zusätzlichen Mechanismen zeigt eine Glättung der Domänenstrukturen, entsprechend der Abbildungen (c) und (d). Hierzu wurde beachtet, dass die elektronischen Eigenschaften einer beliebigen Zelle durch ihre Nachbarschaft beeinflusst werden können. Dies entspricht dem langreichweitigen elektronischen Charakter, hervorgerufen von elastischen Gitterverzerrungen bei Manganaten [93]. Befindet sich in der Nähe einer zu beobachtenden Zelle eine ausreichend hohe Anzahl von Nachbarn mit den gleichen elektronischen Eigenschaften, so ändert sich nichts. Falls dies aber nicht der Fall ist und sich in der Nachbarschaft eine gewisse Anzahl von Zellen mit konkurrierender Phase befindet, kann die Ausgangszelle ihre Eigenschaften zugunsten der anderen Phase wechseln. Dementsprechend sind in den Abbildungen (c) und (d) zwei unterschiedliche Regeln angewandt worden, um Perkolation und Clusterbildung durch Koagulation zu simulieren. Perkolatives Verhalten lässt sich vereinfacht durch das Modell MGE5 darstellen [94]. Dieses Modell basiert auf der Mooreschen Nachbarschaft ${ }^{34}$ und beschreibt die Reaktion einer Zentralzelle auf ihre Umgebung. Sobald mindestens fünf von neun Zellen metallisches Verhalten aufweisen, wird bzw. bleibt die Zentralzelle metallisch. Dieses Modell ist von Burgy auf die Verteilung aus Abbildung (a) angewandt worden und als Ergebnis in Abbildung (c) dargestellt. Deutlich zeichnet sich ein vergleichbares Bild mit den Ergebnissen aus dieser Arbeit ab. Zum Vergleich ist in Abbildung (e) die Leitfähigkeitsverteilung mit angewandtem Schwellwert für LSMO auf MgO (Kapitel 5.4) bei $150 \mathrm{~K}$ dargestellt. Beide Verteilungen besitzen den gleichen strukturellen Charakter, wobei die Simulation entsprechend einer Vergrößerung aus der Messung wirkt (vgl. Abbildung 6.8 (c) und (e)). Wird indes die MGE4 ${ }^{35}$ Regel auf die Ursprungsverteilung angewandt, ergibt sich Nukleation (Abbildung (d)). Hierbei wachsen größere Cluster auf Kosten der kleineren - mit perkolativem Verhalten ist somit erst bei sehr hohen Konzentrationen der metallischen Phase zu rechnen.

Es ist jedoch zu beachten, dass bisher nur zweidimensionale Systeme bzw. Oberflächen betrachtet wurden. Bei Transportphänomenen innerhalb einer Bulkprobe handelt es sich jedoch um dreidimensionale Vorgänge und somit um abweichende Voraussetzungen für perkolatives Verhalten. Die unterschiedliche Anzahl an Dimensionen hat Einfluss auf den prozentualen Anteil der leitfähigen Phase, bei der ein System gerade noch perkoliert und ein Netzwerk ausbilden kann. Dieser Wert wird als kritischer Parameter $p_{c}$ bezeichnet und stellt das minimale Verhältnis zwischen leitfähigen und isolierenden Bereichen oder Volumina dar. Wird dieser Wert

\footnotetext{
34 Die Bereiche in der Umgebung einer Zelle, die dieser "bekannt" oder "für sie sichtbar" sind, bilden ihre 'Nachbarschaft'. Bei der Mooreschen Nachbarschaft handelt es sich um 8 zu einem Quadrat um die Zentralzelle angeordnete Bereiche (weiterführende Informationen sind unter [94] zu finden)

35 entsprechend MGE5 werden hier nur vier Nachbarzellen betrachtet
} 
unterschritten wird der Stromfluss erheblich beeinträchtigt bzw. unterbrochen. In einem zweidimensionalen Netzwerk, bestehend aus statistisch verteilten, quadratischen Zellen, bestimmt sich $p_{c}$ zu 0.5 - es müssen also mindestens $p>50 \%$ einer Phase vorliegen, damit ein Netzwerk ausgebildet werden kann. Für ein dreidimensionales, einfach kubisches Netzwerk liegt $p_{c}$ etwas niedriger bei 0.31 [95].

Um einen Vergleich der zweidimensionalen Domänenverhältnisse aus den STS - Messungen mit den dreidimensionalen bulk - Eigenschaften schließen zu können, werden im Folgenden die Magnetisierungsmessungen am Einkristall (Kap. 5.3) und an dem dünnen LSMO - Film aus Kapitel 5.4 zur Diskussion herangezogen. Abbildung 6.9 zeigt grün dargestellt die theoretische Magnetisierung entsprechend der Molekularfeld - Näherung [96] und die am LSMO - Einkristall durchgeführte Magnetisierungsmessung (schwarze Rechtecke). Beide Kurvenverläufe sind auf ihren Wert bei tiefster Temperatur normiert worden. Hierbei muss jedoch beachtet werden, dass bei dieser Temperatur der Volumenanteil der metallischen Phase innerhalb des ManganatEinkristalls nicht zwangsläufig den Wert von $100 \%$ besitzen muss. Dementsprechend ist ein zu tieferen Magnetisierungswerten versetzter Verlauf der experimentell bestimmten Kurve im Vergleich zu den eingezeichneten, theoretischen Werten denkbar.

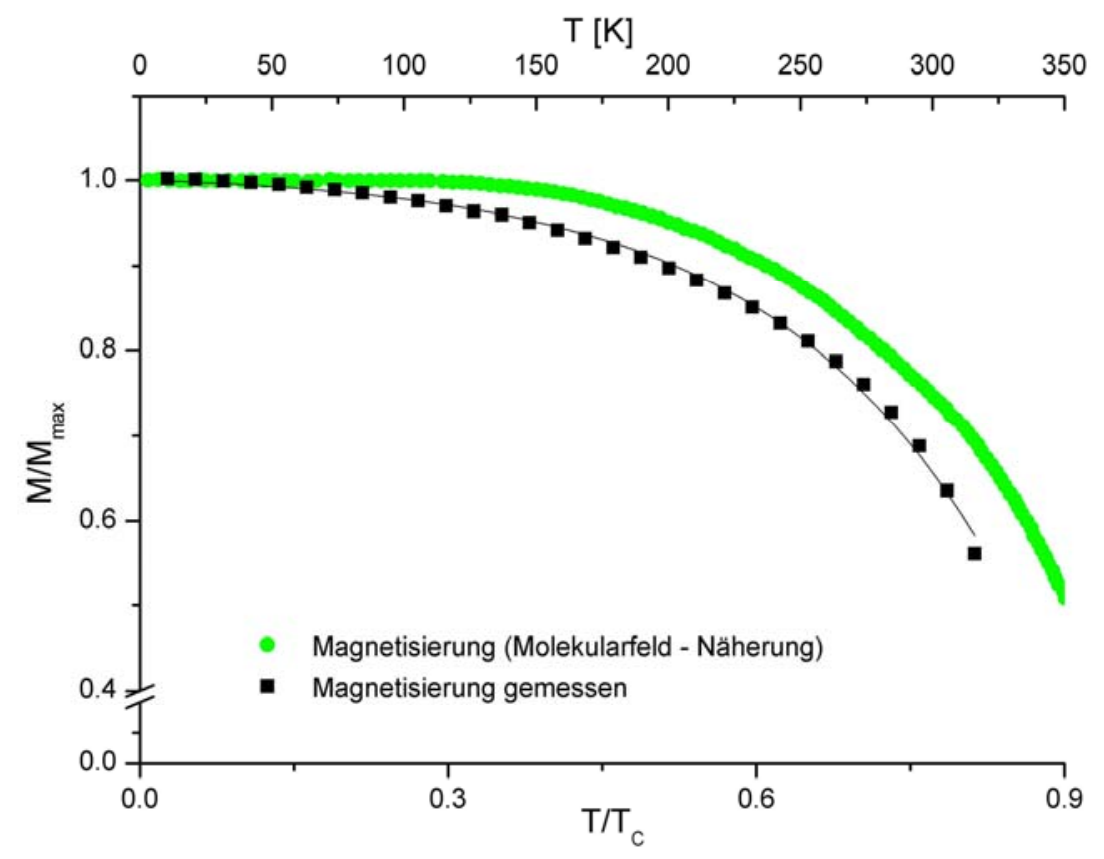

Abbildung 6.9: Vergleich der grün dargestellten, theoretischen Magnetisierung entsprechend der Molekularfeld - Näherung [96] mit der normierten Messung an dem LSMO - Einkristall aus Kapitel 5.3 (schwarze Rechtecke)

Sofern innerhalb des Einkristalls nur metallisches Verhalten und keine wachsenden isolierenden Domänen anzutreffen wären, sollten beide Kurven deckungsgleich verlaufen. Die beiden 
Graphen in Abbildung 6.9 zeigen aber, dass dies nicht der Fall ist: Die Magnetisierungsmessung verläuft bei tiefen Temperaturen sichtbar steiler und zeigt zu höheren Temperaturen hin eine niedrigere Magnetisierbarkeit als theoretisch vorhergesagt ist. Dieses Ergebnis weist darauf hin, dass zusätzlich zur temperaturabhängigen Abnahme der Magnetisierbarkeit ein weiterer Mechanismus existiert, der auf eine Volumenabhängigkeit hindeutet.

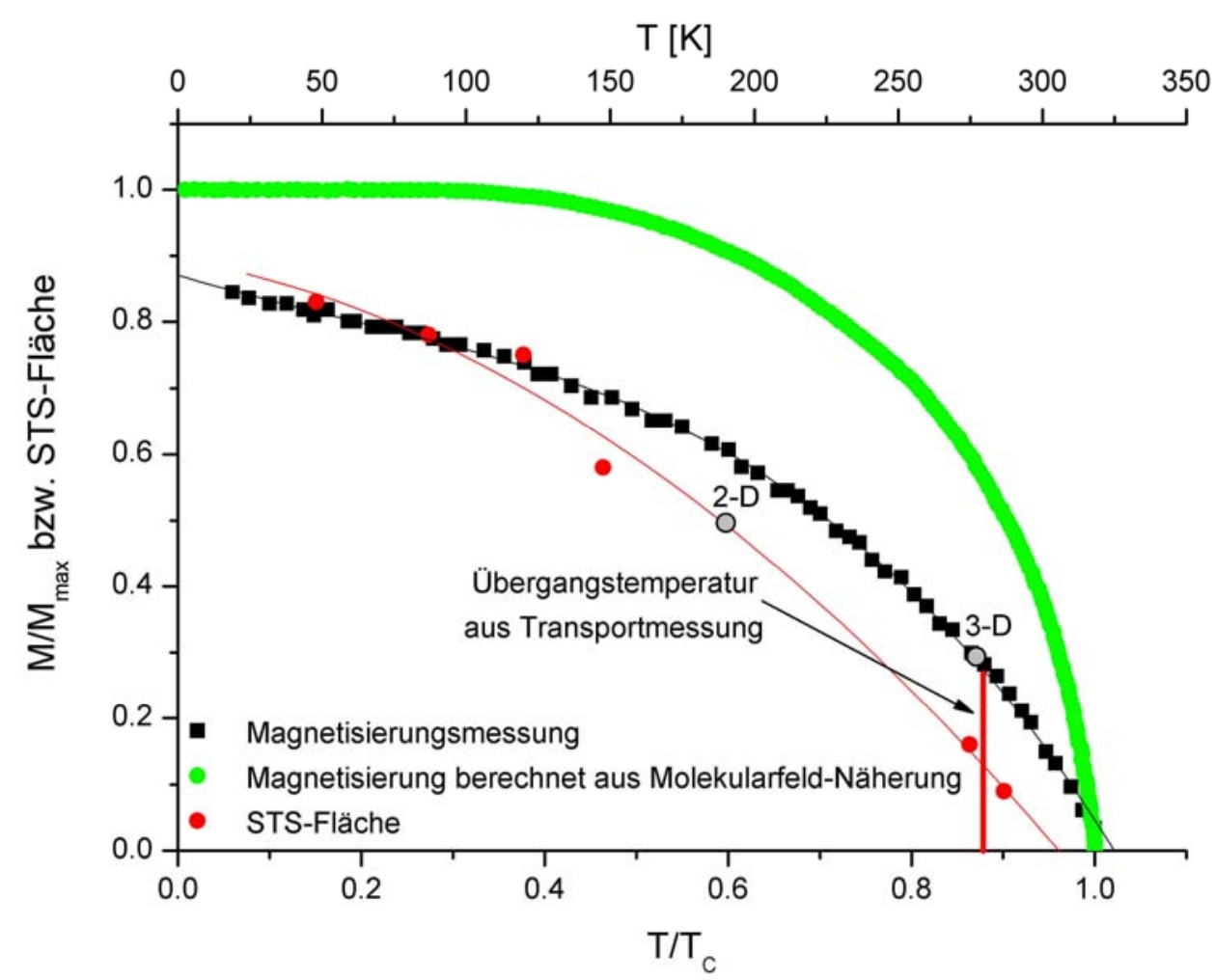

Abbildung 6.10: Grün dargestellt ist die theoretische Magnetisierung nach der Molekularfeld - Näherung (entsprechend Abbildung 6.9). Die schwarzen Rechtecke stellen die Magnetisierungsmessung an dem dünnen LSMO-Film aus Kapitel 5.4 dar. Zur Normierung dieser dient der prozentuale Anteil der als metallisch leitfähig definierten Domänen aus den STS - Messungen (rote Kreise) bei tiefen Temperaturen. Die grauen Kreise deuten die kritischen Parameter $p_{c}$ für zwei - und drei - dimensionale Systeme an. Die rot eingezeichnete Senkrechte gibt die Übergangstemperatur aus den Transportmessungen wieder.

Dementsprechend werden die temperaturabhängigen STS - Flächenanteile der metallischen Domänen bei dem dünnen LSMO-Film aus Kapitel 5.4 zur Normierung der Magnetisierungsmessung herangezogen (Abbildung 6.10). Der theoretische Kurvenverlauf (grün) ist bei tiefen Temperaturen konstant und zeigt bis ca. $0.4 T_{C}$ keine signifikante Änderung. Dies beschreibt, dass das magnetische Moment und das Volumen der Probe in diesem Bereich als konstant anzusehen ist. Demgegenüber weist die Magnetisierungsmessung (schwarze Rechtecke) innerhalb dieses Bereiches eine Abnahme auf. Da sich das magnetische Moment entsprechend 
der theoretischen, grün dargestellten Kurve nicht ändert, ist diese Abnahme nur auf die Verminderung des Volumenanteils zurückzuführen. Dieses Verhalten entspricht der Reduzierung des prozentualen Anteils der metallischen STS - Flächen und ist auf diese (rote Kreise) normiert worden. Bei tiefen Temperaturen liegt eine sehr gute Übereinstimmung zwischen den Flächenanteilen und der gemessenen Magnetisierung vor und zeigt über den gesamten Temperaturbereich eine der Magnetisierungsmessung entsprechende Form (rote Kurve). Einzig zu höheren Temperaturen ist eine Abweichung zu geringeren Werten hin festzustellen. Für diese Unterschiede gibt es mehrere Ansätze die Erklärungspotential liefern können:

Dowben und Skomski [97; 98] postulieren eine sehr dünne, isolierende Schicht, die sich direkt an der Oberfläche der Probe befindet. Als Grund hierfür können die nicht abgeschlossenen, chemischen Bindungen bzw. die daraus resultierenden strukturellen Veränderungen in Betracht gezogen werden. Die Existenz dieser Schicht würde die vorhandene Problematik bei STM Messungen und die fehlende atomare Auflösung an diesen Materialien erklären. Giessen [99] diskutiert in diesem Zusammenhang die Störung der $\mathrm{Mn}-\mathrm{O}-\mathrm{Mn}$ Bindungen an der Oberfläche und in den Korngrenzen, woraus eine Einflussnahme auf den Doppelaustausch resultiert. Somit ist an diesen Stellen mit einem im Vergleich zur Volumenprobe erhöhten prozentualen Flächenanteil der isolierenden Bereichen zu rechnen. Dieses Verhalten würde den im Vergleich zu der Magnetisierungsmessung zu tief verlaufenden metallischen STS - Anteil erklären.

Generell haben alle in dieser Arbeit durchgeführten und dargestellten Messungen gezeigt, dass keine scharfe Domänengrenzfläche zwischen zwei unterschiedlich leitfähigen Bereichen besteht. In Abbildung 6.11 (a) ist zur Veranschaulichung die Leitwert - bzw. Gapbreitenverteilung aus Kapitel 5.2 (Seite 48) nochmals aufgetragen. Die Darstellung in (b) zeigt die gleiche Verteilung wie in (a), jedoch wurden dem Ausgangsbild die Farbinformationen entzogen. Die resultierende Helligkeitsverteilung zeigt deutlich Unterschiede, die sich in der Nähe der Domänengrenzen aus Abbildung (a) befinden. Eine Analyse der Randbereiche ergibt für diese eine durchschnittliche Breite von ca. $3 \mathrm{~nm}$. Das Histogramm dargestellt in (c) zeigt deutlich die Vorherrschaft mittlerer Graustufen (2), die den metallischen und isolierenden Domänen entstammen. Links von diesem zentralen Maximum befindet sich eine breite, auslaufende Schulter (1), die aus den dunkleren Farbwerten resultiert. Diese dunklen Bereiche aus (b), die sich in der Nähe der Domänengrenzen befinden, könnten durch Spannungseinflüsse der strukturell veränderten isolierenden Phase auf die metallischen Domänen herrühren. Als Hintergrund hierfür kann angebracht werden, dass der Doppelaustausch eine starke Winkelabhängigkeit besitzt und somit in der Nähe einer Domänengrenze durch strukturelle Variationen der isolierenden Domänen beeinflusst werden 
kann. Entsprechend der oben bestimmten Bereichsbreiten würde sich diese Einflussnahme auf ca. $3 \mathrm{~nm}$ belaufen. Dementsprechend existiert die offene Frage, ob die Spins der Elektronen innerhalb dieser teilweise isolierenden Bereiche nicht doch noch ausgerichtet sind und ein magnetisches Moment einbringen. Durchgeführte NMR $^{36}$ - Untersuchungen von Bibes [100] weisen auf eine entsprechende Koexistenz von ferromagnetisch leitfähigen, ferromagnetischen isolierenden und antiferromagnetisch isolierenden Phasen innerhalb der Volumenprobe hin. Dies würde einen etwas erhöhten Verlauf der Magnetisierungskurve im Vergleich zu dem bestimmten Metall - Isolator Verhältnis (STS - Fläche aus Abbildung 6.10) erklären.

a)

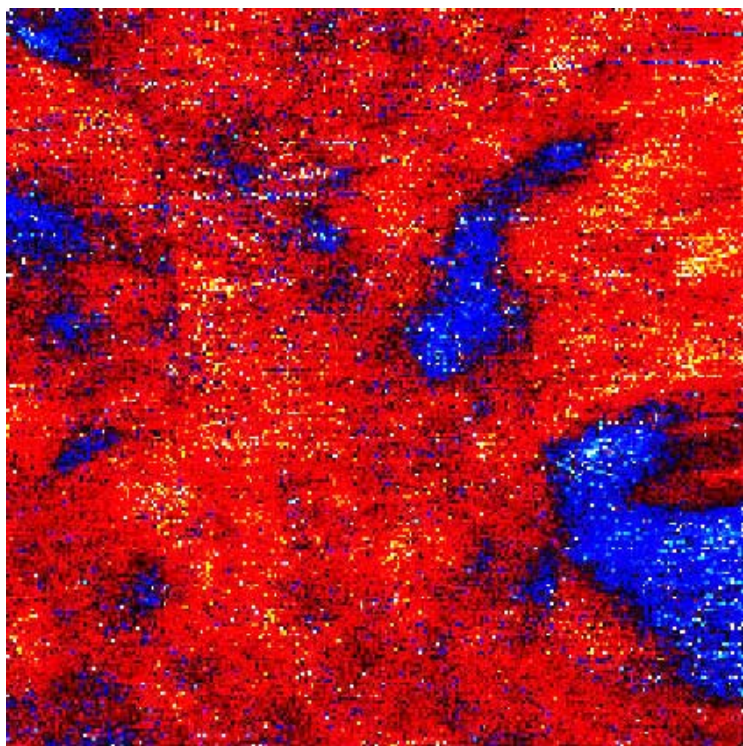

b)

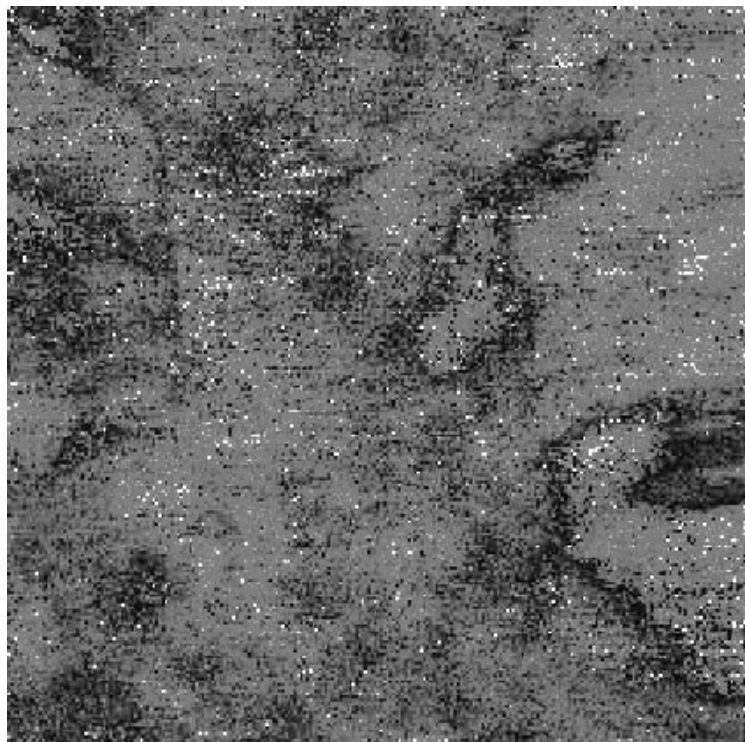

c)

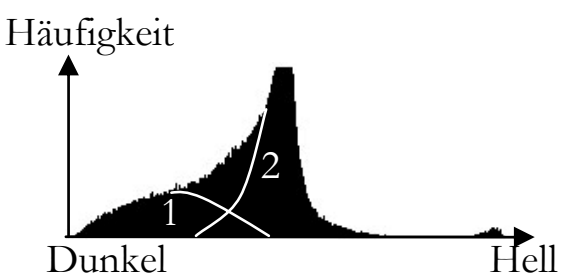

Abbildung 6.11: a) Leitwert- und Gapverteilungskarte von LCMO $(65 \mathrm{~nm} \times 65 \mathrm{~nm}$ ) aus Kapitel 5.2 (Seite 48)

b) entsprechend (a), nur auf Helligkeitsunterschiede reduziert

c) Histogramm entsprechend Abbildung (b), (1) dunkel dargestellte Bereiche; (2) mittlere Grautönung aus metallischen und isolierenden Bereichen.

Zusätzlich sind in dem Graphen aus Abbildung 6.10 die oben genannten Werte für zwei- und dreidimensionale Perkolation als graue Kreise eingetragen. Handelt es sich um eine $2-$ dimensionale Struktur entsprechend den STS - Flächenanteilen, liegt die Perkolationsschwelle bei $50 \%$. Ist wie bei der Magnetisierungsmessung eine 3-dimensionale bulk-Messung

\footnotetext{
${ }^{36}$ Nuclear Magnetic Resonance
} 
vorgenommen worden, bestimmt sich die Schwelle zu $31 \%$. Für diesen zweiten Fall zeigt sich eine sehr gute Übereinstimmung mit der experimentell bestimmten Übergangstemperatur aus der Transportmessung (rote Linie). Dieses Ergebnis lässt den Rückschluss zu, dass die durchgeführte Normierung der Magnetisierungskurve über die STS - Flächenanteile gerechtfertigt ist. Für das 2 - dimensionale System ist die Perkolationsschwelle und somit eine Widerstandsänderung bei $0.6 T_{C}(\sim 200 \mathrm{~K}) \mathrm{zu}$ erwarten. Die Resultate der Netzwerksimulationen dargestellt in Abbildung 5.13 auf Seite 58 zeigen hierzu ein konformes Ergebnis. Unterhalb der Temperatur von $200 \mathrm{~K}$ verlaufen die simulierten Widerstände bei niedrigen Temperaturen, oberhalb ist ein Anstieg des Widerstandes festzustellen. Dieser ist aufgrund der gegebenen Widerstände nicht so scharf wie erwartet. Sofern nur zwei Typen von Widerständen vorliegen würden (niederohmiger Widerstand: leitfähige Bereiche; hoher Widerstand: isolierende Domänen), würde ein solcher sprunghafter Verlauf vorliegen. Da aber isolierenden Bereichen teilweise eine Restleitfähigkeit zugesprochen wird (vgl. Histogramm dargestellt in Abbildung 5.12 auf Seite 56) ist mit nur einer dem Graphen entsprechenden, breitverteilten Zunahme zu rechnen. 


\section{Zusammenfassung}

In dieser Arbeit wurden Manganatschichten und Einkristalle vom Typ $\mathrm{La}_{x} \mathrm{Ca}_{1-\mathrm{x}} \mathrm{MnO}_{3}$ (LCMO) und $\mathrm{La}_{\mathrm{x}} \mathrm{Sr}_{\mathrm{x}-1} \mathrm{MnO}_{3}$ (LSMO) mittels Rastersondenmikroskopie (STM) und - Spektroskopie (STS) auf ihre elektronischen Eigenschaften an der Oberfläche untersucht. Eine vorliegende Phasenseparation bildet die Grundlage, um die ungewöhnlichen Widerstandsänderungen am Metall - Isolator - Übergang über ein Perkolationsmodell von isolierenden und metallischen Domänen zu verstehen. Aufgrund der unterschiedlich verlaufenden Kennlinien ist es möglich, die vorliegenden Phasen voneinander zu separieren und ortsaufgelöste Leitfähigkeitskarten zu erstellen. Eine Kombination aus diesen Karten und den zugehörigen topographischen Informationen zeigt, dass nur eine schwache Korrelation zwischen den elektronischen Domänen und der Kornstruktur vorliegt - einzelne Körner weisen elektronische Inhomogenitäten auf.

Es wurde gezeigt, dass die Leitfähigkeit sich als Funktion der Temperatur im Wesentlichen aufgrund der intrinsischen Inhomogenitäten der Probe ändert und dass zusammenhängende Bereiche verschiedener Größe und Leitfähigkeit existieren. Die durchgeführten Messungen an dünnen Filmen und Einkristallen erstreckten sich innerhalb eines Temperaturbereiches von $37 \mathrm{~K}$ bis Raumtemperatur, wobei die isolierenden Domänen mit zunehmender Temperatur wachsen und letztlich die Strompfade innerhalb der metallisch leitfähigen Phase bei der Übergangstemperatur durchschneiden.

Um eine Trennung von metallisch leitfähigen Bereichen und isolierenden Domänen durchführen zu können, wurde ein Schwellwert eingeführt. Er definiert sich aus der Anpassung der leitfähigen Bereiche aus den STS-Messungen (2 dimensional) an die an den Schichten gemessenen Magnetisierungskurven (3 dimensional). Somit ist sichergestellt, dass die oberflächensensitiven STS - Messungen und die Volumenmagnetisierung bei tiefen Temperaturen konsistent sind. Diese Vorgehensweise wird von Leitfähigkeitsverteilungen (entsprechend Abbildung 5.12) gestützt, die eine deutliche Trennung von leitfähigen und isolierenden Bereichen aufzeigen und eine vergleichbare Definition des Schwellwerts ermöglichen.

Um den gemessenen Transportwiderstand direkt mit dem aus den STS - Kennlinien bestimmten Tunnelwiderstand vergleichen zu können, wurde eine quantitative Analyse durchgeführt. Diese analytische Methode ermöglicht es erstmals, alle gemessenen Tunnelleitfähigkeiten innerhalb eines Widerstandsnetzwerkmodells zur Bestimmung dessen Gesamtwiderstandes zu nutzen. Damit unterscheidet sich diese neue Methode fundamental von den bisherigen Modellen [9], weil es sich um eine rein numerisches Verfahren handelt, das lediglich die Kirchhoffschen Regeln 
verwendet. Die daraus resultierenden Ergebnisse konnten zur Modellierung der Transportwiderstände genutzt werden und zeigen ein den Transportmessungen entsprechendes Übergangsverhalten, wobei auch hier ein stark verbreiterter Übergang zu erkennen ist.

Zusätzlich erlaubt das Widerstandnetzwerkmodell die Darstellung von Stromkarten und der ortsaufgelösten Strompfaddichte. Nahe unterhalb der Übergangstemperatur lassen sich dementsprechend Stromverlaufskarten generieren, die eindeutig das perkolative Verhalten dieser Materialklasse verdeutlichen, indem sie nur noch wenige, jedoch stark ausgeprägte Strompfade zeigen, die bei weiterer Temperaturerhöhung unterbrochen werden. Als weiteres Ergebnis der Simulation lassen sich die Stromflussentwicklungen über einen definierten Probenbereich sichtbar machen.

Offen bleibt jedoch die Frage, ob die elektronische Domänenstruktur mit der magnetischen identisch ist, oder ob isolierende Bereiche existieren, die ein magnetisches Moment zeigen. MFM - Messungen besitzen eine zu niedrige Ortsauflösung, wodurch ein direkter Vergleich mit STS - Analysen im Ansatz scheitert. Hier könnten spinpolarisierte STS Messungen die Auflösung verbessern [101]. Dieses Verfahren bietet die derzeit höchste erreichbare Auflösung für magnetische Strukturen und kann Hinweise entsprechend der oben angesprochenen Fragestellung liefern. 


\section{Anhang}

\subsection{Sauerstoffdegradation}

Die dieser Arbeit vorangegangenen STM - und STS - Messungen an Manganaten erwiesen sich anfangs als erfolglos, da frisch präparierte LCMO - und LSMO - Filme nur wenige Tage im UHV bei Raumtemperatur untersucht werden konnten. Es zeigte sich vielmehr, dass die STM Spitze nach kurzer Zeit die Probenoberfläche während der Messung strukturiert. Zur Klärung dieses Phänomens ist als erstes untersucht worden, ob und in welchem Maße Nahfeldverdampfung dafür zuständig sein kann. An einem gesputterten, $90 \mathrm{~nm}$ dicken LCMO Film, der sich seit ca. 5 Tagen im UHV befand, sind hierfür insgesamt fünf verschiedene Messungen mit einer Tunnelspitze durchgeführt worden:

vier mit unterschiedlich eingestellten Spannungen $U_{T}$ bei festem Tunnelstrom $I_{T}$ eine letzte als Übersichtsmessung.

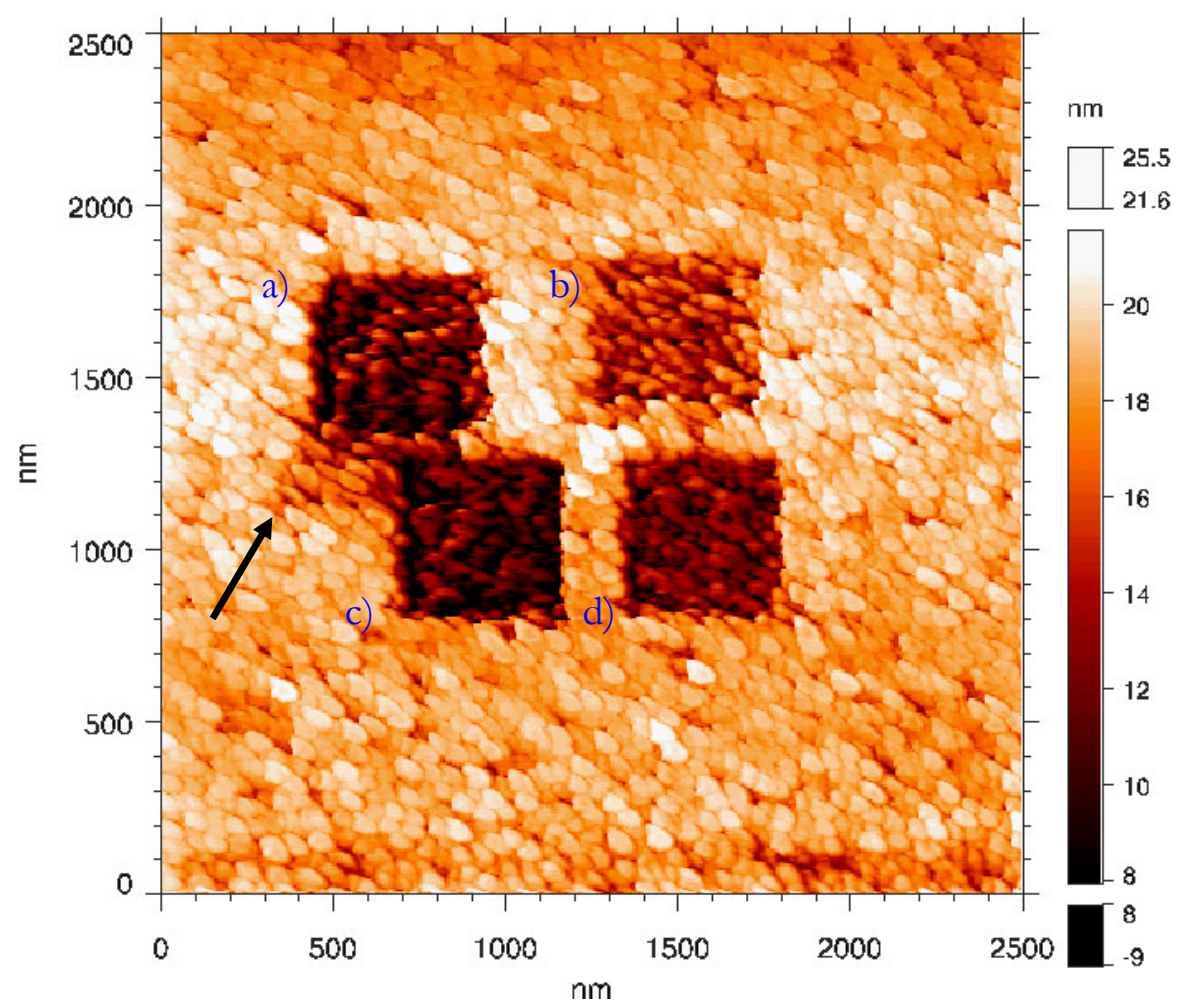

Abbildung 8.1: STM - Aufnahme einer strukturierten LCMO - Probe. Die Bereiche (a) bis (d) wurden mit unterschiedlichen Tunnelspannungen gemessen. 
Jeder Testmessungsbereich aus Abbildung 8.1 (a) bis (d) besitzt eine Dimension von $(500 \mathrm{~nm})^{2}$ und ist insgesamt dreimal abgerastert worden. Um immer einen festen Abstand zwischen Spitze und Probe zu gewährleisten, ist für alle Aufnahmen ein Tunnelstrom $I_{T}=0,1 \mathrm{nA}$ gewählt worden. Die Tunnelspannungen $U_{T}$ unterscheiden sich für die Messungen wie folgt:

\begin{tabular}{|c|c|c|}
\hline Messung aus Abbildung 8.1 & Tunnelspannung $\boldsymbol{U}_{\boldsymbol{T}}$ & Mittlere Grabentiefe \\
\hline a) & $0,01 \mathrm{~V}$ & $15 \mathrm{~nm}$ \\
\hline b) & $0,1 \mathrm{~V}$ & $11 \mathrm{~nm}$ \\
\hline c) & $-0,01 \mathrm{~V}$ & $13 \mathrm{~nm}$ \\
\hline d) & $-0,1 \mathrm{~V}$ & $12 \mathrm{~nm}$ \\
\hline
\end{tabular}

Für den ersten Durchlauf in Messung (a) ist eine anfänglich starke Drift festzustellen (siehe Pfeil in Abbildung 8.1), die bei den zwei Folgenden jedoch kaum noch zu erkennen ist. Sollte die Nahfeldverdampfung für den Strukturierungseffekt zuständig sein, müssten Unterschiede in der Grabentiefe erkennbar sein. Messbereiche mit niedriger Spannung müssten im Vergleich zu denen mit hohen Werten niedrigere Materialabtragungen vorweisen. Eine Bertachtung der Grabentiefe zeigt aber bei allen vier Zellen einen mittleren Wert von 12,75 $\mathrm{nm}^{37}$ und steht in keinem Bezug zu den angelegten Tunnelspannungen. Eine Form der Feldverdampfung kann somit ausgeschlossen werden.

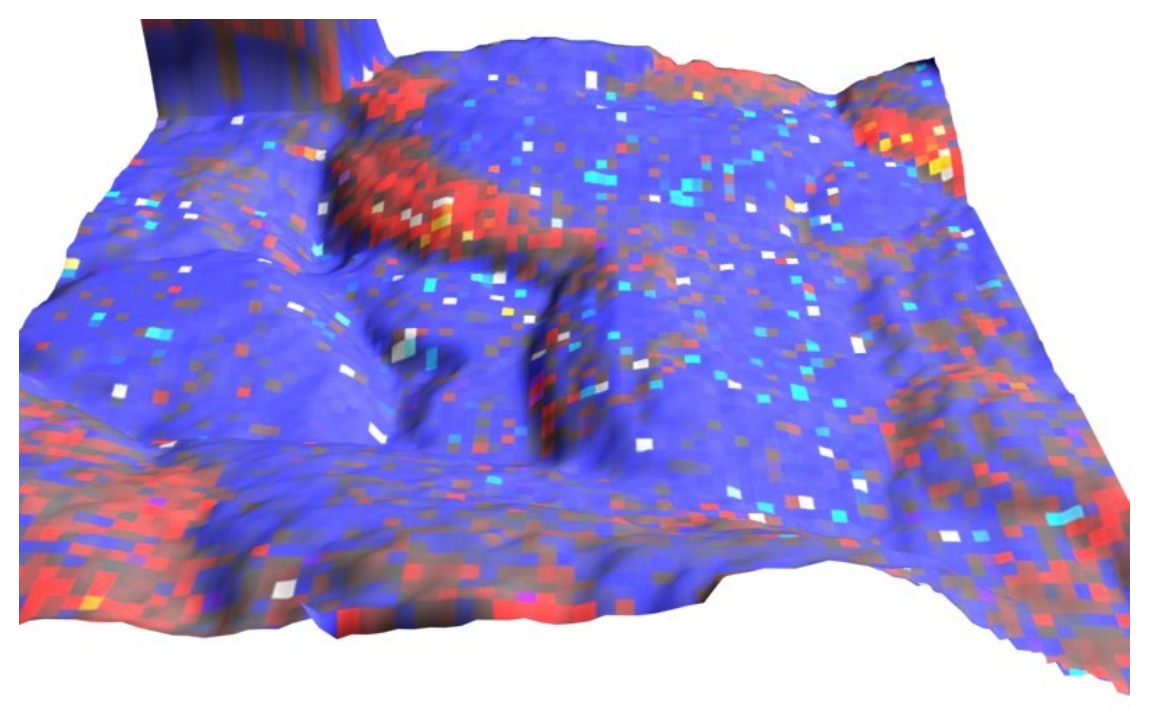

Abbildung 8.2: Kombination aus Topographie, Leitwert- und Gapkarte. Das Bild zeigt einen Ausschnitt von $75 \mathrm{~nm} \times 75 \mathrm{~nm}$. Leitfähige (isolierende) Bereiche sind rot (blau) dargestellt.

\footnotetext{
${ }^{37}$ Die leichten Höhenunterschiede der Messbereiche sind auf die Verkippung bei dem durchgeführten Linefit zurückzuführen.
} 
Weiteres Erklärungspotential hinsichtlich der Materialabtragung besitzt die Sauerstoffdegradation im Vakuum. Bleibt ein Manganatfilm für ca. eine Woche im UHV, so ist es nicht mehr möglich, an ihm STM-Messungen vorzunehmen. Dieser Effekt wird der Sauerstoffdegradation entstehend durch temperaturabhängige Diffusion im UHV zugesprochen. Sinkt der Sauerstoffgehalt der Probe nahe der Oberfläche, wird der Anteil an $\mathrm{Mn}^{4+}$ - Ionen reduziert und somit die Ladungsträgerdichte verringert [102]. Die Probenoberfläche wird so hochohmig, dass sie mit dem STM nicht mehr messbar ist.

Ein LSMO - Film auf MgO ist, nachdem er sich für ca. eine Woche bei Raumtemperatur im Vakuum befand, mit rastersondenmikroskopischen Methoden nicht mehr zugänglich. Daraufhin findet eine Aufladung mit Sauerstoff durch Auslagerung der Probe bei $400^{\circ} \mathrm{C}$ für drei Stunden an Luft statt. Eine anschließende Messung im UHV bei Raumtemperatur zeigt, dass es nun möglich ist, diese Probe zu messen. Abbildung 8.2 zeigt die Kombination des dreidimensionalen STM Bildes mit der Leitwert- und Gapverteilungskarte. Deutlich ist die Häufung von rot dargestellten, leitfähigen Bereichen nahe den Korngrenzen zu erkennen. Die Kornoberflächen zeigen eher halbleitenden Charakter (blau dargestellt). Dementsprechend lässt dies die Schlussfolgerung zu, dass der Sauerstoff leichter durch Korngrenzen in den Film eindringt als direkt über die Oberfläche. 


\subsection{Ergebnisse an Vanadiumoxid}

Einige Oxide des Vanadiums zeigen einen temperaturabhängigen Übergang zwischen einer metallischen und einer isolierenden Phase. Dieser Metall - Isolatorübergang beruht jedoch im Vergleich zu Manganaten auf einem anderen Mechanismus. Grundlage hierfür ist das MottHubbard - Modell [103], das seit dreißig Jahren als Ausgangspunkt zur Diskussion genutzt wird. Es beschreibt eine Lokalisierung der Leitungs - Elektronen aufgrund der starken CoulombWechselwirkung. Bei vorliegender isolierender Phase ist das 3d-Leitungsband in zwei Teilbänder mit einer Breite $W$ und einem Abstand $U$ aufgespalten. Existiert keine Überlappung, ist also $U>W / 2$, existieren keine freien Zustände nahe der Fermi-Energie und der Ladungstransport kann nur über thermisch angeregte Elektron - Loch - Paare als Ladungsträger erfolgen.

$\mathrm{V}_{2} \mathrm{O}_{3}$ liegt in der metallischen Phase als Korund - Struktur vor, wobei die Vanadium - Ionen zu Paaren entlang der c-Achse geordnet sind. Jedes Vanadium - Ion ist oktaedrisch von sechs Sauerstoff - Ionen umgeben. Wechselt das System in die isolierende Phase, so verkippen die Vanadiumpaare gegenüber der $\mathrm{c}-$ Achse um $1,8^{\circ}$. Es findet eine strukturelle Umwandlung zu einer monoklin verzerrten Einheitszelle statt ${ }^{38}$.

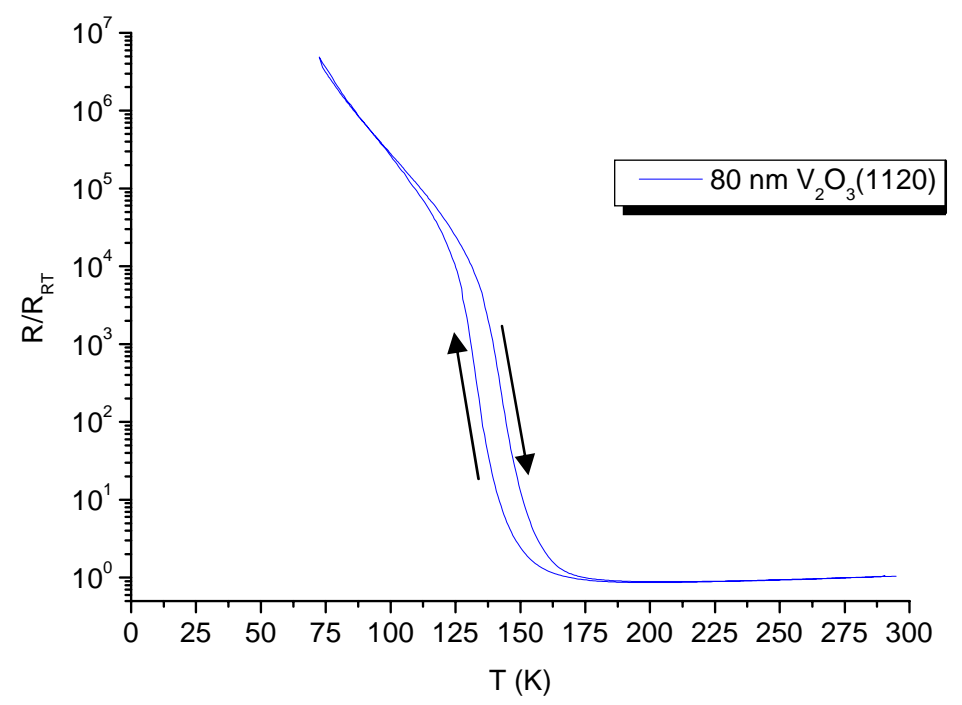

Abbildung 8.3: Transportmessung ${ }^{39}$ an einem $80 \mathrm{~nm}$ dicken Vanadiumsesquioxid - Film $\left(\mathrm{V}_{2} \mathrm{O}_{3}\right)$. Bei tiefen Temperaturen zeigt sich ein Kurvenverlauf, der von Halbleitern bekannt ist. Ab einer Temperatur von ca. $175 \mathrm{~K}$ liegt metallisches Verhalten vor. Die deutlich zu erkennende Hysterese zeigt, dass ein Phasenübergang 1. Ordnung vorliegt.

\footnotetext{
38 Weiterführende Informationen zu den theoretischen Grundlagen entnehmen Sie bitte [104].

${ }^{39}$ Diese Messung wurde von B. Sass, I. Physikalisches Institut, Universität Göttingen durchgeführt.
} 
Die im Folgenden dargestellten Messungen sind an einer gesputterten $\mathrm{V}_{2} \mathrm{O}_{3}-$ Probe ${ }^{40}$ mit einer Dicke von $80 \mathrm{~nm}$ aufgenommen worden. In Abbildung 8.3 ist der temperaturabhängige Transportwiderstand für diesen Film dargestellt. Entsprechend den Manganaten hängt auch hier die Übergangstemperatur stark von der Sauerstoffkonzentration und von mechanischen Spannungen ab $[105 ; 106]$. In einem Bereich von tiefen Temperaturen bis ca. $175 \mathrm{~K}$ zeigt sich isolierendes Verhalten, darüber lässt die in Abbildung 8.3 dargestellte $\mathrm{R}-\mathrm{T}$ - Charakteristik auf metallisches Verhalten schließen. Die dargestellte Messung wurde von Raumtemperatur zu tiefen Temperaturen hin durchgeführt. Anschließend ist der Widerstand zusätzlich beim Aufwärmvorgang der Probe gemessen worden. Deutlich ist eine Hysterese im mittleren Bereich zwischen $140 \mathrm{~K}$ und $150 \mathrm{~K}$ zu erkennen, was auf einen Phasenübergang 1. Ordnung hinweist.

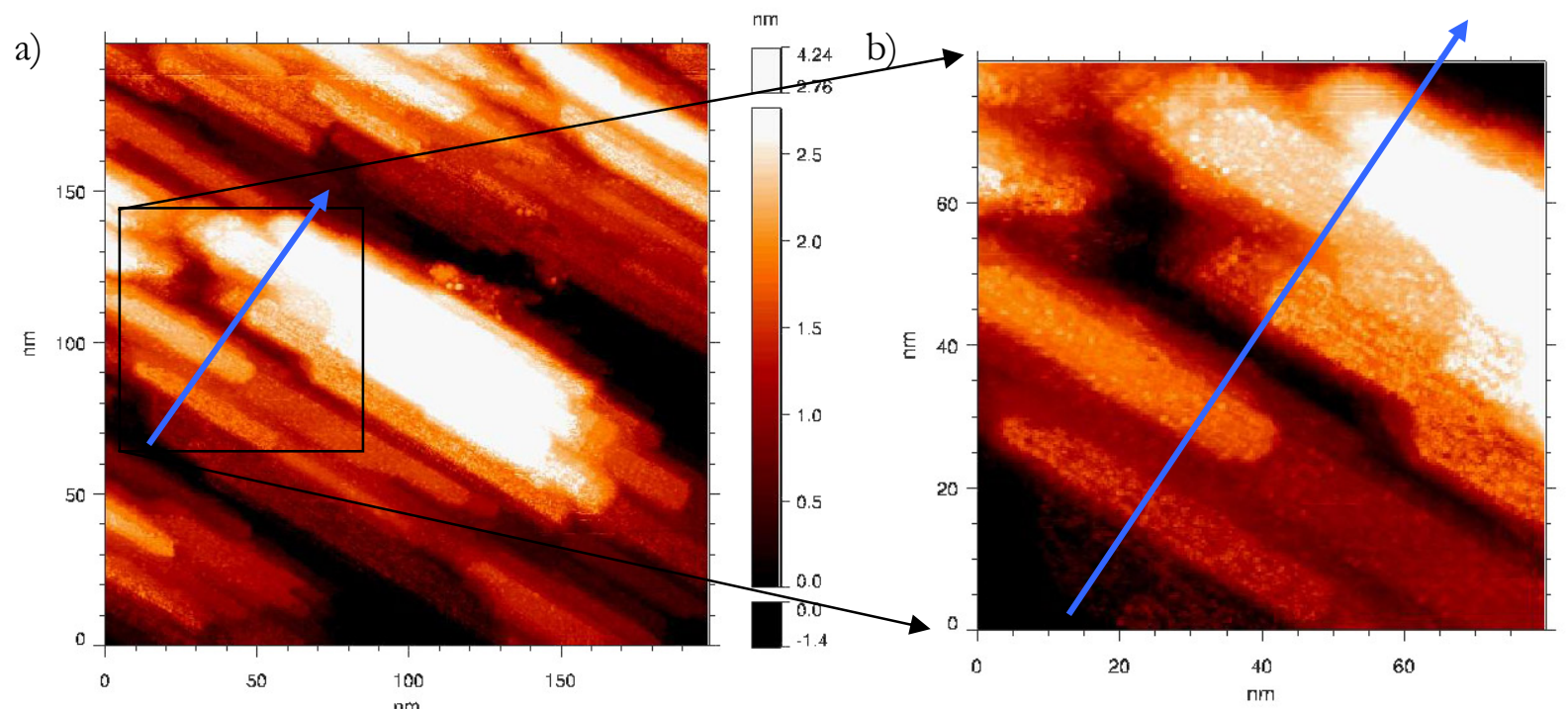

c)

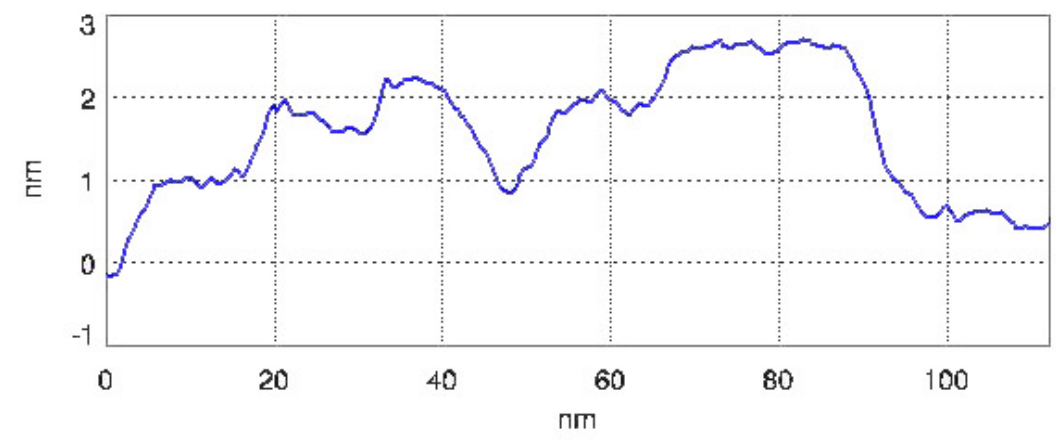

Abbildung 8.4: In (a) und (b) sind STM - Bilder von Vanadiumoxid gemessen bei Raumtemperatur dargestellt. (a) zeigt eine RMS - Rauhigkeit von $0.92 \mathrm{~nm}$. (b) ist eine Vergrößerung aus (a). Gut zu erkennen ist eine glatte Terassenstruktur. In (c) ist das Höhenprofil entsprechend des eingezeichneten blauen Pfeils aus (a) dargestellt.

${ }^{40}$ Die Probe ist von C. Tusche, I. Physikalisches Institut, Universität Göttingen präpariert worden. 
Es ist nun von Interesse, ob die in dieser Arbeit vorgestellte Mess- und Analysetechnik auch bei artverwandten Materialien, die einen Metall - Isolator - Übergang zeigen, angewandt werden kann. Dementsprechend wurden STS - und STM - Messungen bei Raumtemperatur und bei $100 \mathrm{~K}$ in der isolierenden Phase durchgeführt. Bei dem Transfer der Probe an Luft bildet sich eine Oxidschicht, die im Vakuum mittels Absputtern und Auslagern ${ }^{41}$ entfernt wurde. In Abbildung 8.4 ist eine Aufnahme bei Raumtemperatur dargestellt. Sie zeigt eine längliche Inselstruktur und weist eine RMS - Rauhigkeit von $0.92 \mathrm{~nm}$ auf. Eine Vergrößerung zeigt atomar glatte Oberflächen bestehend aus mehreren Nanometer großen Zellen, die entsprechend parallel verlaufender Perlenschnüre vorliegen. Dies wird durch einen Linescan quer zu der Inselstruktur in Abbildung 8.4 (c) verdeutlicht. Er zeigt deutliche Plateaus und Stufenhöhen von ca. 0,25 nm bzw. einem Vielfachen davon. Dieser Wert entspricht dem Ebenenabstand der (1120) - Ebenen [107].

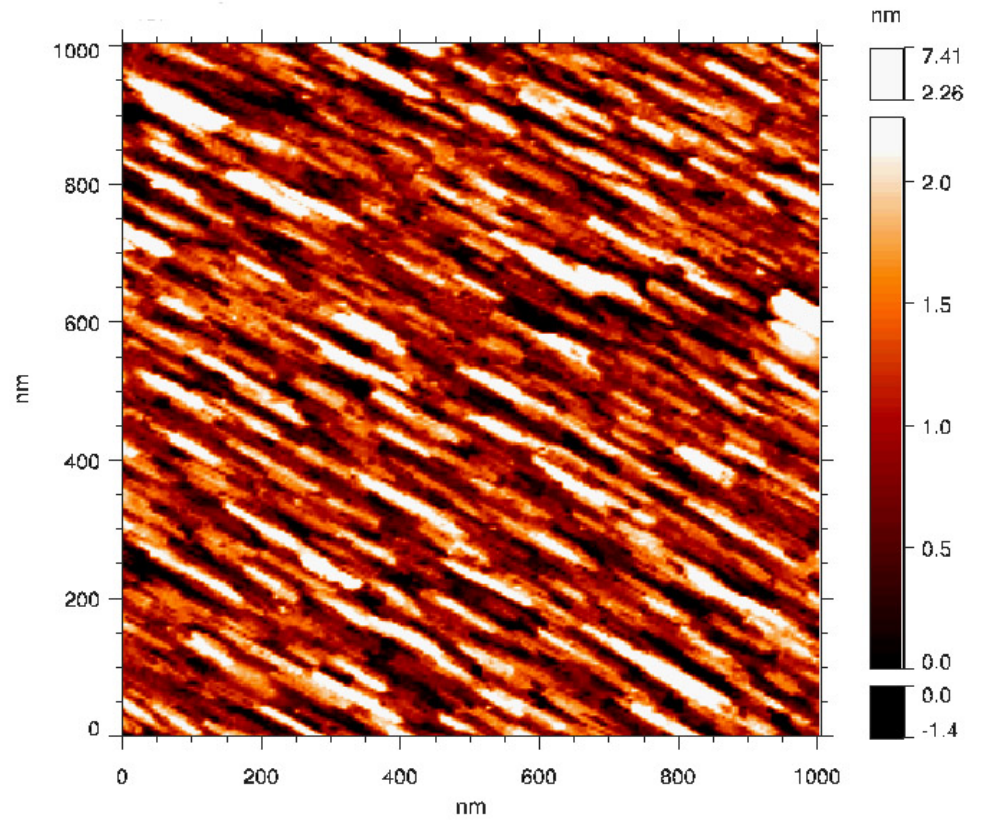

Abbildung 8.5: Dieses Bild zeigt die Topographie bei $T=100 \mathrm{~K}$. Es liegt eine vergleichbare Oberflächenstruktur zur Raumtemperaturmessung vor. Die RMS - Rauhigkeit lässt sich zu $0,75 \mathrm{~nm}$ bestimmen.

Das Bild aus Abbildung 8.5 zeigt die Messung bei $100 \mathrm{~K}$, also unterhalb des MIT, in einem größeren Ausschnitt. Ein Vergleich mit der Raumtemperaturmessung aus Abbildung 8.4 zeigt die gleiche Oberflächenstruktur. Die RMS-Rauhigkeit zeigt im Vergleich zur Raumtemperaturmessung einen vergleichbaren Wert von $0,75 \mathrm{~nm}$, wobei hier aufgrund der größeren Fläche von einer verbesserten Statistik ausgegangen werden kann.

${ }^{41} 600^{\circ} \mathrm{C}$ für eine Stunde 


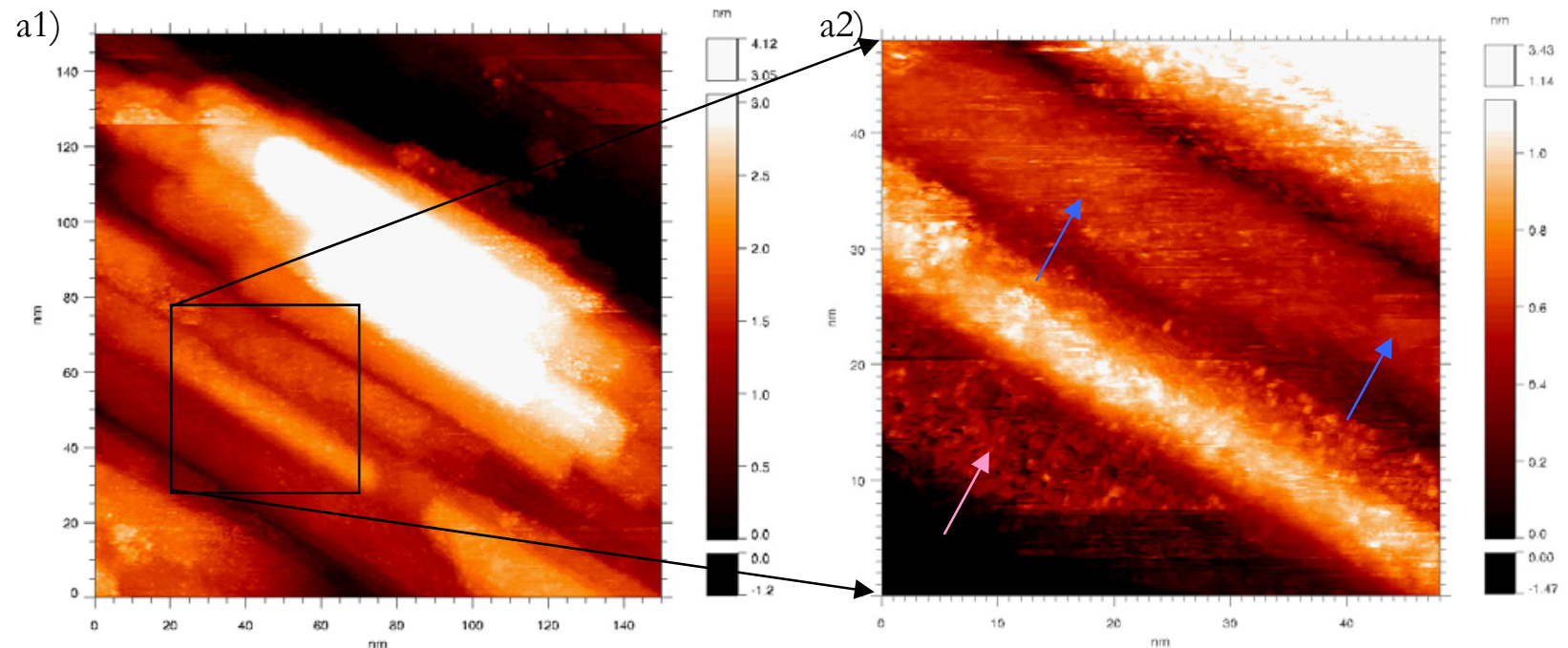

b1)

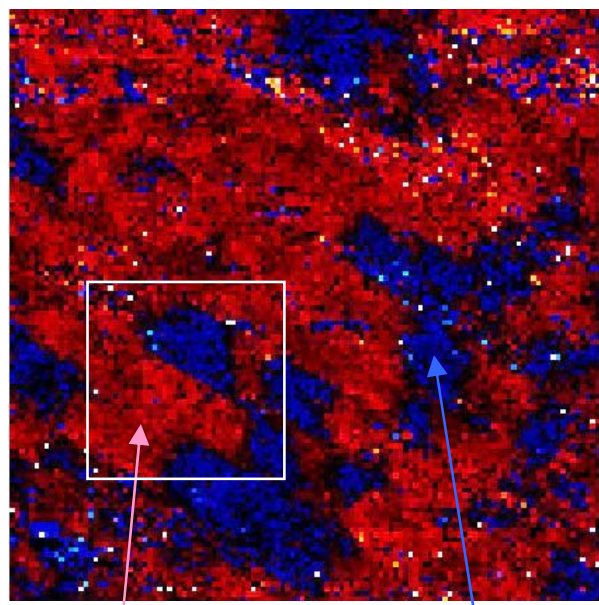

leitfähig

c1)

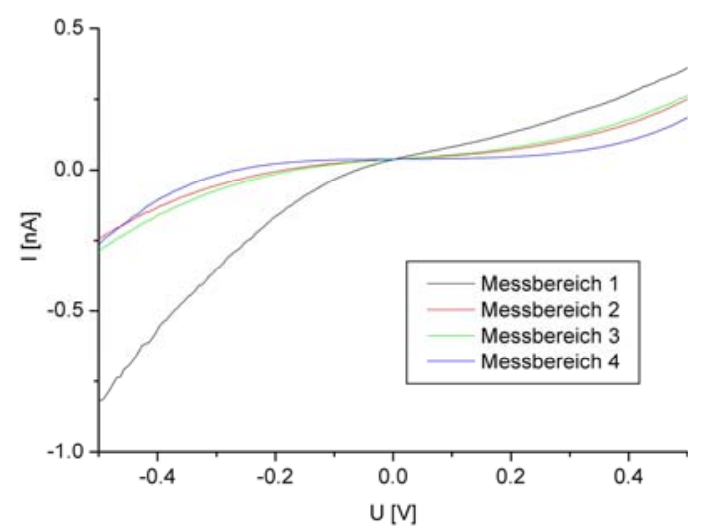

b2)

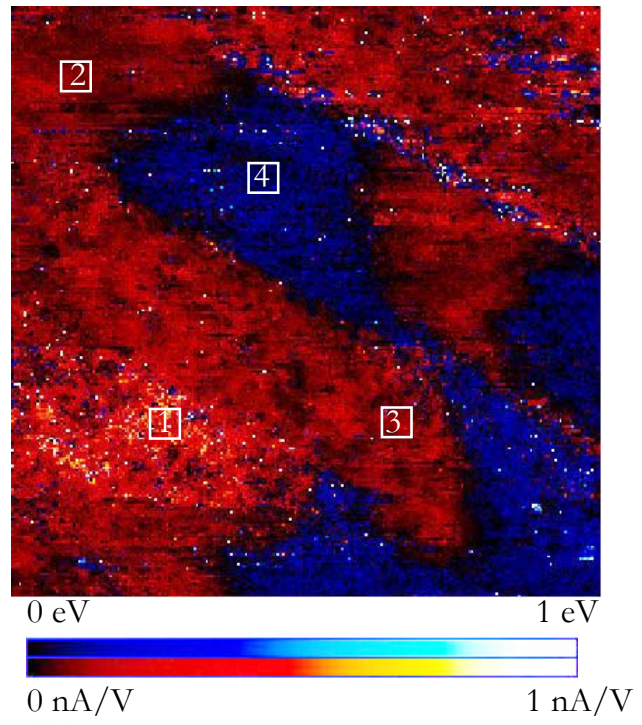

c2)

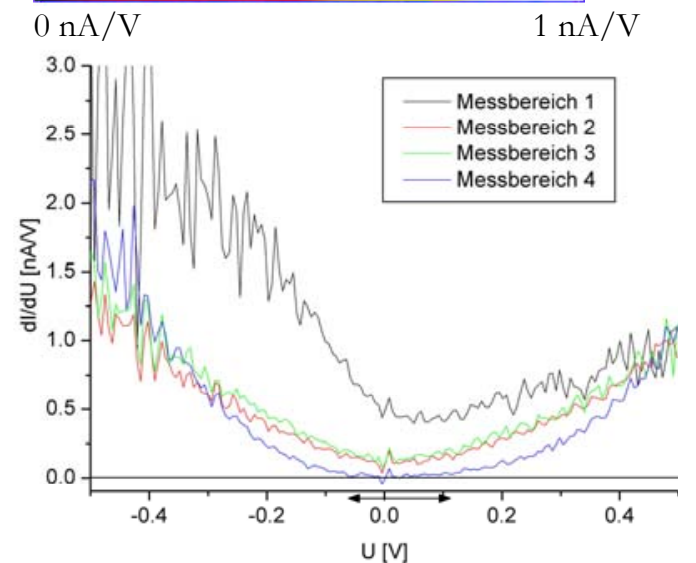

Abbildung 8.6: Die Bilder in (a1) und (a2) zeigen topographische Darstellungen der Vanadiumoxid Oberfläche Mit einem Scanbereich von $(150 \mathrm{~nm})^{2}$ bzw. $(48 \mathrm{~nm})^{2}$ gemessen bei Raumtemperatur. Das Bild aus (a2) entspricht einer Vergrößerung der in (a1) gekennzeichneten Region. In (b1) und (b2) sind die zu den topographischen Bildern gehörigen Leitfähigkeits- bzw. Isolationskarten dargestellt. Kennlinien und deren Ableitungen von je $10 \times 10$ Messpunkten aus verschiedenen Bereichen in (b2) sind in (c1) und (c2) zu finden. 
Zusätzlich zu den topographischen STM-Bildern sind spektroskopische STS-Messungen durchgeführt worden. Die Ergebnisse bei $T=300 \mathrm{~K}$ sind in Abbildung 8.6 dargestellt. Die Topographien (a1) und (a2) besitzen einen Größe von $150 \mathrm{~nm} \times 150 \mathrm{~nm}$ bzw. $48 \mathrm{~nm} \times 48 \mathrm{~nm}$, wobei (a2) eine Vergrößerung aus (a1) darstellt. Parallel zu den Bildern sind gerastert Kennlinien aufgezeichnet worden und mithilfe der in Kapitel 4.1 vorgestellten Methode analysiert worden. Diese Ergebnisse sind in den Abbildungen (b1) und (b2) dargestellt und besitzen die gleiche Dimension der Oberflächendarstellungen aus (a). Beide Bilder zeigen Leitfähigkeit (rot dargestellt), die stellenweise von isolierenden Bereichen (blau) unterbrochen wird. Dieses Ergebnis entspricht nur teilweise den Erwartungen, da die Transportmessung aus Abbildung 8.3 einen scharfen Phasenübergang 1. Ordnung bei $T=175 \mathrm{~K}$ zeigt und somit bei Raumtemperatur nur noch eine homogene, leitfähige Phase vorliegen sollte. Eine genauere Betrachtung in Abbildung (b2) zeigt jedoch drei verschieden leitfähige Gebiete:

Isolation: blau dargestellt

Leitfähigkeit: dunkelrot dargestellt

Erhöhte Leitfähigkeit: hellrot dargestellt

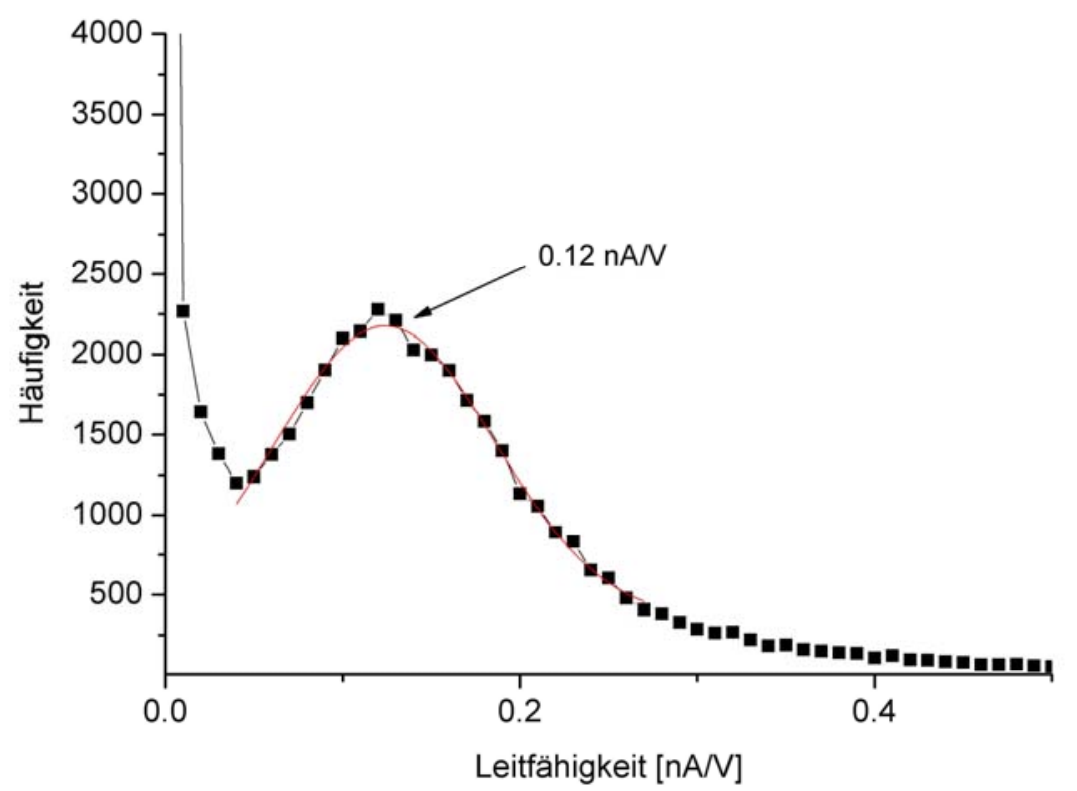

Abbildung 8.7: Häufigkeitsverteilung für Messung dargestellt in Abbildung 8.6 (b2) bei Raumtemperatur. Die leitfähige Phase zeigt ihr Maximum bei $1,2 \cdot 10^{-1} n A / V$. Dieser Wert wurde durch einen Gaußfit bestimmt (dargestellt als rote Kurve). 
Innerhalb jeder dieser Bereiche sind $10 \times 10$ Kennlinien betrachtet (siehe nummerierte Rechtecke in (b2)) und als gemittelte Kennlinien und deren Ableitungen in (c1) und (c2) aufgetragen worden. Die Bereiche „2“ und „3“ zeigen vergleichbare Kennlinien und eine Leitfähigkeit von ca. 1,2 $10^{-1} n A / V$. Dies ist mit dem Ergebnis der Häufigkeitsverteilung aus Abbildung 8.7 identisch. Dort zeigt sich ein Peak - Maximum der am häufigst vorkommenden Leitfähigkeit bei 1,2 $10^{-}$ ${ }^{1} n A / V$. Ein direkter Vergleich mit Literaturwerten aus STS - Messungen zeigt qualitativ eine gute Übereinstimmung $\left(\sim 3,0 \cdot 10^{-2} \mathrm{nA} / \mathrm{V}\right.$ aus [108]). Somit ist davon auszugehen, dass es sich hierbei um die zu erwartende, leitfähige Phase handelt. Eine genauere Betrachtung der zwei weiteren Bereiche „1“ (erhöhte Leitfähigkeit: $~ 4,0 \cdot 10^{-1} \mathrm{nA} / \mathrm{V}$ ) und „4“ (Auftreten einer Bandlücke: $\sim 0,17 \mathrm{eV})$ lässt den Schluss zu, dass es sich hierbei um Adsorbate oder Fremdphasen $\left(\mathrm{VO}_{\mathrm{x}}\right)$ an der Oberfläche handelt. Dort zeigt die Topographie in Abbildung 8.6 (a2) eine erhöhte Rauhigkeit (siehe Pfeile). Eine mögliche Erklärung für das Vorliegen von Adsorbaten kann ein zu kurz gewählter Sputterprozesses sein. Bei dem Transport der Probe an Luft bildet sich an der Oberfläche $\mathrm{VO}_{2}$ mit einem Übergang bei $340 \mathrm{~K}$ [109; 110]. Falls diese Phase noch stellenweise vorliegt, ist mit isolierenden Bereichen zu rechnen. Eine weitere Möglichkeit, die Anwesenheit der isolierenden Bereiche weit weg vom Übergang zu erklären, stellt die Existenz von dimerisierten und undimerisierten Oberflächenbereichen des $\mathrm{V}_{2} \mathrm{O}_{3}$ dar. Entsprechend [111] kann eine Überdotierung an Vanadiumatomen auf der Oberfläche zu isolierenden Bereichen führen. Bemerkenswert ist, dass die unterschiedlich leitfähigen Bereiche mit den Kornstrukturen korrelieren. Hierfür können auftretende Phasenseparationen weiteres Erklärungspotential liefern.
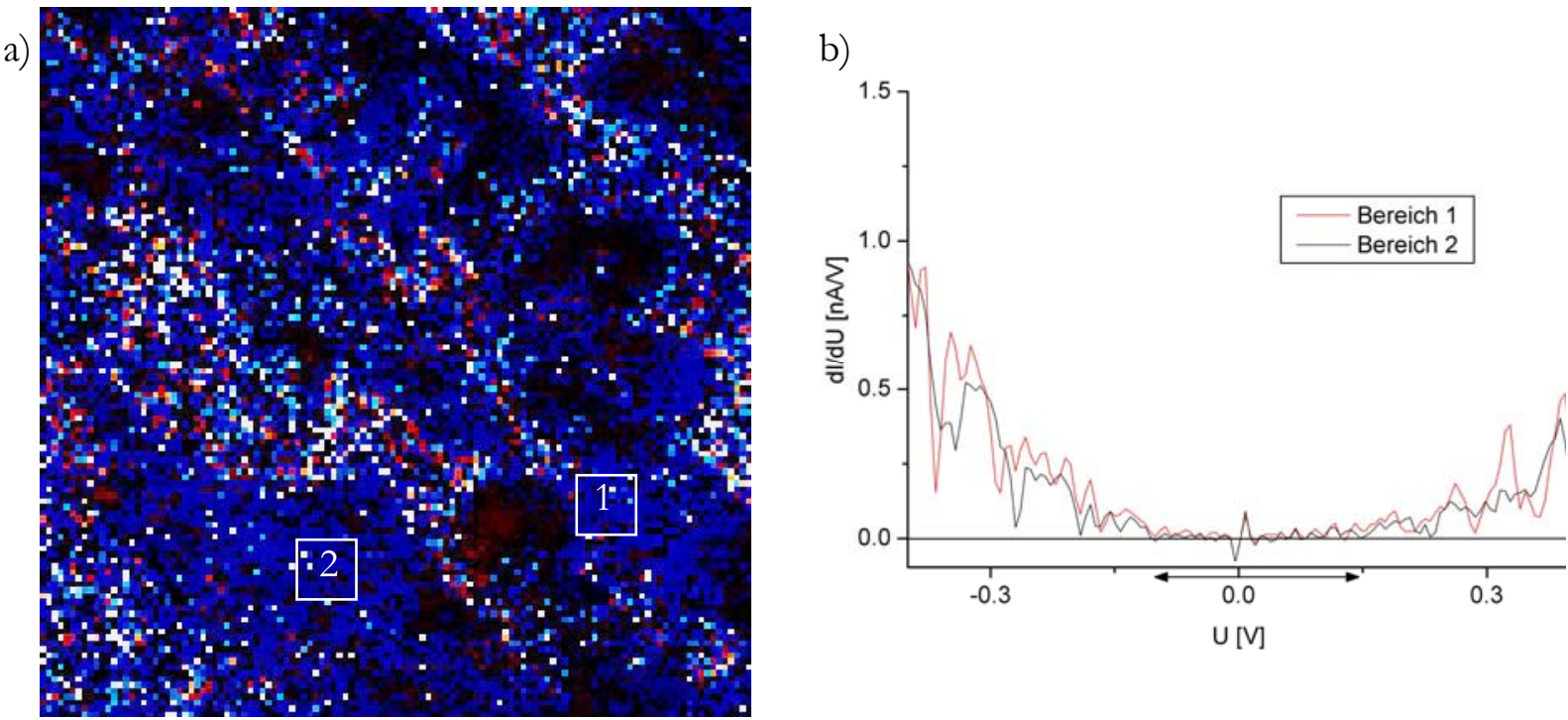

Abbildung 8.8: In (a) ist die Leitfähigkeits- bzw. Isolationskarte mit einer Größe von $(150 \mathrm{~nm})^{2}$ aufgenommen bei $T=100 \mathrm{~K}$ dargestellt. Bereiche von $10 \times 10 \mathrm{Kennlinien}$ sind gemittelt worden und in (b) als Ableitung dargestellt. 
Eine weitere, vergleichbare Messung ist bei $100 \mathrm{~K}$ - unterhalb des Übergangs - durchgeführt worden. Die Leitfähigkeitsverteilung für einen Bereich von $150 \mathrm{~nm} \times 150 \mathrm{~nm}$ ist in Abbildung 8.8 (a) dargestellt und zeigt ein hauptsächlich isolierendes Verhalten der Probe. Entsprechend der oben beschriebenen Vorgehensweise sind Kennlinien über Bereiche von $10 \times 10$ Messpunkten gemittelt worden und in Abbildung 8.8 (b) als Ableitung aufgetragen. Sie zeigen eine Bandlücke mit einer Breite von ca. $0.25 \mathrm{eV}$ (Literaturwert aus [109]: 0,45 $55 \mathrm{eV}$ ).

Zusammenfassend zeigen diese Ergebnisse qualitativ Übereinstimmung mit Resultaten aus der Literatur. Die untersuchte Vanadiumoxidprobe hat bei Raumtemperatur hauptsächlich metallisches Verhalten gezeigt, wohingegen unterhalb des Übergangs vornehmlich Isolation anzutreffen ist.

\subsection{Ergebnisse am $\mathrm{K}-(\mathrm{BETS})_{2} \mathrm{FeCl}_{4}-$ Einkristall}

Als ein weiteres artfremdes Material wurde nach der $\mathrm{V}_{2} \mathrm{O}_{3}$-Schicht aus Kapitel 8.2 ein $\kappa$ (BETS) ${ }_{2} \mathrm{FeCl}_{4}-$ Einkristall $^{42}$ gewählt. Es handelt sich hierbei um einen organischen Einkristall mit metallischen Eigenschaften. Seit Anfang der 50er Jahre zeigt die Forschung aufgrund der interessanten elektrischen Eigenschaften eine besondere Aufmerksamkeit für diese Materialklasse. Beginnend über anfängliche Ergebnisse metallischer Leitfähigkeit wurde 1981 der erste organische Supraleiter gefunden[112]. Aufgrund seines Entdeckers nennt man diese Stoffe Becchgaard - Salze.

Zwei temperaturabhängige Transportmessungen an einem $\kappa$-(BETS) ${ }_{2} \mathrm{FeCl}_{4}-$ Einkristall in Schichtebene (parallel zur Kristalloberfläche (1) und senkrecht zu den Schichten (durch den Kristall hindurch (2) sind in Abbildung 8.9 (a) gezeigt. In Abbildung (b) ist die Stromrichtung der Messungen an der Kristallstruktur illustriert und verdeutlicht den erhöhten Transportwiderstand senkrecht zu den Ebenen aufgrund der blau eingezeichneten isolierenden Kristallebenen ${ }^{43}$. Alle im Folgenden vorgestellten Messergebnisse sind parallel zu den Schichten aufgenommen worden. Dementsprechend ist die Transportmessung (1) aus Abbildung (a) für weiterführende Diskussionen relevant. Um einen Stromtransport innerhalb der leitfähigen Ebene zu begünstigen und Einflüsse von isolierenden Schichten zu reduzieren, ist das Probenplättchen schräg im Leitsilber, das zur Kontaktierung genutzte wurde, befestigt worden (siehe Abbildung 8.10).

\footnotetext{
${ }^{42}$ Der Einkristall wurde von N. Toyota, Physics Department, Graduate School of Science, Tohoku University, Japan zur Verfügung gestellt.

${ }^{43}$ Weiterführende Informationen entnehmen Sie bitte aus [113; 114]
} 


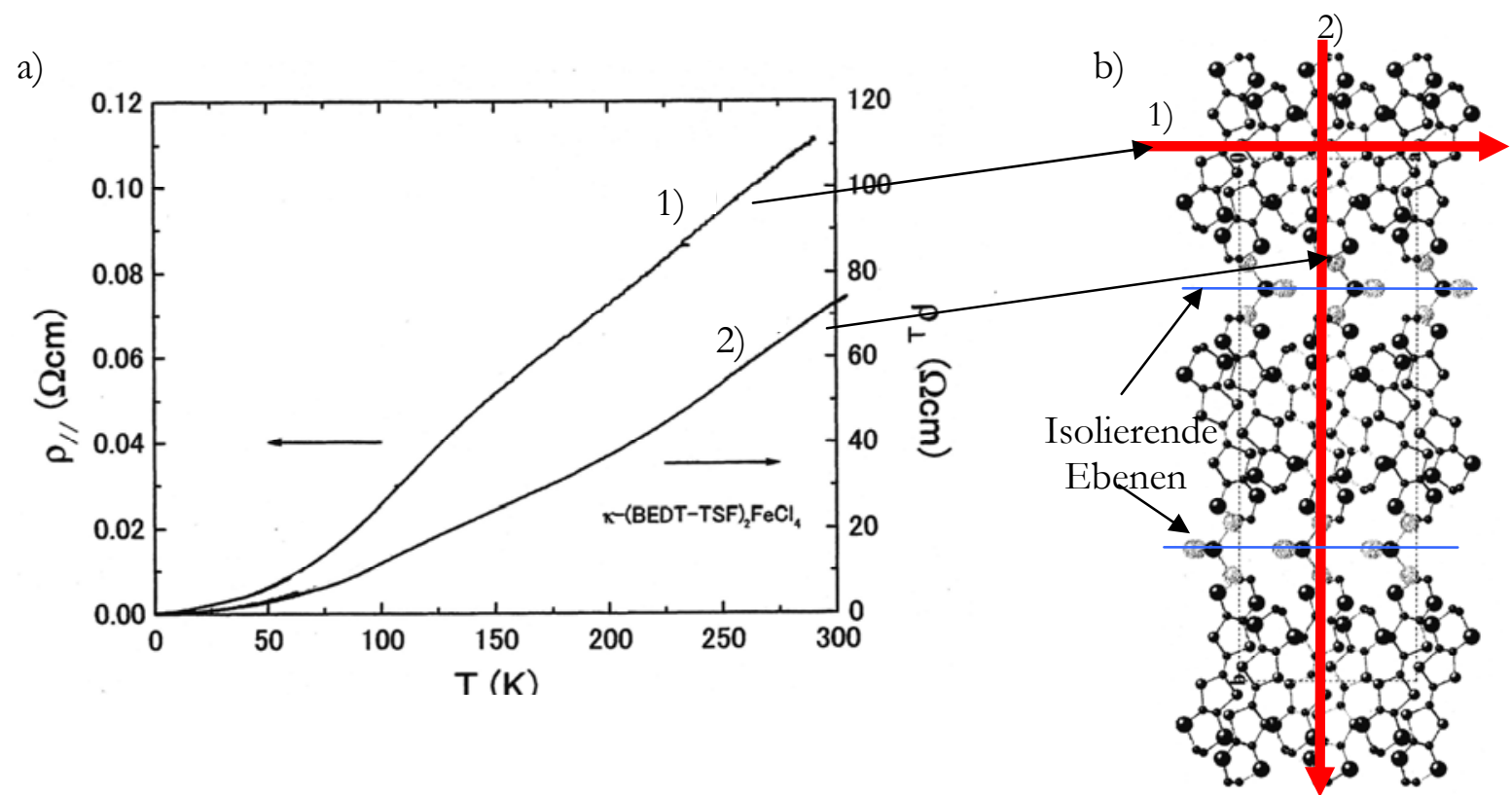

Abbildung 8.9: In (a) ist der temperaturabhängige Widerstand für die Messungen in der Ebene und senkrecht zu den Ebenen aufgetragen. (b) skizziert die Kristallstruktur und die Messrichtungen aus (a) [113]

In Abbildung 8.11 sind zwei Topographien, aufgenommen bei $114 \mathrm{~K}$, abgebildet. Das Bild (a) zeigt einen Ausschnitt mit einer Größe von $500 \times 500 \mathrm{~nm}^{2}$ und besitzt eine RMS - Rauhigkeit von 0,68 nm. Diese Form der Morphologie wurde bei fast allen durchgeführten Messungen gefunden - größtenteils liegt eine sehr glatte Oberfläche vor. Eine der wenigen Ausnahmen ist in (b) dargestellt. Hier zeigt sich ein stark zerklüftetes Gebilde mit einer Höhendifferenz von ca. $100 \mathrm{~nm}$ bei gleicher Ausschnittsgröße. Beide Bilder besitzen eine Auflösung von $800 \times 800$ Bildpunkten, wobei bei jedem 5ten Bildpunkt eine Kennlinie aufgenommen wurde.

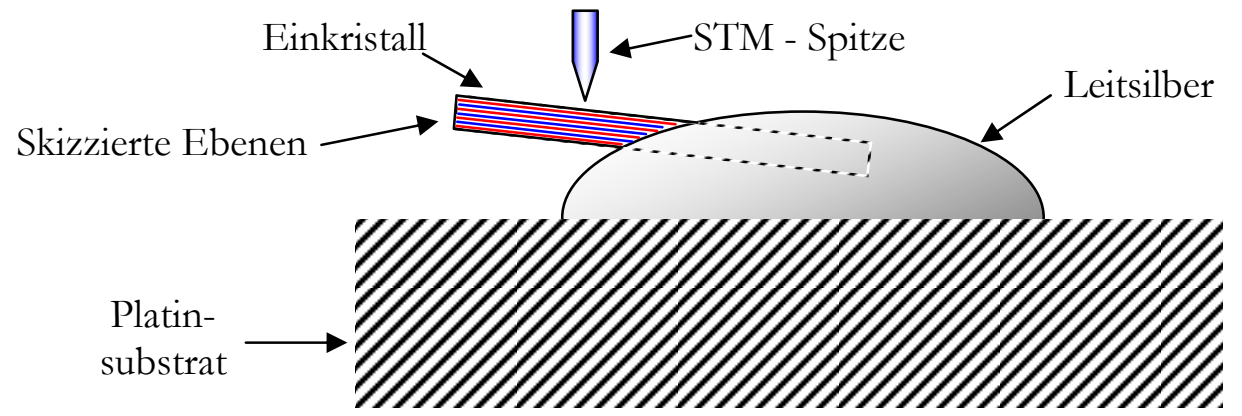

Abbildung 8.10: Skizzierte Probenkontaktierung und Befestigung 
a)

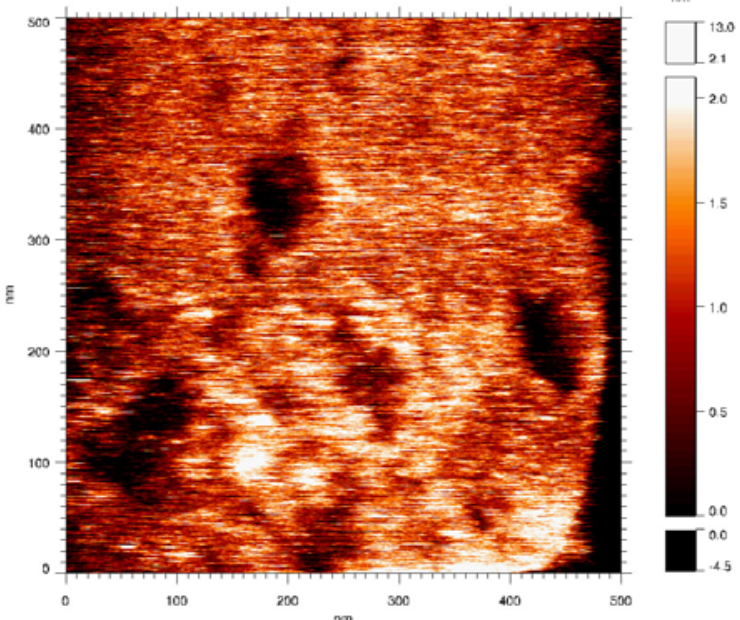

b)

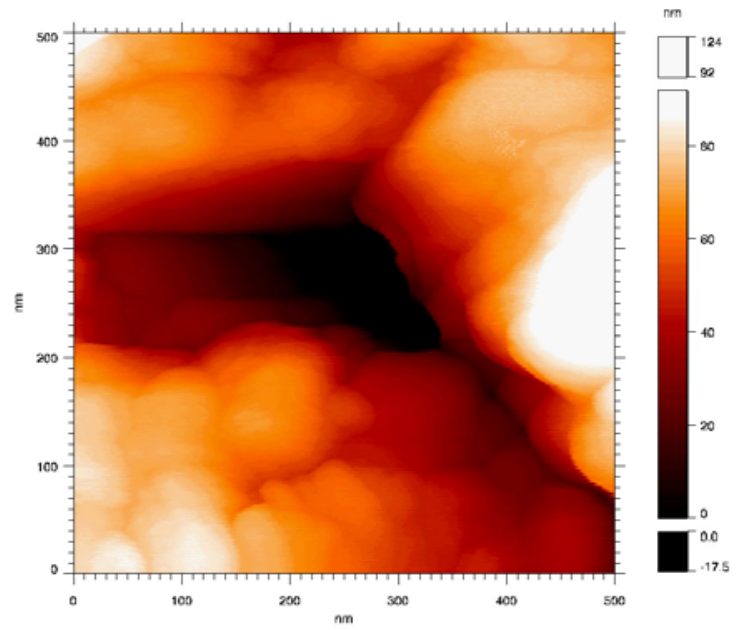

Abbildung 8.11: STM - Bilder gemessen am $\kappa-(\mathrm{BETS})_{2} \mathrm{FeCl}_{4}$ - Einkristall bei $114 \mathrm{~K}$. Am häufigsten ist eine sehr glatte Oberfläche entsprechend Abbildung (a) anzutreffen. Stellenweise existieren aber auch größere Unebenheiten (b).

Die Ergebnisse der gerasterten Kennlinienanalyse sind temperaturabhängig in Abbildung 8.12 für $77 \mathrm{~K}, 114 \mathrm{~K}$ und $300 \mathrm{~K}$ dargestellt. Alle drei Bilder weisen eine sehr homogene Darstellung auf, wobei eine Korrelation mit der Oberfläche, bzw. eine Form von elektronischer Phasenseparation nicht $\mathrm{zu}$ erkennen ist. Die leitfähigen (rot) und isolierenden (blau) Bereiche scheinen nicht voneinander getrennt vorzuliegen. Generell überwiegt bei allen Temperaturen die blau dargestellte, anscheinend isolierende Phase. Ob es sich hierbei um Ergebnisse an leitfähigen oder isolierenden Schichtebenen handelt, kann nicht abschließend geklärt werden. Die niedrige Bildschärfe aus Abbildung 8.11 (a) deutet jedoch auf eine isolierende Schicht an der Probenoberfläche hin, die den Tunnelstrom zu leitfähigen Ebenen behindert.

Der visuelle Eindruck der Bilder aus Abbildung 8.12 zeigt, dass die Blauwerte sich kaum bei den verschiedenen Temperaturen ändern. Dies wird von dem Histogramm in (c) gestützt, das die Häufigkeitsverteilung der gemessenen Gapbreiten für $77 \mathrm{~K}$ und Raumtemperatur zeigt. Beide Graphen unterscheiden sich in ihrer Höhe, die Maxima sind jedoch kaum voneinander verschoben und liegen bei ca. 0,27 eV. Das bedeutet, dass nur die Häufigkeit der isolierend verlaufenden Kennlinien mit höherer Temperatur zugenommen hat - die Gapbreite bleibt unverändert. Eine Betrachtung der Leitfähigkeiten bei unterschiedlicher Temperatur ist in Abbildung (b) dargestellt. Bei tiefer Temperatur verläuft die Häufigkeitsverteilung für $77 \mathrm{~K}$ deutlich höher als bei Raumtemperatur - es existieren also mehr metallisch verlaufende Kennlinien bei tiefer Temperatur. Deutlich ist ein entgegengesetztes Verhalten zwischen den beiden Histogrammen (b) und (c) zu beobachten, dass auf eine erhöhte Leitfähigkeit bei tiefen Temperaturen hindeutet. Dementsprechend ist bei Raumtemperatur ein höherer Widerstand zu erwarten. 

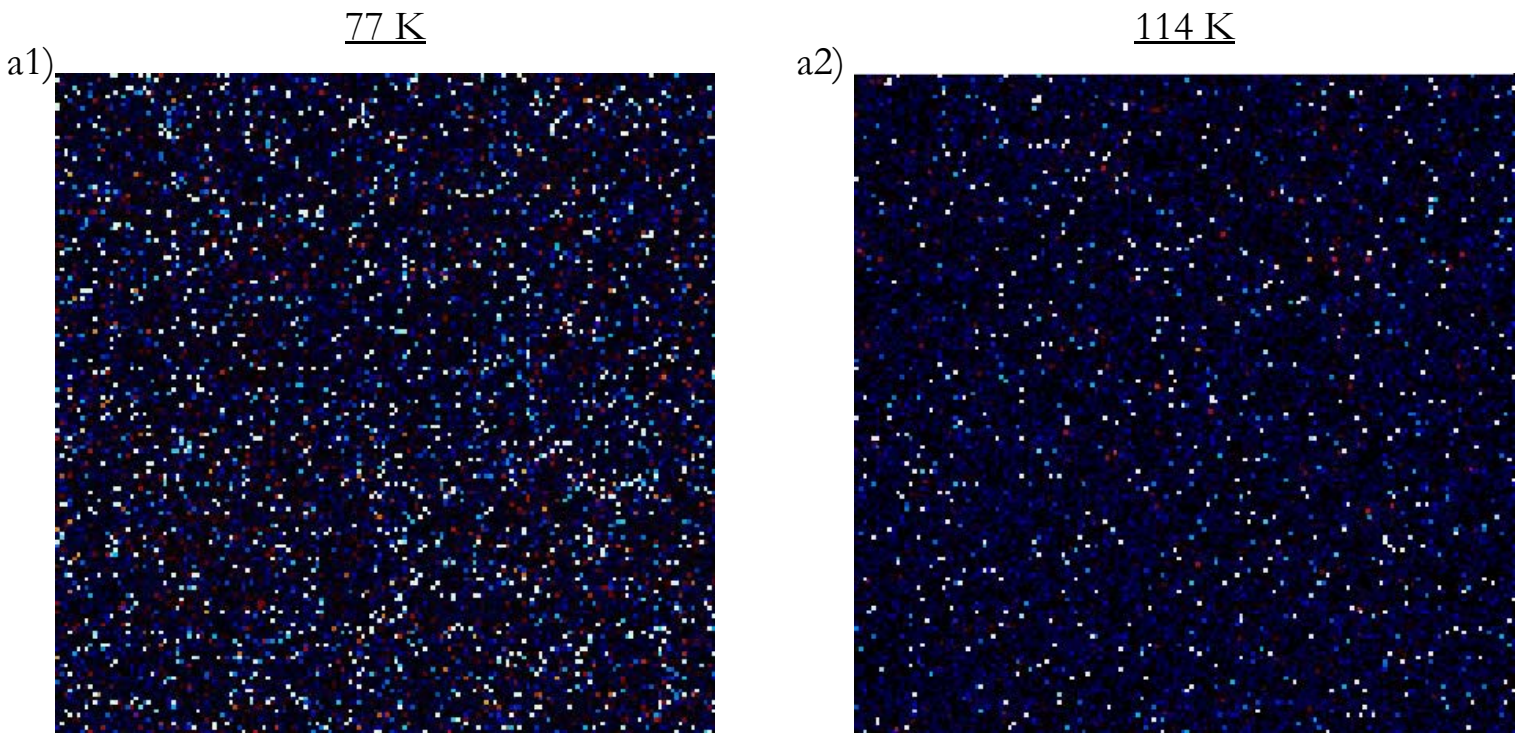

a3)

$\underline{300 \mathrm{~K}}$
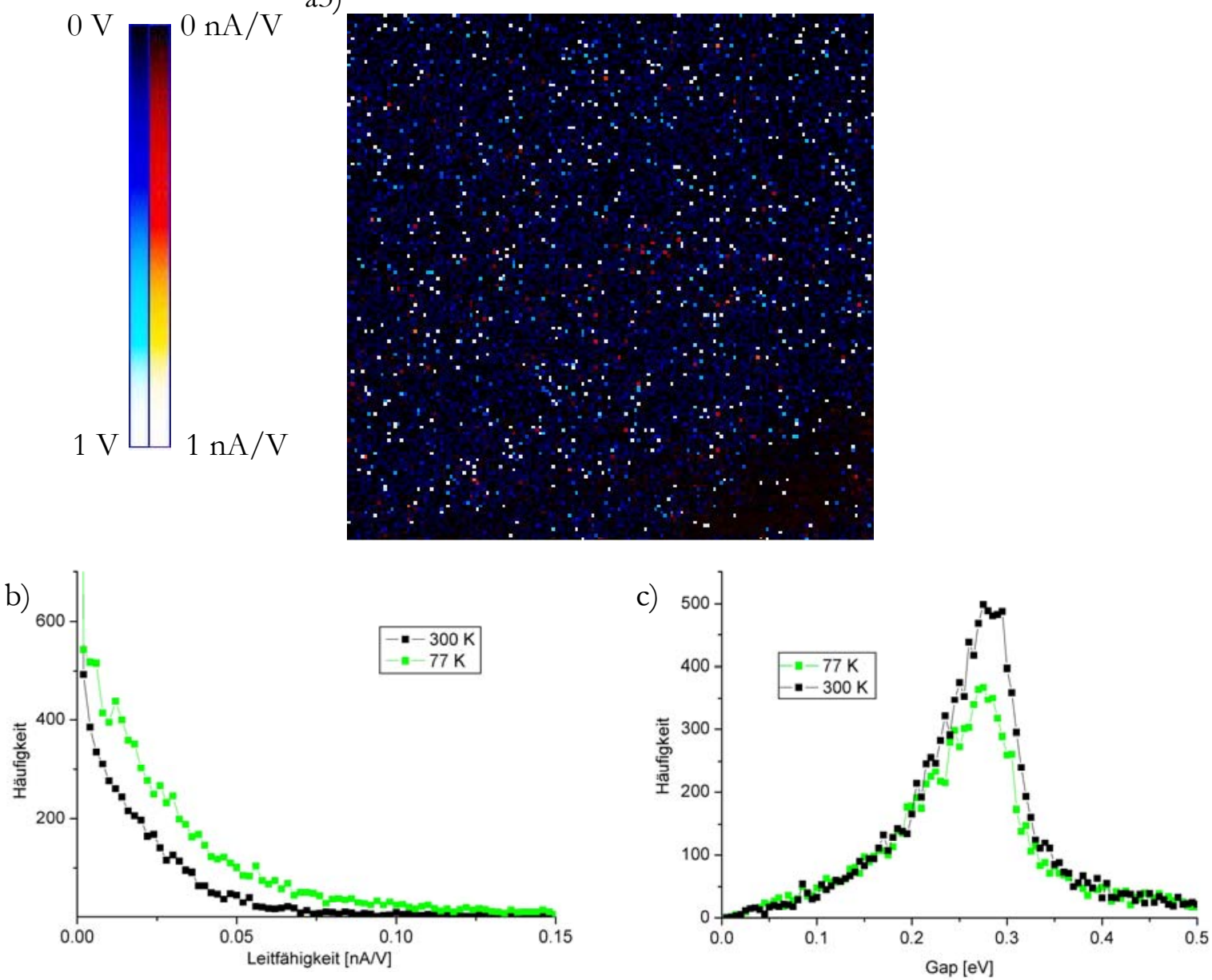

Abbildung 8.12: In den Abbildungen (a1) bis (a3) sind kombinierte Leitwert- und Gapkarten für verschiedene Temperaturen dargestellt. Häufigkeitsverteilungen für Leitfähigkeiten bzw. Bandlückenbreiten gemessen bei $77 K$ und Raumtemperatur sind in (b) und (c) aufgetragen. 
Die Leitwert- bzw. Isolationskarten aus Abbildung 8.12 dienen für die Auftragung in Abbildung 8.13 als Grundlage für durchgeführte Netzwerksimulationen. Die Resultate der Kalkulationen sind als blaue Sterne dargestellt. Die Daten des normierten spezifischen Transportwiderstands (rot gezeichnet) sind an einem identischen Einkristall gemessen worden und stammen entsprechend Abbildung 8.9 (a-1) aus $[113]^{44}$. Ein direkter Vergleich der Ergebnisse zeigt tendenziell eine recht gute Übereinstimmung.

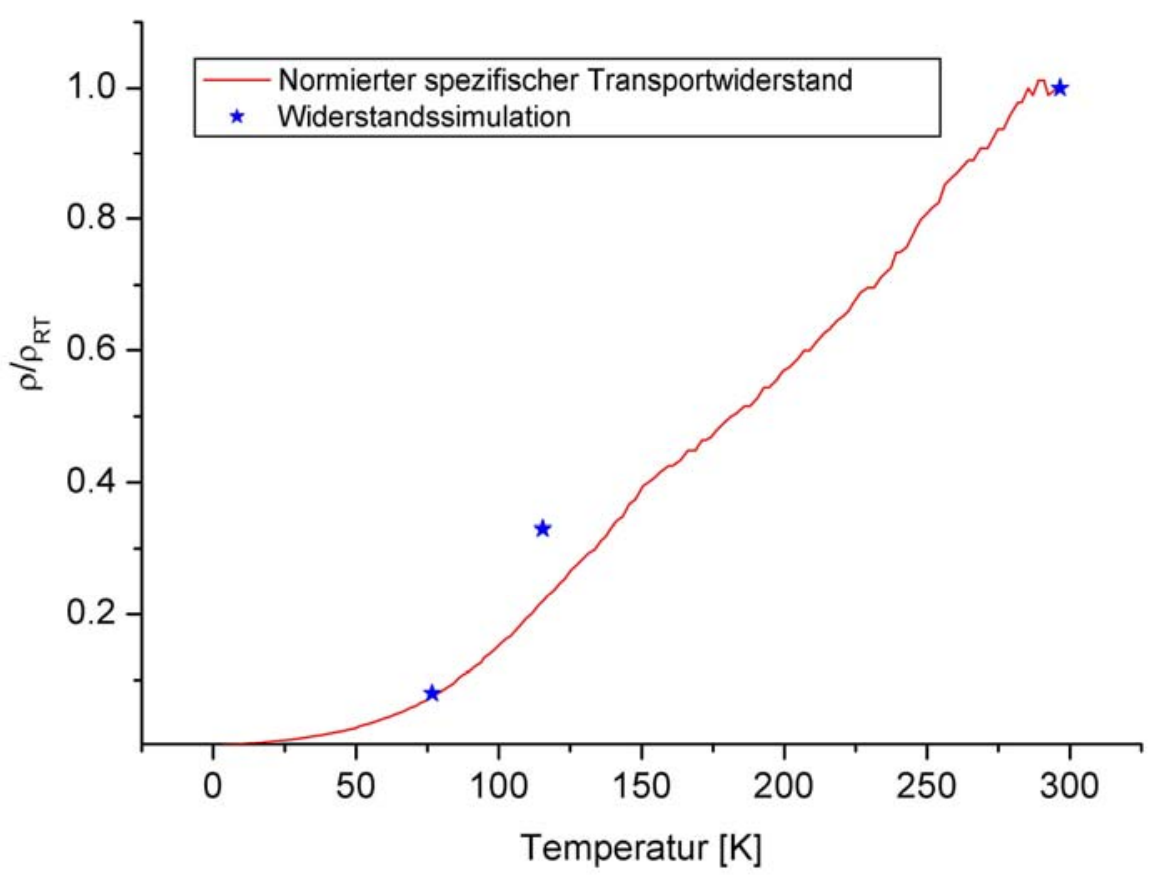

Abbildung 8.13: Der Transportwiderstand und die Ergebnisse aus den Widerstandssimulationen sind normiert gegen die Temperatur aufgetragen.

${ }^{44}$ Die Originaldatensätze standen nicht zur Verfügung, dementsprechend wurde der Graph im Original gescannt und mit dem Softwareprodukt Grafula3 V1.13 aus dem Hause WESiK Soft HaUs digital umgesetzt. 


\subsection{Wirtschaftliche Analyse und technische Anwendungsgebiete von magnetoresistiven Materialien}

Seit der Entdeckung des GMR ${ }^{45}$ - Effektes im Jahre 1988 [115] besitzen mangnetoelektronische Systeme in der Forschung und Wirtschaft eine wachsende Bedeutung. Mit dieser Entdeckung war es von nun an möglich, den Stromfluss gezielt mit magnetischen Feldern zu steuern. Somit werden Elektronen in der Informationstechnologie nicht mehr nur als Träger der elektrischen Ladung angesehen, sondern sind nun auch Träger eines magnetischen Moments zu betrachten. Die daraus resultierenden, breit gefächerten technologischen Einsatzmöglichkeiten und die ungewöhnlichen Kombinationen von Eigenschaften zeigen das hohe Potential der Magnetoelektronik. Eine Verknüpfung der physikalischen Wechselwirkung mit ihren Einsatzmöglichkeiten ist in Abbildung 8.14 dargestellt.

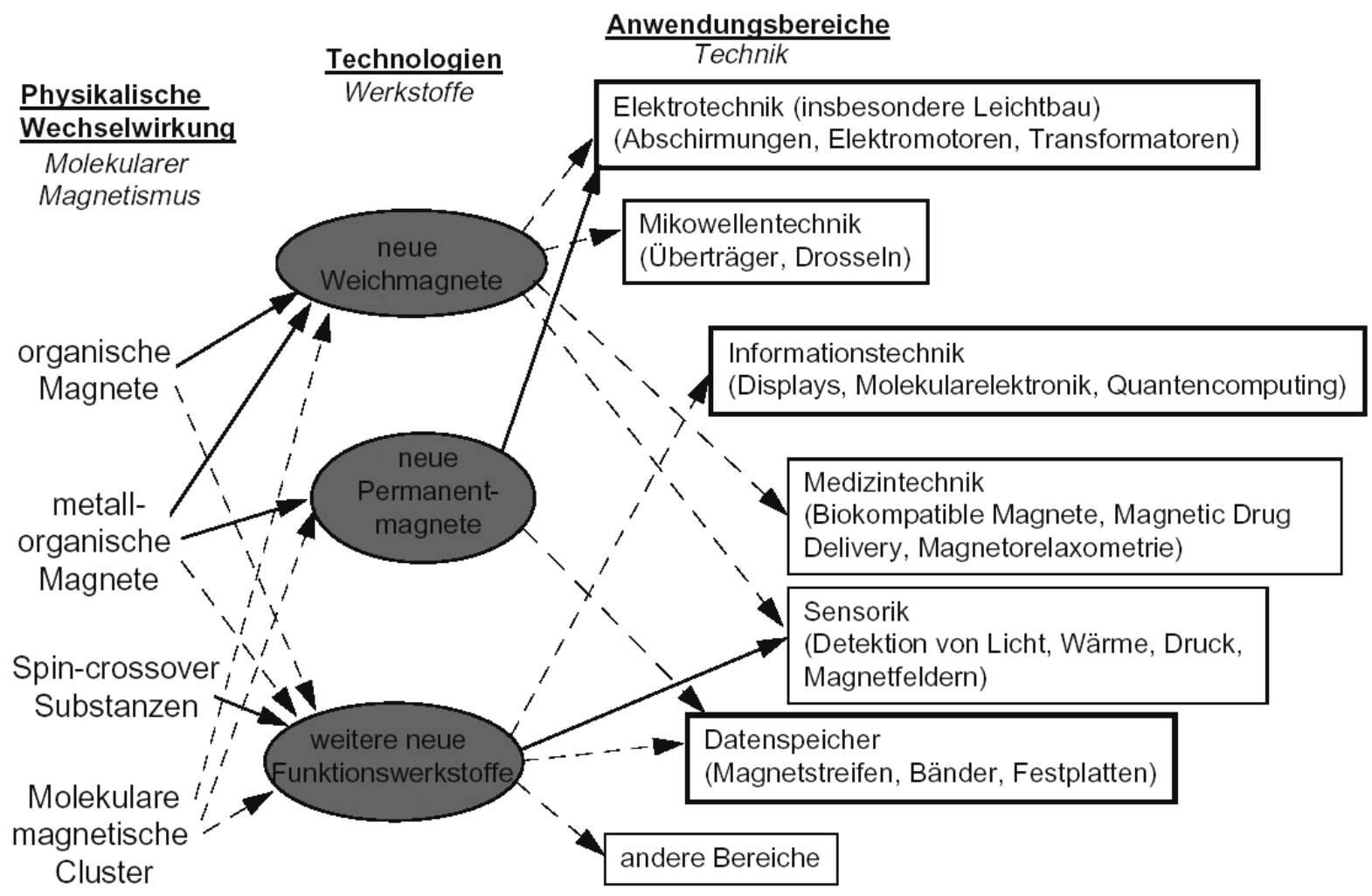

(C) VDI-Technologiezentrum

Abbildung 8.14: Schematische Darstellung für die Verknüpfung von physikalischen Wechselwirkungen und ihren möglichen Anwendungsgebieten. Gestrichelte Pfeile sollen andeuten, dass ein Innovationspotential besteht. Aus [116]

45 Giant MagnetoResistive 
Deutlich ist die Mannigfaltigkeit der Möglichkeiten zu erkennen, wobei die technologische Umsetzung bisher noch nicht vollkommen ausgeschöpft ist. Nach dem heutigen Entwicklungsstand können magnetoelektronische Bauelemente in folgende Einsatzgebiete aufgeteilt werden:

Sensorik (z.B. Fahrdynamiksensoren, Drehratensensoren und Festplattenleseköpfe) Speicherbauelemente

Spin - Transistoren

Sensoren besitzen die Möglichkeit, magnetische oder magnetisch kodierte Informationen in elektrische Signale umzuwandeln. Sie sind in der Lage, eine Vielzahl von mechanischen Parametern zu erfassen, wodurch ihre Anwendungsgebiete von der Fahrzeugtechnik über den Maschinenbau bis hin zur Medizintechnik reichen. Für die Nutzung von GMR - Sensoren spricht das sehr große Ausgangssignal, so dass auf eine aufwendige Vorverstärkung des Signals gegebenenfalls verzichtet werden kann. Mit dem hohen Ausgangssignal ist zusätzlich eine sehr große Empfindlichkeit verbunden. Erste kommerzielle GMR - Sensorsysteme sind seit kurzem von den Firmen HY-Line Sensor Tec. und Siemens erhältlich. Ein Vergleich der unterschiedlichen Technologien ist in Abbildung 8.15 aufgetragen.

$\begin{array}{lcccc}\text { Eigenschaften } & \text { GMR } & \text { AMR } & \text { Hall } & \text { Induktiv } \\ \text { Temperaturstabilität } & +++ & ++ & + & ++ \\ \text { Ausgangssignal } & +++ & ++ & + & \text { größenabh. } \\ \text { Empfindlichkeit } & +++ & +++ & ++ & ++ \\ \text { Leistungsverbrauch } & +++ & + & ++ & \text { größenabh. } \\ \text { Größe } & +++ & + & +++ & + \\ \text { DC-Betrieb } & \text { ja } & \text { ja } & \text { ja } & \text { nein } \\ \text { Kosten } & +++ & + & +++ & \end{array}$

Abbildung 8.15: Tabellarischer Vergleich der aktuellen Sensormaterialien; aus [117]

Ein Spezialfall der Sensorik sind die Leseköpfe in Festplatten. Die ersten kommerziell erhältlichen Festplatten sind im Jahr 1956 von IBM für einen Preis von 10.000 US\$ vorgestellt worden. Sie besitzt eine Gesamtspeicherkapazität von $5 \mathrm{MB}$, die auf 50 Platten verteilt vorliegt. Im direkten Vergleich zu den heutigen Festplattenspeichern zeigt sich die rasante Entwicklung bei diesen Medien: 


\begin{tabular}{|c|c|}
\hline Jahr & Speicherkapazität pro Scheibe \\
\hline 1956 & $1 \mathrm{MB}$ \\
\hline 2003 & $80 \mathrm{~GB}^{46}(80.000 \mathrm{MB})$ \\
\hline
\end{tabular}

Die immer stärker wachsende Datenmenge im Alltag fordert eine fortschreitende Miniaturisierung und eine höhere Speicherdichte, die bei kleinen Produkten mit herkömmlichen Sensoren nicht mehr zu bewerkstelligen sind. Anfang der neunziger Jahre wurde der erste $\mathrm{AMR}^{47}$ - Festplattenkopf eingeführt, was zu einer deutlichen Zunahme der Speicherdichte führte (Abbildung 8.16 zeigt das exponentielle Wachstum seit Einführung der MR - Sensoren). Nur wenige Jahre später (1997) wurden diese von den GMR - Sensoren abgelöst, wodurch die Datendichte weiter erhöhet werden konnte. GMR - Sensoren besitzen einen im Vergleich zu AMR - Sensoren höheren Magnetowiderstandseffekt (AMR: $2 \%<->$ GMR: 20-50 \% [118]), wodurch weiterführende, dimensionsbezogene Reduktionen möglich sind.

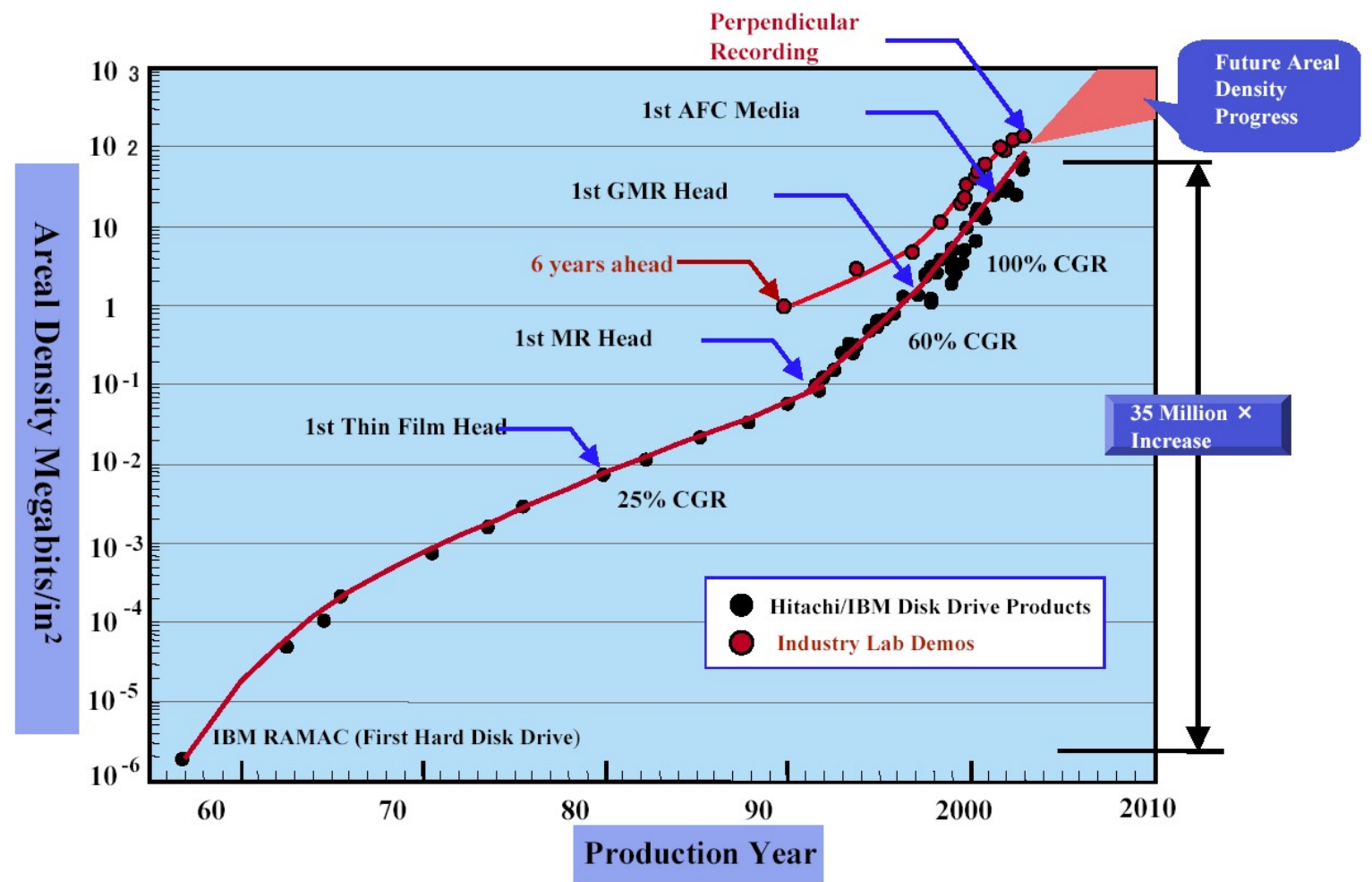

Abbildung 8.16: Entwicklung der Speicherdichte seit 1956 (aus [119] )

Aber auch der Miniaturisierung von GMR - Sensoren sind Grenzen gesetzt: Verkleinerungen der lateralen Dimension können bei diesen Schichtsystemen zu geringeren Widerstanden führen und somit die Einsatzfähigkeit aufgrund der verminderten Detektierbarkeit von Signalen als Sensor

\footnotetext{
${ }^{46}$ Hitachi SATA - HDS722580VLAT20 (2003)

${ }^{47}$ Anisotropic MagnetoResistance
} 
verhindern. Abhilfe können hier $\mathrm{TMR}^{48}$ - Sensoren schaffen, die aufgrund einer dünnen isolierenden Schicht einen höheren Widerstand aufweisen. Da der Widerstand des TMR Elements exponentiell von der Schichtdicke des Isolators abhängt, ist es schwierig, einen genormten Herstellungsprozess zu finden, der eine Reproduzierbarkeit der Sensoren garantiert. Weiterführende Forschungs - und Entwicklungsarbeit ist für eine Massenproduktion nötig.

Ein Vergleich des weltweiten Handelvolumens für nichtflüchtige Speichermedien aus den Jahren 1998 und 2002 zeigt ein starkes Wachstum für den gesamten Bereich. Innerhalb dieser recht kurzen Zeitspanne ist der Umsatz weltweit von 74 Mrd. US-\$ auf 114 Mrd. US-\$ gestiegen - dies entspricht einem Wachstum von 54\%. Die prozentual höchsten Verluste mussten Diskettenlaufwerke ${ }^{49}$ und Bandlaufwerke verzeichnen, sie haben innerhalb dieser Zeit ein Drittel ihres Marktanteils verloren. Optische Datenspeicher (CD; DVD und MO) und Festplattenspeicher konnten die bisherige Marktposition verteidigen, wobei Festplattenspeicher trotz einer geringen Abnahme mit $33 \%$ im Jahr 2002 weiterhin in Führung liegen.

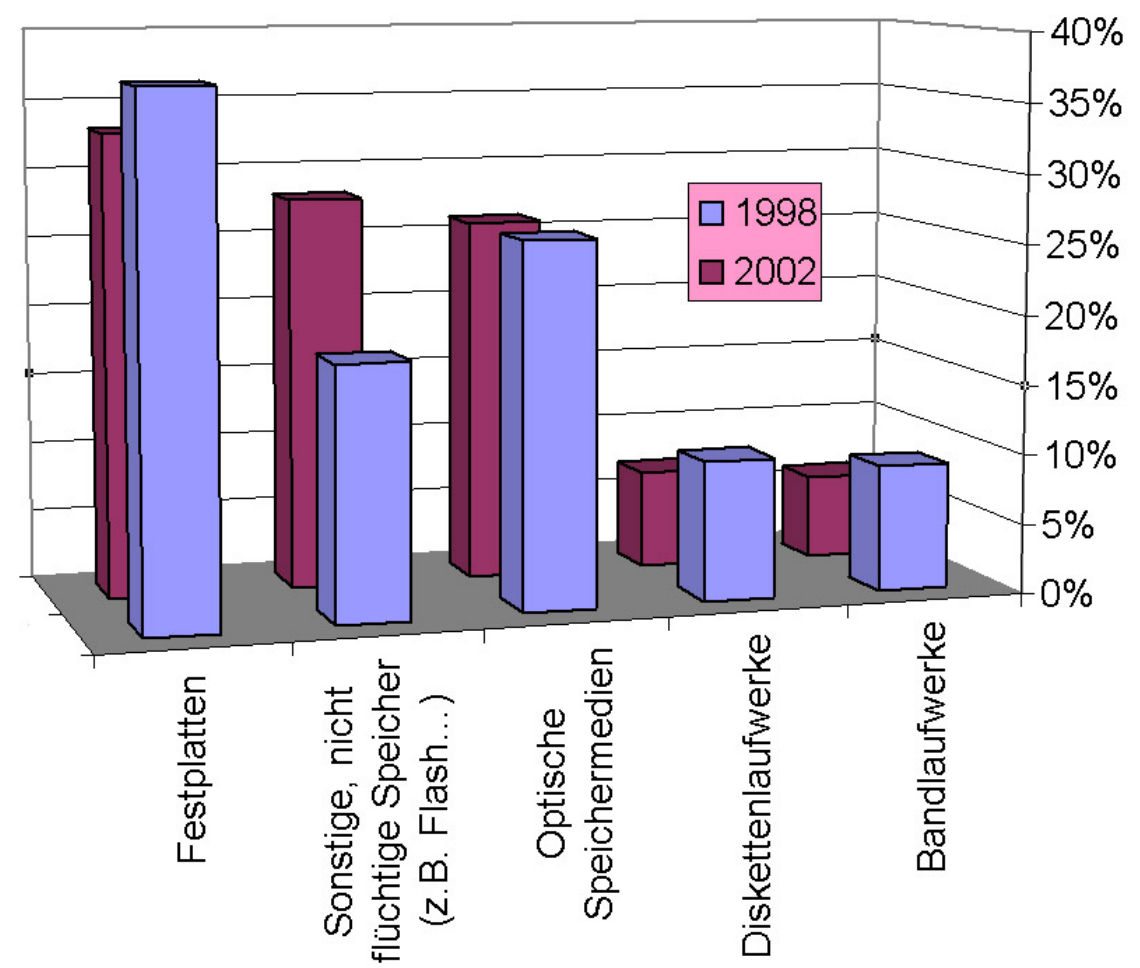

Abbildung 8.17: Verteilung der verschiedenen, nichtflüchtigen Speichermedien für die Jahre 1998 und 2002 gemessen am jährlichen Gesamtumsatz (aus [120])

\footnotetext{
48 Tunnel MagnetoResistance

${ }^{49}$ Hierzu zählen neben der Floppy auch Zip - Laufwerke und vergleichbare Technologien
} 
Am stärksten konnten die neu entwickelten, nicht flüchtigen Speichersysteme ${ }^{50}$ an Gesamtumsatz zulegen. Sie finden besonders Anwendung in transportablen Unterhaltungsmedien entsprechend MP3 - Playern, PDA's, Handys und Digitalkameras - Geräten, bei denen es besonders auf ein geringes Gewicht und eine kompakte Bauweise ankommt. Um auch in diesem Marktsegment vertreten zu sein haben IBM / Hitachi eine spezielle Festplattenkonfiguration entwickelt, das sogenannte „Microdrive“. Es besitzt den Formfaktor der CompactFlash - Module (CF) Typ II (ein Zoll Durchmesser) und Kapazitäten bis hin zu $4 \mathrm{~GB}^{51}$. CF - Module sind im Vergleich derzeit mit bis zu 4 GB erhältlich - Ende 2004 werden die ersten Medien mit bis zu 32 GB verfügbar sein $^{52}$. Ein zusätzlicher Vorteil für Microdrives sind die vergleichsweise niedrigeren Produktionskosten und somit niedrigeren Endverbraucherpreise. Als nachteilig sind bei diesen Medien jedoch ihre hohe Schockempfindlichkeit, ihr relativ hohes Gewicht und der erhöhte Energieverbrauch anzusehen.

Aufgrund der weiterführenden Miniaturisierung von Unterhaltungsmedien und einem stark applikationsgetriebenem Bedarf an hohen Speicherkapazitäten ist mit einem weiter ansteigenden Markt in diesem Segment zu rechnen. Führ das Jahr 2005 wird ein weltweiter Gesamtumsatz von $45 \mathrm{Mrd}$. US-\$ für diese Form von Speichersystemen prognostiziert [121].

Die weitaus kürzere Lebenszeit ${ }^{53}$ und höheren Schreibzeiten [122] sprechen aber auf Dauer gegen die Flash - Technologie und treiben die Entwicklung neuer Technologien an. Aktuelle Speicher, die auf $\mathrm{DRAM}^{54}$ - Technik basieren, besitzen zwar eine niedrige Zugriffszeit (ns) und eine vergleichsweise hohe Lebenserwartung - sie verlieren aber bei Abschaltung der elektrischen Versorgung die in ihnen gespeicherten digitalen Informationen und sind somit für die oben genannten Einsatzzwecke ungeeignet. Als eine Kombination der Vorteile beider Systeme werden im Augenblick die $\mathrm{MRAM}^{55}$ - und die $\mathrm{RRAM}^{56}$ - Technologie gehandelt. Beide Konzepte besitzen im Vergleich zum DRAM den Vorteil, dass sie Daten „nicht - flüchtig“ speichern können. Sie besitzen somit die Fähigkeit, gleichzeitig als Arbeitsspeicher und als Festspeicher zu fungieren, was zu kleineren Abmessungen und günstigeren Geräten führen kann. Sie zeichnen sich weiter durch einen geringeren Stromverbrauch, erhöhte Transportabilität und Flexibilität aus. Die MRAM - Technologie basiert auf dem Prinzip der bis in die 70er Jahre verwandten Ringkernspeichertechnik: Informationen werden als „0“ oder „1“ gespeichert, indem die

\footnotetext{
${ }^{50}$ Diese Rubrik umfasst alle neuen Speichersysteme, die als Festplattenspeicherersatz in Betracht kommen. Am wohl bekanntesten sind die Vertreter der Flash - Technologie.

51 Stand: Ende 2003

52 Herstellerangabe: Hitachi Inc.

${ }^{53}$ maximale Lebensdauer von 100.000 Schreibzyklen

${ }^{54}$ Dynamic Random Access Memory (engl.): Speicher mit wahlfreiem Zugriff, d.h. lesbarer und beschreibbarer Speicher

${ }^{55}$ Magnetic Random Access Memory

56 magnetoResistive Random Access Memory
} 
Hysterese von ferromagnetischen Materialien ausgenutzt wird. Erste Konzepte zum MRAM und ein Prototyp eines $256 \mathrm{~K}$ MRAM Chips (Abbildung 8.18) wurden in den 80er Jahren von der Firma Honeywell vorgestellt.

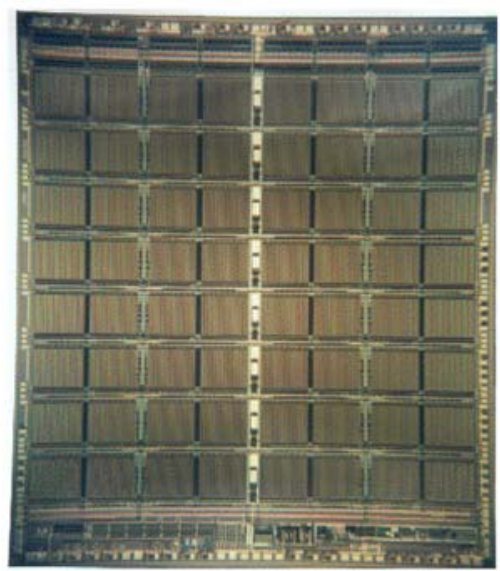

Abbildung 8.18: MRAM - Prototyp: 256 K Chip der Firma Honeywell Inc. [123]

Leider besaßen die damaligen Materialien nicht die gewünschten Eigenschaften, so dass der Signalunterschied im Bereich von wenigen Prozenten lag. Erst die Entdeckung des GMR Effektes Anfang der 90er Jahre gab wieder Anlass zur Hoffnung: GMR - Systeme zeigten einen MR - Effekt von 6\%. Allerdings konnte aufgrund des starken Pinnings der Magnetisierung entlang der Zellen und des immer noch recht schwachen Signalunterschiedes eine minimale Linienbreite von $1 \mu \mathrm{m}$ nicht unterschritten werden - eine stärkere Miniaturisierung blieb dem System somit verwehrt. Die Einführung magnetischer Tunnelkontakte ${ }^{57}$ behob diese Begrenzung. TMR - Werte von 40\% und mehr boten eine gute Ausgangslage für eine weitere Entwicklung.
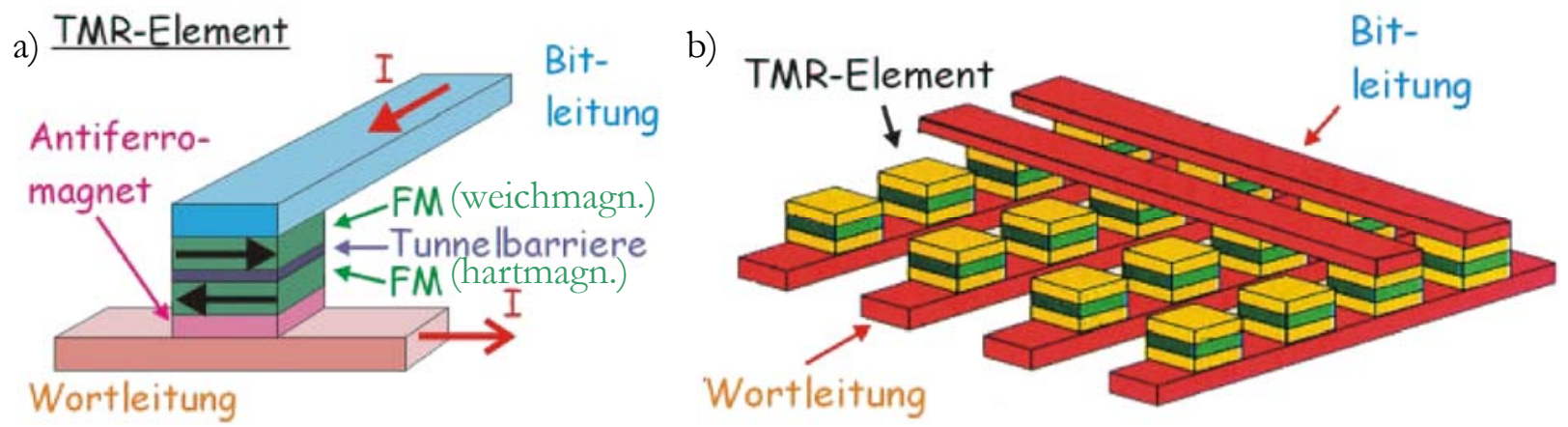

Abbildung 8.19: Schematischer Aufbau einer MRAM - Speicherzelle (aus[124] )

a) Einzelne TMR - Speicherzelle

b) Array aus $4 \times 4$ TMR - Elementen

\footnotetext{
${ }^{57}$ MTJ: Magnetic Tunnel Junction
} 
In Abbildung 8.19 ist ein MRAM - Speicher in cross - point - array - Architektur basierend auf dem TMR - Effekt skizziert. Die einzelne Speicherzelle besteht prinzipiell aus drei Lagen: zwei magnetischen Schichten, zwischen denen sich eine nichtmagnetische Schicht befindet, wobei jede ca. 3 bis 6 Nanometer dünn ist. Die obere Schicht ist weichmagnetisch, wodurch kleine Ströme in der Lage sind, das Magnetfeld in seiner Richtung zu verändern. Die untere Schicht besitzt als Referenz eine feste Magnetfeldrichtung - sie ist hartmagnetisch. Der Magnetisierungs Unterschied zwischen den beiden Schichten dient zur Unterscheidung des Speicherzelleninhaltes. Die Bit - bzw. Word - Line dienen zum Schreiben und zum Auslesen der Information $^{58}$.

Infineon und IBM kooperieren seit ca. 10 Jahren im Bereich der MRAM - Technologien und ergänzen sich aufgrund von Kostenersparnissen und der Zusammenführung von Kompetenzen: IBM hält die meistzitierten MRAM - Patente, Infineon besitzt außerordentliche Erfahrungen in der Produktion hochdichter Speicherbausteine und forscht ebenfalls aktiv an MRAM. Als ein Ergebnis dieser Kooperation ist auf dem VLSI - Symposium in Kyoto (6/2003) der erste in $0,18 \mu \mathrm{m}$ gefertigte $128 \mathrm{~KB}$ MRAM Core, basierend auf dem TMR-Effekt, der Weltöffentlichkeit vorgestellt worden. Sie haben somit gezeigt, dass es möglich ist, sehr kleine MRAM - Speicherzellen mit einer Fläche von nur 1,4 $\mu \mathrm{m}^{2} \mathrm{zu}$ erzeugen.
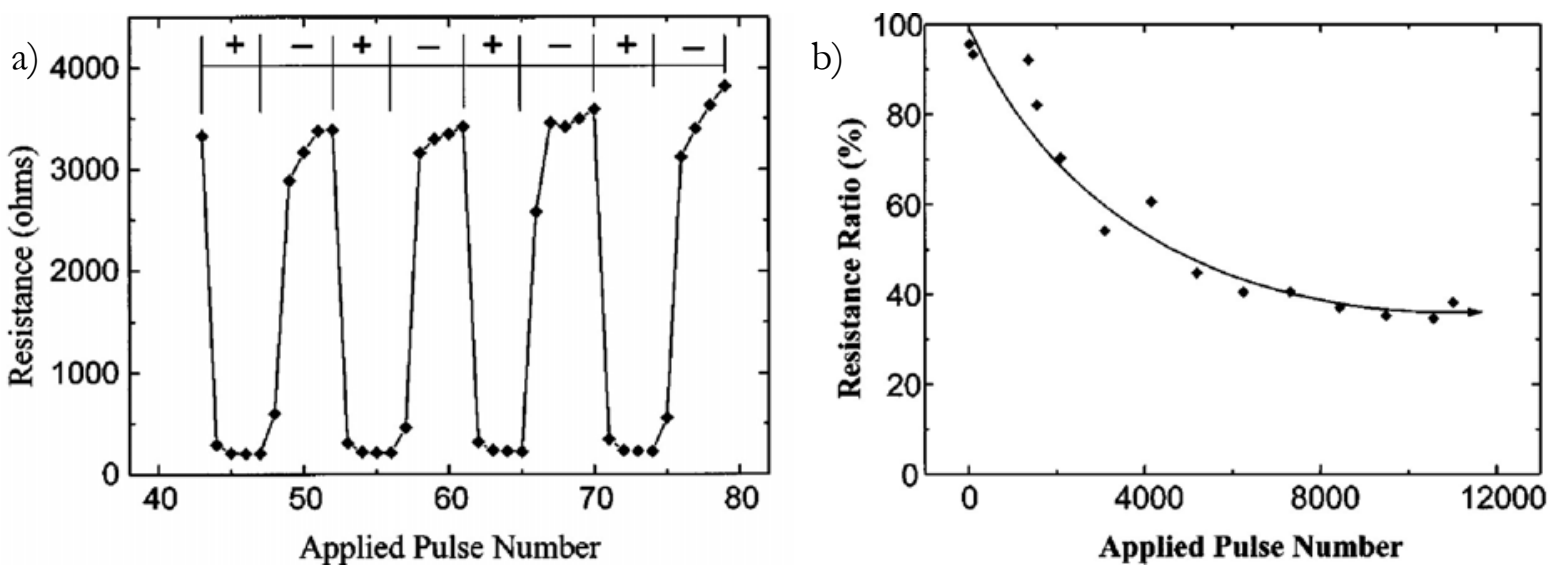

Abbildung 8.20: a) Widerstandsänderung eines PCMO - Filmes durch kurzzeitige Pulse ( 100 ns) von \pm 5 bei Raumtemperatur und ohne magnetisches Feld

b) Reproduzierbarkeit der Widerstandsänderung nach einer hohen Anzahl von Pulsen

Als eine Alternative zum MRAM - Konzept ist der RRAM zu sehen. Dementsprechend sieht die Industrie [125] ein hohes Entwicklungspotential für diese Materialien als zukünftige

\footnotetext{
58 Weiterführende Informationen zur technologischen Umsetzung und zum Funktionsprinzip sind in [126] und [127] zu finden.
} 
Speichermedien. Überlegungen zur Herstellung von RRAM's belegen dies [128]. Manganate sollen hierbei als Träger der Information eingesetzt werden und als Speicherzelle fungieren. Aufgrund des großen CMR - Effektes besteht die Möglichkeit, die einzelnen Zellen im Vergleich zu den TMR - basierten Elementen weiter zu verkleinern. Erste Ergebnisse zum Schaltverhalten durch elektrische Felder sind recht jung und wurden 1997 veröffentlicht [129]. Es ist gezeigt worden, dass $\mathrm{Pr}_{0.7} \mathrm{Ca}_{0.3} \mathrm{MnO}_{3}$ (PCMO) bei einer angelegten Schaltspannung von $700 \mathrm{~V}$ reproduzierbar eine Widerstandsänderung aufweist. Dass ein Umschaltprozess auch bei weit aus niedrigeren Spannungen an PCMO ermöglicht werden kann, ist in [130] vorgestellt worden. Um Widerstandsänderungen hervorzurufen wurden hier $\pm 5 \mathrm{~V}$ Pulse mit einer Durchschnittsdauer von ca. 100ns angelegt (Abbildung 8.20 (a)) und gezeigt, dass dies auch nach einer recht hohen Anzahl von Pulsen noch möglich ist (siehe (b)). Die Widerstandsänderung hat sich nach ca. 10.000 „Schreibzyklen“ auf einen Wert von 40 \% eingestellt.

Somit zeigt diese Materialklasse gute Eigenschaften, um als mögliches Speichermedium eingesetzt zu werden. Es bleibt die abschließende Frage, ob auch hier Grenzen bei der Miniaturisierung der einzelnen Zellen existieren bzw. ob diese Materialien ihre Eigenschaften zu verkleinerten Systemen hin ändern. Entsprechend den vorgestellten Ergebnissen kann eine bestehende Phasenseparation hierauf Einfluss nehmen und ggf. als begrenzendes Kriterium angesehen werden. 


\section{Literaturverzeichnis}

1 - R. von Helmholt, J. Wecker, B. Holzapfel, L. Schulz, und K. Samwer, giant negative magnetoresistance in perovskitelike $\mathrm{La}_{2 / 3} \mathrm{Ba}_{1 / 3} \mathrm{MnO}_{3}$ ferromagnetic films, Phys. Rev. Lett. 71, 2331 (1993); R. von Helmholt, J. Wecker, K. Samwer, L. Haupt und K. Bärner, intrinsic giant magnetoresistance of mixed valence La-A-Mn oxide (a=Ca, Sr, Ba), J. Appl. Phys. 76, 6925 (1994)

2 - H. A. Jahn und E. Teller,Stability of polyatomic molecules in degenerate electronic states, I. orbital degeneracy, Proc. Roy. Soc., London 161, 220 (1937)

3 - A.J. Millis, lattice effects in magnetoresistive manganese perovskites, Nature 392, 147 (1998)

4 - J. B. Googenough, theory of the role of covalence in perovskite-type manganites [La,M(II)] $\mathrm{MnO}_{3}$, Phys. Rev. 100, 564 (1955)

5 - S. Yunoki, A. Moreo, E. Dagotto, phase separation induced by orbital degrees of freedom in models for manganites with Jahn - Teller phonons, Phys. Rev. Lett. 81, 5612 (1998)

6 - M. Mayr, A. Moreo, J. A. Vergès, J. Arispe, A. Feiguin und E. Dagotto, resistivity of mixed phase manganites, Phys. Rev. Lett. 86, 135 (2001)

7 - J. Burgy, M. Mayr, V. Martin-Mayor, A. Moreo und E. Dagotto, colossal effects in transition metal oxides caused by intrinsic inhomogeneities, Phys. Rev. Lett. 87, 277202-1 (2001)

8 - A. Moreo, M. Mayr, A. Feiguin, S. Yunki und E. Dagotto, giant cluster coexistence in doped manganites aun other compounds, Phys. Rev. Lett. 84, 5568 (2000)

9 - S. Kirkpatrick, percolation und conduction, Rev. Mod. Phys. 45, 574 (1973)

10 - M. Fäth, S. Freisem, A. A. Menovsky, Y. Tomioka, J. aarts und J. A. Mydosh, spatially inhomogenous metal-insulator transition in doped manganites, Science 285, 1540 (1999) 
11 - C. Renner, G. Aeppli, B.-G. Kim, Y.-A. Soh und S.-W. Cheong, atomic-scale images of charge ordering in a mixed-valence manganite, Nature 416, 518 (2002)

12 - V. M. Goldschmidt, Skrifter Norske Videnskaps-Akademie Oslo, I. Mat. Nat. Kl., 8, (1926)

13 - J.B. Goodenough und J. M. Longo, Landholt-Brönstein, Tabellen III 4a, 6. Auflage, Springer Verlag, Berlin,132 (1970)

14 - J. M. D. Coey, M. Viret, S. von Molnar, Mixed-valence manganites, Adv. Phys. 48, 167 (1999)

15 - Ch. E. Mortimer, Chemie, 5. Auflage, Georg Thieme Verlag Stuttgart, 484 (1987)

16 - W. Zinn, Elektronen in Oxiden, Jahn-Teller-Effekt, Jülich - Ferienkurs, Supraleitung und verwandte Quantenphänomene, 1988

17 - F. Moussa, M. Hennion, J. Rodriguez-Carvajal, H. Moudden, L. Pinsard und A. Revcolevschi, spin waves in the antiferromagnet perovskite $\mathrm{LaMnO}_{3}$ : a neutron-scattering study, Phys. Rev. B 54, 15149 (1996)

18 - E. O. Wollan und W. C. Koehler, neutron diffraction study of the magnetic properties of the series of perovskite-type compounds [(1-x)La,xCa] $\mathrm{MnO}_{3}$, Phys. Rev. 100, 545 (1955)

19 - W. E. Pickett und D. J. Singh, electronic structure and half-metallic transport un the $\mathrm{La}_{1-}$ ${ }_{\mathrm{x}} \mathrm{Ca}_{\mathrm{x}} \mathrm{MnO}_{3}$, Phys. Rev. B 53, 1146 (1996)

20 - P.-G. de Gennes, effects of double excange in magnetic crystals, Phys. Rev. 118, 141 (1960)

21 - S. Satpathy, Z. S. Popovic und F. R. Vukajlovic, electronic structure of the perovskite oxides: $\mathrm{La}_{1-\mathrm{x}} \mathrm{Ca}_{\mathrm{x}} \mathrm{MnO}_{3}$, Phys. Rev. Lett. 76, 960 (1996)

22 - P. Mahadevan, K. Terakura und D. D. Sarma, spin, charge and orbital ordering in $\mathrm{La}_{0.5} \mathrm{Sr}_{0.5} \mathrm{MnO}_{4}$, Phys. Rev. Lett. 87, 066404-1 (2001) 
23 - T. Saitoh, A. E. Bocquet, T. Mizokawa, H. Namatame und A. Fujimori, electronic structure of $\mathrm{La}_{1-\mathrm{x}} \mathrm{Sr}_{\mathrm{x}} \mathrm{MnO}_{3}$ studied by photoemission and x-ray-absorption spectroscopy, Phys. Rev. B 51, $13942(1995)$

24 - A. Chainani, M. Mathew und D. D. Sarma, electron spectroscopy investigation of the semiconducter-metal transition in $\mathrm{La}_{1-\mathrm{x}} \mathrm{Ca}_{\mathrm{x}} \mathrm{MnO}_{3}$, Phys. Rev. B 47, 15397 (1993)

25 - M. Abbate, F. M. F. de Groot, J. C. Fuggle, A. Fujimori, O. Strebel, F. Lopez, M. Domke, G. Kaindl, G. A. Sawatzky, M. Takano, Y. Takeda, H. Eisaki und S. Uchida, controlled-valence properties of $\mathrm{La}_{1-\mathrm{x}} \mathrm{Sr}_{\mathrm{x}} \mathrm{FeO}_{3}$ and $\mathrm{La}_{1-\mathrm{x}} \mathrm{Sr}_{\mathrm{x}} \mathrm{MnO}_{3}$ studied by soft-x-ray absorption spectroscopy, Phys. Rev. B 46, 4511 (1992)

26 - P. Schiffer, A. P. Ramirez, W. Bao und S.-W. Cheong, low temperature magnetoresistance and the magnetic phase diagram of $\mathrm{La}_{1-\mathrm{x}} \mathrm{Ca}_{\mathrm{x}} \mathrm{MnO}_{3}$, Phys. Rev. Lett. 75, 3336 (1995)

27 - A. Moreo, S. Yunoki und E. Dagotto, phase separation scenario for manganese oxides and related materials, Science 283, 2034 (1999)

28 - A. Urushibara, Y. Moritomo, T. Arima, A. Asamitsu, G. Kido und Y. Tokura, isulator-metal transition and giant magnetorisistance in $\mathrm{La}_{1-\mathrm{x}} \mathrm{Sr}_{\mathrm{x}} \mathrm{MnO}_{3}$, Phys. Rev B 51, 14103 (1995)

29 - M. Paraskevopoulos, F. Mayr, J. Hemberger, A. Loidl, R. Heichele, D. Maurer, V. Müller, A. A. Mukhin und A. M. Balbashov, magnetic properties and the phase diagram of $\mathrm{La}_{1-\mathrm{x}} \mathrm{Sr}_{\mathrm{x}} \mathrm{MnO}_{3}$ for x $\leq$ 0.20, J. Magn. Magn. Mater. 211, 118-27 (2000)

30 - T. Kurz, Charakterisierung und Phasendiagramm von $\mathrm{La}_{1-\mathrm{x}} \mathrm{Sr}_{\mathrm{x}} \mathrm{MnO}_{3}$, Diplomarbeit - Institut für Physik - Universität Augsburg (2001)

31 - K. Samwer, Kolossaler Magnetowiderstand (CMR) in Manganaten - ein Metall-Isolator Übergang im äußeren Magnetfeld, Jülich - Ferienkurs, D9, 1999

32 - W. Nolting, Quantentheorie des Magnetismus, 5.3.2. Superaustausch, Teubner, 271, (1986)

33 - J. H. van Santen und G. H. Jonker, electrical conductivity of ferromagnetic compounds of manganese with perovskite structure, Physica XVI, 599 (1950) 
34 - G. H. Jonker, magnetic compounds with perovskite structure - conducting and nonconducting compounds, Physica XXII, 707 (1956)

35 - G. H. Jonker, semiconducting properties of mixed crystals with perovskite structure, Physica XX, 1118 (1956)

36 - Zener, interaction between the d shells in transition metals - II. ferromagnetic compounds of manganese with perovskite struktur, Phys. Rev. 82, 403 (1951)

37 - E. O. Wollan und W. C. Köhler, neutron diffraction study of the magnetic properties of the series of perovskite-type compounds [(1-x)La,xCA] $\mathrm{MnO}_{3}$, Phys. Rev. 100 ,2 (1955)

38 - P. W. Anderson und H. Hasegawa, considerations on double exchange, Phys. Rev. 100, 675 (1955)

39 - S. Köster, Änderung der Magnetowiderstandseffekte in $\mathrm{La}_{0.7} \mathrm{Sr}_{0.3} \mathrm{MnO}_{3}-$ Filmen durch Dotierung der Korngrenzen mit MgO, Diplomarbeit - I. Physikalisches Institut - Universität Göttingen, $120(2002)$

40 - F. Müller, Resistive und magnetische Eigenschaften von Manganat-Multilagen, Diplomarbeit I. Physikalisches Institut - Universität Göttingen, 78 (2001)

41 - W. Nolting, Quantentheorie des Magnetismus, 5.3.3. Doppelaustausch, Teubner, 280, (1986)

42 - A. J. Millis, P.B. Littlewood und B. I. Shraiman, double exchange alone does not explain the resistivity of $\mathrm{La}_{1-\mathrm{x}} \mathrm{Sr}_{\mathbf{x}} \mathrm{MnO}_{3}$, Phys. Rev. Lett. 74, 5144 (1995)

43 - A. J. Millis, B. I. Shraiman und R. Mueller, dynamic Jahn-Teller Effect and colossal magnetoresistance in $\mathrm{La}_{1-\mathrm{x}} \mathrm{Sr}_{x} \mathrm{MnO}_{3}$, Phys. Rev. B 77, 175 (1996)

44 - E. Dagotto, nanoscale phase separation and colossal magnetoresistance - the physics of manganites and related compounds, Springer Verlag, (2003)

45 - S. Yunoki, J. Hu, A. L. Malvezzi, A. Moreo, N. Furukawa und E. Dagotto, phase separation in electronic models for manganites, Phys. Rev. Lett. 80, 845 (1998) 
46 - K. A. Müller und G. Benedek, proc. of the conference phase separation in cuprate superconductors, world scientific, Singapore (1993)

47 - E. L. Nagaev, ground state anomalous magnetic moment of conduction electrons in an antiferromagnetic semiconductor, JETP Lett. 6, 16 (1967) (Original: ZhETF Pis'ma 6, No. 1, 484 (1967)

48 - N. Mathur und P. Littlewood, mesoscopic texture in manganites, Physics Today 1, 25 (2003)

49 - R.M. Kusters, J. Singleton, D.A. Keen, R. McGreevy und W. Hayes, magnetoresistance measurements on the magnetic semiconductor $\mathrm{Nd}_{0.5} \mathrm{~Pb}_{0.5} \mathrm{MnO}_{3}$, Physica B 155, 362 (1989)

50 - M. Uehara, S. Mori, C. H. Chen und S.-W. Cheong, percolative phase separation underlines colossal magnetoresistance in mixed-valent manganites, nature 399, 560 (1999)

51 - http://www.agnld.uni-potsdam.de/ shw/TSA/Tools/ClusterAnaStefan/node8.html

52 - G. Binnig und H. Rohrer, scanning tunneling microscopy, Helv. Phys. Acta 55, 726-735 (1982)

53 - G. Binning, H. Rohrer, Ch. Gerber und E. Weibel, surface studies by scanning tunnelling microscopy, Phys. Rev. Lett. 49, 57 (1982)

54 - G. Binning und H. Rohrer, scanning tunnelling microscopy - from birth to adolescence, Rev. Mod. Phys. 59, 615 (1987)

55 - K. Sauthoff, Rastertunnelspektroskopie an einzelnen Donatoren und Donatorkomplexen nahe der GaAs(110) - Oberfläche, Dissertation an der Georg-August-Universität ₹u Göttingen (2003)

56 - T. Reusch, cross-sectional scanning tunneling microscopy of Au contacts on GaAs(110), Dissertation an der Georg-August-Universität ₹u Göttingen (2003)

57 - Omicron VT-STM Betriebsanleitung, Version 1.5, 84 (1998)

58 - Preparation and characterization of electrochemically etched W Tips for STM, Meas. Sci. Technol. 10, 11 (1999) 
$59-\mathrm{J}$. Tersoff und D. R. Hamann, theory of the scanning tunnelling microscope, Phys. Rev. B 31, 805 (1985)

60 - R. Hamers, R. Tromp und J. Demuth, electronic and geometric structure of $\operatorname{Si}(111)-(7 \times 7)$ and $\mathrm{Si}(001)$ surfaces, Surface Sci. 181, 346 (1987)

61 - J. Bardeen, tunnelling from a many-particle point of view, Phys. Rev. Lett. 6, 57 (1961)

62 - S. Blügel, Theorie der Rastertunnelmikroskopie, 29. Jülich - Ferienkurs, A3

63 - Omicron Scala Softwarehandbuch, Version 4.0, 77 (2001)

64 - V. Moshnyaga, I. Khoroshun, A. Sidorenko, P. Petrenoko, A. Wiedinger, M. Zeitler, B. Rauschenbach, R. Tidecks und K. Samwer, preparation of rare-earth manganite-oxide thin films by metalorganic aerosol deposition technique, Appl. Phys. Lett. 74, 2842 (1999)

65 - A. Biswas, A. K. Raychaudhuri, R. Mahendiran, A. Guha, R. Mahesh und C. N. R. Rao, direct measurement of the charge ordering gap in $\mathrm{Nd}_{0.5} \mathrm{Sr}_{0.5} \mathrm{MnO}_{3}$ using vacuum tunnelling, $J$. Phys: Condens. Matter 9, L355 (1997)

66 - A. Biswas, S. Elizabeth, A. K. Raychaudhuri and H. L. Bhat, density of states of hole-doped manganites: a scanning-tunneling-microscopy / spectroscopy study, Phys. Rev. B 59, 5368 (1999)

67 - R. Stratton, volt - current characteristics for tunneling through insulating films, J. Phys. Chem. Solids 23, 1177 (1962)

68 - C. Hamann und M. Hietschold, Raster-Tunnel-Mikroskopie, Akademie - Verlag Berlin S. 19 (1991)

69 - http://www.goodfellow.com/csp/active/static/G/AU00.HTML

70 - T. Habermann, Rastermikroskopische Untersuchungen der Feldemission von Metall- und Diamantkathoden, Dissertation an der Universität ₹u Wuppertal (1999) 
71 - V. Moshnyaga, I. Khoroshun, A. Wiedinger, S. Klimm, M. Zeitler, R. Tidecks, S. Horn und K. Samwer, magnetotransport in chemically derived La-Ca-Mn-O thin films, J. Appl. Phys. 86, $5642(1999)$

72 - U. Geyer, Rastertunnelmikroskopie an $\mathrm{YBa}_{2} \mathrm{Cu}_{3} \mathrm{O}_{7}$ - Schichten: Interpretation der Bilder und der spektroskopischen Ergebnisse, Dissertation an der Georg-August-Universität zu Göttingen (1992)

73 - Y. Luo, A. Käufler und K. Samwer, magnetotunneling in sputtered $\mathrm{La}_{0.7} \mathrm{Ca}_{0.3} \mathrm{MnO}_{3} / \mathrm{Al}_{2} \mathrm{O}_{3}$ multilayers, Appl. Phys. Lett. 77, 1508 (2000)

$74-$ Y. Luo und K. Samwer, structural and magnetotunneling investigations on $\mathrm{La}_{0.7} \mathrm{Ca}_{0.3} \mathrm{MnO}_{3} /$ $\mathrm{Al}_{2} \mathrm{O}_{3}$ multilayers, J. Appl. Phys. 86, 6760 (2001)

75 - T. Becker, C. Streng, Y. Luo, V. Moshnyaga, B. Damaschke, N. Shannon und K. Samwer, intrinsic inhomogeneities in manganite thin films investigated with scanning tunneling spectroscopy, Phys. Rev. Lett. 89, 237203-1 (2002)

76 - A. Gieske, Eigenschaften von dünnen epitaktischen Manganatschichten in frühen Wachstumsstadien, Diplomarbeit - I. Physikalisches Institut - Universität Göttingen, (2003)

77 - Y. Luo persönliche Mitteilung

78 - J. G. Simmons und R. R. Verderber, new conduction and reversible memory phenomena in thin insulating films, proc. Roy. Soc. A. 301, 77 (1967)

79 - C. Kittel, Einführung in die Festkörperphysik, Oldenbourg Verlag München (1996)

80 - J. M. De Teresa, M. R. Ibarra, P. A. Algarabel, C. Ritter, C. Marquina, J. Blasco, J. Garcia, A. del Moral und Z. Arnold, evidence for magnetic polarons in the magnetoresistive perovskites, Nature 386, 256 (1997)

81 - N. W. Ashcroft und N. D. Mermin, solid state physics, Saunders College Publishing 564 (1976) 
82 - M. R. Ibarra und J. M. De Teresa, colossal magnetoresistance in manganese oxide perovskites, Magn. Magn. Mater. 177-181, 849 (1998)

83 - K. H. Ahn, T. Lookmann und A. R. Bishop, strain - induced metal-insulator phase coexistence in perovskite manganites, Nature 428, 401 (2004)

84 - J. C. Loudon, N. D. Mathur und P. A. Midgley, charge - ordered ferromagnetic phase in $\mathrm{La}_{0.5} \mathrm{Ca}_{0.5} \mathrm{MnO}_{3}$, Nature 420, 797 (2002)

85 - C. Streng, Wachstumsanalyse amorpher dicker Schichten und Schichtsysteme, Dissertation an der Georg-August-Universität ₹u Göttingen (2004)

86 - W. Bao, J.D. Axe, C.H. Chen und S.-W. Cheong, impact of charge ordering on magnetic correlations in perovskite (Bi, Ca) $\mathrm{MnO}_{3}$, Phys. Rev. Lett. 78, 543 (1997)

87 - G. Allodi, R. De Renzi, G. Guidi, F. Licci und M. W. Pieper, electronic phase separation in lanthanum manganites: evidence from ${ }^{55} \mathrm{Mn}$ NMR, Phys. Rev. B 56, 6036 (1997)

88 - M. Hennion, F. Moussa, G. Biotteau, J. Rodriguez-Carvajal, L. Pinsard und A. Revcolevschi, liquidlike spatial distribution of magnetic droplets revealed by neutron scattering in $\mathrm{La}_{1-\mathrm{x}} \mathrm{Ca}_{\mathrm{x}} \mathrm{O}_{3}$, Phys. Rev. Lett. 81, 1957 (1998)

89 - J. Fan, L. Pi, W. Tong, S. Xu, J. Gao, C. Zha und Y. Zhang, percolative conductivity in the $\mathrm{La}_{0.67} \mathrm{Sr}_{0.33} \mathrm{Mn}_{1-\mathrm{x}} \mathrm{Mg}_{\mathrm{x}} \mathrm{O}_{3}$ system, Phys. Rev. B 68, 092407-1 (2003)

90 - D. Niebieskikwiat, R. D. Sanchez, L. Morales und B. Maiorov, hole-doping dependence of perkolative phase separation in $\mathrm{Pr}_{0.5-\delta} \mathrm{Ca}_{0.2+\delta} \mathrm{Sr}_{0.3} \mathrm{MnO}_{3}$ around half doping, Phys. Rev. $B$ 66, 134422-1 (2002)

91 - J. Burgy, E. Dagotto und M. Mayr, percolative transitions with first-order characteristics in the context of colossal magnetoresistance manganites, Phys. Rev. B 67, 014410-1 (2003)

92 - J. Burgy, numerical studies of manganites models, Dissertation - Florida State University Departement of Physics (2003) 
93 - D. I. Khomskii und K. I. Kugel, elastic interaction and superstructures in manganites and other Jahn - Teller systems, cond-mat 0112340v1 (2001) (unveröffentlicht)

94 - G. Y. Vichniac, simulating physics with cellular automata, Physica D, S.96 (1984)

Eine Zusammenfassung mit Beispielen ist unter folgender Adresse $\mathrm{zu}$ finden: http://www.ifs.tuwien.ac.at/ aschatt/info/ca/ca.html

95 - D. Stauffer und A. Aharony, Perkolationstheorie - Eine Einfuhrung, VCH Verlagsgesellschaft $m b H,(1995)$

96 - Frederich Reif, Statistische Physik und Theorie der Wärme, WdeG Berlin 1987

97 - R. Skomski und P. A. Dowben, the finite - temperature densities ob states for half - metallic ferromagnets, Europhys. Lett. 58, 544 (2002)

98 - P. A. Dowben und R. Skomski, finite - temperature spin polarization in half-metallic ferromagnets, J. Appl. Phys. 93, 7948 (2003)

99 - F. Giessen, B. Damaschke, V. Moshnyaga, K. Samwer und G. A. Müller, suppression of interface - induced electrical phase separation in all - manganite multilayers by preservation of the Mn - O chain network, Phys. Rev. B 69, 014421 (2004)

100 - M. Bibes, L. Balcells, S. Valencia, J. Fontcuberta, M. Wojcik, E. Jedryka und S. Nadolski, nanoscale multiphase separation at $\mathrm{La}_{2 / 3} \mathrm{Ca}_{1 / 3} \mathrm{MnO}_{3} / \mathrm{SrTiO}_{3}$ Interfeces, Phys. Rev. Lett. 87, 067210-1 (2001)

101 - R. Akiyama, H. Tanaka, T. Matsumoto und T. Kawai, spin - polarized scanning tunneling microscopy on half - metallic manganite thin films with half - metallic manganite tip, Appl. Phys. Lett. 79, 4378 (2001)

102 - K. Dörr, J. M. De Teresa, K.-H. Müller, D. Eckert, T. Walter, E. Vlakhov, K. Nenkov und L. Schultz, preparation and properties of epitaxial $\mathrm{La}_{0.7} \mathrm{Ca}_{0.3} \mathrm{MnO}_{3-\delta}$ films with reduced carrier density, J. Phys.: Condens. Matter 12, 7099 (2000)

103 - Sir N. F. Mott, metal-insulator transitions, Taylor \& Francis Ltd. (1990) 
104 - M. Imada, A. Fujimori und Y. Tokura, metal-insulator transitions, Rev. Mod. Phys. 70, 1144 (1998)

105 - D. B. Mc Whan, A. Menth, J. P. Remeika, W. F. Brinkman und T. M. Rice, metal isolator transitions in pure and doped $\mathrm{V}_{2} \mathrm{O}_{3}$, Phys. Rev. B 7, 1920 (1973)

106 - D. B. Mc Whan, J. P. Remeika, T. M. Rice, W. F. Brinkman, J. P. Maita und A. Menth, electronic specific heat of metallic Ti - doped $\mathrm{V}_{2} \mathrm{O}_{3}$, Phys. Rev. Lett. 27, 941 (1971)

107 - C. Tusche, Magnetische Kopplung und Grenzflächenanisotropie von Eisen / Vanadiumsesquioxid- und Kobalt / Vanadiumsesquioxid - Doppellagen, Diplomarbeit - I. Physikalisches Institut - Universität Göttingen, (2003)

108 - B. Sass, C. Tusche, W. Felsch, N. Quaas, A. Weismann und M. Wenderoth, structural and electronic properties of epitaxial $\mathrm{V}_{2} \mathrm{O}_{3}$ thin films, J. Phys.: Condens. Matter 16, 77-87 (2004)

109 - W. Brückner, H. Oppermann, W. Reichelt, J. I. Terukow, F. A. Tschudnowski und E. Wolf, Vanadiumoxide, Akademie-Verlag Berlin (1983)

110 - K. Okazaki, A. Fujimori und M. Onoda, temperature-dependent electronic structure of $\mathrm{VO}_{2}$ in the insulating phase, J. Phys. Soc. Jap. 71, 822 (2002)

111 - M. Preisinger, J. Will, M. Klemm, S. Klimm und S. Horn, atomic scale imaging and spectroscopy of the $\mathrm{V}_{2} \mathrm{O}_{3}(0001)$ - surface: bulk versus surface effects, cond. Mat. $0111113 \mathrm{v} 1$ (2001)

112 - K. Bechgaard, K. Carneiro, M. Olsen, F. B. Rasmussen und C. S. Jacobsen, zero-pressure organic superconductor: Di-(Tetramethyltetraselenafulvalenium)-Perchlorate [(TMTSF) $\left.{ }_{2} \mathrm{ClO}_{4}\right]$, Phys. Rev. Lett. 46, 852 (1981)

113 - H. Uozaki, superconductivity, metal-insulator transition and magnetism in (BEDT$\mathrm{TSF})_{2} \mathrm{MCl}_{4}\left(\mathrm{M}=\mathrm{Fe}, \mathrm{Ga}, \mathrm{Ga}_{1-\mathrm{x}} \mathrm{Fe}_{\mathrm{x}}\right)$, Thesis, Physics Departement, Graduate School of Science, Tohoku University (March 2001) 
114 - T. Ishiguro, K. Yamaji, organic superconductors, Springer - Verlag, series in solid state science 88, (1990)

115 - M. N. Baibich, J.M. Broto, A. Fert, F. Nguyen Van Dau, F. Petroff, P. Eitenne, G. Creuzet, A. Friedrich und J. Chazelas, giant magnetoresistance of (001)Fe / (001) Cr magnetic superlattices, Phys. Rev. Lett. 61, 2472 (1988)

116 - Magnetismus / XMR-Technologien Technologieanalyse Magnetismus - xMRTechnologien Technologiefrüherkennung, Band 1: Molekulare Magnete, VDI-Technologiezentrum im Auftrag des Bundesministeriums für Bildung, Wissenschaft, Forschung und Technologie (BMBF), ISSN 1436-5928 (1999)

117 - HY-LINE Sensor-Tec Inselkammerstr. 10 D-82008 Unterhaching; www.hy-line.de

118 - WTEC Panel on spin electronics - Final Report - August 2003, WTEC Inc. 2809 Bosten St. Baltimore, MD 21224, 45 (2003)

119 - J. Naruse, HDD Business Strategy, towards the no. 1 supplier in the world, Hitachi Global Storage Technologies, www. Hitachi.com (2003)

120 - A. K. Menon und B. K. Gupta, data storage fort he millenium, datatech section 5: emerging trends, Henley Media Group - London

121 - R. M. Sherwin, memory on the move, IEEE Spectrum, 55 (2001)

122 - R. Sietmann, Das klassische DRAM bekommt Konkurrenz, c't - Magazin, Heise Verlag Hannover 6, 146 (1999)

123 - J. Daughton, magnetoresistive random access memory (MRAM), NVE Corporation, 11409 Valley View Road, USA, 4/2000

124 - J. König, U. Zülicke und G. Schön, Moderne Probleme der Spinelektronik und der Quanteninformationsverarbeitung, Institut für Theoretische Festkörperphysik - Universität Karlsrube, Vorlesung WS 2002/2003 
125 - S. Lai, Nonvolatile memory technologies: a look into the future, S. Lai, vice president, TMG, Intel Cooperation (2003)

126 - B. Dipert, exotic memories, diverse approaches, edn magazine (wnw.ednmag.com) 4, 57 (2001)

127 - R. Desikan, C. R. Lefurgy, S. W. Keckler, und D. Burger, on-chip as a high - bandwidth, low - latency replacement for DRAM physical memories, Department of Computer Science, Tech Report TR-02-47, University of Texas / Austin (2002)

128 - A. Ignatiev, X. M. Laio, Y. B. Nian, X. Chen, Z. J. Tang, C. Papagianni, N. J. Wu, J. R. Liu und W. K. Chu, fabrication and properties of electrical pulse induced resistive memory, Texas Center for Superconductivity and Advanced Materials - University of Houston, http://nvm.jpl.nasa.gov/Program.htm

129 - A. Asamitsu, Y. Tomioka, H. Kuwahara und Y. Tokura, current switching of resistive states in magnetoresisitve manganites, nature 388, 50 (1997)

130 - S. Q. Liu, N. J. Wu und A. Ignatiev, electric - pulse - induced reversible resistance change effect in magnetoresistive films, Appl. Phys. Lett. 76, 2749 (2000) 


\section{Danksagungen}

Herrn Prof. Dr. Konrad Samwer danke ich für sein Engagement und seine persönliche Anteilnahme bei der Betreuung dieser Arbeit. Seine ständige Diskussionsbereitschaft, seine wertvollen Anregungen und seine wohlwollende Unterstützung haben entscheidend zum Gelingen dieser Arbeit beigetragen.

Besonders herzlich danke ich Herrn Dr. Bernd Damaschke für seine uneingeschränkte Unterstützung, für viele anregende Diskussionen und wertvolle Ratschläge und für die freundschaftliche Betreuung dieser Arbeit.

Herrn Christoph Streng und Herrn Dr. Cay-Uwe Pinnow möchte ich für die freundschaftliche Zusammenarbeit und für die stete Hilfsbereitschaft bedanken.

Herrn Dr. Vasily Mosneaga und Herrn Dr. Yuansu Luo danke ich für die gemeinsamen Arbeiten und freundschaftlicher Atmosphäre.

Einen ganz besonderen Dank richte ich an Herrn Prof. Dr. Klaus Winzer und Herrn Prof. Dr. Wolfgang Felsch für die vielen angeregten Diskussionen.

Allen Mitarbeitern des I. Physikalischen Instituts, stellvertretend Frau Sigrun Köster, danke ich für die angenehme Arbeitsatmosphäre und die schöne Zeit.

Frau Gerda Brocks gilt mein Dank, stets freundlich und engagiert vieles für meine Arbeit im Sonderforschungsbereich 602 in die Wege geleitet zu haben.

Mein Dank gilt auch Herrn Carsten Mahn für seinen technischen Sachverstand, sowie Herrn Hartmut Eichenberg, Herrn Elmar Gatzemeier und Herrn Axel Bruchmann für so manches fachliches Gespräch.

Nicht zuletzt danke ich meinen Eltern und meiner Schwester. Sie haben mich während dieser Zeit stets aus vollen Kräften unterstützt und mich liebevoll begleitet. 


\section{Lebenslauf}

Name: $\quad$ Thomas Becker

Geburtsdatum: $\quad 27.10 .1974$

Geburtsort: $\quad$ Bad Karlshafen

1981 - 1985: $\quad$ Sieburgschule - Bad Karlshafen

1985 - 1991: $\quad$ Gesamtschule Bad Karlshafen

1991 - 1994: $\quad$ Albert - Schweizer Oberstufengymnasium Hofgeismar

1994: $\quad$ Abitur

1994 - 2000: $\quad$ Studium der Physik an der Georg - August - Universität Göttingen

1997: $\quad$ Vordiplom

2000: $\quad$ Hauptdiplom

Thema: Festkörperreaktionen an amorphen $-\mathrm{Al}_{30} \mathrm{Zr}_{70} / \mathrm{Ni}$ und amorphen $\mathrm{Ni}_{50} \mathrm{Zr}_{50} / \mathrm{Ni}$ Doppelschichten

2000 -2004: Wissenschaftlicher Mitarbeiter im Sonderforschungsbereich 345 und 602 der Georg - August - Universität Göttingen

Anfertigen der vorliegenden Dissertation am I. Physikalischen Institut der Georg - August - Universität Göttingen bei Prof. Dr. K. Samwer 